Supplemental material for:

\title{
Unified Approach to the Spiro(pyrrolidinyl-oxindole) and Hexahydropyrrolo[2,3-b]indole Alkaloids: Total Syntheses of Pseudophrynamines 270 and 272A
}

\author{
Subhadip De, Mrinal Kanti Das, Subhajit Bhunia, and Alakesh Bisai* \\ Department of Chemistry, Indian Institute of Science Education and Research \\ Bhopal, Bhopal Bypass Road, Bhopal - 462 066, MP, INDIA. \\ E-Mail: alakesh@,iiserb.ac.in
}

Table of Contents

$\begin{array}{ll}\text { Materials and Methods } & \mathrm{S} 2 \\ \text { General Procedure for starting materials preparation } & \mathrm{S} 3-\mathrm{S} 28 \\ \begin{array}{l}\text { General Procedure for organocatalytic Aldol Reaction } \\ \text { Characterization of (6a-l) }\end{array} & \mathrm{S} 28 \\ \text { Formal Synthesis of (-)-physovenine (2e) } & \mathrm{S} 28-\mathrm{S} 35 \\ \text { Total Synthesis of (+)-coerulescine (1a) } & \mathrm{S} 35-\mathrm{S} 40 \\ \text { Total Synthesis of (+)-horsfiline (1b) } & \mathrm{S} 40-\mathrm{S} 42 \\ \text { Total Synthesis of (-)-deoxyeseroline (2c) } & \mathrm{S} 42-\mathrm{S} 44 \\ \text { Total Synthesis of (-)-esermethole (2a) } & \mathrm{S} 44-\mathrm{S} 48 \\ \text { First Total Syntheses of (-)-pseudophrynamines 272A (3d) and 270(3b) } & \text { S51-S56 } \\ \text { Spectral traces and HPLC data } & \text { S57-S160 }\end{array}$ 


\section{Materials and Methods}

Unless otherwise stated, reactions were performed in oven-dried glassware fitted with rubber septa under a nitrogen atmosphere and were stirred with Teflon-coated magnetic stirring bars. Liquid reagents and solvents were transferred via syringe using standard Schlenk techniques. Tetrahydrofuran (THF), diethyl ether $\left(\mathrm{Et}_{2} \mathrm{O}\right)$ was distilled over sodium/benzophenone ketyl. Acetonitrile, dichloromethane $\left(\mathrm{CH}_{2} \mathrm{Cl}_{2}\right)$, toluene, and benzene, toluene were distilled over calcium hydride. All other solvents and reagents were used as received unless otherwise noted. Reaction temperatures above $23{ }^{\circ} \mathrm{C}$ refer to oil bath temperature. Thin layer chromatography was performed using Merck Silicagel 60 F-254 precoated plates $(0.25 \mathrm{~mm})$ and visualized by UV irradiation, 2,4-DNP, anisaldehyde stain and other stains. Silicagel from Merck (particle size 100-200 mesh) was used for flash chromatography. Melting points were recorded on a digital melting point apparatus from Jyoti Scientific (AN ISO 9001:2000) and are uncorrected. ${ }^{1} \mathrm{H}$ and

${ }^{13} \mathrm{C}$ NMR spectra were recorded on Bruker $400,500 \mathrm{MHz}$ spectrometers with ${ }^{13} \mathrm{C}$ operating frequencies of $100,125 \mathrm{MHz}$, respectively. Chemical shifts $(\delta)$ are reported in ppm relative to the residual solvent signal $\left(\delta=7.26\right.$ for ${ }^{1} \mathrm{H}$ NMR and $\delta=77.0$ for ${ }^{13} \mathrm{C}$ NMR). Data for ${ }^{1} \mathrm{H}$ NMR spectra are reported as follows: chemical shift (multiplicity, coupling constants, number of hydrogen). Abbreviations are as follows: s (singlet), d (doublet), t (triplet), q (quartet), m (multiplet), br (broad). IR spectra were recorded on a FT-IR system (Spectrum BX) from PerkinElmer spectrometer and are reported in frequency of absorption $\left(\mathrm{cm}^{-1}\right)$. Only selected IR absorbencies are reported. Highresolution mass spectrometry (HRMS) data were recorded on MicrOTOF-Q-II mass spectrometer using methanol as solvent. Optical rotations were measured on an Autopol I automatic polarimeter, Rudolph research analytical. Enantiomeric excess was determined by chiral HPLC analysis performed on PerkinElmer Technologies HPLC system with Daicel Chiralpak AD-H, IB, IC and IC3 column. 


\section{Synthesis of $\pm(7 a)$ :}

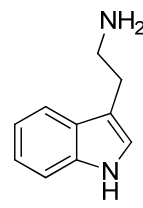

(16a)

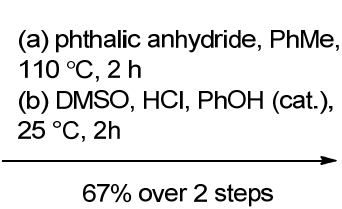

$67 \%$ over 2 steps

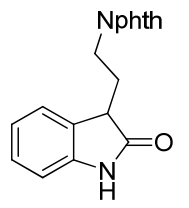

(7a)

Step 1: A mixture of tryptamine 16a $(5.0 \mathrm{~g}, 31.52 \mathrm{mmol} ; 1.0$ equiv) and phthalic anhydride (4.66 g, $31.52 \mathrm{mmol} ; 1.0$ equiv) in toluene $(50 \mathrm{~mL})$ was held at reflux for $2 \mathrm{~h}$ and then cooled to room temperature. The solvent was removed under vacuum and the resulting residue was dried under vacuum. The crude product was directly treated for next step without purification.

Step 2: In an oven-dried round-bottom flask, the crude phthalimido protected tryptamine (31.52 mmol, 1.0 equiv) was taken in acetic acid $(50 \mathrm{~mL})$. To this reaction mixture dimethyl sulphoxide (5.6 mL, 78.8 mmol; 2.5 equiv), $12(N) \mathrm{HCl}(14.4 \mathrm{~mL}, 156.6 \mathrm{mmol}$; 5.0 equiv) and phenol (148 mg, $1.576 \mathrm{mmol} ; 0.05$ equiv) was added respectively and it was stirred for $2 \mathrm{~h}$ at room temperature. Upon completion of the reactions, (TLC showed complete consumption of starting material) the reaction mixture was quenched with saturated $2(\mathrm{M}) \mathrm{NaOH}$ soln. and then diluted with $100 \mathrm{~mL}$ of EtOAc. The whole reaction mixture was taken in a separatory funnel and extracted with $30 \mathrm{~mL}$ of water. The organic filtrate was dried over $\mathrm{Na}_{2} \mathrm{SO}_{4}$ and concentrated in a rotary evaporator under vacuum. The crude products were purified by flash chromatography to afford 7a as white solid (6.46 g, 67\% overall yield in 2 steps), $\mathrm{R}_{f}=0.2$ (40\% EtOAc in hexane). ${ }^{1} \mathbf{H}$ NMR [400 MHz, DMSO : $\left.\mathrm{CDCl}_{3}(1: 4)\right] \delta: 10.0$ (brs, $\left.1 \mathrm{H}\right), 7.61-7.68(\mathrm{~m}, 4 \mathrm{H}), 7.18(\mathrm{~d}, J=7.4 \mathrm{~Hz}$, $1 \mathrm{H}), 6.97(\mathrm{t}, J=7.7 \mathrm{~Hz}, 1 \mathrm{H}), 6.74-6.77(\mathrm{~m}, 2 \mathrm{H}), 3.78-3.85(\mathrm{~m}, 1 \mathrm{H}), 3.64-3.70(\mathrm{~m}, 1 \mathrm{H})$, $3.36(\mathrm{t}, J=5.9 \mathrm{~Hz}, 1 \mathrm{H}), 2.29-2.38(\mathrm{~m}, 1 \mathrm{H}), 2.10-2.18(\mathrm{~m}, 1 \mathrm{H})$; IR (film) $v_{\max } 3307$, 2949, 2923, 2857, 1690, 1389, 1233, 1025, 738, $721 \mathrm{~cm}^{-1}$; MP $191{ }^{\circ} \mathrm{C}$.

\section{Procedure for the synthesis of $(7 \mathbf{b})$ :}




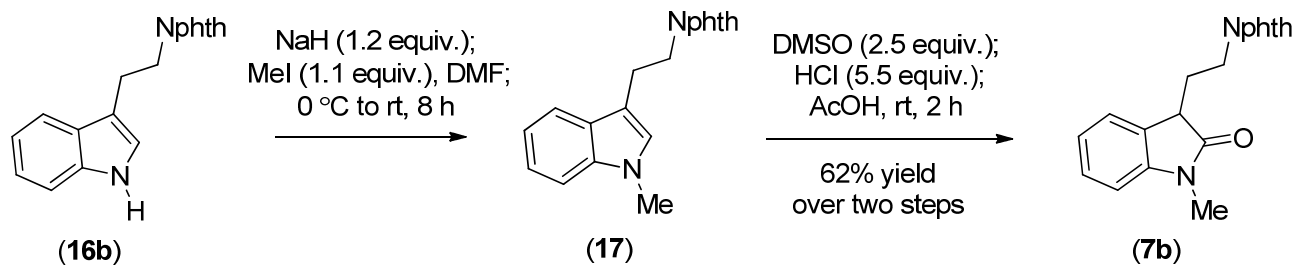

\section{Step 1:}

In an oven-dried round-bottom flask under argon atmosphere was charged with (2.0 g; $6.44 \mathrm{mmol} ; 1.0$ equiv) of compound $(\mathbf{1 6 b})$ in $\mathrm{DMF}(15 \mathrm{~mL})$ at $0{ }^{\circ} \mathrm{C}$. To this reaction mixture (309 mg; $7.73 \mathrm{mmol}, 1.2$ equiv.) of $\mathrm{NaH}(60 \%$ in mineral oil) was added pinch-wise. After 5 minutes of stirring methyl iodide $(0.42 \mathrm{~mL} ; 6.76 \mathrm{mmol}, 1.05$ equiv.) was added to the reaction mixture drop-wise. Upon completion of the reaction (monitoring by TLC), the reaction mixture was quenched with water $(40 \mathrm{~mL})$ and extracted with EtOAc $(50 \mathrm{~mL} X 2)$. The combined organic layer was dried over anhydrous sodium sulphate and concentrated under reduced pressure to afford desired $\mathrm{N}$ methylated product (17)

\section{Step 2:}

The crude compound (17) $(6.44 \mathrm{mmol} ; 1.0$ equiv) was taken in $\mathrm{AcOH}(30 \mathrm{~mL})$ at $25^{\circ} \mathrm{C}$. To this reaction mixture DMSO (1.6 mL; $16.1 \mathrm{mmol} ; 2.5$ equiv.) and Conc. $\mathrm{HCl}$ (3.0 mL; $35.42 \mathrm{mmol} ; 5.5$ equiv.) were added and stirring was continued for $2 \mathrm{~h}$ at $25^{\circ} \mathrm{C}$ (TLC showed complete consumption of starting material). Then the reaction mixture was diluted with $60 \mathrm{~mL}$ of EtOAc and $4(\mathrm{~N}) \mathrm{NaOH}$ solution was added drop-wise until $\mathrm{pH}$ of the mixture became 7 . The whole reaction mixture was taken in a separatory funnel and separated out the organic layer. The organic layer was dried over anhydrous $\mathrm{Na}_{2} \mathrm{SO}_{4}$ and concentrated in a rotary evaporator under reduced pressure. The crude products were purified by flash chromatography using EtOAc and hexane mixture (20-40\%) as eluent to afford the desired 2-oxindole derivative (7b) in $62 \%$ yield. 


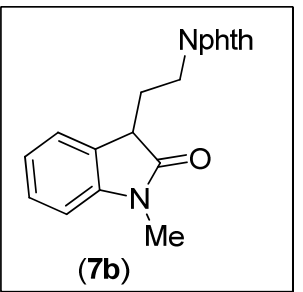

2-(2-(1-Methyl-2-oxoindolin-3-yl) ethyl) isoindoline-1,3-dione (7b): (62\% yield over two steps) of (7b) as a yellowish solid. $\mathrm{R}_{f}=0.23$ (30\% EtOAc in hexane); ${ }^{1} \mathbf{H}-\mathbf{N M R}$ (400 $\left.\mathrm{MHz}, \mathrm{CDCl}_{3}\right) \delta 7.55-7.62(\mathrm{~m}, 4 \mathrm{H}), 7.15(\mathrm{~d}, J=7.24 \mathrm{~Hz}, 1 \mathrm{H}), 6.98(\mathrm{t}, J=7.63 \mathrm{~Hz}, 1 \mathrm{H})$, $6.73(\mathrm{t}, J=7.51 \mathrm{~Hz}, 1 \mathrm{H}), 6.63(\mathrm{~d}, J=7.74 \mathrm{~Hz}, 1 \mathrm{H}), 3.78-3.85(\mathrm{~m}, 1 \mathrm{H}), 3.59-3.65(\mathrm{~m}$, 1H), $3.40(\mathrm{~m}, 1 \mathrm{H}), 3.07(\mathrm{~s}, 3 \mathrm{H}), 2.38-2.46(\mathrm{~m}, 1 \mathrm{H}), 2.22-2.28(\mathrm{~m}, 1 \mathrm{H}){ }^{13}$ C-NMR (100 $\left.\mathrm{MHz}, \mathrm{CDCl}_{3}\right) \delta 176.7,167.9,144.2,133.7,131.9,127.9,127.8,123.4,122.9,122.2$, 108.1, 43.4, 34.9, 27.9, 26.2; IR (film) $v_{\max }$ 2984, 1713, 1613, 1398, 1347, 1096, 1020, $752 \mathrm{~cm}^{-1}$; MP $129-133{ }^{\circ} \mathrm{C}$.

\section{Synthesis of (8a):}

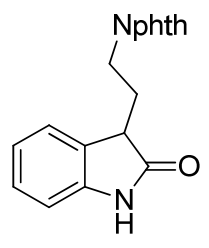

(7a)

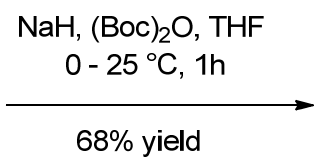

$68 \%$ yield

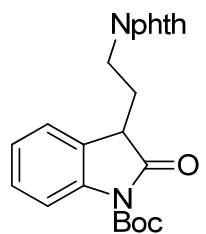

(8a)

In an oven-dried round-bottom flask, the compound 7a $(6.0 \mathrm{~g}, 19.6 \mathrm{mmol} ; 1.0$ equiv) was taken in dry THF $(50 \mathrm{~mL})$ under argon atmosphere at $0{ }^{\circ} \mathrm{C}$. To this reaction mixture $\mathrm{NaH}$ (862 mg, $21.56 \mathrm{mmol} ; 1.1$ equiv) was added portionwise and it was stirred for $5 \mathrm{~min}$. Boc-anhydride $(4.73 \mathrm{~mL}, 20.58 \mathrm{mmol}$; 1.05 equiv) was added to the reaction mixture dropwise $0{ }^{\circ} \mathrm{C}$ and it was stirred for $2 \mathrm{~h}$ at $\mathrm{rt}$. Upon completion of the reactions, (TLC showed complete consumption of starting material) the reaction mixture was quenched with ice-water and then diluted with $50 \mathrm{~mL}$ of EtOAc. The whole reaction mixture was taken in a separatory funnel and extracted with $50 \mathrm{~mL}$ of water. The organic filtrate was dried over $\mathrm{Na}_{2} \mathrm{SO}_{4}$ and concentrated in a rotary evaporator under vacuum. The crude products were purified by flash chromatography to afford $\mathbf{8 a}$ as colorless gel 
(5.4 g, 68\% yield). $\mathrm{R}_{f}=0.50$ (30\% EtOAc in hexane). ${ }^{1} \mathbf{H}$ NMR $\left(400 \mathrm{MHz}, \mathrm{CDCl}_{3}\right) \delta$ : 7.72-7.75 (m, 3H), 7.64-7.67 (m, 2H), $7.28(\mathrm{~d}, J=7.4 \mathrm{~Hz}, 2 \mathrm{H}), 7.14(\mathrm{t}, J=7.8 \mathrm{~Hz}, 1 \mathrm{H})$, $6.99(\mathrm{t}, J=7.4 \mathrm{~Hz}, 1 \mathrm{H}), 3.89-3.96(\mathrm{~m}, 1 \mathrm{H}), 3.75-3.82(\mathrm{~m}, 1 \mathrm{H}), 3.61(\mathrm{t}, J=5.9 \mathrm{~Hz}, 3 \mathrm{H})$, 2.41-2.48 (m, 1H), 2.30-2.39 (m, 1H), $\left.1.61(\mathrm{~s}, 9 \mathrm{H}) ;{ }^{13} \mathbf{C ~ N M R ~ ( 1 0 0 ~ M H z , ~} \mathrm{CDCl}_{3}\right) \delta$ 175.2, 168.0, 149.2, 140.1, 133.8, 132.0, 128.1, 126.8, 124.3, 123.4, 123.2, 115.1, 84.2, 44.0, 34.9, 28.9, 28.1; IR (film) $v_{\max } 2983,2936,1768,1715,1350,1253,1152,720 \mathrm{~cm}^{-}$ 1 .

\section{Synthesis and Characterization of (15c):}

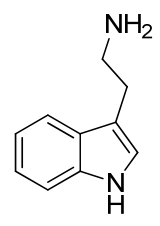

(16a)

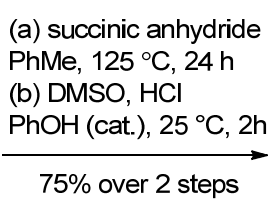

$75 \%$ over 2 steps

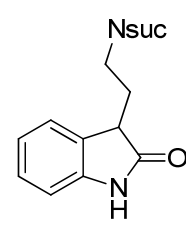

(16c)

\section{Step 1:}

A mixture of tryptamine 16a $(5.0 \mathrm{~g}, 31.52 \mathrm{mmol} ; 1.0$ equiv) and succinic anhydride anhydride (3.12 g, $31.52 \mathrm{mmol} ; 1.0$ equiv) in toluene : $\mathrm{CH}_{3} \mathrm{COOH}(1: 2)$ (90 $\mathrm{mL}$ ) was held at reflux (ca. $125^{\circ} \mathrm{C}$ ) for $24 \mathrm{~h}$ and then cooled toroom temperature. Hexanes $(50 \mathrm{~mL})$ were added to the reaction mixture and the product was removed by filtration and washed with hexanes $(50 \mathrm{~mL})$. The solvent was removed under vacuum and the resulting residue was dried under vacuum. The crude product was directly treated for next step without purification.

\section{Step 2:}

In an oven-dried round-bottom flask, the crude succimido protected tryptamine ( $1.0 \mathrm{~g}, 4.13 \mathrm{mmol} ; 1.0$ equiv) was taken in acetic acid $(15 \mathrm{~mL})$. To this reaction mixture dimethyl sulphoxide (734 $\mu \mathrm{L}, 10.33$ mmol; 2.5 equiv), $12(N) \mathrm{HCl}(1.7 \mathrm{~mL}, 20.66 \mathrm{mmol}$; 5.0 equiv) and phenol (19 mg, $0.207 \mathrm{mmol} ; 0.05$ equiv) was added respectively and it was stirred for $2 \mathrm{~h}$ at room temperature. Upon completion of the reactions, (TLC showed complete consumption of starting material) the reaction mixture was quenched with saturated $2(\mathrm{M}) \mathrm{NaOH}$ soln. and then diluted with $50 \mathrm{~mL}$ of EtOAc. The whole reaction mixture was taken in a separatory funnel and extracted with $20 \mathrm{~mL}$ of water. The organic 
filtrate was dried over $\mathrm{Na}_{2} \mathrm{SO}_{4}$ and concentrated in a rotary evaporator under vacuum. The crude products were purified by flash chromatography to afford 16c as yellow solid (799 mg, 75\% overall yield in 2 steps), $\mathrm{R}_{f}=0.20$ (75\% EtOAc in hexane). ${ }^{1} \mathbf{H}$ NMR [400 MHz, DMSO : $\left.\mathrm{CDCl}_{3}(1: 4)\right] \delta: 10.04$ (brs, 1H), 7.12 (d, $\left.J=7.1 \mathrm{~Hz}, 1 \mathrm{H}\right), 7.04$ (t, $J=7.6$ $\mathrm{Hz}, 1 \mathrm{H}), 6.84$ (t, $J=7.4 \mathrm{~Hz}, 1 \mathrm{H}), 6.74$ (d, $J=7.6 \mathrm{~Hz}, 1 \mathrm{H}), 3.57-3.62$ (m, 1H), 3.36-3.43 (m, 1H), $3.30(\mathrm{t}, J=5.7 \mathrm{~Hz}, 1 \mathrm{H}), 2.33-2.44(\mathrm{~m}, 4 \mathrm{H}), 2.19-2.26(\mathrm{~m}, 1 \mathrm{H}), 1.97-2.05(\mathrm{~m}$, $1 \mathrm{H}) ;{ }^{13} \mathrm{C}$ NMR $\left[100 \mathrm{MHz}, \mathrm{DMSO}: \mathrm{CDCl}_{3}(1: 4)\right] \delta 178.8,177.2,142.8,128.8,127.9$, 123.6, 121.6, 109.9, 43.9, 35.8, 28.0, 27.0; IR (film) $v_{\max }$ 3399, 1651, 1402, 1152, 751, $667 \mathrm{~cm}^{-1}$; MP $152-154{ }^{\circ} \mathrm{C}$.

\section{Synthesis and Characterization of (8b):}

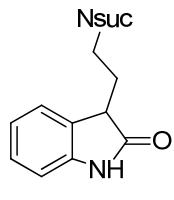

$(16 c)$

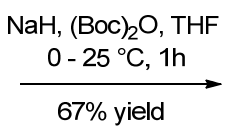

$67 \%$ yield

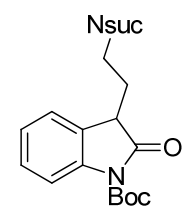

(8b)

In an oven-dried round-bottom flask, the compound 16c (522 mg, $2.02 \mathrm{mmol} ; 1.0$ equiv) was taken in dry THF $(15 \mathrm{~mL})$ under argon atmosphere at $0{ }^{\circ} \mathrm{C}$. To this reaction mixture $\mathrm{NaH}$ (89 mg, $2.26 \mathrm{mmol}$; 1.1 equiv) was added portionwise and it was stirred for $5 \mathrm{~min}$. Boc-anhydride (488 $\mu \mathrm{L} \mathrm{mL}, 2.124 \mathrm{mmol} ; 1.05$ equiv) was added to the reaction mixture dropwise $0{ }^{\circ} \mathrm{C}$ and it was stirred for $2 \mathrm{~h}$ at $\mathrm{rt}$. Upon completion of the reactions, (TLC showed complete consumption of starting material) the reaction mixture was quenched with ice-water and then diluted with $25 \mathrm{~mL}$ of EtOAc. The whole reaction mixture was taken in a separatory funnel and extracted with $30 \mathrm{~mL}$ of water. The organic filtrate was dried over $\mathrm{Na}_{2} \mathrm{SO}_{4}$ and concentrated in a rotary evaporator under vacuum. The crude products were purified by flash chromatography to afford $\mathbf{8 b}$ as colorless gel (485 $\mathrm{mg}$, $67 \%$ yield), $\mathrm{R}_{f}=0.3\left(30 \%\right.$ EtOAc in hexane). ${ }^{1} \mathbf{H}$ NMR $\left(400 \mathrm{MHz}, \mathrm{CDCl}_{3}\right) \delta: 7.78(\mathrm{~d}, J=$ $8.1 \mathrm{~Hz}, 1 \mathrm{H}), 7.37(\mathrm{~d}, J=7.4 \mathrm{~Hz}, 1 \mathrm{H}), 7.33(\mathrm{t}, J=7.9 \mathrm{~Hz}, 1 \mathrm{H}), 7.16(\mathrm{t}, J=7.5 \mathrm{~Hz}, 1 \mathrm{H})$, 3.68-3.75 (m, 1H), 3.50-3.55 (m, 1H), 2.53 (brs, 4H), 2.31-2.38 (m, 1H), 2.16-2.22 (m, 1H), 2.0 (brs, 1H), 1.60 (s, 9H); ${ }^{13} \mathrm{C}$ NMR (100 MHz, $\left.\mathrm{CDCl}_{3}\right) \delta 177.6,176.2,148.8$, 
139.4, 130.2, 128.6, 125.0, 123.7, 115.5, 84.8, 75.6, 35.3, 33.6, 29.7, 28.1; IR (film) $v_{\max }$ $2985,2932,1707,1252,1151,1099,772 \mathrm{~cm}^{-1}$.

\section{General Procedure for Synthesis of $N$-methyl- $N$-protected tryptamines (15e-g):}

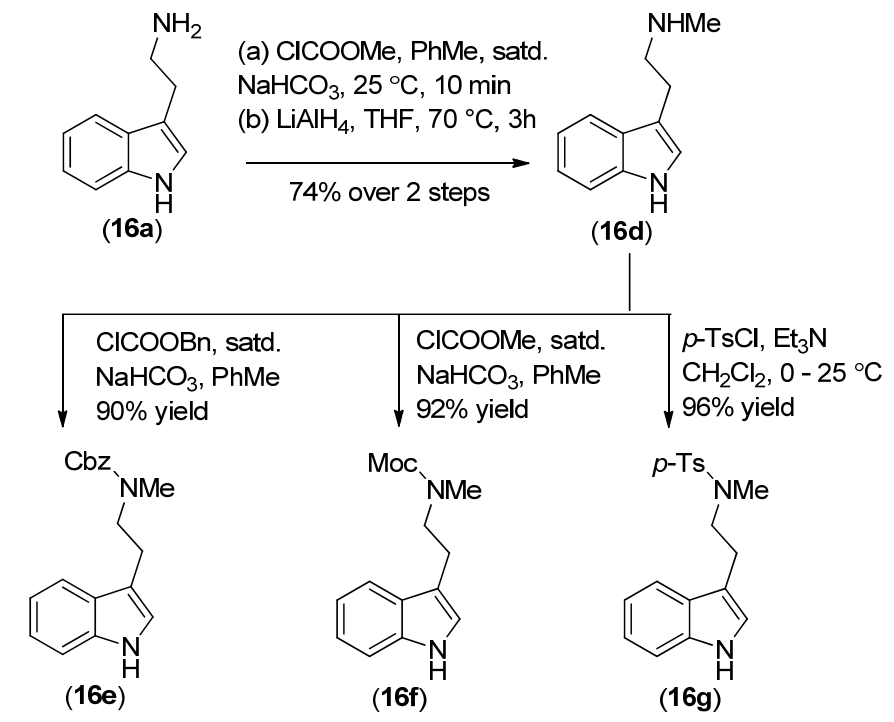

\section{Procedure for synthesis of $N$-methyl tryptamine (16d):}

\section{Step 1:}

A round-bottom flask was charged with tryptamine $\mathbf{1 6 a}(5.0 \mathrm{gm}, 31.21 \mathrm{mmol} ; 1.0$ equiv) in toluene : $\mathrm{NaHCO}_{3}(1: 1)(60 \mathrm{~mL})$. To this reaction mixture methyl chloroformate (2.66 mL, $34.32 \mathrm{mmol}$; 1.1 equiv) was added dropwise and it was stirred for $30 \mathrm{~min}$ at room temperature. Upon completion of the reaction (monitoring by TLC), it was diluted by $100 \mathrm{~mL}$ EtOAc. The whole reaction mixture was taken in a separatory funnel and extracted with $75 \mathrm{~mL}$ of water. The organic filtrate was dried over anhydrous $\mathrm{Na}_{2} \mathrm{SO}_{4}$ and concentrated in a rotary evaporator under vacuum. The crude material was directly treated for next step without isolation.

\section{Step 2:}

The crude material (31.21 mmol; 1.0 equiv. as prepared earlier) was taken in dry THF $(100 \mathrm{~mL})$ under argon atmosphere and the reaction vessel was cooled to $0{ }^{\circ} \mathrm{C}$. To this reaction mixture $\mathrm{LiAlH}_{4}(2.37 \mathrm{~g}, 62.42 \mathrm{mmol} ; 2.0$ equiv. $)$ was added portion-wise. After stirring at $0{ }^{\circ} \mathrm{C}$ for 5 minutes, the reaction mixture was warmed to $23{ }^{\circ} \mathrm{C}$ and stirring 
continued for another 10 minutes. Then, the reaction mixture was refluxed on an oil-bath maintaining the temperature to $80{ }^{\circ} \mathrm{C}$ and stirring continued for $6 \mathrm{~h}$. Upon completion of the reaction (monitoring by TLC), it was cooled to room temperature and then to $0{ }^{\circ} \mathrm{C}$ and quenched with EtOAc, basified with $4(\mathrm{~N}) \mathrm{NaOH}$ solution and extracted with EtOAc $(2 \mathrm{X}$ $60 \mathrm{~mL})$. The combined organic extracts were washed with saturated aq. $\mathrm{NaCl}(50 \mathrm{~mL})$, dried over anhydrous $\mathrm{Na}_{2} \mathrm{SO}_{4}$, and concentrated under reduced pressure. The crude products $16 \mathbf{d}$ were directly treated for next steps without purification.

\section{Procedure for synthesis of (16e):}

A round-bottom flask was charged with $N$-methyl tryptamine $(10.4 \mathrm{mmol} ; 1.0$ equiv) in toluene : $\mathrm{NaHCO}_{3}(1: 1)(40 \mathrm{~mL})$. To this reaction mixture benzyl chloroformate (3.9 mmol; 1.1 equiv) was added dropwise and it was stirred for $30 \mathrm{~min}$ at room temperature. Upon completion of the reaction (monitoring by TLC), it was diluted by 50 $\mathrm{mL}$ EtOAc. The whole reaction mixture was taken in a separatory funnel and extracted with $30 \mathrm{~mL}$ of water. The organic filtrate was dried over anhydrous $\mathrm{Na}_{2} \mathrm{SO}_{4}$ and concentrated in a rotary evaporator under vacuum. The crude products were purified by flash chromatography to afford pure $\mathbf{1 6 e}$.

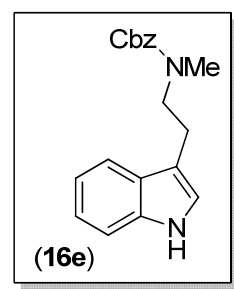

Benzyl (2-(1H-indol-3-yl)ethyl)(methyl)carbamate (16e): $2.15 \mathrm{~g}$ (67\% yields) as colorless gel. $\mathrm{R}_{f}=0.40\left(30 \%\right.$ EtOAc in hexane). ${ }^{1} \mathbf{H}$ NMR $\left(400 \mathrm{MHz}, \mathrm{CDCl}_{3}\right)$ (rotameric mixture) $\delta: 8.1$ (brs, 1H), 7.47-7.64 (m, 1H), 6.92-7.34 (m, 9H), 5.09-5.15 (m, 2H), 3.58 (brs, 2H), 3.0 (brs, 2H), 2.92 (s, 3H); ${ }^{13} \mathbf{C ~ N M R ~ ( 1 0 0 ~ M H z , ~} \mathrm{CDCl}_{3}$ ) (rotameric mixture) $\delta: 156.4,136.9,136.3,128.5,128.0,127.4,122.0,121.98,119.4,118.7,112.9,111.2$, 67.1, 67.0, 49.8, 49.7, 34.9, 34.8, 24.1, 24.0; IR (film) $v_{\max } 3386,2940,1672,1440$, $1207,1138,745,700 \mathrm{~cm}^{-1}$.

\section{Procedure for synthesis of (16f):}


An oven-dried round-bottom flask was charged with $N$-methyl tryptamine (10.4 mmol; 1.0 equiv.) in $\mathrm{CH}_{2} \mathrm{Cl}_{2}(20 \mathrm{~mL})$ under argon atmosphere and the reaction vessel was cooled to $0{ }^{\circ} \mathrm{C}$. To this reaction mixture $\mathrm{Et}_{3} \mathrm{~N}$ (1.88 $\mathrm{mL}, 13.52 \mathrm{mmol} ; 1.3$ equiv), methyl chloroformate ( $805 \mu \mathrm{L}, 10.4 \mathrm{mmol} ; 1.0$ equiv) and DMAP (127 mg, $1.04 \mathrm{mmol}$; 0.1 equiv) was added and it was stirred for overnight. Upon completion of the reactions, (TLC showed complete consumption of starting material) the reaction mixture was quenched with water $(30 \mathrm{~mL})$ and diluted with another $30 \mathrm{~mL}$ of $\mathrm{CH}_{2} \mathrm{Cl}_{2}$. The whole reaction mixture was taken in a separatory funnel and extracted the organic layer. The organic filtrate was dried over anhydrous $\mathrm{Na}_{2} \mathrm{SO}_{4}$ and concentrated in a rotary evaporator under vacuum. The crude products were purified by flash chromatography to afford pure 16f.

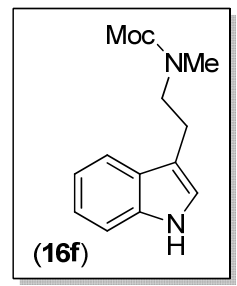

Methyl (2-(1H-indol-3-yl)ethyl)(methyl)carbamate (16f): $1.64 \mathrm{~g}$ (68\% yields) as yellow solid. $\mathrm{R}_{f}=0.30\left(30 \%\right.$ EtOAc in hexane). ${ }^{1} \mathbf{H}$ NMR $\left(400 \mathrm{MHz}, \mathrm{CDCl}_{3}\right)$ (rotameric mixture) $\delta: 8.24(\mathrm{~s}, 1 \mathrm{H}), 7.62-7.64(\mathrm{~m}, 1 \mathrm{H}), 7.34(\mathrm{~d}, J=8.0 \mathrm{~Hz}, 1 \mathrm{H}), 7.19(\mathrm{t}, J=7.1 \mathrm{~Hz}$, 1H), $7.12(\mathrm{t}, J=7.4 \mathrm{~Hz}, 1 \mathrm{H}), 6.98(\mathrm{~d}, J=9.1 \mathrm{~Hz}, 1 \mathrm{H}), 3.53-3.72(\mathrm{~m}, 5 \mathrm{H}), 2.99$ (brs, 2H), 2.87-2.90 (m, 3H); ${ }^{13} \mathrm{C}$ NMR $\left(100 \mathrm{MHz}, \mathrm{CDCl}_{3}\right)$ (rotameric mixture) $\delta: 157.09,157.06$, $136.4,127.5,127.4,122.0,119.3,118.7,118.6,113.0,112.9,111.2,52.5,50.1,49.4$, 35.0, 34.5, 24.2, 23.6; IR (film) $v_{\max } 3435,2949,1655,1209,961,743 \mathrm{~cm}^{-1}$; MP 66-68 ${ }^{\circ} \mathrm{C}$.

\section{Procedure for synthesis of (16g):}

An oven-dried round-bottom flask was charged with $N$-methyl tryptamine (10.4 mmol; 1.0 equiv. $)$ in $\mathrm{CH}_{2} \mathrm{Cl}_{2}(20 \mathrm{~mL})$ under argon atmosphere and the reaction vessel was cooled to $0{ }^{\circ} \mathrm{C}$. To this reaction mixture $\mathrm{Et}_{3} \mathrm{~N}(1.88 \mathrm{~mL}, 13.52 \mathrm{mmol} ; 1.3$ equiv), $p$ $\mathrm{TsCl}$ (1.98 gm, $10.4 \mathrm{mmol} ; 1.0$ equiv) and DMAP (127 mg, $1.04 \mathrm{mmol} ; 0.1$ equiv) was added and it was stirred for overnight. Upon completion of the reactions, (TLC showed 
complete consumption of starting material) the reaction mixture was quenched with water $(30 \mathrm{~mL})$ and diluted with another $30 \mathrm{~mL}$ of $\mathrm{CH}_{2} \mathrm{Cl}_{2}$. The whole reaction mixture was taken in a separatory funnel and extracted the organic layer. The organic filtrate was dried over anhydrous $\mathrm{Na}_{2} \mathrm{SO}_{4}$ and concentrated in a rotary evaporator under vacuum. The crude products were purified by flash chromatography to afford pure $\mathbf{1 6 g}$.

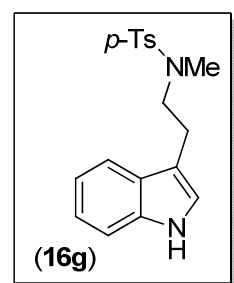

$N$-(2-(1H-indol-3-yl)ethyl)- $N$,4-dimethylbenzenesulfonamide $\quad$ (16g): $2.4 \quad \mathrm{~g} \quad$ (71\% yields) as yellow solid. $\mathrm{R}_{f}=0.60\left(30 \%\right.$ EtOAc in hexane). ${ }^{1} \mathbf{H}$ NMR $\left(400 \mathrm{MHz}, \mathrm{CDCl}_{3}\right)$ $\delta: 8.12$ (brs, 1H), $7.65(\mathrm{~d}, J=8.2 \mathrm{~Hz}, 2 \mathrm{H}), 7.55(\mathrm{~d}, J=7.8 \mathrm{~Hz}, 1 \mathrm{H}), 7.35(\mathrm{~d}, J=8.1 \mathrm{~Hz}$, 1H), $7.27(\mathrm{~d}, J=8.0 \mathrm{~Hz}, 1 \mathrm{H}), 7.16-7.2(\mathrm{~m}, 1 \mathrm{H}), 7.09-7.12(\mathrm{~m}, 1 \mathrm{H}), 7.04(\mathrm{~d}, J=2.0 \mathrm{~Hz}$, $1 \mathrm{H}), 3.33(\mathrm{t}, J=7.4 \mathrm{~Hz}, 2 \mathrm{H}), 3.02(\mathrm{t}, J=8.2 \mathrm{~Hz}, 2 \mathrm{H}), 2.79(\mathrm{~s}, 3 \mathrm{H}), 2.40(\mathrm{~s}, 3 \mathrm{H}) ;{ }^{13} \mathrm{C}$ NMR $\left(100 \mathrm{MHz}, \mathrm{CDCl}_{3}\right) \delta 143.3,136.3,134.8,129.7,127.4,127.3,122.3,122.1,119.4$, 118.5, 112.3, 111.3, 50.8, 35.1, 24.4, 21.5; IR (film) $v_{\max } 3415,2927,2862,1654,1339$, $1159,952,744 \mathrm{~cm}^{-1} ; \mathbf{M P} 80-82{ }^{\circ} \mathrm{C}$.

\section{General Procedure for Synthesis of (8c-e):}

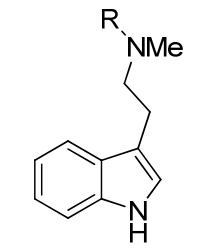

$\mathrm{R}=\mathrm{Cbz},(\mathbf{1 6 e})$

$\mathrm{R}=\mathrm{Moc},(\mathbf{1 6 f})$

$\mathrm{R}=p-\mathrm{Ts},(\mathbf{1 6 g})$ (a) $\mathrm{DMSO}, \mathrm{HCl}, \mathrm{PhOH}$ (cat.),

$\mathrm{AcOH}, \mathrm{rt}, 2 \mathrm{~h}$

(b) $\mathrm{NaH},(\mathrm{Boc})_{2} \mathrm{O}, \mathrm{THF}$,

0 - $25^{\circ} \mathrm{C}, 2 \mathrm{~h}$

upto $43 \%$ over 2 steps

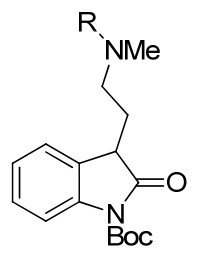

$\mathrm{R}=\mathrm{Cbz},(\mathbf{8 c})$

$\mathrm{R}=\mathrm{Moc},(\mathbf{8 d})$

$\mathrm{R}=p-\mathrm{Ts},(\mathbf{8 e})$

\section{Step 1:}

In an oven-dried round-bottom flask, the protected $N$-methyl tryptamines 16e-g (4.0 mmol; 1.0 equiv) was taken in acetic acid $(12 \mathrm{~mL})$. To this reaction mixture dimethyl 
sulphoxide ( $710 \mu \mathrm{L}, 10.0 \mathrm{mmol} ; 2.5$ equiv), $12(N) \mathrm{HCl}(1.67 \mathrm{~mL}, 20.0 \mathrm{mmol} ; 5.0$ equiv) and phenol (19 mg, $0.2 \mathrm{mmol} ; 0.05$ equiv) was added respectively and it was stirred for 2 $\mathrm{h}$ at room temperature. Upon completion of the reactions, (TLC showed complete consumption of starting material) the reaction mixture was quenched with saturated $2(\mathrm{M})$ $\mathrm{NaOH}$ soln. and then diluted with $50 \mathrm{~mL}$ of EtOAc. The whole reaction mixture was taken in a separatory funnel and extracted with $20 \mathrm{~mL}$ of water. The organic filtrate was dried over $\mathrm{Na}_{2} \mathrm{SO}_{4}$ and concentrated in a rotary evaporator under vacuum. The crude products were purified by flash chromatography to afford 18a-c.

\section{Step 2:}

In an oven-dried round-bottom flask, the compound 18a-c (1.0 mmol; 1.0 equiv) was taken in dry THF $(10 \mathrm{~mL})$ under argon atmosphere at $0{ }^{\circ} \mathrm{C}$. To this reaction mixture $\mathrm{NaH}$ (49 mg, $1.1 \mathrm{mmol}$; 1.1 equiv) was added portionwise and it was stirred for $5 \mathrm{~min}$. Boc-anhydride (241 $\mu \mathrm{L}, 1.05 \mathrm{mmol} ; 1.05$ equiv) was added to the reaction mixture dropwise at $0{ }^{\circ} \mathrm{C}$ and it was stirred for $2 \mathrm{~h}$ at room temperature. Upon completion of the reactions, (TLC showed complete consumption of starting material) the reaction mixture was quenched with ice-water and then diluted with $20 \mathrm{~mL}$ of EtOAc. The whole reaction mixture was taken in a separatory funnel and extracted with $15 \mathrm{~mL}$ of water. The organic filtrate was dried over $\mathrm{Na}_{2} \mathrm{SO}_{4}$ and concentrated in a rotary evaporator under vacuum. The crude products were purified by flash chromatography to afford $\mathbf{8 c - e}$.

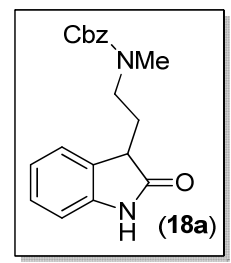

Benzyl methyl(2-(2-oxoindolin-3-yl)ethyl)carbamate (18a): Yellow gel. $\mathrm{R}_{f}=0.25$ (40\% EtOAc in hexane). ${ }^{1} \mathbf{H}$ NMR $\left(400 \mathrm{MHz}, \mathrm{CDCl}_{3}\right.$ ) (rotameric mixture) $\delta: 8.93$ (brs, 0.51H), 8.87 (brs, 0.49H), 7.24-7.31 (m, 5.59H), 7.17 (t, $J=7.6 \mathrm{~Hz}, 1 \mathrm{H}), 6.85-7.03$ (m, 2.41H), 5.01-5.11 (m, 2H), 4.61 (brs, $1 \mathrm{H}), 3.43(\mathrm{~m}, 1 \mathrm{H}), 3.28-3.35(\mathrm{~m}, 1 \mathrm{H}), 2.90(\mathrm{~s}, 3 \mathrm{H})$, 2.16 (brs, $2 \mathrm{H}) ;{ }^{13} \mathrm{C}$ NMR $\left(100 \mathrm{MHz}, \mathrm{CDCl}_{3}\right.$ ) (rotameric mixture) $\delta: 180.5,180.3,156.33$, $156.27,141.9,141.8,136.8,136.7,129.3,128.9,128.5,128.0,127.8,124.2,123.9,122.4$, 
110.0, 67.2, 67.1, 46.3, 45.7, 43.8, 34.8, 34.1, 28.4, 28.2; IR (film) $v_{\max } 3263,2930$, $1703,1624,1471,1406,1229,1149,753,700 \mathrm{~cm}^{-1}$.

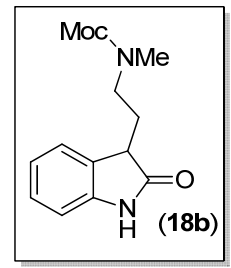

Methyl methyl(2-(2-oxoindolin-3-yl)ethyl)carbamate (18b): Yellow gel. $\mathrm{R}_{f}=0.18$ (50\% EtOAc in hexane). ${ }^{1} \mathbf{H}$ NMR $\left(400 \mathrm{MHz}, \mathrm{CDCl}_{3}\right)$ (rotameric mixture) $\delta: 9.06$ (brs, 0.5H), 8.97 (brs, 0.5H), 7.18-7.34 (m, 2H), $7.01(\mathrm{t}, J=7.2 \mathrm{~Hz}, 1 \mathrm{H}), 6.89(\mathrm{~m}, 1 \mathrm{H}), 3.62$ $(\mathrm{s}, 1.5 \mathrm{H}), 3.59(\mathrm{~s}, 1.5 \mathrm{H}), 3.44(\mathrm{t}, J=6.0 \mathrm{~Hz}, 1 \mathrm{H}), 3.34(\mathrm{~m}, 2 \mathrm{H}), 2.86(\mathrm{~s}, 3 \mathrm{H}), 2.18-2.20$ $(\mathrm{m}, 2 \mathrm{H}) ;{ }^{13} \mathrm{C}$ NMR $\left(100 \mathrm{MHz}, \mathrm{CDCl}_{3}\right) \delta 180.3,178.0,174.9,157.0,141.8,141.5,129.2$, $128.9,128.1,128.0,124.3,123.9,122.5,122.4,109.9,109.8,53.4,52.6,46.2$, 45.6, 43.7, 34.6, 34.1, 29.7, 29.6, 28.3; IR (film) $v_{\max }$ 3455, 2958, 2930, 1685, 1230, 1202, 1141, $754 \mathrm{~cm}^{-1}$.

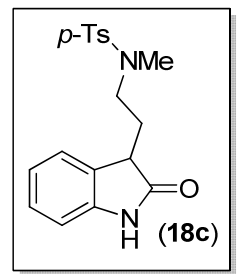

$N$,4-Dimethyl- $N$-(2-(2-oxoindolin-3-yl)ethyl)benzenesulfonamide (18c): Yellow solid. $\mathrm{R}_{f}=0.30$ (50\% EtOAc in hexane). ${ }^{1} \mathbf{H}$ NMR (400 MHz, $\left.\mathrm{CDCl}_{3}\right) \delta: 8.51$ (brs, $\left.1 \mathrm{H}\right), 7.62$ $(\mathrm{d}, J=8.2 \mathrm{~Hz}, 1 \mathrm{H}), 7.35(\mathrm{~d}, J=7.4 \mathrm{~Hz}, 1 \mathrm{H}), 7.19-7.29(\mathrm{~m}, 3 \mathrm{H}), 7.04(\mathrm{t}, J=7.5 \mathrm{~Hz}, 1 \mathrm{H})$, $6.87(\mathrm{~d}, J=7.8 \mathrm{~Hz}, 1 \mathrm{H}), 3.54(\mathrm{t}, J=6.4 \mathrm{~Hz}, 1 \mathrm{H}), 3.28-3.35(\mathrm{~m}, 1 \mathrm{H}), 3.04-3.11(\mathrm{~m}, 1 \mathrm{H})$, $2.73(\mathrm{~s}, 3 \mathrm{H}), 2.40(\mathrm{~s}, 3 \mathrm{H}), 2.08-2.21(\mathrm{~m}, 2 \mathrm{H}) ;{ }^{13} \mathbf{C}$ NMR $\left(100 \mathrm{MHz}, \mathrm{CDCl}_{3}\right) \delta 180.1$, $143.5,141.5,134.1,129.7,128.9,128.1,127.5,124.4,122.6,110.0,47.2,43.2,35.0$, 28.8, 21.5; IR (film) $v_{\max } 3349,2928,1703,1646,1470,1337,1160,970,815,753 \mathrm{~cm}^{-1}$; MP $80-82{ }^{\circ} \mathrm{C}$. 


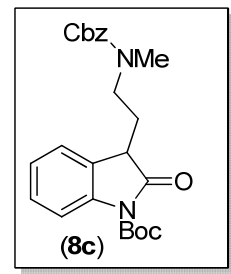

tert-Butyl 3-(2-(((benzyloxy)carbonyl)(methyl)amino)ethyl)-2-oxoindoline-1-

carboxylate (8c): $182 \mathrm{mg}$ (overall yield $43 \%$ in 2 steps) as colorless gel. $\mathrm{R}_{f}=0.70(50 \%$ EtOAc in hexane). ${ }^{1} \mathbf{H}$ NMR (400 $\mathrm{MHz}, \mathrm{CDCl}_{3}$ ) (rotameric mixture) $\delta: 7.77(\mathrm{~d}, J=8.2$ $\mathrm{Hz}, 1 \mathrm{H})$, 7.24-7.37 (m, 7H), 7.02-7.07 (m, 1H), 5.01-5.11 (m, 2H), 3.28-3.65 (m, 3H), $2.91(\mathrm{~s}, 3 \mathrm{H}), 2.18$ (brs, 2H), $1.62(\mathrm{~s}, 9 \mathrm{H}) ;{ }^{13} \mathrm{C} \mathrm{NMR}\left(100 \mathrm{MHz}, \mathrm{CDCl}_{3}\right.$ ) (rotameric mixture) $\delta: 172.6,169.6,156.4,149.2,140.0,139.9,136.8,136.76,136.7,136.67,128.5$, $128.2,128.1,128.0,127.9,127.79,127.7,124.5,124.48,124.4,123.92,123.89,123.44$, $114.9,84.38,84.37,67.2,67.1,46.2,45.6,43.5,36.6,34.8,34.2,29.1,29.0,28.1$; IR (film) $v_{\max } 2980,2935,1768,1730,1705,1465,1404,1350,1293,1252,1151,755 \mathrm{~cm}^{-1}$.

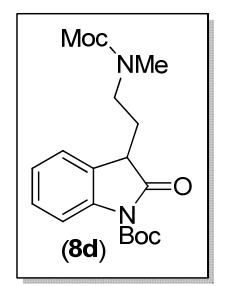

tert-Butyl carboxylate (8d): $143 \mathrm{mg}$ (overall yield $41 \%$ in 2 steps) as yellow gel. $\mathrm{R}_{f}=0.40(30 \%$ EtOAc in hexane). ${ }^{1} \mathbf{H}$ NMR (400 $\mathrm{MHz}, \mathrm{CDCl}_{3}$ ) (rotameric mixture) $\delta: 7.76$ (brs, $1 \mathrm{H}$ ), 7.23-7.34 (m, 2H), $7.11(\mathrm{t}, J=7.5 \mathrm{~Hz}, 1 \mathrm{H}), 3.48-3.63$ (m, 5H), 3.29 (brs, 1H), 2.82 (s, 3H), 2.41 (brs, 2H), 1.59 (s, 9H); ${ }^{13} \mathbf{C}$ NMR (100 MHz, $\mathrm{CDCl}_{3}$ ) (rotameric mixture) $\delta$ : $175.9,175.5,157.0,156.7,149.1,149.0,140.0,139.9,130.4,128.2,127.3,127.1,124.4$, 123.83, 123.78, 123.4, 115.2, 114.9, 85.4, 84.3, 52.6, 46.0, 45.4, 43.5, 34.1, 29.7, 28.9, 28.1, 28.0; IR (film) $v_{\max } 2980,2934,1768,1732,1465,1349,1292,1251,1151,1096$, $770 \mathrm{~cm}^{-1}$. 


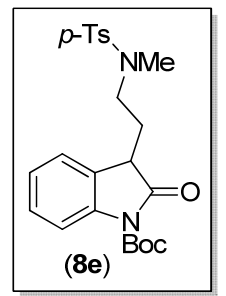

tert-Butyl
3-(2-((N,4-dimethylphenyl)sulfonamido)ethyl)-2-oxoindoline-1-

carboxylate (8e): $173 \mathrm{mg}$ (overall yield $39 \%$ in 2 steps) as colorless gel. $\mathrm{R}_{f}=0.40(20 \%$ EtOAc in hexane). ${ }^{1} \mathbf{H}$ NMR $\left(400 \mathrm{MHz}, \mathrm{CDCl}_{3}\right) \delta: 7.74(\mathrm{~d}, J=8.1 \mathrm{~Hz}, 1 \mathrm{H}), 7.56(\mathrm{~d}, J=$ $8.2 \mathrm{~Hz}, 2 \mathrm{H}), 7.30(\mathrm{~d}, J=7.4 \mathrm{~Hz}, 1 \mathrm{H}), 7.19-7.24(\mathrm{~m}, 3 \mathrm{H}), 7.12(\mathrm{t}, J=7.4 \mathrm{~Hz}, 1 \mathrm{H}), 3.6(\mathrm{t}$, $J=6.2 \mathrm{~Hz}, 1 \mathrm{H}), 3.17-3.24(\mathrm{~m}, 1 \mathrm{H}), 3.01-3.07(\mathrm{~m}, 1 \mathrm{H}), 2.67$ (s, 3H), $2.34(\mathrm{~s}, 3 \mathrm{H}), 2.11(\mathrm{q}$, $J=6.8 \mathrm{~Hz}, 2 \mathrm{H}), 1.57(\mathrm{~s}, 9 \mathrm{H}) ;{ }^{13} \mathrm{C}$ NMR $\left(100 \mathrm{MHz}, \mathrm{CDCl}_{3}\right) \delta 176.0,149.1,143.5,140.1$, $134.0,129.7,128.3,127.5,127.1,124.5,123.9,115.1,84.4,47.2,43.1,35.2,29.73$, 29.69, 28.1, 21.5; IR (film) $v_{\max }$ 2980, 2930, 1787, 1734, 1344, 1294, 1251, 1162, 1091, $738 \mathrm{~cm}^{-1}$.

\section{General Procedure for Synthesis of $N$-methyl- $N$-protected tryptamines (16i-k):}
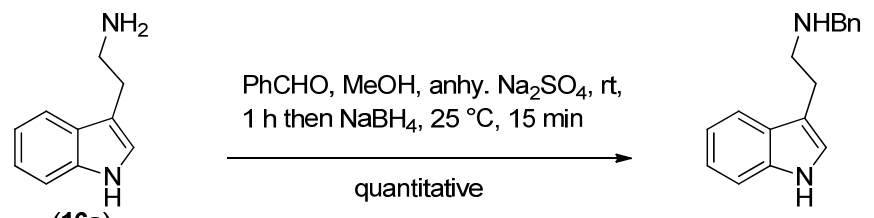

(16a)
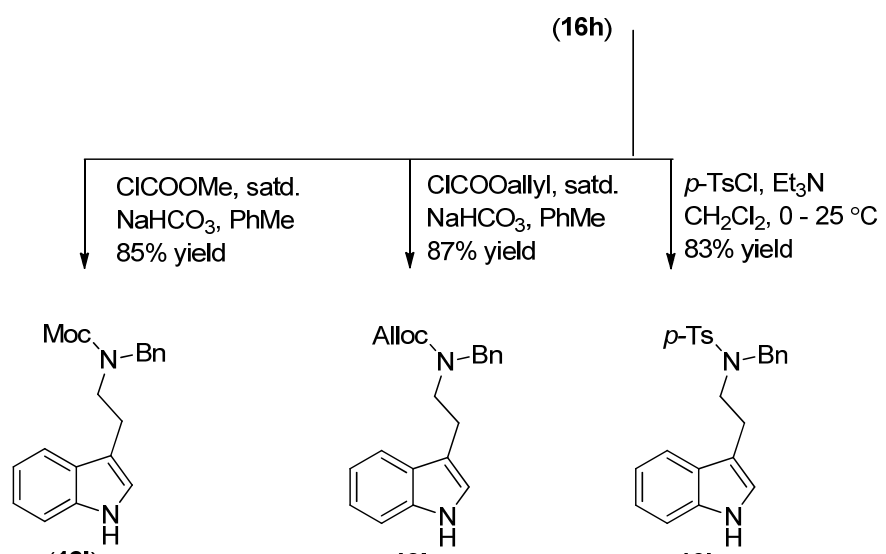

(16j)

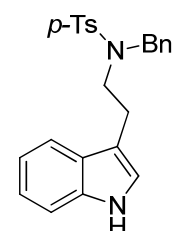

(16k)

In an oven-dried round-bottom flask, tryptamine 16a $(2.88 \mathrm{gm}, 18.0 \mathrm{mmol} ; 1.0$ equiv), benzaldehyde (1.83 mL, $18.0 \mathrm{mmol} ; 1.0$ equiv) and anhydrous $\mathrm{Na}_{2} \mathrm{SO}_{4}$ were taken 
in dry methanol $(20 \mathrm{~mL})$ under argon atmosphere at room temperature. The reaction mixture was stirred for $1 \mathrm{~h}$ at room temperature, $\mathrm{NaBH}_{4}(1.37 \mathrm{gm}, 36.0 \mathrm{mmol} ; 2.0$ equiv) was added to the reaction mixture portionwise at $0{ }^{\circ} \mathrm{C}$ and it was stirred for $30 \mathrm{~min}$ at $\mathrm{rt}$. Upon completion of the reactions, (TLC showed complete consumption of starting material) the reaction mixture was quenched with water and then diluted with $50 \mathrm{~mL}$ of $\mathrm{CH}_{2} \mathrm{Cl}_{2}$. The whole reaction mixture was taken in a separatory funnel and extracted with $20 \mathrm{~mL}$ of water. The organic filtrate was dried over $\mathrm{Na}_{2} \mathrm{SO}_{4}$ and concentrated in a rotary evaporator under vacuum. The crude product $\mathbf{1 6 h}$ was treated for next step without purification.

\section{Procedure for synthesis of (16i):}

An oven-dried round-bottom flask was charged with $N$-benzyl tryptamine $\mathbf{1 6 h}$ (6.0 mmol; 1.0 equiv.) in $\mathrm{CH}_{2} \mathrm{Cl}_{2}(20 \mathrm{~mL})$ under argon atmosphere and the reaction vessel was cooled to $0{ }^{\circ} \mathrm{C}$. To this reaction mixture $\mathrm{Et}_{3} \mathrm{~N}(1.25 \mathrm{~mL}, 9.0 \mathrm{mmol} ; 1.5$ equiv), methyl chloroformate ( $465 \mu \mathrm{L}, 6.0 \mathrm{mmol} ; 1.0$ equiv) and DMAP (73 mg, $0.6 \mathrm{mmol} ; 0.1$ equiv) was added and it was stirred for overnight. Upon completion of the reactions, (TLC showed complete consumption of starting material) the reaction mixture was quenched with water $(30 \mathrm{~mL})$ and diluted with another $30 \mathrm{~mL}$ of $\mathrm{CH}_{2} \mathrm{Cl}_{2}$. The whole reaction mixture was taken in a separatory funnel and extracted the organic layer. The organic filtrate was dried over anhydrous $\mathrm{Na}_{2} \mathrm{SO}_{4}$ and concentrated in a rotary evaporator under vacuum. The crude products were purified by flash chromatography to afford pure $16 \mathbf{i}$.

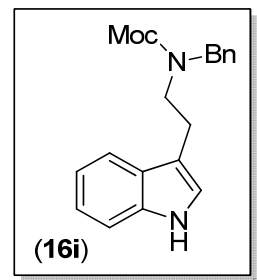

Methyl (2-(1H-indol-3-yl)ethyl)(benzyl)carbamate (16i): 1.57 gm (overall yield 85\% in 2 steps) as white solid. $\mathrm{R}_{f}=0.45\left(30 \%\right.$ EtOAc in hexane). ${ }^{1} \mathbf{H}$ NMR $\left(400 \mathrm{MHz}, \mathrm{CDCl}_{3}\right)$ (rotameric mixture) $\delta: 8.04$ (brs, $1 \mathrm{H}), 7.50-7.55(\mathrm{~m}, 1 \mathrm{H}), 7.29-7.34(\mathrm{~m}, 3 \mathrm{H}), 7.23-7.27$ (m, 2H), $7.18(\mathrm{t}, J=6.9 \mathrm{~Hz}, 2 \mathrm{H}), 7.10(\mathrm{t}, J=7.4 \mathrm{~Hz}, 1 \mathrm{H}), 6.92-6.96(\mathrm{~m}, 1 \mathrm{H}), 4.46(\mathrm{~s}$, 
$1.09 \mathrm{H}), 4.42(\mathrm{~s}, 0.91 \mathrm{H}), 3.76(\mathrm{~s}, 1.41 \mathrm{H}), 3.74(\mathrm{~s}, 1.59 \mathrm{H}), 3.47-3.55(\mathrm{~m}, 2 \mathrm{H}), 2.93-2.99$ $(\mathrm{m}, 2 \mathrm{H}) ;{ }^{13} \mathbf{C}$ NMR $\left(100 \mathrm{MHz}, \mathrm{CDCl}_{3}\right)$ (rotameric mixture) $\delta: 157.0,138.0,136.3,128.6$, 128.0, 127.3, 122.0, 121.9, 119.4, 118.8, 118.7, 113.1, 111.2, 52.7, 50.9, 50.8, 48.0, 47.0, 24.4, 23.8; IR (film) $v_{\max } 3330,3029,2954,1685,1435,1248,1116,742 \mathrm{~cm}^{-1}$; MP $65-67{ }^{\circ} \mathrm{C}$

\section{Procedure for synthesis of (16j):}

An oven-dried round-bottom flask was charged with $N$-benzyl tryptamine $\mathbf{1 6 h}(6.0 \mathrm{mmol}$; 1.0 equiv.) in $\mathrm{CH}_{2} \mathrm{Cl}_{2}(20 \mathrm{~mL})$ under argon atmosphere and the reaction vessel was cooled to $0{ }^{\circ} \mathrm{C}$. To this reaction mixture $\mathrm{Et}_{3} \mathrm{~N}(1.25 \mathrm{~mL}, 9.0 \mathrm{mmol} ; 1.5$ equiv), allyl chloroformate (637 $\mu \mathrm{L}, 6.0 \mathrm{mmol} ; 1.0$ equiv) and DMAP (73 mg, $0.6 \mathrm{mmol} ; 0.1$ equiv) was added and it was stirred for overnight. Upon completion of the reactions, (TLC showed complete consumption of starting material) the reaction mixture was quenched with water $(30 \mathrm{~mL})$ and diluted with another $30 \mathrm{~mL}$ of $\mathrm{CH}_{2} \mathrm{Cl}_{2}$. The whole reaction mixture was taken in a separatory funnel and extracted the organic layer. The organic filtrate was dried over anhydrous $\mathrm{Na}_{2} \mathrm{SO}_{4}$ and concentrated in a rotary evaporator under vacuum. The crude products were purified by flash chromatography to afford pure $\mathbf{1 6} \mathbf{j}$.

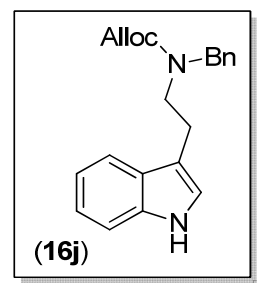

Allyl (2-(1H-indol-3-yl)ethyl)(benzyl)carbamate (16j): 1.75 gm (overall yield 85\% in 2 steps) as colorless gel. $\mathrm{R}_{f}=0.60\left(30 \%\right.$ EtOAc in hexane). ${ }^{1} \mathbf{H} \mathbf{~ N M R}\left(400 \mathrm{MHz}, \mathrm{CDCl}_{3}\right)$ (rotameric mixture) $\delta: 8.18(\mathrm{~s}, 1 \mathrm{H}), 7.54-7.59(\mathrm{~m}, 1 \mathrm{H}), 7.18-7.35(\mathrm{~m}, 7 \mathrm{H}), 7.12(\mathrm{t}, J=7.4$ Hz, 1H), $6.92(\mathrm{~m}, 1 \mathrm{H}), 5.93-5.99(\mathrm{~m}, 1 \mathrm{H}), 5.21-5.37(\mathrm{~m}, 2 \mathrm{H}), 4.68-4.70(\mathrm{~m}, 2 \mathrm{H}), 4.49(\mathrm{~s}$, 2H), 3.51-3.59 (m, 2H), 2.98-3.03 (m, 2H); ${ }^{13} \mathbf{C}$ NMR (100 MHz, $\left.\mathrm{CDCl}_{3}\right)($ rotameric mixture) $\delta: 156.7,156.2,138.0,136.3,133.1,128.6,128.0,127.6,127.4,127.0,122.0$, 119.3, 118.7, 117.5, 117.4, 113.0, 112.9, 111.3, 66.3, 66.1, 51.0, 50.8, 48.1, 47.1, 24.4, 23.8; IR (film) $v_{\max } 3521,2949,1648,1419,1246,1106,982,740 \mathrm{~cm}^{-1}$. 


\section{Procedure for synthesis of (16k):}

An oven-dried round-bottom flask was charged with $N$-benzyl tryptamine $\mathbf{1 6 h}(6.0 \mathrm{mmol}$; 1.0 equiv.) in $\mathrm{CH}_{2} \mathrm{Cl}_{2}(20 \mathrm{~mL})$ under argon atmosphere and the reaction vessel was cooled to $0{ }^{\circ} \mathrm{C}$. To this reaction mixture $\mathrm{Et}_{3} \mathrm{~N}$ (1.25 mL, $9.0 \mathrm{mmol} ; 1.5$ equiv), $p$-TsCl (1.14 gm, $6.0 \mathrm{mmol} ; 1.0$ equiv) and DMAP (73 mg, $0.6 \mathrm{mmol} ; 0.1$ equiv) was added and it was stirred for overnight. Upon completion of the reactions, (TLC showed complete consumption of starting material) the reaction mixture was quenched with water $(30 \mathrm{~mL})$ and diluted with another $30 \mathrm{~mL}$ of $\mathrm{CH}_{2} \mathrm{Cl}_{2}$. The whole reaction mixture was taken in a separatory funnel and extracted the organic layer. The organic filtrate was dried over anhydrous $\mathrm{Na}_{2} \mathrm{SO}_{4}$ and concentrated in a rotary evaporator under vacuum. The crude products were purified by flash chromatography to afford pure 16k.

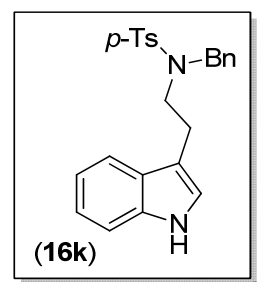

$N$-(2-(1H-indol-3-yl)ethyl)- $N$-benzyl-4-methylbenzenesulfonamide $\quad$ (16k): $2.01 \quad \mathrm{~g}$ (overall yield $83 \%$ in 2 steps) as white crystalline solid. $\mathrm{R}_{f}=0.25(30 \%$ EtOAc in hexane). ${ }^{1} \mathbf{H}$ NMR (400 MHz, $\mathrm{CDCl}_{3}$ ) $\delta: 7.96$ (brs, $\left.1 \mathrm{H}\right), 7.74(\mathrm{~d}, J=8.2 \mathrm{~Hz}, 2 \mathrm{H}), 7.23$ $7.32(\mathrm{~m}, 9 \mathrm{H}), 7.14(\mathrm{t}, J=7.3 \mathrm{~Hz}, 1 \mathrm{H}), 7.02(\mathrm{t}, J=7.3 \mathrm{~Hz}, 1 \mathrm{H}), 6.80(\mathrm{~d}, J=1.8 \mathrm{~Hz}, 1 \mathrm{H})$, $4.37(\mathrm{~s}, 2 \mathrm{H}), 3.35(\mathrm{t}, J=8.0 \mathrm{~Hz}, 2 \mathrm{H}), 2.81(\mathrm{t}, J=8.4 \mathrm{~Hz}, 2 \mathrm{H}), 2.42(\mathrm{~s}, 3 \mathrm{H}) ;{ }^{13} \mathrm{C}$ NMR $\left(100 \mathrm{MHz}, \mathrm{CDCl}_{3}\right) \delta 143.3,137.0,136.5,136.2,129.8,128.7,128.5,127.9,127.2,127.1$, 122.0, 121.97, 119.3, 118.63, 112.6, 111.2, 52.5, 48.7, 25.1, 21.5; IR (film) $v_{\max } 3410$, $3060,2926,2866,1338,1156,1096,935,815,744 \mathrm{~cm}^{-1}$; MP $85-87^{\circ} \mathrm{C}$.

\section{General Procedure for Synthesis of (8f-h):}




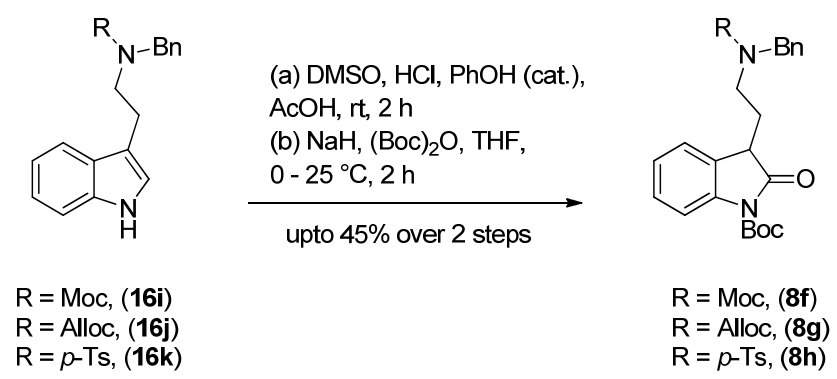

\section{Step 1:}

In an oven-dried round-bottom flask, the protected $N$-benzyl tryptamines $\mathbf{1 6} \mathbf{i}-\mathbf{k}$ ( $4.5 \mathrm{mmol} ; 1.0$ equiv) was taken in acetic acid $(12 \mathrm{~mL})$. To this reaction mixture dimethyl sulphoxide (799 $\mu \mathrm{L}, 11.25 \mathrm{mmol} ; 2.5$ equiv), $12(N) \mathrm{HCl}(1.87 \mathrm{~mL} ; 22.5 \mathrm{mmol}, 5.0$ equiv) and phenol (21 mg, $0.225 \mathrm{mmol} ; 0.05$ equiv) was added respectively and it was stirred for $2 \mathrm{~h}$ at $\mathrm{rt}$. Upon completion of the reactions, (TLC showed complete consumption of starting material) the reaction mixture was quenched with saturated $2(\mathrm{M})$ $\mathrm{NaOH}$ soln. and then diluted with $50 \mathrm{~mL}$ of EtOAc. The whole reaction mixture was taken in a separatory funnel and extracted with $20 \mathrm{~mL}$ of water. The organic filtrate was dried over $\mathrm{Na}_{2} \mathrm{SO}_{4}$ and concentrated in a rotary evaporator under vacuum. The crude products were purified by flash chromatography to afford 18d-f.

\section{Step 2:}

In an oven-dried round-bottom flask, the compound 18d-f (1.0 mmol; 1.0 equiv) was taken in dry THF $(10 \mathrm{~mL})$ under argon atmosphere at $0{ }^{\circ} \mathrm{C}$. To this reaction mixture $\mathrm{NaH}$ (49 mg, $1.1 \mathrm{mmol}$; 1.1 equiv) was added portionwise and it was stirred for $5 \mathrm{~min}$. Boc-anhydride (241 $\mu \mathrm{L}, 1.05 \mathrm{mmol}, 1.05$ equiv) was added to the reaction mixture dropwise at $0{ }^{\circ} \mathrm{C}$ and it was stirred for $2 \mathrm{~h}$ at RT. Upon completion of the reactions, (TLC showed complete consumption of starting material) the reaction mixture was quenched with ice-water and then diluted with $20 \mathrm{~mL}$ of EtOAc. The whole reaction mixture was taken in a separatory funnel and extracted with $15 \mathrm{~mL}$ of water. The organic filtrate was dried over $\mathrm{Na}_{2} \mathrm{SO}_{4}$ and concentrated in a rotary evaporator under vacuum. The crude products were purified by flash chromatography to afford $\mathbf{8 f - h}$. 


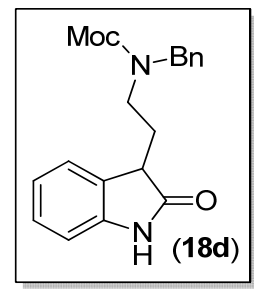

Methyl benzyl(2-(2-oxoindolin-3-yl)ethyl)carbamate (18d): Yellow gel. $\mathrm{R}_{f}=0.25$ (50\% EtOAc in hexane). ${ }^{1} \mathbf{H}$ NMR $\left(400 \mathrm{MHz}, \mathrm{CDCl}_{3}\right.$ ) (rotameric mixture) $\delta: 8.99$ (brs, 0.5H), 8.92 (brs, 0.5H), 7.19-7.29 (m, 7H), 7.01 (t, $J=7.5 \mathrm{~Hz}, 1 \mathrm{H}), 6.87$ (d, $J=7.5 \mathrm{~Hz}$, 1H), 4.54 (d, $J=15.5 \mathrm{~Hz}, 1 \mathrm{H}), 4.35(\mathrm{~d}, J=15.2 \mathrm{~Hz}, 1 \mathrm{H}), 3.69$ (s, 3H), 3.57 (brs, $0.51 \mathrm{H})$, 3.43 (brs, 1H), 3.28 (m, 1.49H), 2.17 (m, 2H); ${ }^{13}$ C NMR (100 MHz, $\left.\mathrm{CDCl}_{3}\right) \delta 180.2$, 180.0, 175.0, 157.2, 157.0, 141.7, 137.7, 129.1, 128.9, 128.6, 128.0, 127.4, 124.3, 123.9, $122.5,122.4,109.9,109.8,52.8,50.4,50.2,43.8,28.4,28.3,21.1,20.9$; IR (film) $v_{\max }$ $3497,2958,1655,1450,1237,1121,754 \mathrm{~cm}^{-1}$.

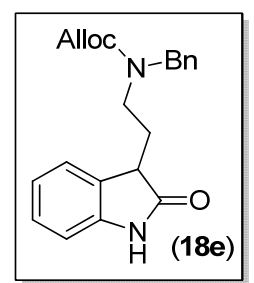

Allyl benzyl(2-(2-oxoindolin-3-yl)ethyl)carbamate (18e): Yellow gel. $\mathrm{R}_{f}=0.28(40 \%$ EtOAc in hexane). ${ }^{1} \mathbf{H}$ NMR $\left(400 \mathrm{MHz}, \mathrm{CDCl}_{3}\right.$ ) (rotameric mixture) $\delta: 8.96$ (brs, $0.5 \mathrm{H}$ ), 8.91 (brs, 0.5H), 7.13-7.28 (m, 7H), 7.0 (t, $J=7.5 \mathrm{~Hz}, 1 \mathrm{H}), 6.86(\mathrm{~d}, J=7.7 \mathrm{~Hz}, 1 \mathrm{H}), 5.90$ (brs, $1 \mathrm{H}), 5.17-5.30(\mathrm{~m}, 2 \mathrm{H}), 4.55-4.64(\mathrm{~m}, 3 \mathrm{H}), 4.34-4.41(\mathrm{~m}, 1 \mathrm{H}), 3.30-3.57(\mathrm{~m}, 3 \mathrm{H})$, 2.15-2.19 (m, 2H); ${ }^{13} \mathrm{C}$ NMR $\left(100 \mathrm{MHz}, \mathrm{CDCl}_{3}\right.$ ) (rotameric mixture) $\delta: 180.3,180.0$, $175.2,156.4,156.1,141.5,141.4,137.7,132.9,128.6,128.0,127.5,127.4,124.3$, 124.0, $122.48,122.45,117.5,109.9,109.8,66.23,66.19,60.4,60.0,50.5,50.2$, 43.8, 43.1, 21.1, 20.9; IR (film) $v_{\max } 3452,2941,1670,1420,1234,1124,983,751 \mathrm{~cm}^{-1}$. 


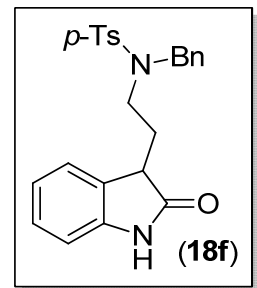

$N$-Benzyl-4-methyl- $N$-(2-(2-oxoindolin-3-yl)ethyl)benzenesulfonamide (18f): Yellow solid. $\mathrm{R}_{f}=0.30$ (40\% EtOAc in hexane). ${ }^{1} \mathbf{H}$ NMR (400 MHz, $\left.\mathrm{CDCl}_{3}\right) \delta: 8.62(\mathrm{~s}, 1 \mathrm{H})$, $7.68(\mathrm{~d}, J=8.2 \mathrm{~Hz}, 2 \mathrm{H}), 7.14-7.26(\mathrm{~m}, 7 \mathrm{H}), 7.14-7.18(\mathrm{~m}, 1 \mathrm{H}), 6.95-6.96(\mathrm{~m}, 2 \mathrm{H}), 6.81$ $(\mathrm{d}, J=7.7 \mathrm{~Hz}, 1 \mathrm{H}), 4.31(\mathrm{ABq}, J=30.8,14.7 \mathrm{~Hz}, 2 \mathrm{H}), 3.29$ (t, $J=6.3 \mathrm{~Hz}, 1 \mathrm{H}), 3.23$ (t, $J$ $=7.9 \mathrm{~Hz}, 2 \mathrm{H}), 2.40(\mathrm{~s}, 3 \mathrm{H}), 1.89-2.0(\mathrm{~m}, 2 \mathrm{H}) ;{ }^{13} \mathbf{C ~ N M R}\left(100 \mathrm{MHz}, \mathrm{CDCl}_{3}\right) \delta 179.6$, $143.4,141.3,136.6,136.2,129.8,128.63,128.61,128.58,128.1,127.9,127.3,124.0$, 122.5, 109.7, 52.4, 45.0, 43.4, 29.3, 21.5; IR (film) $v_{\max }$ 3526, 2953, 1655, 1334, 1158, $1106,937,808,750 \mathrm{~cm}^{-1} ; \mathbf{M P} 80-82{ }^{\circ} \mathrm{C}$.

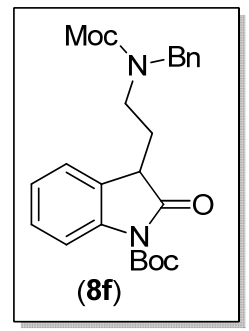

tert-Butyl 3-(2-(benzyl(methoxycarbonyl)amino)ethyl)-2-oxoindoline-1-carboxylate (8f): $187 \mathrm{mg}$ (overall yield $44 \%$ in 2 steps) as colorless gel. $\mathrm{R}_{f}=0.55$ (20\% EtOAc in hexane). ${ }^{1} \mathbf{H}$ NMR (400 MHz, $\mathrm{CDCl}_{3}$ ) (rotameric mixture) $\delta: 7.78(\mathrm{~s}, 0.5 \mathrm{H}), 7.76(\mathrm{~s}$, $0.5 \mathrm{H}), 7.14-7.31(\mathrm{~m}, 8 \mathrm{H}), 4.52(\mathrm{~d}, J=15.6 \mathrm{~Hz}, 1 \mathrm{H}), 4.34(\mathrm{~d}, J=15.5 \mathrm{~Hz}, 1 \mathrm{H}), 3.68$ (s, $3 \mathrm{H}), 3.27-3.51(\mathrm{~m}, 3 \mathrm{H}), 2.12-2.25(\mathrm{~m}, 2 \mathrm{H}), 1.62(\mathrm{~s}, 9 \mathrm{H}) ;{ }^{13} \mathrm{C}$ NMR $\left(100 \mathrm{MHz}, \mathrm{CDCl}_{3}\right)$ (rotameric mixture) $\delta: 175.8,175.5,157.0,156.9,149.1,140.0,139.9,137.6,128.6$, $128.2,128.0,127.4,124.4,123.84,123.80,123.44,123.41,115.0,84.4,52.8,50.5,50.3$, 44.1, 43.7, 43.0, 42.0, 29.1, 28.1, 27.7; IR (film) $v_{\max }$ 2983, 1735, 1703, 1465, 1251, $1151,842,754 \mathrm{~cm}^{-1}$. 


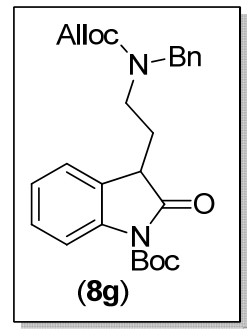

tert-Butyl

carboxylate (8g): $180 \mathrm{mg}$ (overall yield $40 \%$ in 2 steps) as colorless gel. $\mathrm{R}_{f}=0.6(20 \%$ EtOAc in hexane). ${ }^{1} \mathbf{H}$ NMR (400 MHz, $\left.\mathrm{CDCl}_{3}\right) \delta$ : (rotameric mixture) 7.78 (brs, $0.5 \mathrm{H}$ ), 7.76 (brs, 0.5H), 7.13-7.31 (m, 8H), 5.90 (brs, 1H), 5.18-5.29 (m, 2H), 4.54-4.59 (m, 3H), 4.34-4.38 (m, 1H), 3.29-3.52 (m, 3H), 2.13-2.26 (m, 2H), $1.62(\mathrm{~s}, 9 \mathrm{H}) ;{ }^{13}$ C NMR (100 $\mathrm{MHz}, \mathrm{CDCl}_{3}$ ) (rotameric mixture) $\delta: 175.9,175.5,156.3,156.2,149.1,140.02,139.97$, $137.6,132.9,128.6,128.3,128.25,128.23,128.16,128.11,128.09,127.43,127.36$, $124.45,124.41,124.38,123.9,123.5,117.5,115.98,114.96,84.4,66.2,50.61,50.57$, 50.32, 50.28, 43.7, 28.1, 20.80, 20.77; IR (film) $v_{\max } 2983,2936,1734,1417,1292$, $1250,1151,755 \mathrm{~cm}^{-1}$.

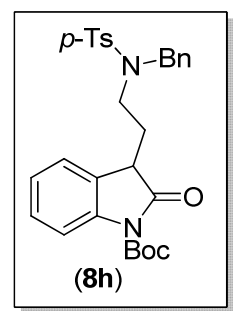

tert-Butyl 3-(2-(( $N$-benzyl-4-methylphenyl)sulfonamido)ethyl)-2-oxoindoline-1carboxylate (8h): $234 \mathrm{mg}$ (overall yield $45 \%$ in 2 steps) as colorless gel. $\mathrm{R}_{f}=0.35(20 \%$ EtOAc in hexane). ${ }^{1} \mathbf{H}$ NMR (400 MHz, $\left.\mathrm{CDCl}_{3}\right) \delta:$ 7.67-7.72 (m, 3H), 7.22-7.29 (m, 8H), $7.07(\mathrm{t}, J=7.5 \mathrm{~Hz}, 1 \mathrm{H}), 6.95(\mathrm{~d}, J=7.4 \mathrm{~Hz}, 1 \mathrm{H}), 4.31(\mathrm{ABq}, J=43.4,14.7 \mathrm{~Hz}, 2 \mathrm{H})$, $3.89(\mathrm{t}, J=6.3 \mathrm{~Hz}, 1 \mathrm{H}), 3.23-3.27(\mathrm{~m}, 2 \mathrm{H}), 2.41(\mathrm{~s}, 3 \mathrm{H}), 1.89-2.0(\mathrm{~m}, 2 \mathrm{H}), 1.61(\mathrm{~s}, 9 \mathrm{H})$; ${ }^{13} \mathrm{C}$ NMR $\left(100 \mathrm{MHz}, \mathrm{CDCl}_{3}\right) \delta 175.5,149.1,143.4,139.8,136.5,136.3,129.8,128.7$, $128.6,128.2$, 127.9, 127.3, 126.9, 124.4, 123.6, 114.8, 84.3, 52.7, 45.3, 43.4, 30.1, 28.1, 21.5; IR (film) $v_{\max } 2982,2933,1655,1293,1253,1156,939,755 \mathrm{~cm}^{-1}$.

\section{Synthesis of 8i and 81:}




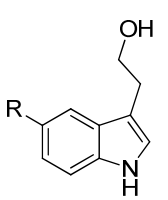

$\mathrm{R}=\mathrm{H}, \mathbf{1 6}$ $\mathrm{R}=\mathrm{OMe}, 16 \mathrm{~m}$
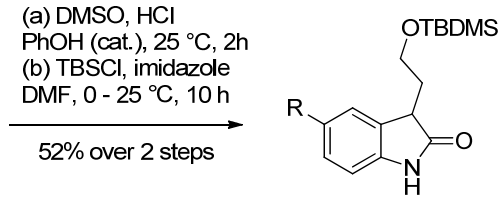

$\underset{\left.\mathrm{Na}, 65^{\circ} \mathrm{CO}_{3}, 8 \mathrm{Boc}\right)_{2} \mathrm{O}}{\stackrel{\mathrm{TH}^{2}}{\longrightarrow}}$
$72 \%$ yield

$\mathrm{R}=\mathrm{H}, \mathbf{1 8 g}$

$\mathrm{R}=\mathrm{OMe}, 18 \mathrm{~h}$

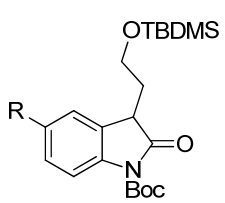

$\mathrm{R}=\mathrm{H}, \mathbf{8} \mathbf{i}$

$\mathrm{R}=\mathrm{OMe}, \mathbf{8 I}$

\section{Procedure for synthesis of (18g-h):}

Step 1: In an oven-dried round-bottom flask, the tryptophol (1.0 gm scale; 1.0 equiv) was taken in acetic acid $(10 \mathrm{~mL})$. To this reaction mixture dimethyl sulphoxide ( 2.5 equiv), 12 $(N) \mathrm{HCl}$ (5.0 equiv) and phenol (0.05 equiv) was added respectively and it was stirred for $2 \mathrm{~h}$ at room temperature. Upon completion of the reactions, (TLC showed complete consumption of starting material) the reaction mixture was quenched with saturated $2(\mathrm{M})$ $\mathrm{NaOH}$ soln. and then diluted with $50 \mathrm{~mL}$ of EtOAc. The whole reaction mixture was taken in a separatory funnel and extracted with $20 \mathrm{~mL}$ of water. The organic filtrate was dried over $\mathrm{Na}_{2} \mathrm{SO}_{4}$ and concentrated in a rotary evaporator under vacuum. The crude products were purified by flash chromatography and without isolation it was directly treated for next step.

Step 2: In an oven-dried round-bottom flask, the DMSO treatment product (1.0 equiv) was taken in $N, N$-dimethylformamide $(7 \mathrm{~mL})$. To this reaction mixture imidazole $(2.0$ equiv) and TBSCl (1.1 equiv) was added respectively and it was stirred for $2 \mathrm{~h}$ at $\mathrm{rt}$. Upon completion of the reactions, (TLC showed complete consumption of starting material) the reaction mixture was diluted with $20 \mathrm{~mL}$ of EtOAc. The whole reaction mixture was taken in a separatory funnel and extracted with $40 \mathrm{~mL}$ of water. The organic filtrate was dried over $\mathrm{Na}_{2} \mathrm{SO}_{4}$ and concentrated in a rotary evaporator under vacuum. The crude products were purified by flash chromatography to afford 18g-h.

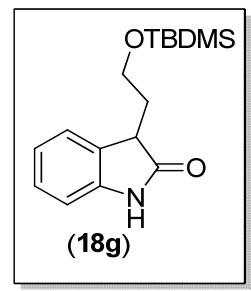


3-(2-((tert-Butyldimethylsilyl)oxy)ethyl)indolin-2-one (18g): $936 \mathrm{mg}$ (overall yield $52 \%$ in 2 steps) as colorless liquid. $\mathrm{R}_{f}=0.30$ (20\% EtOAc in hexane). ${ }^{\mathbf{1}} \mathbf{H}$ NMR (400 $\left.\mathrm{MHz} \mathrm{CDCl}_{3}\right) \delta: 8.63(\mathrm{brs}, 1 \mathrm{H}), 7.17-7.24(\mathrm{~m}, 2 \mathrm{H}), 7.0(\mathrm{t}, J=7.4 \mathrm{~Hz}, 1 \mathrm{H}), 6.87(\mathrm{~d}, J=$ $7.7 \mathrm{~Hz}, 1 \mathrm{H}), 3.77-3.83(\mathrm{~m}, 2 \mathrm{H}), 3.60(\mathrm{t}, J=6.4 \mathrm{~Hz}, 1 \mathrm{H}), 2.18-2.23(\mathrm{~m}, 1 \mathrm{H}), 2.05-2.10$ $(\mathrm{m}, 1 \mathrm{H}), 1.71(\mathrm{brs}, 1 \mathrm{H}), 0.85(\mathrm{~s}, 9 \mathrm{H}), 0.003(\mathrm{~s}, 3 \mathrm{H}),-0.01(\mathrm{~s}, 3 \mathrm{H})$; IR (film) $v_{\max } 3325$, $2955,2932,2862,1716,1255,1191,1109,837,753 \mathrm{~cm}^{-1}$.

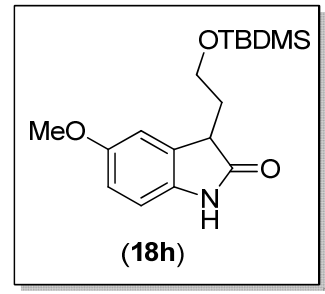

3-(2-((tert-butyldimethylsilyl)oxy)ethyl)-5-methoxyindolin-2-one (18h): $926 \mathrm{mg}$ (overall yield 55\% in 2 steps) as white solid. $\mathrm{R}_{f}=0.30$ (30\% EtOAc in hexane). ${ }^{1} \mathbf{H}$ NMR $\left(400 \mathrm{MHz}, \mathrm{CDCl}_{3}\right) \delta: 8.95$ (brs, $\left.1 \mathrm{H}\right), 6.85(\mathrm{~s}, 1 \mathrm{H}), 6.79$ (d, $\left.J=8.4 \mathrm{~Hz}, 1 \mathrm{H}\right), 6.72(\mathrm{dd}, J=$ 2.3, 8.4 Hz, 1H), 3.78-3.83 (m, 2H), $3.76(\mathrm{~s}, 3 \mathrm{H}), 3.58(\mathrm{t}, J=6.4 \mathrm{~Hz}, 1 \mathrm{H}), 2.24-2.16(\mathrm{~m}$, 1H), 2.01-2.10 (m, 1H), $0.85(\mathrm{~s}, 9 \mathrm{H}), 0.01(\mathrm{~s}, 3 \mathrm{H}),-0.001(\mathrm{~s}, 3 \mathrm{H}) ;{ }^{13} \mathrm{C}$ NMR $(100 \mathrm{MHz}$, $\left.\mathrm{CDCl}_{3}\right) \delta 180.9,155.6,135.2,131.0,112.3,111.7,109.9,59.7,55.8,43.4,33.3,25.9$, 18.3, -5.4, -5.5; IR (film) $v_{\max } 3270,2939,2855,1710,1605,1490,1256,1208,1102$, $1072,1033,836 \mathrm{~cm}^{-1}$; MP $79-80{ }^{\circ} \mathrm{C}$.

\section{Procedure for synthesis of $8 \mathrm{i}$ and 81 :}

In an oven-dried round-bottom flask, the compound $\mathbf{1 8 g}-\mathbf{h}$ (250 $\mathrm{mg}$ scale, 1.0 equiv) was taken in dry THF $(10 \mathrm{~mL})$ under argon atmosphere at room temperature. To this reaction mixture $\mathrm{Na}_{2} \mathrm{CO}_{3}$ (8.0 equiv) and Boc-anhydride (1.2 equiv) was added respectively and it was stirred for $14 \mathrm{~h}$ at $65^{\circ} \mathrm{C}$. Upon completion of the reactions, (TLC showed complete consumption of starting material) the reaction mixture was quenched with ice-water and then diluted with $20 \mathrm{~mL}$ of EtOAc. The whole reaction mixture was taken in a separatory funnel and extracted with $15 \mathrm{~mL}$ of water. The organic filtrate was dried over $\mathrm{Na}_{2} \mathrm{SO}_{4}$ and concentrated in a rotary evaporator under vacuum. The crude products were purified by flash chromatography to afford $\mathbf{8 i}-\mathbf{l}$. 


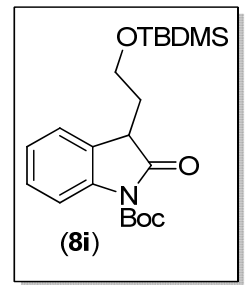

tert-Butyl 3-(2-((tert-butyldimethylsilyl)oxy)ethyl)-2-oxoindoline-1-carboxylate (8i): $242 \mathrm{mg}$ (72\% yields) as colorless gel. $\mathrm{R}_{f}=0.60$ (10\% EtOAc in hexane). ${ }^{\mathbf{1}} \mathbf{H}$ NMR (400 $\left.\mathrm{MHz}, \mathrm{CDCl}_{3}\right) \delta: 7.80(\mathrm{~d}, J=8.2 \mathrm{~Hz}, 1 \mathrm{H}), 7.21-7.28(\mathrm{~m}, 2 \mathrm{H}), 7.12(\mathrm{t}, J=7.4 \mathrm{~Hz}, 1 \mathrm{H})$, 3.71-3.75 (m, 2H), $3.67(\mathrm{t}, J=5.8 \mathrm{~Hz}, 1 \mathrm{H}), 2.16-2.22(\mathrm{~m}, 2 \mathrm{H}), 1.62(\mathrm{~s}, 9 \mathrm{H}), 0.80(\mathrm{~s}, 9 \mathrm{H})$, -0.06 (s, 3H), - 0.07 (s, 3H); ${ }^{13}$ C NMR (100 MHz, $\left.\mathrm{CDCl}_{3}\right) \delta$ 176.3, 149.4, 140.3, 127.9, 127.6, 124.0, 123.8, 115.0, 84.0, 59.3, 42.8, 33.4, 28.1, 25.9, 18.2, -5.59, -5.6; IR (film) $v_{\max } 2957,1730,1649,1468,1349,1293,1253,1151,1112,837,775 \mathrm{~cm}^{-1}$.

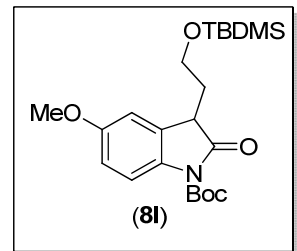

tert-Butyl 3-(2-((tert-butyldimethylsilyl)oxy)ethyl)-5-methoxy-2-oxoindoline-1carboxylate (81): $229 \mathrm{mg}$ (70\% yields) as colorless gel. $\mathrm{R}_{f}=0.50(10 \%$ EtOAc in hexane). ${ }^{1} \mathbf{H}$ NMR $\left(400 \mathrm{MHz}, \mathrm{CDCl}_{3}\right) \delta: 7.71(\mathrm{~d}, J=8.6 \mathrm{~Hz}, 1 \mathrm{H}), 6.77-6.79(\mathrm{~m}, 2 \mathrm{H})$, $3.77(\mathrm{~s}, 3 \mathrm{H}), 3.73(\mathrm{t}, J=5.8 \mathrm{~Hz}, 2 \mathrm{H}), 3.64(\mathrm{t}, J=6.0 \mathrm{~Hz}, 1 \mathrm{H}), 2.14-2.19$ (m, 2H), 1.60 (s, 9H), $0.81(\mathrm{~s}, 9 \mathrm{H}),-0.05$ (s, 3H), $-0.06(\mathrm{~s}, 3 \mathrm{H}) ;{ }^{13} \mathrm{C}$ NMR $\left(100 \mathrm{MHz}, \mathrm{CDCl}_{3}\right) \delta 176.3$, 156.6, 149.4, 133.7, 129.0, 115.8, 112.5, 110.4, 83.8, 59.3, 55.6, 43.1, 33.5, 28.1, 27.4, 25.9, 18.2, -5.5, -5.6; IR (film) $v_{\max }$ 2938, 2859, 1794, 1772, 1727, 1494, 1476, 1370, $1341,1304,1288,1251,1167,1156,1110,1075,1007,836 \mathrm{~cm}^{-1}$.

\section{Synthesis of 8j-k:}




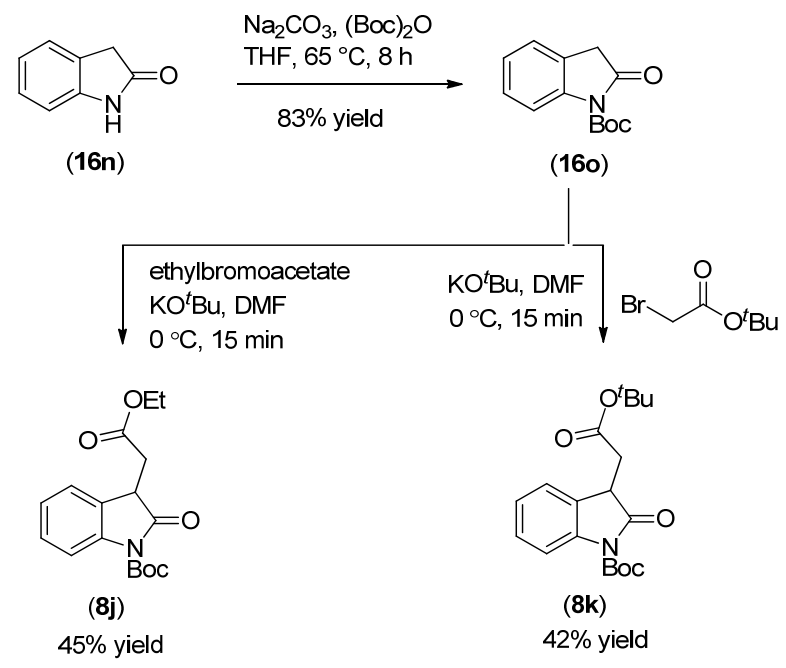

\section{Synthesis and characterization of (16o):}

In an oven-dried round-bottom flask, the 2-oxindole 16n $(5.045 \mathrm{gm} ; 37.89 \mathrm{mmol} ; 1.0$ equiv) was taken in dry THF $(150 \mathrm{~mL})$ under argon atmosphere at $0{ }^{\circ} \mathrm{C}$. To this reaction mixture $\mathrm{Na}_{2} \mathrm{CO}_{3}$ (32.4 gm, 303.12 mmol; 8.0 equiv) and Boc-anhydride (13.05 mL, 56.83 mmol; 1.5 equiv) was added respectively and it was stirred for $8 \mathrm{~h}$ at $65^{\circ} \mathrm{C}$. Upon completion of the reactions, (TLC showed complete consumption of starting material) the reaction mixture was quenched with ice-water and then diluted with $100 \mathrm{~mL}$ of EtOAc. The reaction mixture was filtered and the filtration was concentrated in a rotary evaporator under vacuum. The crude products were purified by flash chromatography to afford 160.

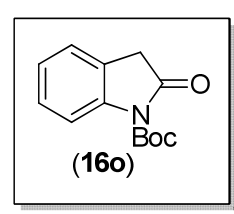

tert-Butyl 2-oxoindoline-1-carboxylate (16o): $7.4 \mathrm{~g}$ (83\% yields) as yellow solid. $\mathrm{R}_{f}=$ $0.50\left(10 \%\right.$ EtOAc in hexane). ${ }^{1} \mathbf{H}$ NMR $\left(400 \mathrm{MHz} \mathrm{CDCl}_{3}\right) \delta: 7.76(\mathrm{~d}, J=8.2 \mathrm{~Hz}, 1 \mathrm{H})$, $7.27(\mathrm{t}, J=7.8 \mathrm{~Hz}, 1 \mathrm{H}), 7.21(\mathrm{~d}, J=7.2 \mathrm{~Hz}, 1 \mathrm{H}), 7.1(\mathrm{t}, J=7.4 \mathrm{~Hz}, 1 \mathrm{H}), 3.62(\mathrm{~s}, 2 \mathrm{H})$, $1.62(\mathrm{~s}, 9 \mathrm{H}) ;{ }^{13} \mathrm{C}$ NMR $\left(100 \mathrm{MHz}, \mathrm{CDCl}_{3}\right) \delta 173.1,149.2,141.0,128.1,124.2,123.2$, 115.0, 84.3, 36.5, 28.1; IR (film) $v_{\max }$ 2982, 2935, 1769, 1730, 1480, 1467, 1394, 1341, $1294,1255,1168,1090,826,774,754 \mathrm{~cm}^{-1}$; MP 59-61 ${ }^{\circ} \mathrm{C}$. 


\section{General Procedure for Synthesis of $(\mathbf{8 j}-\mathbf{k})$ :}

In an oven-dried round-bottom flask, the Boc-protected 2-oxindole $160(1.028 \mathrm{mmol} ; 1.0$ equiv) was taken in $\mathrm{N}, \mathrm{N}$-dimethylformamide $(5 \mathrm{~mL})$ under argon atmosphere and the reaction vessel was cooled to $-5{ }^{\circ} \mathrm{C}$. To this reaction mixture potassium-tert-butoxide (127 mg, $1.131 \mathrm{mmol} ; 1.1$ equiv) was added and it was stirred for $5 \mathrm{~min}$. To the reaction mixture, ethyl 2-bromoacetate or tert-butyl bromoacetate $(1.079 \mathrm{mmol} ; 1.05$ equiv) in $N$, $N$-dimethylformamide $(2 \mathrm{~mL})$ was added dropwise to the reaction mixture at $0{ }^{\circ} \mathrm{C}$ for 15 min. Then the reaction mixture was quenched with saturated ice-water $(3 \mathrm{~mL})$ and then diluted with $10 \mathrm{~mL}$ of EtOAc. The whole reaction mixture was taken in a separatory funnel and extracted with $15 \mathrm{~mL}$ of water. The organic filtrate was dried over anhydrous $\mathrm{Na}_{2} \mathrm{SO}_{4}$ and concentrated in a rotary evaporator under vacuum. The crude products were purified by flash chromatography to afford $\mathbf{8 j} \mathbf{j} \mathbf{k}$.

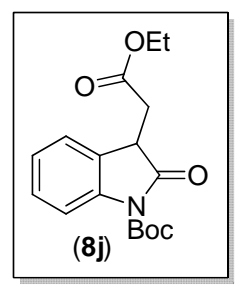

tert-Butyl 3-(2-ethoxy-2-oxoethyl)-2-oxoindoline-1-carboxylate (8j): $147 \mathrm{mg}$ (45\% yields) as yellow oil. $\mathrm{R}_{f}=0.45\left(10 \%\right.$ EtOAc in hexane) ${ }^{1} \mathbf{H} \mathbf{~ N M R}\left(400 \mathrm{MHz}, \mathrm{CDCl}_{3}\right) \delta$ : $7.80(\mathrm{~d}, J=8.2 \mathrm{~Hz}, 1 \mathrm{H}), 7.28$ (t, $J=7.7 \mathrm{~Hz}, 1 \mathrm{H}), 7.20$ (d, $J=7.4 \mathrm{~Hz}, 1 \mathrm{H}), 7.09-7.12$ (m, $1 \mathrm{H}), 4.04-4.10(\mathrm{~m}, 2 \mathrm{H}), 3.84-3.90(\mathrm{~m}, 2 \mathrm{H}), 3.04(\mathrm{dd}, J=17.0,4.6 \mathrm{~Hz}, 1 \mathrm{H}), 2.92-2.95(\mathrm{~m}$, $1 \mathrm{H}), 1.62(\mathrm{~s}, 9 \mathrm{H}), 1.14(\mathrm{t}, J=7.2 \mathrm{~Hz}, 3 \mathrm{H}) ;{ }^{13} \mathbf{C} \mathbf{N M R}\left(100 \mathrm{MHz}, \mathrm{CDCl}_{3}\right) \delta: 175.2,170.5$, $149.2,140.3,128.5,126.9,124.4,123.4,115.0,84.3,61.1,42.5,35.3,28.1,14.0$; IR (film) $v_{\max } 2984,2936,1770,1730,1465,1295,1252,1153,1096,844,756 \mathrm{~cm}^{-1}$.

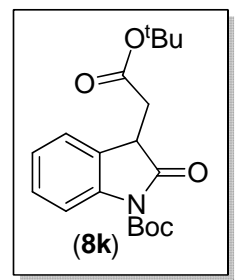


tert-Butyl 3-(2-(tert-butoxy)-2-oxoethyl)-2-oxoindoline-1-carboxylate (8k): $150 \mathrm{mg}$ (42\% yields) as colorless gel. $\mathrm{R}_{f}=0.48\left(30 \%\right.$ EtOAc in hexane). ${ }^{1} \mathbf{H}$ NMR (400 MHz, $\left.\mathrm{CDCl}_{3}\right) \delta: 7.81(\mathrm{~d}, J=8.2 \mathrm{~Hz}, 1 \mathrm{H}), 7.28(\mathrm{t}, J=7.8 \mathrm{~Hz}, 1 \mathrm{H}), 7.21(\mathrm{~d}, J=7.4 \mathrm{~Hz}, 1 \mathrm{H})$, $7.11(\mathrm{t}, J=7.3 \mathrm{~Hz}, 1 \mathrm{H}), 3.76(\mathrm{t}, J=5.3 \mathrm{~Hz}, 1 \mathrm{H}), 2.95(\mathrm{dd}, J=5.6,2.1 \mathrm{~Hz}, 2 \mathrm{H}), 1.62(\mathrm{~s}$, 9H), $1.23(\mathrm{~s}, 9 \mathrm{H}) ;{ }^{13} \mathrm{C}$ NMR $\left(100 \mathrm{MHz}, \mathrm{CDCl}_{3}\right) \delta 175.3,169.2,149.2,140.4,128.4$, 127.0, 124.3, 123.4, 114.9, 84.3, 81.6, 42.7, 36.3, 28.1, 27.6; IR (film) $v_{\max } 2983,1779$, $1734,1351,1295,1252,1152,1095,843,756 \mathrm{~cm}^{-1}$.

\section{General Procedure for organocatalytic Aldol Reaction:}

In a sealed tube equipped with a magnetic stirring bar, to the mixture of $8(0.03$ mmol; 1.0 equiv) and paraformaldehyde solid $(20.0 \mathrm{mg})$ were taken in $0.5 \mathrm{~mL}$ of HPLC grade $\mathrm{CH}_{3} \mathrm{CN}$ and the reaction mixture was kept in $-5{ }^{\circ} \mathrm{C}$. After that $\mathbf{L 7}(0.003 \mathrm{mmol} ; 0.1$ equiv) in was added and reaction mixture was stirred for indicated times. Upon completion of the reactions, (TLC showed complete consumption of starting material) the reaction mixture was directly loaded onto silica gel and purified by flash chromatography to give the desired products 6 .

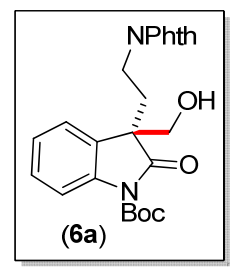

tert-Butyl (S)-3-(2-(1,3-dioxoisoindolin-2-yl)ethyl)-3-(hydroxymethyl)-2-oxoindoline1-carboxylate (6a): $11.5 \mathrm{mg}\left(88 \%\right.$ yields) as white solid. $\mathrm{R}_{f}=0.20(30 \%$ EtOAc in hexane). ${ }^{1} \mathbf{H}$ NMR $\left(400 \mathrm{MHz}, \mathrm{CDCl}_{3}\right) \delta: 7.69(\mathrm{~d}, J=8.1 \mathrm{~Hz}, 1 \mathrm{H}), 7.55-7.62(\mathrm{~m}, 4 \mathrm{H})$, $7.10(\mathrm{~d}, J=7.4 \mathrm{~Hz}, 1 \mathrm{H}), 7.01(\mathrm{td}, J=8.0,1.2 \mathrm{~Hz}, 1 \mathrm{H}), 6.89(\mathrm{t}, J=7.0 \mathrm{~Hz}, 1 \mathrm{H}), 3.71$ (ABq, $J=34.3,10.9 \mathrm{~Hz}, 2 \mathrm{H}), 3.52-3.59(\mathrm{~m}, 1 \mathrm{H}), 3.43-3.5(\mathrm{~m}, 1 \mathrm{H}), 2.30-2.46(\mathrm{~m}, 2 \mathrm{H})$, 2.09 (brs, 1H), 1.56 (s, 9H); ${ }^{13}$ C NMR (100 MHz, $\left.\mathrm{CDCl}_{3}\right) \delta 176.9,167.7,148.9,140.2$, $133.7,131.8,128.5,127.8,124.5,123.0,122.6,115.5,84.4$, 68.3, 53.6, 33.9, 30.6, 28.1; IR (film) $v_{\max } 3476,2982,2936,1715,1647,1513,1260,1171,1147,1025 \mathrm{~cm}^{-1}$; HRMS (ESI) $\mathrm{m} / \mathrm{z} 475.1265[\mathrm{M}+\mathrm{K}]^{+}$; calculated for $\left[\mathrm{C}_{24} \mathrm{H}_{24} \mathrm{~N}_{2} \mathrm{O}_{6}+\mathrm{K}\right]^{+}: 475.1266$; MP 147-149 ${ }^{\circ} \mathrm{C}$; Enantiometric excess was determined to be $91 \%$ ee via HPLC analysis using a 
Chiralpak IB column; solvent: 2-propanol/ hexane $=3 / 17$; flow rate: $1.0 \mathrm{~mL} / \mathrm{min}$; detection: at $254 \mathrm{~nm}): t_{\mathrm{R}}$ major $=14.97 \mathrm{~min}, t_{\mathrm{R}}$ minor $=19.69 \mathrm{~min} .[\alpha]_{589}{ }^{24{ }^{\circ} \mathrm{C}}=+26.09(\mathrm{C}$ $=0.115, \mathrm{MeOH}$, for $91 \%$ ee sample).

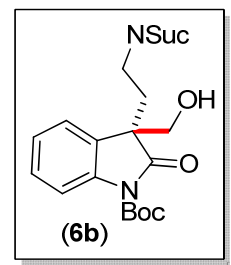

tert-Butyl (S)-3-(2-(2,5-dioxopyrrolidin-1-yl)ethyl)-3-(hydroxymethyl)-2-oxoindoline1-carboxylate (6b): $9.6 \mathrm{mg}\left(83 \%\right.$ yields) as white solid. $\mathrm{R}_{f}=0.10(50 \%$ EtOAc in hexane). ${ }^{1} \mathbf{H}$ NMR $\left(500 \mathrm{MHz}, \mathrm{CDCl}_{3}\right) \delta: 7.89(\mathrm{~d}, J=8.2 \mathrm{~Hz}, 1 \mathrm{H}), 7.34-7.37(\mathrm{~m}, 1 \mathrm{H})$, 7.19-7.24 (m, 2H), 3.71-3.82 (m, 1H), $3.72(\mathrm{~d}, J=10.8 \mathrm{~Hz}, 1 \mathrm{H}), 3.50-3.55(\mathrm{~m}, 1 \mathrm{H})$, 3.33-3.38 (m, 1H), 2.31-2.44 (m, 6H), 2.25 (brs, 1H), $1.68(\mathrm{~s}, 9 \mathrm{H}) ;{ }^{13}$ C NMR (125 MHz, $\left.\mathrm{CDCl}_{3}\right) \delta 177.0,176.9,149.0,140.5,128.9,128.0,124.5,122.5,115.6,84.6,68.4 ;$ IR (film) $v_{\max } 3477,2983,2939,1700,1296,1252,1151,841,756 \mathrm{~cm}^{-1}$; HRMS (ESI) m/z $411.1513[\mathrm{M}+\mathrm{Na}]^{+}$; calculated for $\left[\mathrm{C}_{20} \mathrm{H}_{24} \mathrm{~N}_{2} \mathrm{O}_{6}+\mathrm{Na}\right]^{+}: 411.1527$; MP $152-154{ }^{\circ} \mathrm{C}$; Enantiometric excess was determined to be $77 \%$ ee via HPLC analysis using a Chiralpak IB column; solvent: 2-propanol/ hexane $=1 / 4$; flow rate: $1.0 \mathrm{~mL} / \mathrm{min}$; detection: at 254 $\mathrm{nm}): t_{\mathrm{R}}$ major $=17.72 \mathrm{~min}, t_{\mathrm{R}} \operatorname{minor}=23.90 \mathrm{~min} .[\alpha]_{589}{ }^{22.7^{\circ} \mathrm{C}}=-8.0(c=0.1, \mathrm{MeOH}$, for $77 \%$ ee sample).

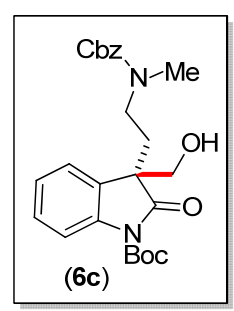

tert-Butyl (S)-3-(2-(((benzyloxy)carbonyl)(methyl)amino)ethyl)-3-(hydroxymethyl)2-oxoindoline-1-carboxylate (6c): $11 \mathrm{mg}$ (81\% yields) as colorless gel. $\mathrm{R}_{f}=0.22(40 \%$ EtOAc in hexane). ${ }^{1} \mathbf{H}$ NMR $\left(500 \mathrm{MHz}, \mathrm{CDCl}_{3}\right)$ (rotameric mixture) $\delta: 7.82(\mathrm{~d}, J=6.6$ $\mathrm{Hz}, 1 \mathrm{H}), 7.20-7.35$ (m, 7H), 7.05 (brs, 1H), 4.87-5.06 (m, 2H), 3.70-3.83 (m, 2H), 3.18 (brs, 0.5H), 2.96 (brs, 2.5H), 2.76 (s, 3H), 2.30 (brs, 1H), 2.08 (brs, 0.5H), 1.97-2.01 (m, 
0.5H), $1.63(\mathrm{~s}, 9 \mathrm{H}) ;{ }^{13} \mathrm{C}$ NMR $\left(125 \mathrm{MHz}, \mathrm{CDCl}_{3}\right.$ ) (rotameric mixture) $\delta: 177.5,177.2$, $171.3,156.0,155.9,148.9,140.2,140.0,136.7,136.6,128.7,128.4,127.9,127.7,124.8$, $124.7,123.2$, 122.9, 115.1, 84.7, 84.6, 68.1, 67.8, 67.1, 53.6, 45.0, 44.4, 34.5, 34.1, 31.3, 30.7, 28.1; IR (film) $v_{\max } 3445,2929,1730,1704,1480,1465,1405,1352,1293,1252$, 1216, 1151, $755 \mathrm{~cm}^{-1}$; HRMS (ESI) $\mathrm{m} / \mathrm{z} 477.1974[\mathrm{M}+\mathrm{Na}]^{+}$; calculated for $\left[\mathrm{C}_{25} \mathrm{H}_{30} \mathrm{~N}_{2} \mathrm{O}_{6}+\mathrm{Na}\right]^{+}: 477.1996$; Enantiometric excess was determined to be $90 \%$ ee via HPLC analysis using a Chiralpak IB column; solvent: 2-propanol/ hexane $=2 / 23$; flow rate: $1.0 \mathrm{~mL} / \mathrm{min}$; detection: at $254 \mathrm{~nm}$ ): $t_{\mathrm{R}}$ major $=16.68 \mathrm{~min}, t_{\mathrm{R}}$ minor $=20.67 \mathrm{~min}$. $[\alpha]_{589}{ }^{21.3^{\circ} \mathrm{C}}=-3.86(c=0.1125, \mathrm{MeOH}$, for $90 \%$ ee sample $)$.

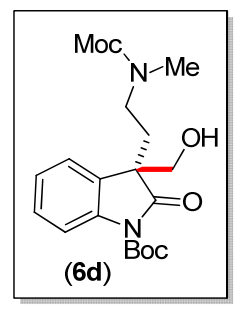

tert-Butyl (S)-3-(hydroxymethyl)-3-(2-((methoxycarbonyl)(methyl)amino)ethyl)-2oxoindoline-1-carboxylate (6d): $9.6 \mathrm{~g}$ (85\% yields) as white solid. $\mathrm{R}_{f}=0.15(40 \%$ EtOAc in hexane). ${ }^{1} \mathbf{H}$ NMR $\left(400 \mathrm{MHz}, \mathrm{CDCl}_{3}\right) \delta: 7.82$ (brs, $\left.1 \mathrm{H}\right), 7.17-7.33(\mathrm{~m}, 3 \mathrm{H})$, 3.76-3.83 (m, 2H), 3.45-3.56 (m, 3H), 2.87-3.11 (m, 2H), 2.70 (s, 3H), 2.33 (brs, 1H), 2.01-2.08 (m, 2H), $1.62(\mathrm{~s}, 9 \mathrm{H}) ;{ }^{13} \mathrm{C}$ NMR $\left(100 \mathrm{MHz}, \mathrm{CDCl}_{3}\right)$ (rotameric mixture) $\delta$ : $177.5,177.2$, 159.9, 156.73, 156.68, 148.9, 140.4, 140.1, 128.8, 128.2, 128.0, 124.74, 124.70, 123.0, 122.9, 122.8, 122.7, 115.2, 67.9, 67.8, 60.4, 53.4, 52.5, 44.3, 44.2, 34.1, 31.9, 31.2, 30.8, 29.7, 29.6, 28.1; IR (film) $v_{\max } 3435,2932,1763,1729,1703,1408$, 1465, 1252, 1151, $770 \mathrm{~cm}^{-1}$; HRMS (ESI) $\mathrm{m} / \mathrm{z} 401.1684[\mathrm{M}+\mathrm{Na}]^{+}$; calculated for $\left[\mathrm{C}_{19} \mathrm{H}_{26} \mathrm{~N}_{2} \mathrm{O}_{6}+\mathrm{Na}\right]^{+}: 401.1683$; Enantiometric excess was determined to be $86 \%$ ee via HPLC analysis using a Chiralpak IB column; solvent: 2-propanol/ hexane $=1 / 9$; flow rate: $1.0 \mathrm{~mL} / \mathrm{min}$; detection: at $254 \mathrm{~nm}$ ): $t_{\mathrm{R}}$ major $=11.83 \mathrm{~min}, t_{\mathrm{R}}$ minor $=14.30 \mathrm{~min}$. $[\alpha]_{589}{ }^{22}{ }^{\circ} \mathrm{C}=-16.0(c=0.05, \mathrm{MeOH}$, for $86 \%$ ee sample $)$. 


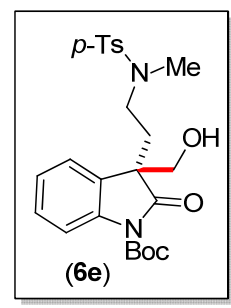

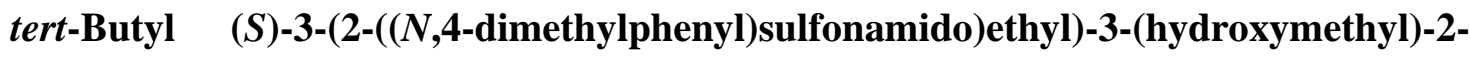
oxoindoline-1-carboxylate (6e): $12.5 \mathrm{~g}$ (88\% yields) as white solid. $\mathrm{R}_{f}=0.20(30 \%$ EtOAc in hexane). ${ }^{1} \mathbf{H}$ NMR $\left(400 \mathrm{MHz}, \mathrm{CDCl}_{3}\right) \delta: 7.82(\mathrm{~d}, J=8.1 \mathrm{~Hz}, 1 \mathrm{H}), 7.39-7.41$ (m, 2H), 7.29-7.33 (m, 1H), 7.15-7.2 (m, 4H), $3.73(\mathrm{ABq}, J=28.6,10.9 \mathrm{~Hz}, 2 \mathrm{H}), 2.77-$ $2.84(\mathrm{~m}, 1 \mathrm{H}), 2.52-2.59(\mathrm{~m}, 1 \mathrm{H}), 2.51(\mathrm{~s}, 3 \mathrm{H}), 2.33-2.38(\mathrm{~m}, 1 \mathrm{H}), 2.31(\mathrm{~s}, 3 \mathrm{H}), 1.96-1.99$ $(\mathrm{m}, 1 \mathrm{H}), 1.58(\mathrm{~s}, 9 \mathrm{H}) ;{ }^{13} \mathrm{C}$ NMR $\left(100 \mathrm{MHz}, \mathrm{CDCl}_{3}\right) \delta$ 177.6, 148.9, 143.3, 140.6, 133.9, 129.6, 129.0, 127.5, 127.4, 124.7, 122.8, 115.6, 84.6, 68.0, 53.2, 46.0, 34.7, 30.9, 28.1, 21.5; IR (film) $v_{\max } 3509,3056,2982,2932,1735,1461,1296,1251,1163,1091,1008$, $955 \mathrm{~cm}^{-1}$; HRMS (ESI) m/z 497.1720 $[\mathrm{M}+\mathrm{Na}]^{+}$; calculated for $\left[\mathrm{C}_{24} \mathrm{H}_{30} \mathrm{~N}_{2} \mathrm{O}_{6} \mathrm{~S}+\mathrm{Na}\right]^{+}$: 497.1717; MP 113-115 ${ }^{\circ} \mathrm{C}$; Enantiometric excess was determined to be $95 \%$ ee via HPLC analysis using a Chiralpak AD-H column; solvent: 2 -propanol/ hexane $=1 / 4$; flow rate: $1.0 \mathrm{~mL} / \mathrm{min}$; detection: at $254 \mathrm{~nm}$ ): $t_{\mathrm{R}}$ major $=18.64 \mathrm{~min}, t_{\mathrm{R}}$ minor $=10.98 \mathrm{~min}$. $[\alpha]_{589}{ }^{25.6^{\circ} \mathrm{C}}=+20.0(c=0.12, \mathrm{MeOH}$, for $95 \%$ ee sample $)$.

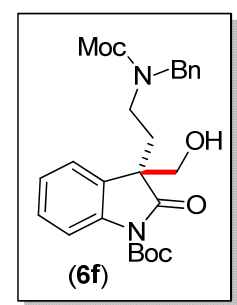

tert-Butyl (S)-3-(2-(benzyl(methoxycarbonyl)amino)ethyl)-3-(hydroxymethyl)-2oxoindoline-1-carboxylate (6f): $11 \mathrm{mg}$ (81\% yields) as colorless gel. $\mathrm{R}_{f}=0.19(30 \%$ EtOAc in hexane). ${ }^{1} \mathbf{H}$ NMR $\left(400 \mathrm{MHz}, \mathrm{CDCl}_{3}\right)$ (rotameric mixture) $\delta: 7.82(\mathrm{~s}, 0.5 \mathrm{H})$, $7.80(\mathrm{~s}, 0.5 \mathrm{H}), 7.09-7.33(\mathrm{~m}, 8 \mathrm{H}), 4.15-4.36(\mathrm{~m}, 2 \mathrm{H}), 3.71-3.80(\mathrm{~m}, 2 \mathrm{H}), 3.58-3.63(\mathrm{~m}$, 3H), 2.79-3.08 (m, 2H), 2.31-2.52 (m, 2H), 2.02-2.08 (m, 1H), $1.61(\mathrm{~s}, 9 \mathrm{H}) ;{ }^{13} \mathbf{C}$ NMR $\left(100 \mathrm{MHz}, \mathrm{CDCl}_{3}\right.$ ) (rotameric mixture) $\delta: 177.23,177.16,156.8,156.7,148.9,137.4$, $128.8,128.6,128.5,128.2,128.1,127.4,127.3,124.8,124.7,123.1,122.8,115.2$, 84.6, $84.5,68.1,67.8,53.5,52.8,50.1,50.0,42.7,41.5,31.4,30.7,29.69,29.66,28.08$; IR 
(film) $v_{\max } 3484,2982,2929,1733,1695,1465,1353,1392,1251,1151,1097,842,755$

$\mathrm{cm}^{-1}$; HRMS (ESI) m/z $477.1992[\mathrm{M}+\mathrm{Na}]^{+}$; calculated for $\left[\mathrm{C}_{25} \mathrm{H}_{30} \mathrm{~N}_{2} \mathrm{O}_{6}+\mathrm{Na}\right]^{+}$: 477.1996; Enantiometric excess was determined to be 91\% ee via HPLC analysis using a Chiralpak AD-H column; solvent: 2-propanol/ hexane $=1 / 9$; flow rate: $1.0 \mathrm{~mL} / \mathrm{min}$; detection: at $254 \mathrm{~nm}): t_{\mathrm{R}}$ major $=13.92 \mathrm{~min}, t_{\mathrm{R}}$ minor $=17.17 \mathrm{~min} .[\alpha]_{589}{ }^{21.6^{\circ} \mathrm{C}}=+4.0(\mathrm{c}=$ $0.1, \mathrm{MeOH}$, for $91 \%$ ee sample).

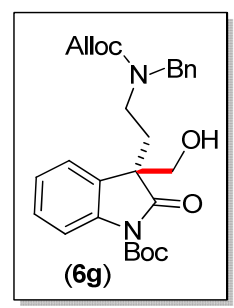

tert-Butyl (S)-3-(2-(((allyloxy)carbonyl)(benzyl)amino)ethyl)-3-(hydroxymethyl)-2oxoindoline-1-carboxylate (6g): $12.6 \mathrm{mg}$ (88\% yields) as white solid. $\mathrm{R}_{f}=0.30(30 \%$ EtOAc in hexane). ${ }^{1} \mathbf{H}$ NMR $\left(400 \mathrm{MHz}, \mathrm{CDCl}_{3}\right)$ (rotameric mixture) $\delta: 7.81(\mathrm{~s}, 0.5 \mathrm{H})$, $7.90(\mathrm{~s}, 0.5 \mathrm{H}), 7.10-7.29(\mathrm{~m}, 8 \mathrm{H}), 5.79-5.89(\mathrm{~m}, 1 \mathrm{H}), 5.17-5.22(\mathrm{~m}, 2 \mathrm{H}), 4.37-4.53(\mathrm{~m}$, 3H), 4.17-4.20 (m, 1H), 3.70-3.79 (m, 2H), 2.83-3.09 (m, 2H), 2.28-2.65 (m, 2H), 1.94 $(\mathrm{m}, 1 \mathrm{H}), 1.60(\mathrm{~s}, 9 \mathrm{H}) ;{ }^{13} \mathrm{C} \mathbf{N M R}\left(100 \mathrm{MHz}, \mathrm{CDCl}_{3}\right)$ (rotameric mixture) $\delta: 177.2,177.1$, $156.03,156.0,148.9,140.2,137.4,132.8,128.8,128.7,128.5,128.1,128.03,128.01$, $127.4,124.8,124.7,123.2,122.9,117.4,115.2,84.6,84.5,68.0,67.8,66.2,60.4,53.6$, 50.2, 42.8, 41.64, 41.61, 31.5, 30.8, 28.1, 21.0; IR (film) $v_{\max } 3470,2983,2937,1698$, 1465, 1291, 1251, 1151, 842, $755 \mathrm{~cm}^{-1}$; LRMS (ESI) m/z $479.2779[\mathrm{M}-\mathrm{H}]^{+}$; calculated for $\left[\mathrm{C}_{27} \mathrm{H}_{32} \mathrm{~N}_{2} \mathrm{O}_{6}-\mathrm{H}\right]^{+}:$479.2177; MP $60-62{ }^{\circ} \mathrm{C}$; Enantiometric excess was determined to be $92 \%$ ee via HPLC analysis using a Chiralpak AD-H column; solvent: 2-propanol/ hexane $=1 / 9$; flow rate: $1.0 \mathrm{~mL} / \mathrm{min}$; detection: at $254 \mathrm{~nm}$ ): $t_{\mathrm{R}}$ major $=13.19 \mathrm{~min}, t_{\mathrm{R}}$ minor $=16.52 \min .[\alpha]_{589}{ }^{21.3{ }^{\circ} \mathrm{C}}=-3.86(c=0.233, \mathrm{MeOH}$, for $92 \%$ ee sample $)$. 


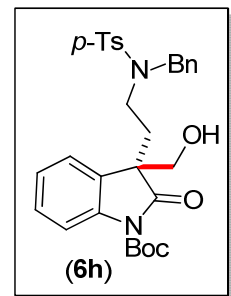

tert-Butyl
(S)-3-(2-((N-benzyl-4-methylphenyl)sulfonamido)ethyl)-3-

(hydroxymethyl)-2-oxoindoline-1-carboxylate (6h): $14.8 \mathrm{mg}(90 \%$ yields) as white solid. $\mathrm{R}_{f}=0.25\left(30 \%\right.$ EtOAc in hexane). ${ }^{1} \mathbf{H}$ NMR $\left(400 \mathrm{MHz}, \mathrm{CDCl}_{3}\right) \delta: 7.76(\mathrm{~d}, J=8.2$ $\mathrm{Hz}, 1 \mathrm{H}), 7.56(\mathrm{~d}, J=8.2 \mathrm{~Hz}, 2 \mathrm{H}), 7.24-7.31(\mathrm{~m}, 6 \mathrm{H}), 7.11-7.17(\mathrm{~m}, 3 \mathrm{H}), 6.99(\mathrm{~d}, J=7.0$ $\mathrm{Hz}, 1 \mathrm{H}), 4.21(\mathrm{ABq}, J=19.3,4.8 \mathrm{~Hz}, 2 \mathrm{H}), 3.66(\mathrm{ABq}, J=28.6 \mathrm{~Hz}, 2 \mathrm{H}), 2.65-2.82(\mathrm{~m}$, 2H), $2.40(\mathrm{~s}, 3 \mathrm{H}), 2.09-2.17(\mathrm{~m}, 1 \mathrm{H}), 1.82-1.89(\mathrm{~m}, 2 \mathrm{H}), 1.60(\mathrm{~s}, 9 \mathrm{H}) ;{ }^{13} \mathbf{C}$ NMR $(100$ $\left.\mathrm{MHz}, \mathrm{CDCl}_{3}\right) \delta 176.8,148.8,143.4,140.0,136.4,135.8,129.7,128.8,128.7,128.6$, $127.9,127.6,127.3,124.8,122.9,115.1,84.6,67.6,53.3,52.0,42.9,31.8,28.1,21.5$; IR (film) $v_{\max } 3475,2985,2932,1733,1654,1293,1159,939,755 \mathrm{~cm}^{-1}$; HRMS (ESI) m/z $573.2053[\mathrm{M}+\mathrm{Na}]^{+}$; calculated for $\left[\mathrm{C}_{30} \mathrm{H}_{34} \mathrm{~N}_{2} \mathrm{O}_{6} \mathrm{~S}+\mathrm{Na}\right]^{+}: 573.2030 ; \mathbf{M P} 150-152{ }^{\circ} \mathrm{C}$; Enantiometric excess was determined to be $97 \%$ ee via HPLC analysis using a Chiralpak AD-H column; solvent: 2-propanol/ hexane $=1 / 4$; flow rate: $1.0 \mathrm{~mL} / \mathrm{min}$; detection: at $254 \mathrm{~nm}): t_{\mathrm{R}}$ major $=38.62 \mathrm{~min}, t_{\mathrm{R}}$ minor $=46.62 \mathrm{~min} .[\alpha]_{589}{ }^{21.9^{\circ} \mathrm{C}}=-11.2(c=0.013$, $\mathrm{MeOH}$, for $97 \%$ ee sample).

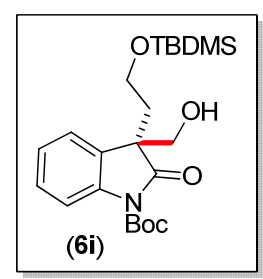

tert-Butyl

(S)-3-(2-((tert-butyldimethylsilyl)oxy)ethyl)-3-(hydroxymethyl)-2oxoindoline-1-carboxylate (6i): $11.2 \mathrm{mg}$ (89\% yields) as colorless gel. $\mathrm{R}_{f}=0.45(30 \%$ EtOAc in hexane). ${ }^{1} \mathbf{H}$ NMR $\left(500 \mathrm{MHz}, \mathrm{CDCl}_{3}\right) \delta: 7.88(\mathrm{~d}, J=8.2 \mathrm{~Hz}, 1 \mathrm{H}), 7.30-7.34$ (m, 1H), 7.24 (dd, $J=7.4,1.2 \mathrm{~Hz}, 1 \mathrm{H}), 7.18(\mathrm{td}, J=7.5,0.9 \mathrm{~Hz}, 1 \mathrm{H}), 3.78(\mathrm{~s}, 2 \mathrm{H}), 3.55-$ 3.59 (m, 1H), 4.6 (td, $J=10.1,4.0 \mathrm{~Hz}, 1 \mathrm{H}), 2.64$ (brs, 1H), 1.98 (t, $J=4.0 \mathrm{~Hz}, 1 \mathrm{H}), 1.96$ $(\mathrm{t}, J=4.0 \mathrm{~Hz}, 1 \mathrm{H}), 1.64(\mathrm{~s}, 9 \mathrm{H}), 0.76(\mathrm{~s}, 9 \mathrm{H}),-0.13(\mathrm{~s}, 3 \mathrm{H}),-0.14(\mathrm{~s}, 3 \mathrm{H}) ;{ }^{13} \mathrm{C}$ NMR $(125$ $\left.\mathrm{MHz}, \mathrm{CDCl}_{3}\right) \delta 177.6,149.3,140.3,128.7,128.4,124.2,123.3,115.2,84.1,68.3,59.0$, 
52.9, 35.8, 28.1, 25.8, 18.2, -5.85, -5.87; IR (film) $v_{\max } 3499,2932,2866,1772,1462$, 1296, 1253, 1152, 1134, $836 \mathrm{~cm}^{-1}$; HRMS (ESI) m/z $422.2336[\mathrm{M}+\mathrm{H}]^{+}$; calculated for $\left[\mathrm{C}_{22} \mathrm{H}_{35} \mathrm{NO}_{5} \mathrm{Si}+\mathrm{H}\right]^{+}:$422.2357; Enantiometric excess was determined to be $95 \%$ ee via HPLC analysis using a Chiralpak IC column; solvent: 2-propanol/ hexane $=1 / 19$; flow rate: $1.0 \mathrm{~mL} / \mathrm{min}$; detection: at $254 \mathrm{~nm}$ ): $t_{\mathrm{R}}$ major $=19.67 \mathrm{~min}, t_{\mathrm{R}}$ minor $=27.70 \mathrm{~min}$. $[\alpha]^{21.9}{ }_{589}=-48.0(c=0.1, \mathrm{MeOH}$, for $95 \%$ ee sample $)$.

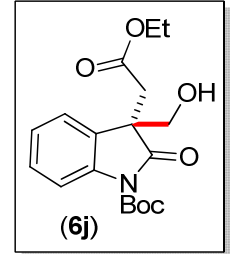

tert-Butyl

(S)-3-(2-ethoxy-2-oxoethyl)-3-(hydroxymethyl)-2-oxoindoline-1carboxylate (6j): $9.7 \mathrm{mg}$ (93\% yields) as white crystalline solid. $\mathrm{R}_{f}=0.15(30 \%$ EtOAc in hexane). ${ }^{1} \mathbf{H}$ NMR $\left(400 \mathrm{MHz}, \mathrm{CDCl}_{3}\right) \delta: 7.84(\mathrm{~d}, J=8.2 \mathrm{~Hz}, 1 \mathrm{H}), 7.30$ (t, $J=7.5 \mathrm{~Hz}$, $1 \mathrm{H}), 7.19(\mathrm{~d}, J=6.5 \mathrm{~Hz}, 1 \mathrm{H}), 7.13(\mathrm{t}, J=7.4 \mathrm{~Hz}, 1 \mathrm{H}), 3.80-3.89(\mathrm{~m}, 2 \mathrm{H}), 3.68-3.76(\mathrm{~m}$, 2H), $3.21(\mathrm{~d}, J=16.6 \mathrm{~Hz}, 1 \mathrm{H}), 2.88$ (d, $J=16.6 \mathrm{~Hz}, 1 \mathrm{H}), 2.47$ (brs, 1H), $1.62(\mathrm{~s}, 9 \mathrm{H})$, $0.97(\mathrm{t}, J=7.1 \mathrm{~Hz}, 3 \mathrm{H}) ;{ }^{13} \mathbf{C}$ NMR $\left(100 \mathrm{MHz}, \mathrm{CDCl}_{3}\right) \delta 171.9,164.2,143.8,135.2$, $123.6,122.7,119.2,117.3,109.9,79.2,62.1,55.5,46.6,32.9,22.8,8.4$; IR (film) $v_{\max }$ 3493, 2983, 2936, 1783, 1737, 1464, 1295, 1253, 1152, 1099, $756 \mathrm{~cm}^{-1}$; HRMS (ESI) $\mathrm{m} / \mathrm{z} 372.1411[\mathrm{M}+\mathrm{Na}]^{+}$; calculated for $\left[\mathrm{C}_{18} \mathrm{H}_{23} \mathrm{NO}_{6}+\mathrm{Na}\right]^{+}: 372.1418 ; \mathbf{M P} 132-134{ }^{\circ} \mathrm{C}$; Enantiometric excess was determined to be $91 \%$ ee via HPLC analysis using a Chiralpak IC3 column; solvent: 2-propanol/ hexane $=3 / 7$; flow rate: $1.0 \mathrm{~mL} / \mathrm{min}$; detection: at 254 $\mathrm{nm}): t_{\mathrm{R}}$ major $=19.76 \mathrm{~min}, t_{\mathrm{R}} \operatorname{minor}=32.92 \mathrm{~min} .[\alpha]^{21.7}{ }_{589}=+8.8(c=0.125, \mathrm{MeOH}$, for $91 \%$ ee sample).

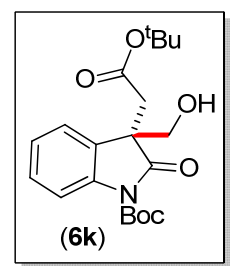

tert-Butyl (S)-3-(2-(tert-butoxy)-2-oxoethyl)-3-(hydroxymethyl)-2-oxoindoline-1carboxylate (6k): $10.7 \mathrm{mg}$ (95\% yields) as white solid. $\mathrm{R}_{f}=0.18(30 \%$ EtOAc in 
hexane). ${ }^{1} \mathbf{H}$ NMR $\left(400 \mathrm{MHz}, \mathrm{CDCl}_{3}\right) \delta: 7.85(\mathrm{~d}, J=8.2 \mathrm{~Hz}, 1 \mathrm{H}), 7.29(\mathrm{t}, J=7.4 \mathrm{~Hz}$, 1H), 7.19 (d, $J=6.7 \mathrm{~Hz}, 1 \mathrm{H}), 7.12$ (t, $J=7.4 \mathrm{~Hz}, 1 \mathrm{H}), 3.68$ (q, $J=11.2 \mathrm{~Hz}, 2 \mathrm{H}), 3.14$ (d, $J=15.9 \mathrm{~Hz}, 1 \mathrm{H}), 2.71$ (d, $J=15.9 \mathrm{~Hz}, 1 \mathrm{H}), 2.44$ (brs, 1H), 1.59 (s, 9H), 1.03 (s, 9H); ${ }^{13}$ C NMR (100 MHz, $\left.\mathrm{CDCl}_{3}\right) \delta 177.2,168.4,149.2,140.6,128.8,128.1,124.5,122.9$, 115.1, 84.4, 81.5, 67.6, 52.3, 39.6, 28.1, 27.3; IR (film) $v_{\max } 3461,2982,2935,1774$, 1734, 1462, 1294, 1253, 1153, 1098, 844, $756 \mathrm{~cm}^{-1}$; HRMS (ESI) m/z 400.1720 [M + $\mathrm{Na}]^{+}$; calculated for $\left[\mathrm{C}_{20} \mathrm{H}_{27} \mathrm{NO}_{6}+\mathrm{Na}\right]^{+}: 400.1731$; MP $88-90{ }^{\circ} \mathrm{C}$; Enantiometric excess was determined to be $93 \%$ ee via HPLC analysis using a Chiralpak IC column; solvent: 2-propanol/ hexane $=3 / 7$; flow rate: $2.0 \mathrm{~mL} / \mathrm{min}$; detection: at $254 \mathrm{~nm}$ ): $t_{\mathrm{R}}$ major $=4.87$ $\min , t_{\mathrm{R}}$ minor $=26.49 \min .[\alpha]^{20.8}{ }_{589}=-2.3(c=0.13, \mathrm{MeOH}$, for $93 \%$ ee sample $)$.

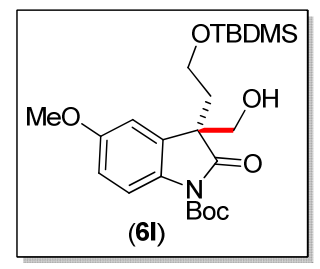

(S)-tert-Butyl

3-(2-((tert-butyldimethylsilyl)oxy)ethyl)-3-(hydroxymethyl)-5-

methoxy-2-oxoindoline-1-carboxylate (6I): $12 \mathrm{mg}$ (90\% yields) as colorless gel. $\mathrm{R}_{f}=$ $0.3\left(20 \%\right.$ EtOAc in hexane). ${ }^{1} \mathbf{H}$ NMR $\left(400 \mathrm{MHz}, \mathrm{CDCl}_{3}\right) \delta: 7.74(\mathrm{~d}, J=8.7 \mathrm{~Hz}, 1 \mathrm{H})$, 6.76-6.80 (m, 2H), 3.76 (s, 3H), 3.73 (brs, 2H), 3.49-3.54 (m, 1H), $3.44(\mathrm{td}, J=4.1,9.8$ $\mathrm{Hz}, 1 \mathrm{H}), 2.65$ (brs, 1H), 2.30-2.38 (m, 1H), 1.89 (dt, $J=4.0,14.2 \mathrm{~Hz}, 1 \mathrm{H}), 1.58(\mathrm{~s}, 9 \mathrm{H})$, $0.72(\mathrm{~s}, 9 \mathrm{H}),-0.16(\mathrm{~s}, 3 \mathrm{H}),-0.17(\mathrm{~s}, 3 \mathrm{H}) ;{ }^{13} \mathbf{C} \mathbf{N M R}\left(125 \mathrm{MHz}, \mathrm{CDCl}_{3}\right) \delta$ 177.6, 156.7, $149.3,133.7,130.2,116.0,112.8,109.9,83.9,68.2,59.0,55.6,53.3,35.9,28.1,25.8$, 18.2, -5.8, -5.9; IR (film) $v_{\max } 3483$ (br), 2977, 2896, 1770, 1727, 1598, 1485, 1369, 1341, 1302, 1250, 1155, 1125, 1072, 1044, 1005, $835 \mathrm{~cm}^{-1}$; HRMS (ESI) m/z 452.2462 $[\mathrm{M}+\mathrm{H}]^{+}$; calculated for $\left[\mathrm{C}_{23} \mathrm{H}_{37} \mathrm{NO}_{6} \mathrm{Si}+\mathrm{H}\right]^{+}:$452.2463; Enantiometric excess was determined to be $92 \%$ ee via HPLC analysis using a Chiralpak IC column; solvent: 2propanol $/$ hexane $=2 / 23$; flow rate: $1.0 \mathrm{~mL} / \mathrm{min}$; detection: at $254 \mathrm{~nm}$ ): $t_{\mathrm{R}}$ major $=14.26$ $\min , t_{\mathrm{R}} \operatorname{minor}=20.1 \mathrm{~min} .[\alpha]^{21.1}{ }_{589}=-25.86\left(c=0.116, \mathrm{CHCl}_{3}\right.$, for $91.6 \%$ ee sample $)$.

Formal Synthesis of (-)-physovenine (2e): 


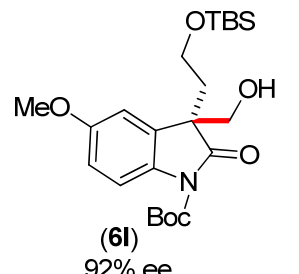

$92 \%$ ee

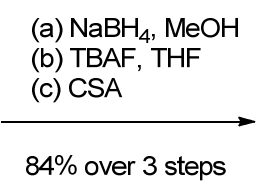

$84 \%$ over 3 steps

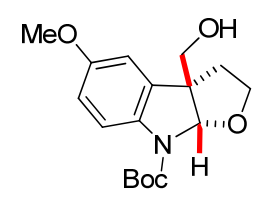

$(S, S)-9 a$

Step 1: In a solution of enantioenriched 61 (1 gm, $2.214 \mathrm{mmol} ; 1.0$ equiv $)$ in methanol (15 mL), $\mathrm{NaBH}_{4}\left(167 \mathrm{mg}, 4.43 \mathrm{mmol} ; 2.0\right.$ equiv) was added portionwise at $0{ }^{\circ} \mathrm{C}$ and stirred at $\mathrm{rt}$ for 30 minute. Upon completion of the reactions, the reaction mixture was quenched with satd. $\mathrm{NaHCO}_{3}$ solution and extracted with dichloromethane (2 X $\left.20 \mathrm{~mL}\right)$. The combined organic extracts were dried over anhydrous $\mathrm{Na}_{2} \mathrm{SO}_{4}$, and concentrated under reduced pressure. The crude products were directly treated for next step without any purification.

Step 2: In an oven-dried round-bottom flask, the crude material $(2.214 \mathrm{mmol} ; 1.0$ equiv. as prepared earlier) was taken in dry THF $(10 \mathrm{~mL})$ under argon atmosphere. To this reaction mixture tetrabutylammonium fluoride solution, $1.0 \mathrm{M}$ in THF $(2.4 \mathrm{~mL}, 2.4$ mmol; 1.1 equiv) was added dropwise and it was stirred for another $1 \mathrm{~h}$ at room temperature under argon atmosphere. Upon completion of the reactions, (TLC showed complete consumption of starting material) the reaction mixture was quenched with satd. $\mathrm{NH}_{4} \mathrm{Cl}$ solution and extracted with EtOAc $(2$ X $20 \mathrm{~mL})$. The crude products were purified by coloumn chromatography and afforded product.

Step 3: In an oven-dried round-bottom flask, compound (690 mg, $2.032 \mathrm{mmol} ; 1.0$ equiv. as prepared earlier step 2) was taken in dry THF $(15 \mathrm{~mL})$ under argon atmosphere. To this reaction mixture camphorsulfonic acid (4.7 gm, $20.33 \mathrm{mmol} ; 10.0$ equiv) was added and it was stirred for $2 \mathrm{~h}$ at room temperature under argon atmosphere. Upon completion of the reactions, the reaction mixture was quenched with $\mathrm{H}_{2} \mathrm{O}$ and extracted wit EtOAc (2 X $15 \mathrm{~mL}$ ). The organic filtrate was dried over $\mathrm{Na}_{2} \mathrm{SO}_{4}$ and concentrated in a rotary evaporator under vacuum. The crude products were purified by coloumn chromatography and afforded tricyclic alcohol 9a. 


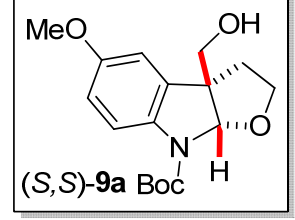

(3aS,8aS)-tert-Butyl

3a-(hydroxymethyl)-5-methoxy-3,3a-dihydro- $2 H$-furo[2,3-

b]indole-8(8aH)-carboxylate (9a): $548 \mathrm{mg}$ (84\% yields over 3 steps) as white solid. $\mathrm{R}_{f}=$ $0.3\left(50 \%\right.$ EtOAc in hexane). ${ }^{1} \mathbf{H}$ NMR $\left(400 \mathrm{MHz} \mathrm{CDCl}_{3}\right) \delta: 7.32-7.71(\mathrm{~m}, 1 \mathrm{H}), 6.73-$ $6.76(\mathrm{~m}, 2 \mathrm{H}), 5.89$ (brs, 1H), 3.97 (t, $J=7.9 \mathrm{~Hz}, 1 \mathrm{H}), 3.84-3.86(\mathrm{~m}, 1 \mathrm{H}), 3.75-3.77(\mathrm{~m}$, $1 \mathrm{H}), 3.75(\mathrm{~s}, 3 \mathrm{H}), 3.48-3.54(\mathrm{~m}, 1 \mathrm{H}), 2.18-2.20(\mathrm{~m}, 1 \mathrm{H}), 2.0-2.03(\mathrm{~m}, 1 \mathrm{H}), 1.54(\mathrm{~s}, 9 \mathrm{H})$; ${ }^{13} \mathrm{C}$ NMR $\left(100 \mathrm{MHz}, \mathrm{CDCl}_{3}\right) \delta: 155.9,152.5,115.1,113.4,109.9,96.1,81.2,67.2,66.4$, 60.4, 58.4, 55.7, 36.3, 28.4; IR (film) $v_{\max } 3460$ (br), 2977, 2875, 1704, 1682, 1492, 1393, 1369, 1276, 1257, 1164, 1150, 1079, 1040, $1024 \mathrm{~cm}^{-1}$; HRMS (ESI) m/z 344.1482 $[\mathrm{M}+\mathrm{Na}]^{+}$; calculated for $\left[\mathrm{C}_{17} \mathrm{H}_{23} \mathrm{NO}_{5}+\mathrm{Na}\right]^{+}: 344.1468 ;[\alpha]^{21.6}{ }_{589}=-7.6(c=0.25$, $\left.\mathrm{CHCl}_{3}\right)$.
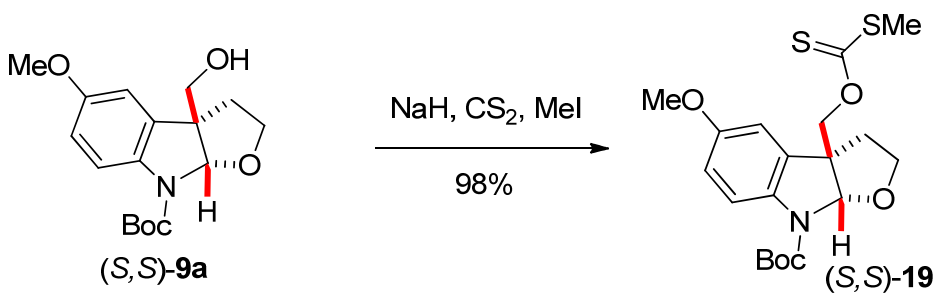

In an oven-dried round bottom flask, tricyclic primary alcohol 9a (563 mg, 1.751 mmol; 1.0 equiv) was taken in dry THF $(100 \mathrm{~mL})$. The flask was cooled to $0{ }^{\circ} \mathrm{C}$, and $\mathrm{NaH}$ (60 wt\%, $701 \mathrm{mg}, 17.517 \mathrm{mmol} ; 10.0$ equiv) was added in one portion. The suspension was stirred at $0{ }^{\circ} \mathrm{C}$ for $1 \mathrm{~h}$, and then $\mathrm{CS}_{2}(6.981 \mathrm{~mL}, 115.56 \mathrm{mmol} ; 66.0$ equiv) was added dropwise. The mixture was stirred at $0{ }^{\circ} \mathrm{C}$ for another $1 \mathrm{~h}$ then $\mathrm{MeI}(3.5 \mathrm{~mL}, 56.032$ mmol; 32.0 equiv) was added dropwise. The reaction mixture was stirred at $0{ }^{\circ} \mathrm{C}$ for another $1 \mathrm{~h}$, quenched dropwise with ice-water and then warm to room temperature. The mixture was diluted with brine $(30 \mathrm{~mL})$, extracted with ethyl acetate $(2 \times 150 \mathrm{~mL})$, dried over $\mathrm{MgSO}_{4}$ and concentrated under reduced pressure. The resultant crude was purified by flash chromatography to give xanthate 19 . 


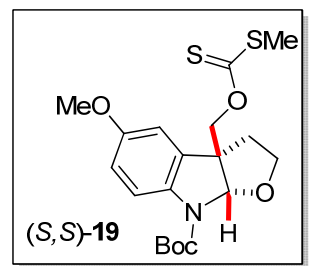

(3aS,8aS)-tert-Butyl 5-methoxy-3a-((((methylthio)carbonothioyl)oxy)methyl)-3,3adihydro-2H-furo[2,3-b]indole-8(8aH)-carboxylate (19): $710 \mathrm{mg}$ (98\% yields) as yellow gel. $\mathrm{R}_{f}=0.7$ (20\% EtOAc in hexane). ${ }^{1} \mathbf{H} \mathbf{~ N M R}\left(400 \mathrm{MHz}, \mathrm{CDCl}_{3}\right) \delta:$ 7.33-7.73 (m, 1H), 6.77-6.79 (m, 2H), $5.89(\mathrm{~s}, 1 \mathrm{H}), 4.71-4.78(\mathrm{~m}, 2 \mathrm{H}), 4.03(\mathrm{t}, J=7.8 \mathrm{~Hz}, 1 \mathrm{H})$, $3.77(\mathrm{~s}, 3 \mathrm{H}), 3.53-3.56(\mathrm{~m}, 1 \mathrm{H}), 2.43(\mathrm{~s}, 3 \mathrm{H}), 2.27-2.34(\mathrm{~m}, 1 \mathrm{H}), 2.17(\mathrm{dd}, J=4.5,12.0$ $\mathrm{Hz}, 1 \mathrm{H}), 1.57(\mathrm{~s}, 1 \mathrm{H}) ;{ }^{13} \mathrm{C}$ NMR $\left(100 \mathrm{MHz}, \mathrm{CDCl}_{3}\right) \delta: 215.2,156.0,152.1,136.8,131.7$, $115.3,114.0,110.0,95.9,75.6,67.3,55.7,53.4,36.7,29.7,28.4,18.7$; IR (film) $v_{\max }$ 2978, 2938, 2863, 1705, 1490, 1392, 1365, 1315, 1279, 1220, 1165, 1151, 1072, 1040, 973, 931, $858 \mathrm{~cm}^{-1}$; HRMS (ESI) m/z $434.1096[\mathrm{M}+\mathrm{Na}]^{+}$; calculated for $\left[\mathrm{C}_{19} \mathrm{H}_{25} \mathrm{NO}_{5} \mathrm{~S}_{2}\right.$ $+\mathrm{Na}]^{+}: 434.1066 ;[\alpha]^{21.5}{ }_{589}=-39.54\left(c=0.263, \mathrm{CHCl}_{3}\right)$.

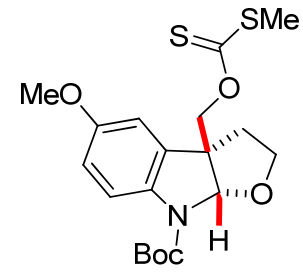

$(S, S)-19$

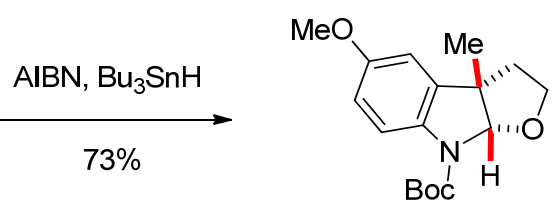

$(S, S)-9 b$

A solution of xanthate ester 19 (126 mg, $0.306 \mathrm{mmol} ; 1.0$ equiv) in $20 \mathrm{~mL}$ dry benzene was degassed for 15 min with Argon. The mixture was immersed in a preheated oil bath. When the solvent began to reflux, a mixture of tributylstannane (146 $\mu \mathrm{L}, 0.55$ mmol; 1.8 equiv) and AIBN (13 mg, $0.076 \mathrm{mmol} ; 0.25$ equiv) in $7 \mathrm{~mL}$ benzene was added slowly over $1.5 \mathrm{~h}$. The reaction was cooled after $2 \mathrm{~h}$ and evaporated. The residue was purified by chromatography on silica gel and afforded the cyclized compound $\mathbf{9 b}$. 


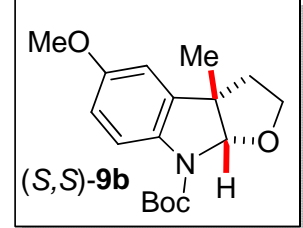

(3aS,8aS)-tert-Butyl

5-methoxy-3a-methyl-3,3a-dihydro-2H-furo[2,3-b]indole-

8(8aH)-carboxylate (9b): $68 \mathrm{mg}$ (73\% yields) as colorless gel. $\mathrm{R}_{f}=0.62(20 \%$ EtOAc in hexane). ${ }^{1} \mathbf{H}$ NMR (400 MHz, $\left.\mathrm{CDCl}_{3}\right) \delta: 7.32-7.71(\mathrm{~m}, 1 \mathrm{H}), 6.69-6.76(\mathrm{~m}, 1 \mathrm{H}), 5.63$ (s, $1 \mathrm{H}), 3.93-3.97(\mathrm{~m}, 1 \mathrm{H}), 3.77(\mathrm{~s}, 3 \mathrm{H}), 3.43-3.49(\mathrm{~m}, 1 \mathrm{H}), 2.07-2.17(\mathrm{~m}, 2 \mathrm{H}), 1.57(\mathrm{~s}, 9 \mathrm{H})$, 1.47 (s, 3H); ${ }^{13} \mathrm{C}$ NMR (100 MHz, $\left.\mathrm{CDCl}_{3}\right) \delta: 156.0,152.6,114.9,112.6,109.4,99.3$, 96.0, 81.0, 67.7, 55.7, 52.2, 41.3, 28.4, 25.0; IR (film) $v_{\max } 3030,2941,2853,1708$, 1481, 1395, 1369, 1277, 1169, 1084, 1044, 1029, 919, 862, $814 \mathrm{~cm}^{-1}$; HRMS (ESI) m/z $306.1687[\mathrm{M}+\mathrm{H}]^{+}$; calculated for $\left[\mathrm{C}_{17} \mathrm{H}_{23} \mathrm{NO}_{4}+\mathrm{H}\right]^{+}: 306.1700 ;[\alpha]^{21.1}{ }_{589}=-18.18(c=$ $\left.0.253, \mathrm{CHCl}_{3}\right)$.

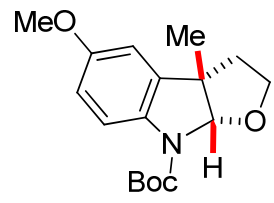

$(S, S)-9 b$

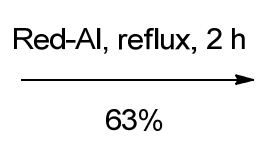

$63 \%$

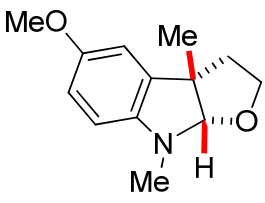

$(S, S)-9 c$

In a solution of compound $9 \mathbf{b}$ (45 $\mathrm{mg}, 0.147 \mathrm{mmol} ; 1.0$ equiv) in dry toluene (3 $\mathrm{mL})$, Red-Al (184 $\mu \mathrm{L}, 0.589 \mathrm{mmol} ; 4.0$ equiv) was added at $\mathrm{rt}$ and stirred it for 10 minute. Then the reaction mixture was heated to reflux for $2 \mathrm{~h}$. Upon completion of the reactions, the reaction mixture was quenched with potassium sodium tartrate solution and extracted with EtOAc $(2 \times 20 \mathrm{~mL})$. The combined organic extracts were dried over anhydrous $\mathrm{K}_{2} \mathrm{CO}_{3}$ and concentrated under reduced pressure. The crude products were purified by flash chromatography and afforded the product 9c.

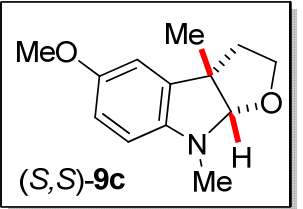


(3aS,8aS)-5-Methoxy-3a,8-dimethyl-3,3a,8,8a-tetrahydro-2H-furo[2,3-b]indole (9c): $20 \mathrm{mg}$ (63\% yields) as colorless gel. $\mathrm{R}_{f}=0.7$ (30\% EtOAc in hexane). ${ }^{1} \mathbf{H}$ NMR (400 $\left.\mathrm{MHz} \mathrm{CDCl}_{3}\right) \delta: 6.63-6.68(\mathrm{~m}, 1 \mathrm{H}), 6.27(\mathrm{~d}, J=8.3 \mathrm{~Hz}, 1 \mathrm{H}), 5.02(\mathrm{~s}, 1 \mathrm{H}), 3.91-3.95(\mathrm{~m}$, $1 \mathrm{H}), 3.74(\mathrm{~s}, 3 \mathrm{H}), 3.42-3.49(\mathrm{~m}, 1 \mathrm{H}), 2.87(\mathrm{~s}, 3 \mathrm{H}), 1.99-2.14(\mathrm{~m}, 2 \mathrm{H}), 1.43(\mathrm{~s}, 3 \mathrm{H}) ;{ }^{13} \mathrm{C}$ NMR $\left(100 \mathrm{MHz}, \mathrm{CDCl}_{3}\right) \delta: 152.7,145.0,136.0,112.2,110.5,105.6,105.3,67.4,56.1$, 52.5, 41.5, 31.7, 24.5; IR (film) $v_{\max } 2952,2863,2827,1605,1497,1281,1225,1214$, 1115, 1072, 1033, 1008, 913, 801, $704 \mathrm{~cm}^{-1}$; HRMS (ESI) m/z $220.1342[\mathrm{M} \mathrm{+} \mathrm{H}]^{+}$; calculated for $\left[\mathrm{C}_{13} \mathrm{H}_{17} \mathrm{NO}_{2}+\mathrm{H}\right]^{+}: 220.1332 ;[\alpha]_{589}{ }^{21.5}=-89.2\left(c=0.273, \mathrm{CHCl}_{3}\right)$. Lit. [(Matsuura, T.; Overman, L. E.; Poon, D. J. J. Am. Chem. Soc. 1998, 120, 6500) $[\alpha]_{\mathrm{D}}{ }^{25}=$ $\left.-100\left(c=0.4, \mathrm{CHCl}_{3}\right)\right]$.

Total Synthesis of $(+)$-coerulescine (1a):
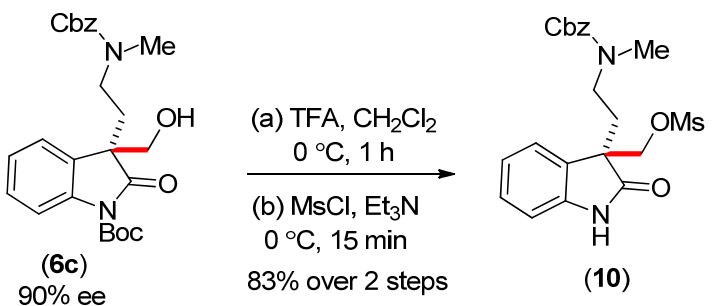

Step 1: In an oven-dried round-bottom flask, enantio-enriched compound 6c (49 mg, $0.108 \mathrm{mmol} ; 1.0$ equiv.) was taken in dichloromethane $(1 \mathrm{~mL})$. To this solution was added trifluoroacetic acid $(0.5 \mathrm{~mL})$ at $0{ }^{\circ} \mathrm{C}$ and was stirred for $1 \mathrm{~h}$ at room temperature. The reaction was quenched with saturated aqueous $\mathrm{NaHCO}_{3}$, and the organic phase was extracted with dichloromethane. The combined organic layers were washed with water and brine, and dried over anhydrous $\mathrm{Na}_{2} \mathrm{SO}_{4}$. After removal of the solvent, the residue was directly treated for next step without purification.

Step 2: The crude material $(0.108 \mathrm{mmol} ; 1.0$ equiv. as prepared earlier) was taken in dichloromethane $(2 \mathrm{~mL})$ under argon atmosphere and the reaction vessel was cooled to 0 ${ }^{\circ} \mathrm{C}$. To this reaction mixture triethylamine $(45 \mu \mathrm{L}, 0.324 \mathrm{mmol} ; 3.0$ equiv.) and methanesulfonylchloride (9 $\mu \mathrm{L}, 0.119 \mathrm{mmol} ; 1.1$ equiv.) was added and stirred it at $0{ }^{\circ} \mathrm{C}$. 
Upon completion of the reaction (monitoring by TLC), it was quenched with water and extracted with dichloromethane ( $2 \times 5 \mathrm{~mL})$. The combined organic extracts were dried over anhydrous $\mathrm{Na}_{2} \mathrm{SO}_{4}$, and concentrated under reduced pressure. The crude products were purified by flash chromatography to afford $\mathbf{1 0}$.

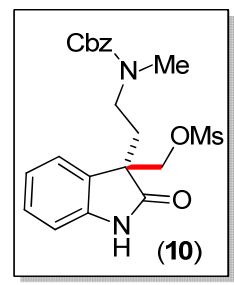

\section{(S)-(3-(2-(((Benzyloxy)carbonyl)(methyl)amino)ethyl)-2-oxoindolin-3-yl)methyl}

methanesulfonate (10): $39 \mathrm{mg}$ (83\% yields over 2 steps) as colorless gel. $\mathrm{R}_{f}=0.38(50 \%$ EtOAc in hexane). ${ }^{1} \mathbf{H}$ NMR (400 MHz, $\mathrm{CDCl}_{3}$ ) (rotameric mixture) $\delta: 8.53$ (brs, $0.5 \mathrm{H}$ ), 8.33 (brs, 0.5H), 7.2-7.36 (m, 7H), 6.96-7.07 (m, 1H), 6.85 (brs, 1H), 4.86-5.07 (m, 2H), 4.29-4.47 (m, 2H), 3.14-3.17 (m, 0.6H), 2.96-3.03 (m, 1.4H), 2.78 (brs, 6H), 2.14-2.19 $(\mathrm{m}, 1 \mathrm{H}), 1.84(\mathrm{brs}, 1 \mathrm{H}) ;{ }^{13} \mathbf{C}$ NMR $\left(100 \mathrm{MHz}, \mathrm{CDCl}_{3}\right.$ ) (rotameric mixture) $\delta: 177.8$, $177.7,155.9,141.2,129.2,128.5,128.0,127.9,127.9,123.8,123.0,110.3,72.5,67.2$, 67.1, 51.5, 51.4, 44.6, 44.2, 37.2, 34.7, 34.0, 30.7, 30.2; IR (film) $v_{\max } 3425,2979,2923$, 1685, 1369, 1166, 1020, $750 \mathrm{~cm}^{-1}$; HRMS (ESI) m/z 433.1450 [M + H] $]^{+}$; calculated for $\left[\mathrm{C}_{21} \mathrm{H}_{24} \mathrm{~N}_{2} \mathrm{O}_{6} \mathrm{~S}+\mathrm{H}\right]^{+}: 433.1428 ;[\alpha]^{20.8}{ }_{589}=+14\left(c=0.1, \mathrm{CHCl}_{3}\right)$.

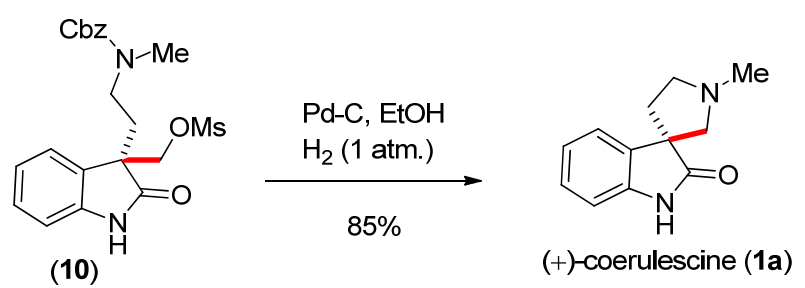

In an oven-dried round-bottom flask, the compound $10(0.042 \mathrm{mmol} ; 1.0$ equiv) was taken in ethanol $(2 \mathrm{~mL})$ under argon atmosphere. To this reaction mixture $\mathrm{Pd}$ on $\mathrm{C}$ ( $0.0042 \mathrm{mmol} ; 0.1$ equiv) was added portionwise and it was stirred for another $10 \mathrm{~min}$ at room temperature under argon atmosphere. Then the reaction mixture was stirred for $10 \mathrm{~h}$ under $\mathrm{H}_{2}$ (g) balloon. Upon completion of the reactions, (TLC showed complete 
consumption of starting material) the reaction mixture was filtered through celite and concentrated in a rotary evaporator under vacuum. The crude products were purified by coloumn chromatography and afforded (+)-coerulescine (1a).

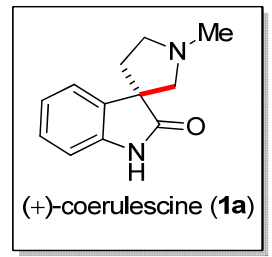

(S)-1'-Methylspiro[indoline-3,3'-pyrrolidin]-2-one (1a): $7.2 \mathrm{mg}$ (85\% yields) as colorless gel. $\mathrm{R}_{f}=0.1\left(5 \% \mathrm{MeOH}\right.$ in $\left.\mathrm{CH}_{2} \mathrm{Cl}_{2}\right) .{ }^{1} \mathbf{H}$ NMR $\left(400 \mathrm{MHz}, \mathrm{CDCl}_{3}\right) \delta: 9.04$ (brs, $1 \mathrm{H}), 7.36(\mathrm{~d}, J=7.3 \mathrm{~Hz}, 1 \mathrm{H}), 7.16(\mathrm{td}, J=1.0,7.6 \mathrm{~Hz}, 1 \mathrm{H}), 7.01(\mathrm{td}, J=0.8,6.8 \mathrm{~Hz}$, $1 \mathrm{H}), 6.88(\mathrm{~d}, J=7.7 \mathrm{~Hz}, 1 \mathrm{H}), 2.96-3.01(\mathrm{~m}, 1 \mathrm{H}), 2.89(\mathrm{~d}, J=9.4 \mathrm{~Hz}, 1 \mathrm{H}), 2.83(\mathrm{~d}, J=$ $9.4 \mathrm{~Hz}, 1 \mathrm{H}), 2.77-2.82(\mathrm{~m}, 1 \mathrm{H}), 2.44(\mathrm{~s}, 3 \mathrm{H}), 2.37-2.43(\mathrm{~m}, 1 \mathrm{H}), 2.05-2.12(\mathrm{~m}, 1 \mathrm{H}) ;{ }^{13} \mathrm{C}$ NMR $\left(100 \mathrm{MHz}, \mathrm{CDCl}_{3}\right) \delta: 183.3,140.3,136.2,127.8,123.2,122.8,109.7,66.4,56.8$, 53.8, 41.9, 38.0; IR (film) $v_{\max } 3430$ (br), 2932, 1654, 1192, 1050, $784 \mathrm{~cm}^{-1}$; HRMS (ESI) $\mathrm{m} / \mathrm{z} 203.1206[\mathrm{M}+\mathrm{H}]^{+}$; calculated for $\left[\mathrm{C}_{12} \mathrm{H}_{14} \mathrm{~N}_{2} \mathrm{O}+\mathrm{H}\right]^{+}: 203.1179 ;[\alpha]^{24.6}{ }_{589}=$ $+4.3\left(c=0.120, \mathrm{CHCl}_{3}\right)$; Lit. [Hayashi et al. Chem. Eur. J. 2014, 20, 13583] $\left[\alpha{ }^{28}{ }_{\mathrm{D}}=-\right.$ $1.1(c=1.2, \mathrm{MeOH})$.

\section{Total Synthesis of (+)-horsfiline (1b):}

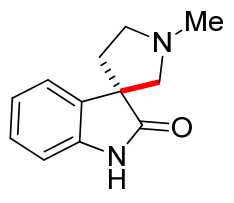

(+)-coerulescine (1a)

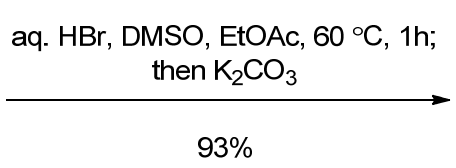

$93 \%$

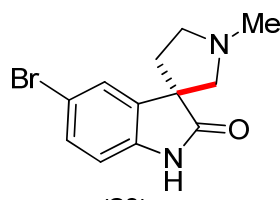

(20)

In a round bottom flask, (+)-coerulescine 1a (90 mg, $0.445 \mathrm{mmol} ; 1.0$ equiv) and DMSO (0.035 ml, $0.494 \mathrm{mmol} ; 1.1$ equiv) was dissolved in ethylacetate $(6 \mathrm{~mL})$. Then aqueous hydrobromic acid ( $60 \mathrm{wt} \%, 250 \mu \mathrm{L}, 1.481 \mathrm{mmol} ; 3.3$ equiv) was added to the solution at $60{ }^{\circ} \mathrm{C}$ for $1 \mathrm{~h}$ under air and then at same temperature $\mathrm{K}_{2} \mathrm{CO}_{3}$ (90 mg) was added and the reaction mixture was stirred for another $1 \mathrm{~h}$. After cooling down to room 
temperature and concentrating in vacuum, the resultant crude was purified by flash chromatography on neutral alumina to give (-)-Br-coerulescine 20.

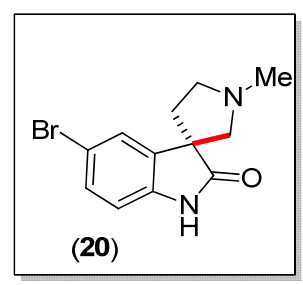

(S)-5-Bromo-1'-methylspiro[indoline-3,3'-pyrrolidin]-2-one (20): $116 \mathrm{mg} \quad$ (93\% yields) as brown solid. $\mathrm{R}_{f}=0.2\left(\right.$ EtOAc in one drop $\left.\mathrm{Et}_{3} \mathrm{~N}\right) .{ }^{\mathbf{1}} \mathbf{H} \mathbf{~ N M R}\left(400 \mathrm{MHz}, \mathrm{CDCl}_{3}\right)$ $\delta: 8.76(\mathrm{brs}, 1 \mathrm{H}), 7.52(\mathrm{~d}, J=1.8 \mathrm{~Hz}, 1 \mathrm{H}), 7.29(\mathrm{dd}, J=1.9,8.2 \mathrm{~Hz}, 1 \mathrm{H}), 6.76(\mathrm{~d}, J=8.2$ $\mathrm{Hz}, 1 \mathrm{H}), 3.03-2.98(\mathrm{~m}, 1 \mathrm{H}), 2.81(\mathrm{~s}, 2 \mathrm{H}), 2.70(\mathrm{q}, J=7.9 \mathrm{~Hz}, 1 \mathrm{H}), 2.42(\mathrm{~s}, 3 \mathrm{H}), 2.41-$ $2.35(\mathrm{~m}, 1 \mathrm{H}), 2.05(\mathrm{td}, J=7.4,13.0 \mathrm{~Hz}, 1 \mathrm{H}) ;{ }^{13} \mathbf{C ~ N M R}\left(100 \mathrm{MHz}, \mathrm{CDCl}_{3}\right) \delta: 182.3$, 139.1, 138.5, 130.6, 126.6, 115.4, 111.0, 66.3, 56.5, 53.9, 41.7, 37.8; IR (film) $v_{\max } 3410$, 3221, 2980, 1688, 1618, 1458, 1319, $1169 \mathrm{~cm}^{-1}$; HRMS (ESI) m/z $281.0298[\mathrm{M}+\mathrm{H}]^{+}$; calculated for $\left[\mathrm{C}_{12} \mathrm{H}_{13} \mathrm{BrN}_{2} \mathrm{O}+\mathrm{H}\right]^{+}: 281.0284 ; \mathrm{MP}=142{ }^{\circ} \mathrm{C} ;[\alpha]^{24.8}{ }_{589}=+15.0(\mathrm{c}=$ $\left.0.232, \mathrm{CHCl}_{3}\right)$.

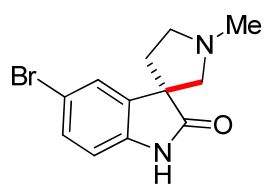

(20)

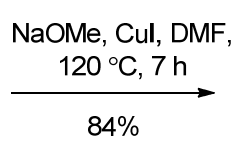

In a suspension of compound 20 (36 mg, $0.128 \mathrm{mmol} ; 1.0$ equiv) and $\mathrm{CuI}$ (49 $\mathrm{mg}, 0.256 \mathrm{mmol} ; 2.0$ equiv) in $\operatorname{DMF}(0.5 \mathrm{ml})$ was added sodium methoxide solution prepared from a lump of sodium ( $88 \mathrm{mg}, 3.84 \mathrm{mmol} ; 30.0$ equiv) and absolute $\mathrm{MeOH}$ (4 $\mathrm{mL}$ ). After the resulting mixture was stirred at $120{ }^{\circ} \mathrm{C}$ for $7 \mathrm{~h}$, the reaction mixture was cooled and the insoluble materials were filtered off. The filtrate was concentrated in vacuum and $\mathrm{H}_{2} \mathrm{O}$ was added to the residue. The aqueous layer extracted with $\mathrm{CH}_{2} \mathrm{Cl}_{2}$ (3 $\mathrm{X} 5 \mathrm{~mL}$ ) and the extract was washed with brine and dried over anhydrous $\mathrm{K}_{2} \mathrm{CO}_{3}$ and concentrated under reduced pressure. The crude products were purified by flash chromatography on neutral alumina and afforded the product (+)-horsfiline $\mathbf{1 b}$. 


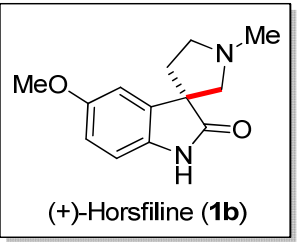

(S)-5-Methoxy-1'-methylspiro[indoline-3,3'-pyrrolidin]-2-one (1b): $25 \mathrm{mg}$ (84\%

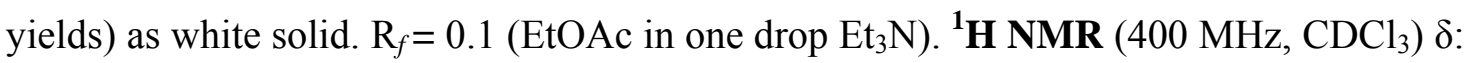
8.88 (brs, 1H), 7.02 (d, $J=2.4 \mathrm{~Hz}, 1 \mathrm{H}), 6.78$ (d, $J=8.4 \mathrm{~Hz}, 1 \mathrm{H}), 6.69$ (dd, $J=2.5,8.4$ Hz, 1H), 3.76 (s, 3H), 3.04-2.99 (m, 1H), 2.87 (s, 2H), 2.83-2.75 (m, 1H), 2.45 (s, 3H),

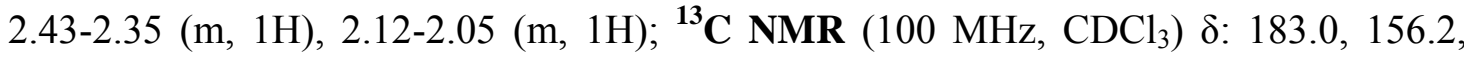
137.4, 133.6, 112.6, 110.3, 110.0, 66.2, 56.7, 55.9, 54.2, 41.8, 38.0; IR (film) $v_{\max } 3423$, 2960, 2837, 1500, 1489, 1207, 1030, $970 \mathrm{~cm}^{-1}$; HRMS (ESI) m/z 233.1306 [M + H] ; calculated for $\left[\mathrm{C}_{13} \mathrm{H}_{16} \mathrm{~N}_{2} \mathrm{O}_{2}+\mathrm{H}\right]^{+}: 233.1285 ; \mathrm{MP}=153{ }^{\circ} \mathrm{C} ;[\alpha]^{24.9}{ }_{589}=+7.5(c=0.144$, $\left.\mathrm{CHCl}_{3}\right)$; Lit. [Hayashi et al. Chem. Eur. J. 2014, 20, 13583] $[\alpha]{ }_{\mathrm{D}}^{25}=-7.0(c=0.30$, $\mathrm{MeOH})]$.

\section{Total Synthesis of (-)-deoxyeseroline (2c) and (-)-esermethole (2a):}
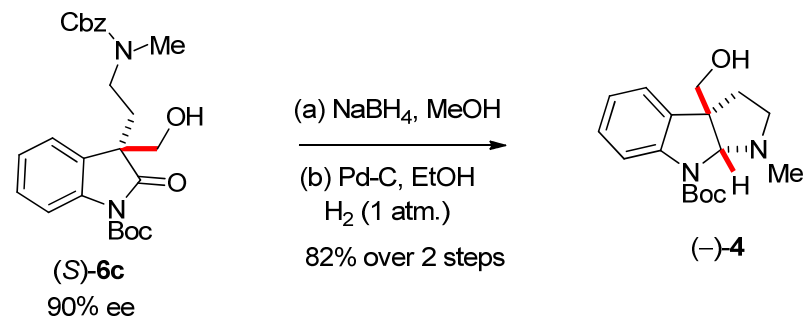

Step 1: In a solution of oxindole 6c $(320 \mathrm{mg}, 0.705 \mathrm{mmol} ; 1.0$ equiv) in methanol (10 $\mathrm{mL}), \mathrm{NaBH}_{4}\left(67 \mathrm{mg}, 1.76 \mathrm{mmol} ; 2.5\right.$ equiv) was added portionwise at $0{ }^{\circ} \mathrm{C}$ and stirred at rt for 30 minute. Upon completion of the reactions, the reaction mixture was quenched with satd. $\mathrm{NaHCO}_{3}$ solution and extracted with dichloromethane $(2 \mathrm{X} 10 \mathrm{~mL})$. The combined organic extracts were dried over anhydrous $\mathrm{Na}_{2} \mathrm{SO}_{4}$, and concentrated under reduced pressure. The crude products were directly treated for next step without any purification. 
Step 2: In an oven-dried round-bottom flask, the crude material $(0.705 \mathrm{mmol} ; 1.0$ equiv. as prepared earlier) was taken in ethanol $(20 \mathrm{~mL})$ under argon atmosphere. To this reaction mixture $\mathrm{Pd}$ on $\mathrm{C}(50 \mathrm{mg})$ was added portionwise and it was stirred for another 10 min at room temperature under argon atmosphere. Then the reaction mixture was stirred for $10 \mathrm{~h}$ under $\mathrm{H}_{2}$ (g) balloon. Upon completion of the reactions, (TLC showed complete consumption of starting material) the reaction mixture was filtered through Celite and concentrated in a rotary evaporator under vacuum. The crude products were purified by coloumn chromatography and afforded tricyclic product (-)-4.

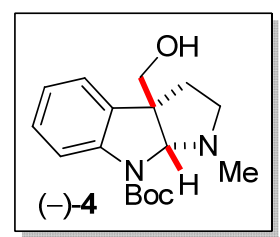

(3aR,8aR)-tert-Butyl 3a-(hydroxymethyl)-1-methyl-1,3,3a,8a-tetrahydropyrrolo[2,3b]indole-8(2H)-carboxylate (4): $176 \mathrm{mg}$ (82\% yields over 2 steps) as colorless gel. $\mathrm{R}_{f}=$ $0.2\left(50 \%\right.$ EtOAc in hexane). ${ }^{1} \mathbf{H}$ NMR (400 MHz, $\left.\mathrm{CDCl}_{3}\right) \delta: 7.63$ (brs, 1H), 7.19-7.15 (m, 1H), $7.08(\mathrm{~d}, J=7.3 \mathrm{~Hz}, 1 \mathrm{H}), 6.97(\mathrm{t}, J=7.4 \mathrm{~Hz}, 1 \mathrm{H}), 5.0(\mathrm{~s}, 1 \mathrm{H}), 3.63$ (q, $J=11.0$ $\mathrm{Hz}, 2 \mathrm{H}), 2.74$ (brs, 1H), 2.64-2.61 (m, 2H), 2.53 (s, 3H), 2.17-2.10 (m, 1H), 1.91-1.86 $(\mathrm{m}, 1 \mathrm{H}), 1.54(\mathrm{~s}, 9 \mathrm{H}) ;{ }^{13} \mathrm{C}$ NMR $\left(100 \mathrm{MHz}, \mathrm{CDCl}_{3}\right) \delta: 153.3,142.9,134.8,128.3,123.3$, 123.2, 116.2, 85.5, 81.5, 67.0, 58.2, 53.3, 38.5, 33.8, 28.4; IR (film) $v_{\max } 3371$ (br), 2930, 2856, 1703, 1480, 1388, 1369, 1313, 1254, 1168, 1156, 1076, 1031, 1018, $756 \mathrm{~cm}^{-1}$; HRMS (ESI) m/z $305.1882[\mathrm{M}+\mathrm{H}]^{+}$; calculated for $\left[\mathrm{C}_{17} \mathrm{H}_{24} \mathrm{~N}_{2} \mathrm{O}_{3}+\mathrm{H}\right]^{+}$: 305.1860; $[\alpha]$ $22.4{ }_{589}=-2.83\left(c=0.247, \mathrm{CHCl}_{3}\right)$.

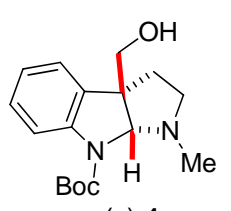

$(-)-4$

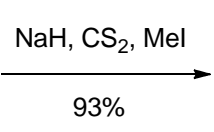

$3 \%$

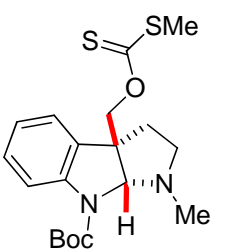

$(-)-21$

In an oven-dried round bottom flask, tricyclic alcohol 4 (150 mg, $0.493 \mathrm{mmol} ; 1.0$ equiv) was taken in dry THF $(50 \mathrm{~mL})$. The flask was cooled to $0{ }^{\circ} \mathrm{C}$, and $\mathrm{NaH}(60 \mathrm{wt} \%, 197 \mathrm{mg}$, 
$4.93 \mathrm{mmol} ; 10.0$ equiv) was added in one portion. The suspension was stirred at $0{ }^{\circ} \mathrm{C}$ for $1 \mathrm{~h}$, and then $\mathrm{CS}_{2}(1.97 \mathrm{~mL}, 32.538 \mathrm{mmol} ; 66.0$ equiv) was added dropwise. The mixture was stirred at $0{ }^{\circ} \mathrm{C}$ for another $1 \mathrm{~h}$ then MeI $(986 \mu \mathrm{L}, 15.776 \mathrm{mmol} ; 32.0$ equiv) was added dropwise. The reaction mixture was stirred at $0{ }^{\circ} \mathrm{C}$ for another $1 \mathrm{~h}$, quenched dropwise with ice-water and then warm to room temperature. The mixture was diluted with brine $(10 \mathrm{~mL})$, extracted with ethyl acetate $(2 \mathrm{X} 100 \mathrm{~mL})$, dried over $\mathrm{MgSO}_{4}$ and concentrated under reduced pressure. The resultant crude was purified by flash chromatography to give xanthate 21 .

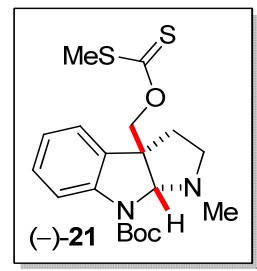

$(3 \mathrm{a}, 8 \mathrm{a} R)$-tert-Butyl

1-methyl-3a-((((methylthio)carbonothioyl)oxy)methyl)1,3,3a,8a-tetrahydropyrrolo[2,3-b]indole-8(2H)-carboxylate (21): $181 \mathrm{mg}$ (93\% yields) as yellow gel. $\mathrm{R}_{f}=0.45$ (40\% EtOAc in hexane). ${ }^{1} \mathbf{H}$ NMR $\left(500 \mathrm{MHz}, \mathrm{CDCl}_{3}\right) \delta$ : 7.69 (brs, 1H), 7.25 (t, $J=7.8 \mathrm{~Hz}, 1 \mathrm{H}), 7.19$ (d, $J=7.5 \mathrm{~Hz}, 1 \mathrm{H}), 7.04$ (t, $J=7.5 \mathrm{~Hz}, 1 \mathrm{H})$, $5.06(\mathrm{~s}, 1 \mathrm{H}), 4.68-4.74(\mathrm{~m}, 2 \mathrm{H}), 2.69-2.77(\mathrm{~m}, 2 \mathrm{H}), 2.60(\mathrm{~s}, 3 \mathrm{H}), 2.43(\mathrm{~s}, 3 \mathrm{H}), 2.23-2.28$ (m, 1H), 2.07-2.12 (m, 1H), $1.60(\mathrm{~s}, 9 \mathrm{H}) ;{ }^{13} \mathrm{C}$ NMR $\left(125 \mathrm{MHz}, \mathrm{CDCl}_{3}\right) \delta: 215.3,153.0$, $128.7,123.5,123.3,123.2,116.3,85.8,81.6,76.7,53.1,38.6,34.3,28.41,28.39,27.1$, 18.6; IR (film) $v_{\max } 2974,2856,2794,1711,1697,1602,1469,1387,1215,1151,1079$, $1067,1024 \mathrm{~cm}^{-1}$; HRMS (ESI) $\mathrm{m} / \mathrm{z} 395.1464[\mathrm{M}+\mathrm{H}]^{+}$; calculated for $\left[\mathrm{C}_{19} \mathrm{H}_{26} \mathrm{~N}_{2} \mathrm{O}_{3} \mathrm{~S}_{2}+\right.$ $\mathrm{H}]^{+}: 395.1458 ;[\alpha]^{20.4}{ }_{589}=-60.57\left(c=0.317, \mathrm{CHCl}_{3}\right)$.
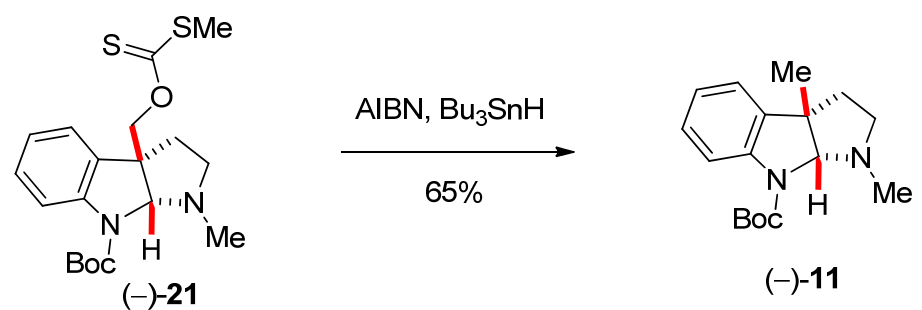
A solution of xanthate ester 21 (100 mg, $0.253 \mathrm{mmol}$; 1.0 equiv) in $15 \mathrm{~mL}$ dry benzene was degassed for 15 min with Argon. The mixture was immersed in a preheated oilbath. When the solvent began to reflux, a mixture of tributylstannane $(121 \mu \mathrm{L}, 0.455 \mathrm{mmol}$; 1.8 equiv) and AIBN (10 mg, $0.063 \mathrm{mmol} ; 0.25$ equiv) in $5 \mathrm{~mL}$ benzene was added slowly over $1.5 \mathrm{~h}$. The reaction was cooled after $2 \mathrm{~h}$ and evaporated. The residue was purified by chromatography on silica gel and afforded the cyclized compound $\mathbf{1 1}$.

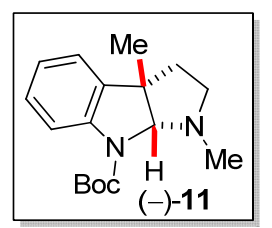

(3aS,8aR)-tert-Butyl 1,3a-dimethyl-1,3,3a,8a-tetrahydropyrrolo[2,3-b]indole-8(2H)carboxylate (11): $47 \mathrm{mg}$ (65\% yields) as colorless gel. $\mathrm{R}_{f}=0.5(20 \%$ EtOAc in hexane). ${ }^{1}$ H NMR (400 MHz, $\left.\mathrm{CDCl}_{3}\right) \delta: 7.62$ (brs, 1H), 7.13-7.17 (m, 1H), 7.09 (d, $J=6.8 \mathrm{~Hz}$, $1 \mathrm{H}), 6.98(\mathrm{td}, J=0.6,7.4 \mathrm{~Hz}, 1 \mathrm{H}), 4.82(\mathrm{~s}, 1 \mathrm{H}), 2.58-2.66(\mathrm{~m}, 2 \mathrm{H}), 2.55(\mathrm{~s}, 3 \mathrm{H}), 1.99-$ 2.03 (m, 2H), 1.57 (s, 9H), 1.41 (s, 3H); $\left.{ }^{13} \mathbf{C ~ N M R ~ ( 1 0 0 ~ M H z , ~} \mathrm{CDCl}_{3}\right)$ 8: 153.4, 141.7, 139.1, 127.6, 123.2, 122.7, 115.9, 89.1, 81.3, 53.4, 52.1, 39.1, 38.3, 28.4, 26.4; IR (film) $v_{\max } 3430$ (br), 3015, 2993, 2840, 1732, 1698, 1682, 1593, 1482, 1390, 1364, 1246, 1020, 934, 757, $667 \mathrm{~cm}^{-1}$; HRMS (ESI) m/z 289.1925 [M+ H] $]^{+}$; calculated for $\left[\mathrm{C}_{17} \mathrm{H}_{24} \mathrm{~N}_{2} \mathrm{O}_{2}+\right.$ $\mathrm{H}]^{+}: 289.1911 ;[\alpha]^{20.5}{ }_{589}=-10.15\left(c=0.197, \mathrm{CHCl}_{3}\right)$.

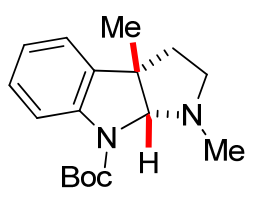

$(-)-11$

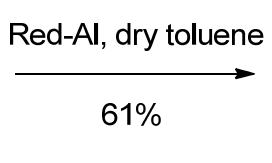

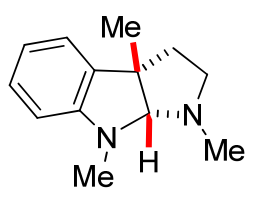

deoxyeseroline (2c) 
In a solution of compound 11 (40 mg, $0.139 \mathrm{mmol} ; 1.0$ equiv) in dry toluene (2 $\mathrm{mL})$, Red-Al (302 $\mu \mathrm{L}, 0.97 \mathrm{mmol} ; 7.0$ equiv) was added at rt and stirred it for 10 minute. Then the reaction mixture was heated to reflux for $2 \mathrm{~h}$. Upon completion of the reactions, the reaction mixture was quenched with Potassium sodium tartrate solution and extracted with EtOAc $(2 \times 10 \mathrm{~mL})$. The combined organic extracts were dried over anhydrous $\mathrm{K}_{2} \mathrm{CO}_{3}$ and concentrated under reduced pressure. The crude products were purified by flash chromatography and afforded the product (-)-deoxyeseroline (2c).

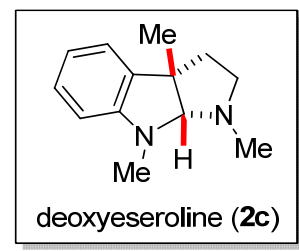

(3aS,8aR)-1,3a,8-Trimethyl-1,2,3,3a,8,8a-hexahydropyrrolo[2,3-b]indole (2c): $17 \mathrm{mg}$

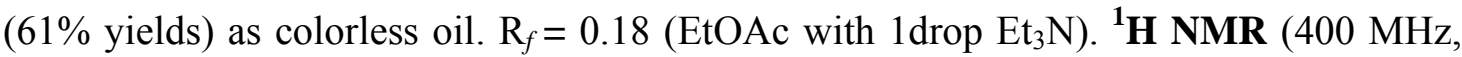
$\left.\mathrm{CDCl}_{3}\right) \delta: 7.06(\mathrm{td}, J=1.0 \mathrm{~Hz}, 7.8 \mathrm{~Hz}, 1 \mathrm{H}), 6.98(\mathrm{~d}, J=7.2 \mathrm{~Hz}, 1 \mathrm{H}), 6.65(\mathrm{t}, J=7.4 \mathrm{~Hz}$, $1 \mathrm{H}), 6.40(\mathrm{~d}, J=7.8 \mathrm{~Hz}, 1 \mathrm{H}), 4.09(\mathrm{~s}, 1 \mathrm{H}), 2.93(\mathrm{~s}, 3 \mathrm{H}), 2.68-2.73(\mathrm{~m}, 1 \mathrm{H}), 2.59-2.65(\mathrm{~m}$, $1 \mathrm{H}), 2.54(\mathrm{~s}, 3 \mathrm{H}), 1.93-1.96(\mathrm{~m}, 2 \mathrm{H}), 1.42(\mathrm{~s}, 3 \mathrm{H}) ;{ }^{13} \mathrm{C}$ NMR $\left(100 \mathrm{MHz}, \mathrm{CDCl}_{3}\right) \delta$ : 152.0, 136.7, 127.7, 122.2, 117.5, 106.6, 97.6, 53.3, 52.7, 40.9, 38.5, 36.5, 27.4; IR (film) $v_{\max } 3055,3021,2890,2841,1605,1598,1580,1486,1447,1371,1348,1300,1254$, 1172, 1123, 1035, $956 \mathrm{~cm}^{-1}$; HRMS (ESI) m/z $203.1562[\mathrm{M}+\mathrm{H}]^{+}$; calculated for $\left[\mathrm{C}_{13} \mathrm{H}_{18} \mathrm{~N}_{2}+\mathrm{H}\right]^{+}:$203.1543; $[\alpha]_{589}^{20.7}=-68\left(c=0.233, \mathrm{CHCl}_{3}\right)$; Lit. [Lim, H. J.; RajanBabu, T. V. Org. Lett. 2000, 2, 675] $[\alpha]^{25}{ }_{\mathrm{D}}=-71\left(c=0.0185, \mathrm{CHCl}_{3}\right)$.
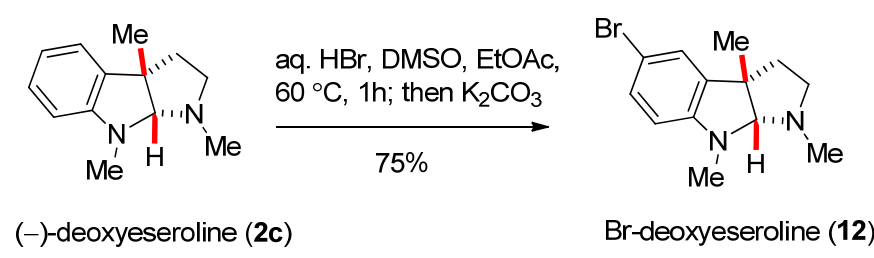

In a round bottom flask, (-)-deoxyeseroline 2c (100 mg, $0.494 \mathrm{mmol} ; 1.0$ equiv) and DMSO (0.039 ml, $0.549 \mathrm{mmol} ; 1.1$ equiv) was dissolved in ethylacetate $(6 \mathrm{~mL})$. Then aqueous hydrobromic acid ( $60 \mathrm{wt} \%, 278 \mu \mathrm{L}, 1.646 \mathrm{mmol} ; 3.3$ equiv) was added to 
the solution at $60{ }^{\circ} \mathrm{C}$ for $1 \mathrm{~h}$ under air and then at same temperature $\mathrm{K}_{2} \mathrm{CO}_{3}(100 \mathrm{mg})$ was added and the reaction mixture was stirred for another $1 \mathrm{~h}$. After cooling down to room temperature and concentrating in vacuum, the resultant crude was purified by flash chromatography on neutral alumina to give 12.

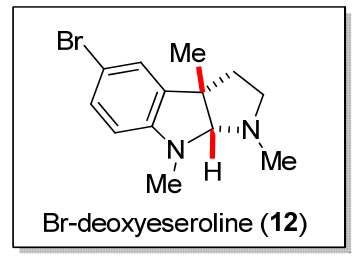

(3aS,8aR)-5-Bromo-1,3a,8-trimethyl-1,2,3,3a,8,8a-hexahydropyrrolo[2,3-b]indole (12): $104 \mathrm{mg}$ (75\% yields) as yellow oil. $\mathrm{R}_{f}=0.1\left(5 \% \mathrm{MeOH}\right.$ in $\left.\mathrm{CH}_{2} \mathrm{Cl}_{2}\right) .{ }^{1} \mathbf{H}$ NMR (400 $\left.\mathrm{MHz} \mathrm{CDCl}_{3}\right) \delta: 7.13(\mathrm{dd}, J=2.0,8.3 \mathrm{~Hz}, 1 \mathrm{H}), 7.03(\mathrm{~d}, J=2.0 \mathrm{~Hz}, 1 \mathrm{H}), 6.23(\mathrm{~d}, J=8.3$ $\mathrm{Hz}, 1 \mathrm{H}), 4.07$ (s, 1H), 2.89 (s, 3H), 2.72-2.58 (m, 2H), 2.52 (s, 3H), 1.94-1.91 (m, 2H), 1.39 (s, 3H); ${ }^{13} \mathrm{C}$ NMR $\left(100 \mathrm{MHz}, \mathrm{CDCl}_{3}\right) \delta: 150.8,139.0,130.3,125.4,108.9,107.8$, 97.6, 53.2, 52.7, 40.6, 38.7, 36.2, 27.2; IR (film) $v_{\max } 2875,2850,1651,1439,1370$, 1200, 1178, 1007, $976 \mathrm{~cm}^{-1}$; HRMS (ESI) m/z $281.0677[\mathrm{M}+\mathrm{H}]^{+}$; calculated for $\left[\mathrm{C}_{13} \mathrm{H}_{17} \mathrm{BrN}_{2}+\mathrm{H}\right]^{+}: 281.0648 ;[\alpha]_{589}{ }^{24}=-42(c=0.7, \mathrm{MeOH})$.

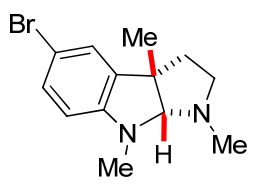

(-)-Br-deoxyeseroline (12)

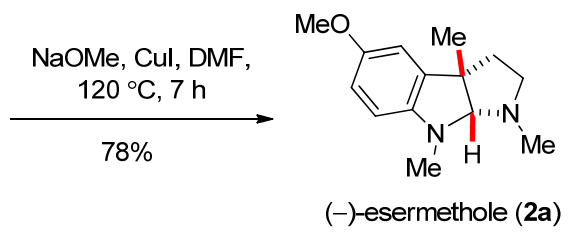


In a suspension of compound $12(18 \mathrm{mg}, 0.0642 \mathrm{mmol} ; 1.0$ equiv) and $\mathrm{CuI} \mathrm{(24}$ $\mathrm{mg}, 0.385 \mathrm{mmol} ; 2.0$ equiv) in DMF $(0.28 \mathrm{ml})$ was added sodium methoxide solution prepared from a lump of sodium (30 mg, $1.284 \mathrm{mmol} ; 20.0$ equiv) and dry $\mathrm{MeOH}(3 \mathrm{ml})$. After the resulting mixture was stirred at $120{ }^{\circ} \mathrm{C}$ for $7 \mathrm{~h}$, the reaction mixture was cooled and the insoluble materials were filtered off and filtrate was concentrated in vacuum and $\mathrm{H}_{2} \mathrm{O}$ was added to the residue. The aqueous layer extracted with $\mathrm{CH}_{2} \mathrm{Cl}_{2}(2 \times 7 \mathrm{~mL})$ and the extract was washed with brine and dried over anhydrous $\mathrm{K}_{2} \mathrm{CO}_{3}$ and concentrated under reduced pressure. The crude products were purified by flash chromatography on neutral alumina to afford the product (-)-esermethole (2a).

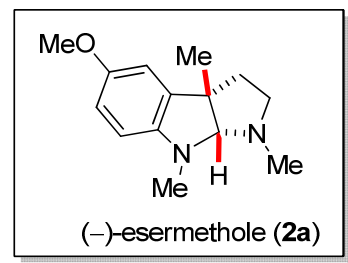

(3aS,8aR)-5-Methoxy-1,3a,8-trimethyl-1,2,3,3a,8,8a-hexahydropyrrolo[2,3-b]indole

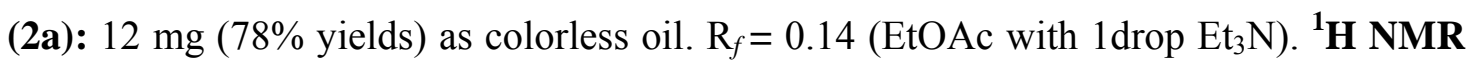

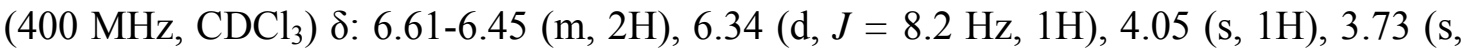
3H), 2.87 (s, 3H), 2.71 (dt, $J=5.4,10.4 \mathrm{~Hz}, 1 \mathrm{H}), 2.62$ (dd, $J=7.7,16.4 \mathrm{~Hz}, 1 \mathrm{H}), 2.52$ (s, $3 \mathrm{H}), 1.93(\mathrm{dd}, J=5.5,7.3 \mathrm{~Hz}, 2 \mathrm{H}), 1.42(\mathrm{~s}, 3 \mathrm{H}) ;{ }^{13} \mathbf{C} \mathbf{N M R}\left(100 \mathrm{MHz}, \mathrm{CDCl}_{3}\right) \delta: 153.0$, 146.6, 138.3, 112.2, 109.8, 107.5, 98.3, 56.1, 53.2, 52.8, 40.8, 38.1, 29.7, 27.4; IR (film) $v_{\max } 2964,2928,2857,1735,1498,1452,1427,1262,1219,1122,1033,1961,732 \mathrm{~cm}^{-1}$; HRMS (ESI) $\mathrm{m} / \mathrm{z} 233.1057[\mathrm{M}+\mathrm{H}]^{+}$; calculated for $\left[\mathrm{C}_{14} \mathrm{H}_{20} \mathrm{~N}_{2} \mathrm{O}+\mathrm{H}\right]^{+}: 233.1648 ;[\alpha]$ ${ }_{589}^{20.7}=-101\left(c=0.233, \mathrm{CHCl}_{3}\right)$; Lit. [Overman et al. J. Am. Chem. Soc. 1998, 120, $6488][\alpha]^{25}{ }_{\mathrm{D}}=+107(c=0.14$, benzene $)$.

First Total Synthesis of (-)-pseudophrynamine 272A (3d) and (-)pseudophrynamine 270 (3b): 


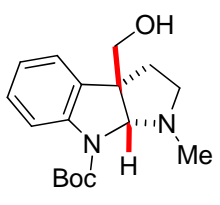

$(-)-4$

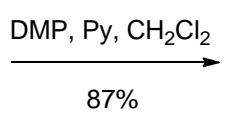

Dess-Martin periodinane ( $835 \mathrm{mg}, 1.968 \mathrm{mmol} ; 1.2$ equiv) was added to a solution of primary alcohol 4 (500 mg, $1.643 \mathrm{mmol}$; 1.0 equiv), pyridine (159 $\mu \mathrm{L}, 1.968 \mathrm{mmol} ; 1.2$ equiv) and $\mathrm{CH}_{2} \mathrm{Cl}_{2}(15 \mathrm{~mL})$ at $\mathrm{rt}$. After $1 \mathrm{~h}$, the reaction mixture was poured into a mixture of $\mathrm{CH}_{2} \mathrm{Cl}_{2}(20 \mathrm{~mL})$ and $10 \%$ aqueous $\mathrm{NaHSO}_{3}(5 \mathrm{~mL})$. The layers were separated and the aqueous layer was extracted with $\mathrm{CH}_{2} \mathrm{Cl}_{2}(2 \times 20 \mathrm{~mL})$. The combined organic layers were washed sequentially with saturated aqueous $\mathrm{NaHCO}_{3}(5 \mathrm{~mL}), \mathrm{H}_{2} \mathrm{O}$ $(20 \mathrm{~mL})$ and brine $(10 \mathrm{~mL})$, dried over $\mathrm{Na}_{2} \mathrm{SO}_{4}$ and concentrated under reduced pressure. The crude product was purified by silica gel chromatography to afford the title compounds 13.

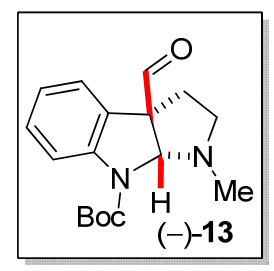

(3aR,8aR)-tert-Butyl 3a-formyl-1-methyl-1,3,3a,8a-tetrahydropyrrolo[2,3-b]indole8(2H)-carboxylate (13): $432 \mathrm{mg}$ (87\% yields) as colorless gel. $\mathrm{R}_{f}=0.3(50 \%$ EtOAc in hexane). ${ }^{1} \mathbf{H}$ NMR $\left(400 \mathrm{MHz}, \mathrm{CDCl}_{3}\right) \delta$ : $9.70(\mathrm{~s}, 1 \mathrm{H}), 7.69-7.70(\mathrm{~m}, 1 \mathrm{H}), 7.27-7.29(\mathrm{~m}$, $1 \mathrm{H}), 7.15(\mathrm{~d}, J=6.8 \mathrm{~Hz}, 1 \mathrm{H}), 7.04(\mathrm{t}, J=7.5 \mathrm{~Hz}, 1 \mathrm{H}), 5.4(\mathrm{~s}, 1 \mathrm{H}), 2.69-2.80(\mathrm{~m}, 1 \mathrm{H})$, $2.60(\mathrm{~s}, 3 \mathrm{H}), 2.54-2.57(\mathrm{~m}, 1 \mathrm{H}), 1.97-2.02(\mathrm{~m}, 1 \mathrm{H}), 1.57$ (s, 9H); ${ }^{13} \mathrm{C}$ NMR $(100 \mathrm{MHz}$, $\left.\mathrm{CDCl}_{3}\right)$ 8: 196.7, 139.7, 134.7, 129.7, 129.4, 129.3, 123.5, 116.4, 83.8, 82.0, 60.4, 53.0, 38.8, 33.1, 28.4; IR (film) $v_{\max } 2978,2916,2804,1704,1600,1485,1464,1385,1368$, 1311, 1250, 1165, 1153, 1072, 1020, $754 \mathrm{~cm}^{-1}$; HRMS (ESI) m/z $303.1717[\mathrm{M}+\mathrm{H}]^{+}$; calculated for $\left[\mathrm{C}_{17} \mathrm{H}_{22} \mathrm{~N}_{2} \mathrm{O}_{3}+\mathrm{H}\right]^{+}: 303.1703 ;[\alpha]^{20.4}{ }_{589}=-11.77\left(c=0.153, \mathrm{CHCl}_{3}\right)$. 


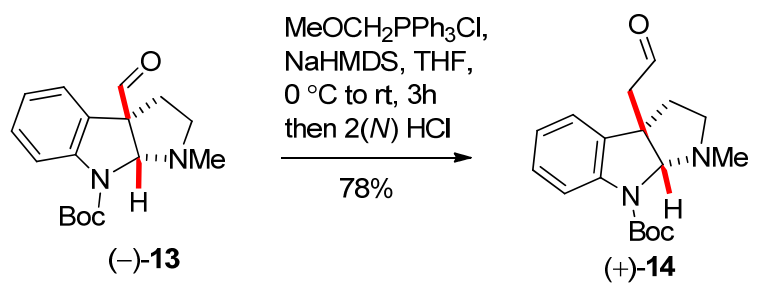

A flame-dried round-bottom flask was charged with the Wittig reagent $(3.1 \mathrm{gm}$, $9.043 \mathrm{mmol}, 1.3$ equiv) and THF $(20 \mathrm{~mL})$ and cooled to $0{ }^{\circ} \mathrm{C}$ followed by addition of NaHMDS (4.87 mL, $9.744 \mathrm{mmol}, 1.3$ equiv) dropwise. The resulting reddish brown solution was stirred at $0{ }^{\circ} \mathrm{C}$ for $30 \mathrm{~min}$ and then a solution of compound 13 (2.4 gm, 6.96 mmol; 1.0 equiv) in THF $(10 \mathrm{~mL})$ was added dropwise via syringe to the reaction vessel. The reaction mixture was stirred for another $2.5 \mathrm{~h}$ at $0{ }^{\circ} \mathrm{C}$ and quenched with $2 \mathrm{~N} \mathrm{HCl}(20$ $\mathrm{mL}$ ) at $0{ }^{\circ} \mathrm{C}$. Stirring was continued for $2 \mathrm{~h}$ and then the reaction mixture was warmed to room temperature and neutralized by the addition of saturated $\mathrm{NaHCO}_{3}(40 \mathrm{~mL})$. The resulting mixture was extracted with ethyl acetate $(3 \times 10 \mathrm{~mL})$. The combined organic layers were dried over $\mathrm{MgSO}_{4}$ and concentrated under reduced pressure. The crude product was purified by flash chromatography by silica gel to give the title compound $\mathbf{1 4}$.

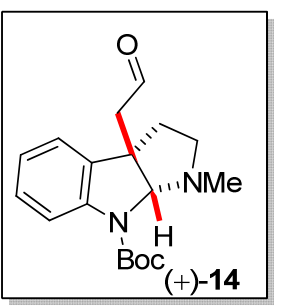

(3aR,8aR)-tert-Butyl

1-methyl-3a-(2-oxoethyl)-1,3,3a,8a-tetrahydropyrrolo[2,3b]indole-8(2H)-carboxylate (14): 1.7 gm (78\% yields) as colorless oil. $\mathrm{R}_{f}=0.33(30 \%$ EtOAc in hexane). ${ }^{1} \mathbf{H}$ NMR $\left(400 \mathrm{MHz}, \mathrm{CDCl}_{3}\right) \delta: 9.59(\mathrm{~s}, 1 \mathrm{H}), 7.63(\mathrm{~s}, 1 \mathrm{H}), 7.18(\mathrm{t}, J=$ $7.8 \mathrm{~Hz}, 1 \mathrm{H}), 7.13(\mathrm{~d}, J=7.3 \mathrm{~Hz}, 1 \mathrm{H}), 6.99$ (t, $J=7.4 \mathrm{~Hz}, 1 \mathrm{H}), 5.08(\mathrm{~s}, 1 \mathrm{H}), 2.84(\mathrm{qd}, J=$ 1.9, $16.5 \mathrm{~Hz}, 2 \mathrm{H}), 2.73-2.68(\mathrm{~m}, 1 \mathrm{H}), 2.63-2.57(\mathrm{~m}, 1 \mathrm{H}), 2.54(\mathrm{~s}, 3 \mathrm{H}), 2.15(\mathrm{t}, J=16.2$ $\mathrm{Hz}, 2 \mathrm{H}), 1.56(\mathrm{~s}, 9 \mathrm{H}) ;{ }^{13} \mathrm{C}$ NMR $\left(100 \mathrm{MHz}, \mathrm{CDCl}_{3}\right) \delta: 200.3,153.1,142.3,136.0,128.4$, 123.4, 123.3, 116.3, 87.1, 81.6, 52.7, 52.2, 37.9, 37.4, 29.7, 28.4; IR (film) $v_{\max } 2979$, 2900, 1701, 1643, 1600, 1423, 1409, 1270, 1170, $976 \mathrm{~cm}^{-1}$; HRMS (ESI) m/z 317.1782 
$[\mathrm{M}+\mathrm{H}]^{+}$; calculated for $\left[\mathrm{C}_{18} \mathrm{H}_{24} \mathrm{~N}_{2} \mathrm{O}_{3}+\mathrm{H}\right]^{+}: 317.1860 ;[\alpha]^{25.4}{ }_{589}=+4.1(c=0.137$, $\left.\mathrm{CHCl}_{3}\right)$.

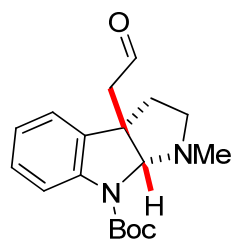

$(+)-14$

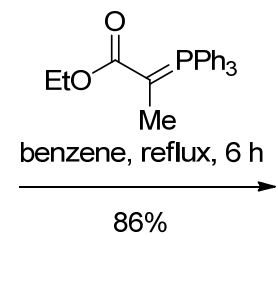

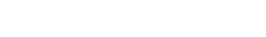

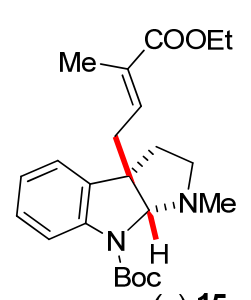

$(-)-15$

In a solution of compound 14 (1.2 gm, $3.795 \mathrm{mmol} ; 1.0$ equiv) in dry benzene (20 $\mathrm{mL}$ ), Ethyl 2-(triphenylphosphoranylidene)propionate (1.65 gm, $4.554 \mathrm{mmol} ; 1.2$ equiv) was added at room temperature and then reaction mixture stirred at $100{ }^{\circ} \mathrm{C}$ for $6 \mathrm{~h}$. Upon completion of the reactions, the reaction mixture was concentrated under reduced pressure. The crude products were purified by flash column chromatography by silica gel and afforded the product 15.

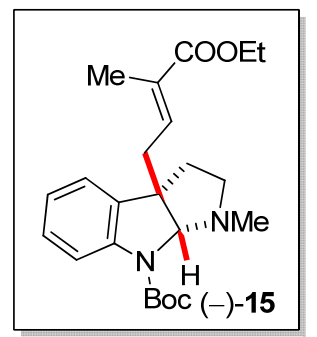

(3aS,8aR)-tert-Butyl

3a-((E)-4-ethoxy-3-methyl-4-oxobut-2-en-1-yl)-1-methyl1,3,3a,8a-tetrahydropyrrolo[2,3-b]indole-8(2H)-carboxylate $\quad(15): \quad 1.3$ gm (86\% yields) as colorless gel. $\mathrm{R}_{f}=0.6\left(30 \%\right.$ EtOAc in hexane). ${ }^{1} \mathbf{H} \mathbf{~ N M R}\left(400 \mathrm{MHz}, \mathrm{CDCl}_{3}\right) \delta$ : $7.62(\mathrm{~s}, 1 \mathrm{H}), 7.16$ (t, $J=7.7 \mathrm{~Hz}, 1 \mathrm{H}), 7.07$ (d, $J=7.3 \mathrm{~Hz}, 1 \mathrm{H}), 6.96$ (t, $J=7.4 \mathrm{~Hz}, 1 \mathrm{H})$, $6.62(\mathrm{t}, J=7.4 \mathrm{~Hz}, 1 \mathrm{H}), 4.86(\mathrm{~s}, 1 \mathrm{H}), 4.11(\mathrm{q}, J=7.0 \mathrm{~Hz}, 2 \mathrm{H}), 2.63-2.52(\mathrm{~m}, 4 \mathrm{H}), 2.50$ (s, 3H), 2.13-2.01 (m, 2H), $1.75(\mathrm{~s}, 3 \mathrm{H}), 1.53(\mathrm{~s}, 9 \mathrm{H}), 1.22(\mathrm{t}, J=7.1 \mathrm{~Hz}, 3 \mathrm{H}) ;{ }^{13} \mathbf{C} \mathbf{N M R}$ $\left(100 \mathrm{MHz}, \mathrm{CDCl}_{3}\right) \delta: 167.7,153.1,142.1,136.5,130.5,128.0,123.2,123.1,123.0$, 116.1, 87.0, 81.3, 60.5, 53.2, 38.1, 36.7, 28.4, 14.2, 12.7; IR (film) $v_{\max } 3211,2976$, 2956, 2890, 1700, 1660, 1500, 1416, 1230, 1200, 1107, $777 \mathrm{~cm}^{-1}$; HRMS (ESI) m/z 
$401.2455[\mathrm{M}+\mathrm{H}]^{+}$; calculated for $\left[\mathrm{C}_{23} \mathrm{H}_{32} \mathrm{~N}_{2} \mathrm{O}_{4}+\mathrm{H}\right]^{+}: 401.2435 ;[\alpha]^{22.5}{ }_{589}=-24.4(c=$ $\left.0.083, \mathrm{CHCl}_{3}\right)$.

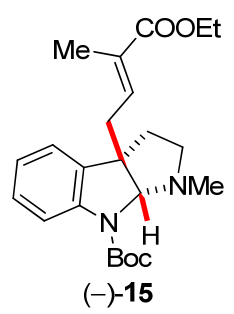

$\underset{94 \%}{\stackrel{\mathrm{LiAlH}_{4}, \text { reflux, } 12 \mathrm{~h}}{\longrightarrow}}$

$(-)-15$

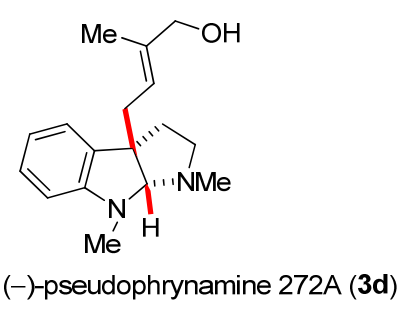

(-)-pseudophrynamine 272A (3d)

In an oven-dried round bottom flask, compound $15(100 \mathrm{mg}, 0.25 \mathrm{mmol} ; 1.0$ equiv) was taken in dry THF $(10 \mathrm{~mL})$ under argon atmosphere and the reaction vessel was cooled to $0{ }^{\circ} \mathrm{C}$. To this reaction mixture $\mathrm{LiAlH}_{4}(66.5 \mathrm{mg}, 1.748 \mathrm{mmol} ; 7.0$ equiv.) was added portion-wise. After stirring at $0{ }^{\circ} \mathrm{C}$ for 5 minutes, the reaction mixture was warmed to $23{ }^{\circ} \mathrm{C}$ and stirring continued for another 10 minutes. Then, the reaction mixture was refluxed on a preheated oil-bath maintaining the temperature to $80{ }^{\circ} \mathrm{C}$ and stirring continued for $12 \mathrm{~h}$. Upon completion of the reaction (monitoring by TLC), it was cooled to room temperature and then it was quenched with EtOAc at $0{ }^{\circ} \mathrm{C}$, basified with $4(N) \mathrm{NaOH}$ solution and extracted with EtOAc $(2 \mathrm{X} 10 \mathrm{~mL})$. The combined organic extracts were washed with saturated brine solution, dried over anhydrous $\mathrm{K}_{2} \mathrm{CO}_{3}$, and concentrated under reduced pressure. The crude products were purified by flash chromatography by basic alumina and afforded the product (-)-pseudophrynamine $272 \mathrm{~A}$ 3d.

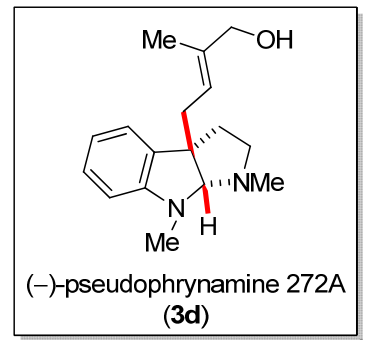

(E)-4-((3aS,8aR)-1,8-Dimethyl-1,2,3,3a,8,8a-hexahydropyrrolo[2,3-b]indol-3a-yl)-2methylbut-2-en-1-ol (3d): $64 \mathrm{mg}$ (94\% yields) as colorless gel. $\mathrm{R}_{f}=0.12$ (EtOAc in one drop $\left.\mathrm{Et}_{3} \mathrm{~N}\right) .{ }^{1} \mathbf{H} \mathbf{N M R}\left(400 \mathrm{MHz}, \mathrm{CDCl}_{3}\right) \delta: 7.08-7.04(\mathrm{~m}, 1 \mathrm{H}), 6.96(\mathrm{~d}, J=6.9 \mathrm{~Hz}, 1 \mathrm{H})$, 
$6.65(\mathrm{t}, J=7.3 \mathrm{~Hz}, 1 \mathrm{H}), 6.39(\mathrm{~d}, J=7.8 \mathrm{~Hz}, 1 \mathrm{H}), 5.32(\mathrm{t}, J=6.8 \mathrm{~Hz}, 1 \mathrm{H}), 4.14(\mathrm{~s}, 1 \mathrm{H})$, $3.90(\mathrm{~s}, 2 \mathrm{H}), 2.90(\mathrm{~s}, 3 \mathrm{H}), 2.69-2.62(\mathrm{~m}, 1 \mathrm{H}), 2.50-2.45(\mathrm{~m}, 2 \mathrm{H}), 2.42(\mathrm{~s}, 3 \mathrm{H}), 2.09-1.99$ (m, 2H), 1.88-1.83 (m, 1H), $1.61(\mathrm{~s}, 3 \mathrm{H}) ;{ }^{13} \mathbf{C}$ NMR (100 MHz, $\left.\mathrm{CDCl}_{3}\right) \delta: 152.7,137.7$, 135.4, 127.9, 122.7, 120.7, 117.7, 106.8, 93.5, 68.3, 57.1, 52.7, 38.2, 37.5, 37.4, 37.0, 14.2; IR (film) $v_{\max } 3461$ (br), 2950, 2879, 1659, 1605, 1495, 1308, 1129, $760 \mathrm{~cm}^{-1}$; HRMS (ESI) m/z $273.1989[\mathrm{M}+\mathrm{H}]^{+}$; calculated for $\left[\mathrm{C}_{17} \mathrm{H}_{24} \mathrm{~N}_{2} \mathrm{O}+\mathrm{H}\right]^{+}: 273.1961 ;[\alpha]^{25.1}$ ${ }_{589}=-22.9\left(c=0.127, \mathrm{CHCl}_{3}\right)$.

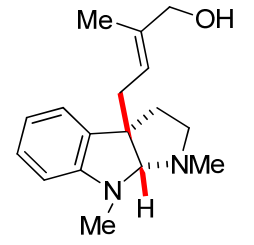

(-)-pseudophrynamine 272A (3d)

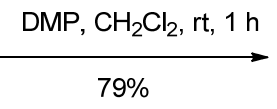

(-)-pseudophrynamine 270 (3b)

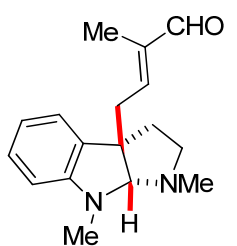

Dess-Martin periodinane (153 $\mathrm{mg}, 0.370 \mathrm{mmol} ; 2.0$ equiv) was added to a solution of allylic alcohol $3 \mathbf{d}\left(50 \mathrm{mg}, 0.185 \mathrm{mmol} ; 1.0\right.$ equiv) in $\mathrm{CH}_{2} \mathrm{Cl}_{2}(15 \mathrm{~mL})$ at $\mathrm{rt}$. After $1 \mathrm{~h}$, the reaction mixture was poured into a mixture of $\mathrm{CH}_{2} \mathrm{Cl}_{2}(10 \mathrm{~mL})$ and $10 \%$ aqueous $\mathrm{NaHSO}_{3}(0.5 \mathrm{~mL})$. The layers were separated and the aqueous layer was extracted with $\mathrm{CH}_{2} \mathrm{Cl}_{2}(3 \mathrm{X} 10 \mathrm{~mL})$. The combined organic layers were washed sequentially with saturated aqueous $\mathrm{NaHCO}_{3}(0.5 \mathrm{~mL}), \mathrm{H}_{2} \mathrm{O}(2 \mathrm{~mL})$ and brine $(1 \mathrm{~mL})$, dried over $\mathrm{Na}_{2} \mathrm{SO}_{4}$ and concentrated under reduced pressure. The crude product was purified by neutral alumina to afford the title compounds (-)-pseudophrynamine 270 (3b).

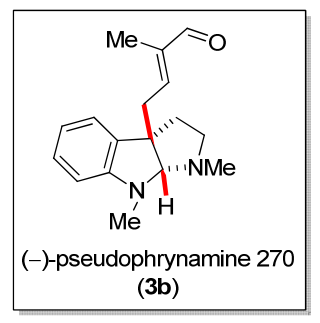

(E)-4-((3aS,8aR)-1,8-dimethyl-1,2,3,3a,8,8a-hexahydropyrrolo[2,3-b]indol-3a-yl)-2methylbut-2-enal (3b): $39 \mathrm{mg}$ (79\% yields) as yellow gel. $\mathrm{R}_{f}=0.28$ (EtOAc in one drop 
$\mathrm{Et}_{3} \mathrm{~N} .{ }^{1} \mathbf{H}$ NMR $\left(400 \mathrm{MHz}, \mathrm{CDCl}_{3}\right) \delta: 9.28(\mathrm{~s}, 1 \mathrm{H}), 7.12-7.08(\mathrm{~m}, 1 \mathrm{H}), 6.98(\mathrm{~d}, J=7.0$ $\mathrm{Hz}, 1 \mathrm{H}), 6.68$ (t, $J=7.3 \mathrm{~Hz}, 1 \mathrm{H}), 6.43$ (d, $J=7.9 \mathrm{~Hz}, 1 \mathrm{H}), 6.31-6.26$ (m, 1H), 4.17 (s, $1 \mathrm{H}), 2.91(\mathrm{~s}, 3 \mathrm{H}), 2.80$ (d, J = 7.2 Hz, 2H), 2.78-2.73 (m, 1H), 2.61-2.56 (m, 1H), 2.50 (s, 3H), 2.15-2.08 (m, 1H), 2.05-1.99 (m, 1H), $1.72(\mathrm{~s}, 3 \mathrm{H}) ;{ }^{13} \mathbf{C ~ N M R}\left(100 \mathrm{MHz}, \mathrm{CDCl}_{3}\right) \delta$ : 195.1, 152.3, 150.3, 141.0, 133.6, 128.4, 122.6, 118.1, 107.1, 94.2, 56.4, 52.5, 39.3, 39.1, 36.6, 29.7, 9.7; IR (film) $v_{\max }$ 3044, 2932, 2860, 1676, 1477, 1024, 918, $735 \mathrm{~cm}^{-1}$; HRMS (ESI) $\mathrm{m} / \mathrm{z} 271.1818[\mathrm{M}+\mathrm{H}]^{+}$; calculated for $\left[\mathrm{C}_{17} \mathrm{H}_{22} \mathrm{~N}_{2} \mathrm{O}+\mathrm{H}\right]^{+}: 271.1805$; $[\alpha]$ ${ }^{23.9}{ }_{589}=-41.4\left(c=0.217, \mathrm{CHCl}_{3}\right)$. 


\section{$\underline{{ }^{1} \mathrm{H},{ }^{13} \mathrm{C}-\mathrm{NMR} \text {, Mass and HPLC traces }}$}
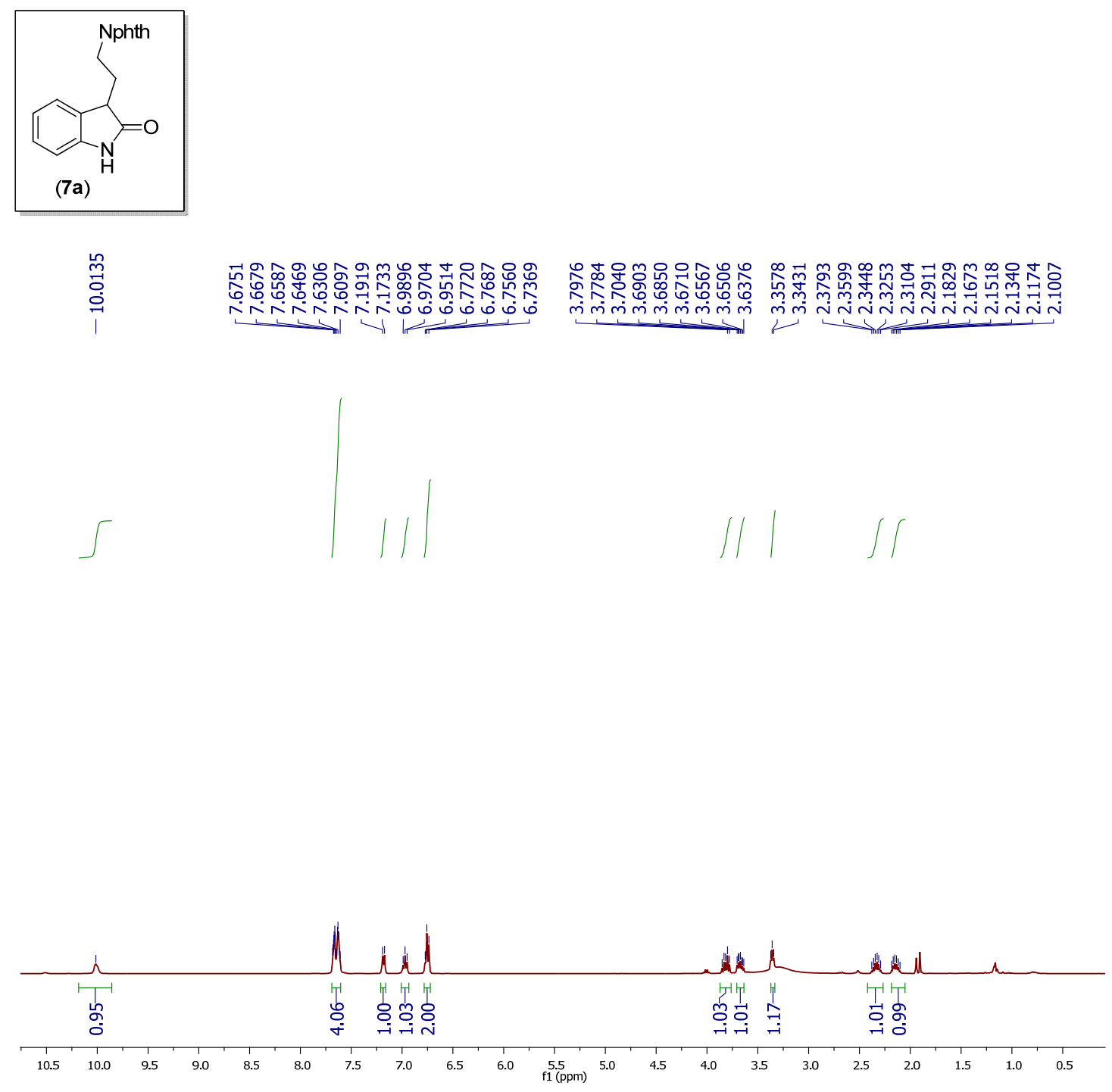

${ }^{1} \mathrm{H}$ NMR [400 MHz, DMSO-d $\left.\mathrm{d}_{6}: \mathrm{CDCl}_{3}(1: 6)\right]$ of compound (7a) 


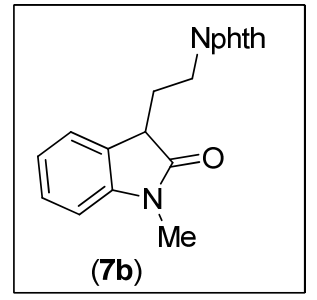

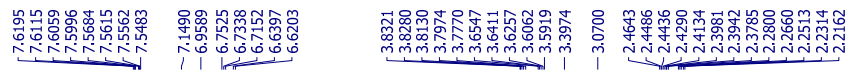

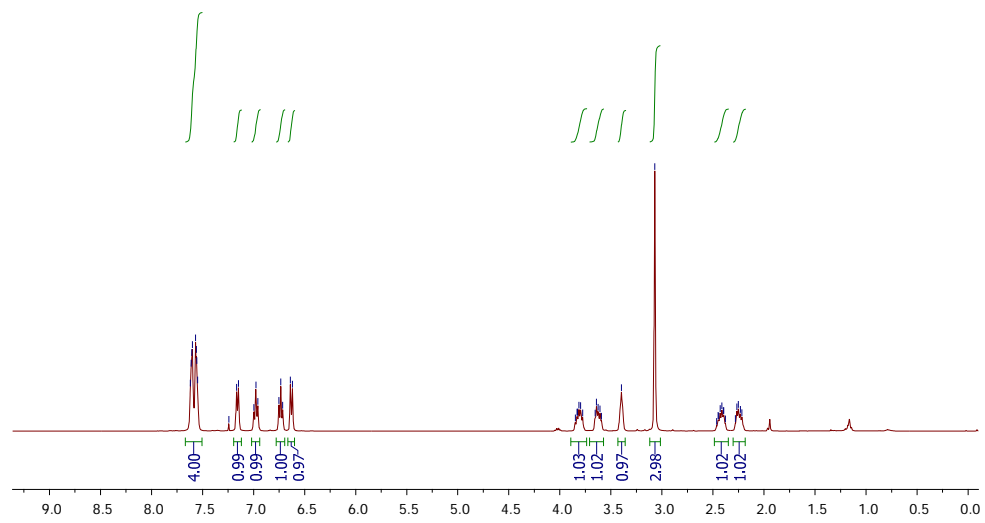

${ }^{1} \mathrm{H}$ NMR (400 MHz, $\mathrm{CDCl}_{3}$ ) of compound (7b)

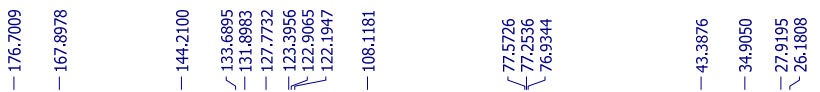

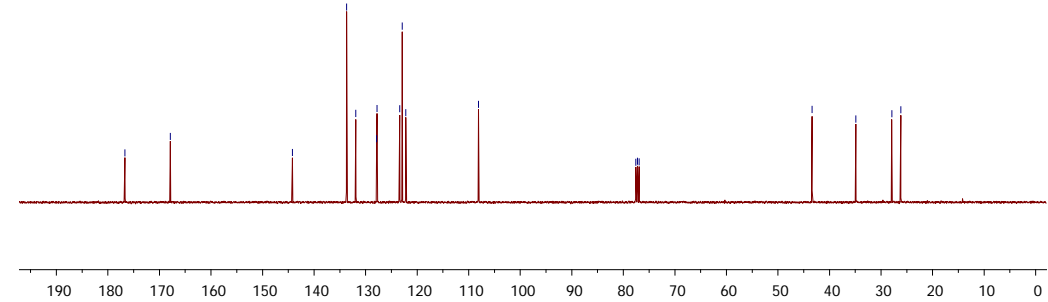

${ }^{13} \mathrm{C}$ NMR $\left(100 \mathrm{MHz}, \mathrm{CDCl}_{3}\right)$ of compound (7b) 


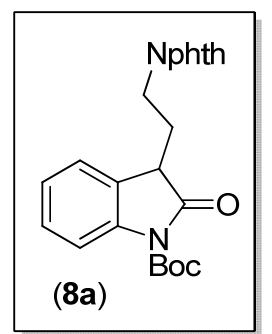

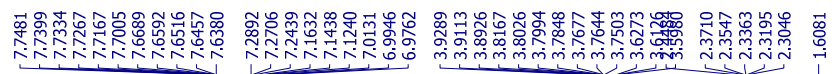

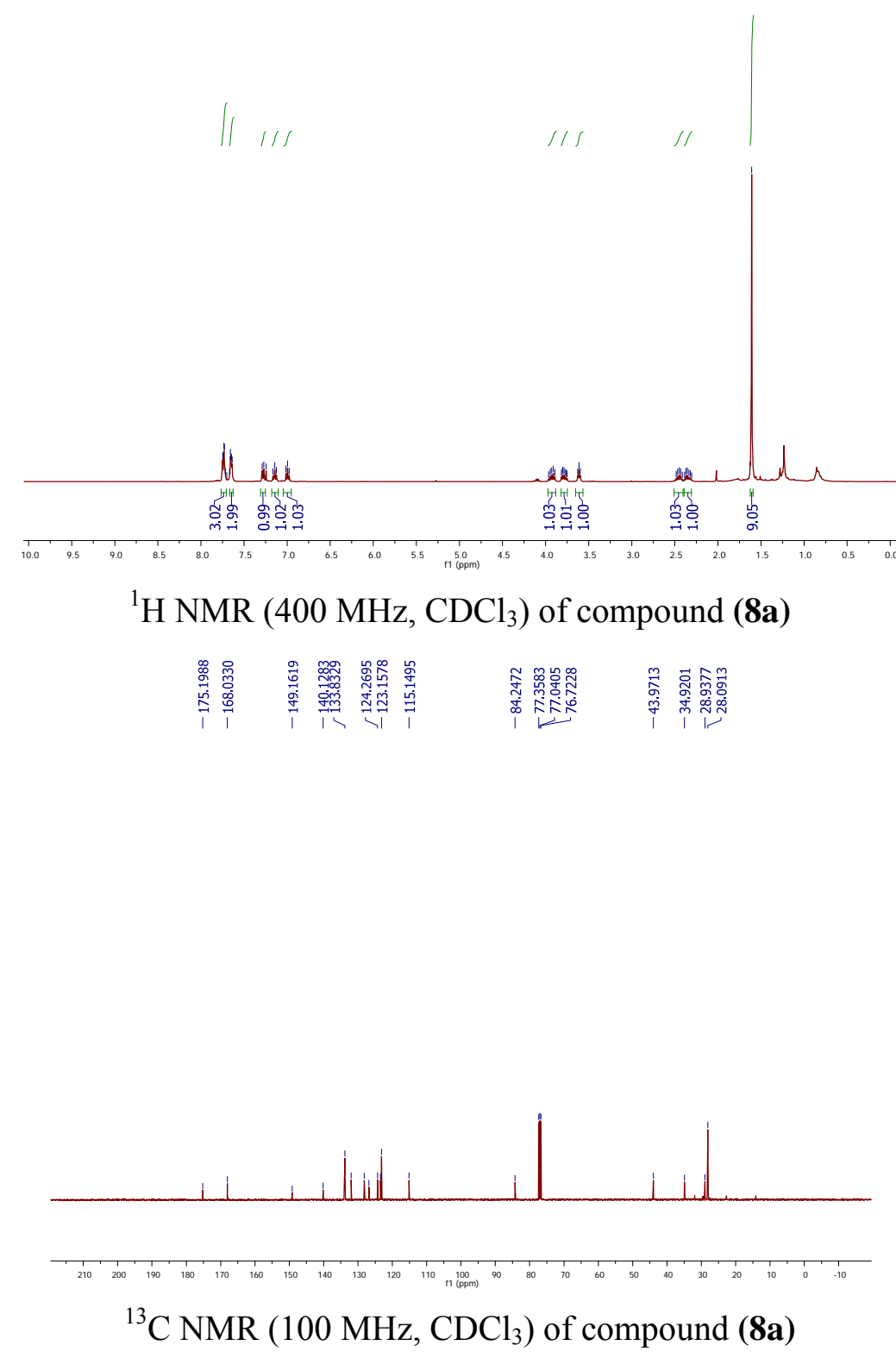




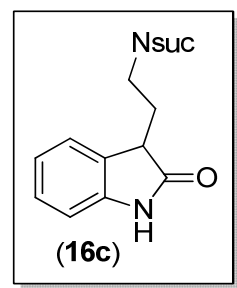

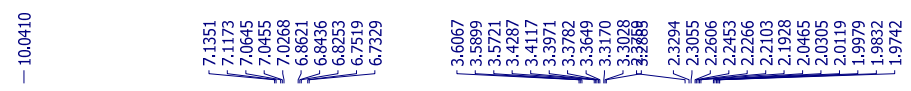

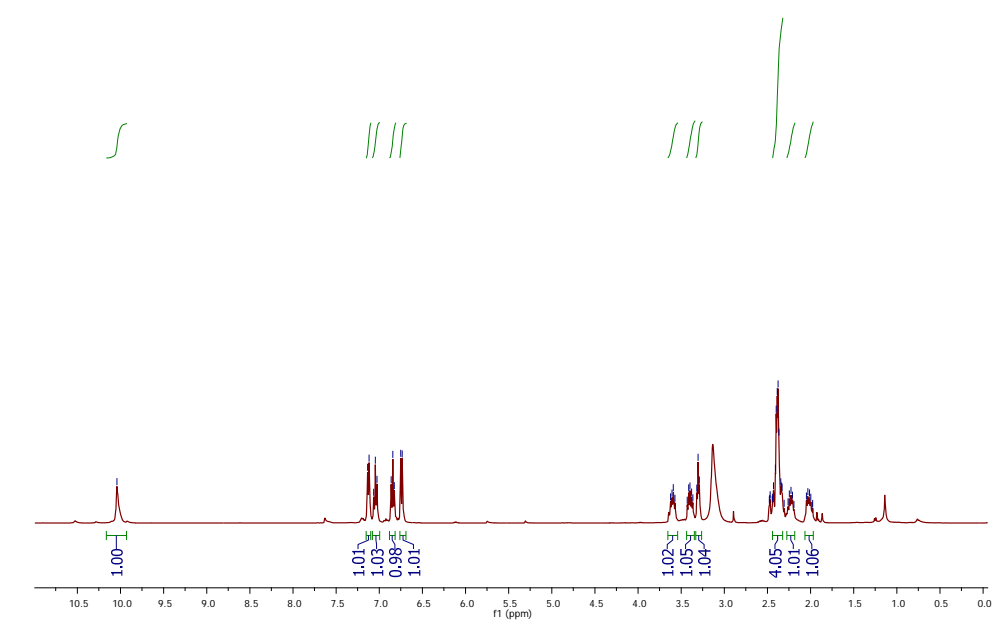

${ }^{1} \mathrm{H}$ NMR $\left[400 \mathrm{MHz}\right.$, DMSO-d $\left.\mathrm{d}_{6}: \mathrm{CDCl}_{3}(1: 6)\right]$ of compound (16c)

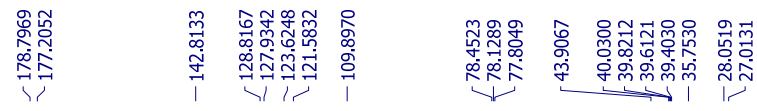

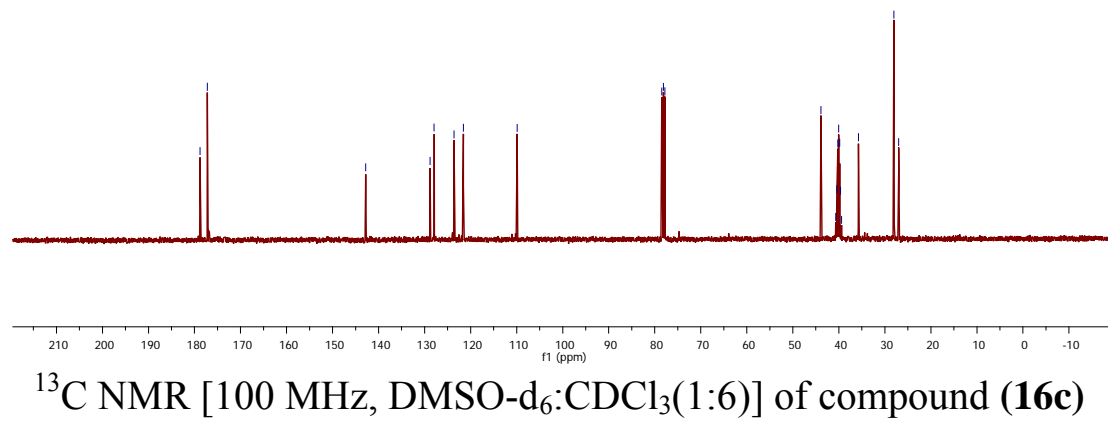




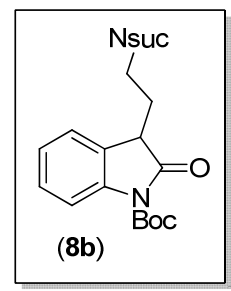

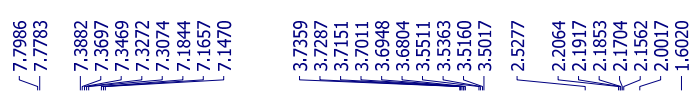

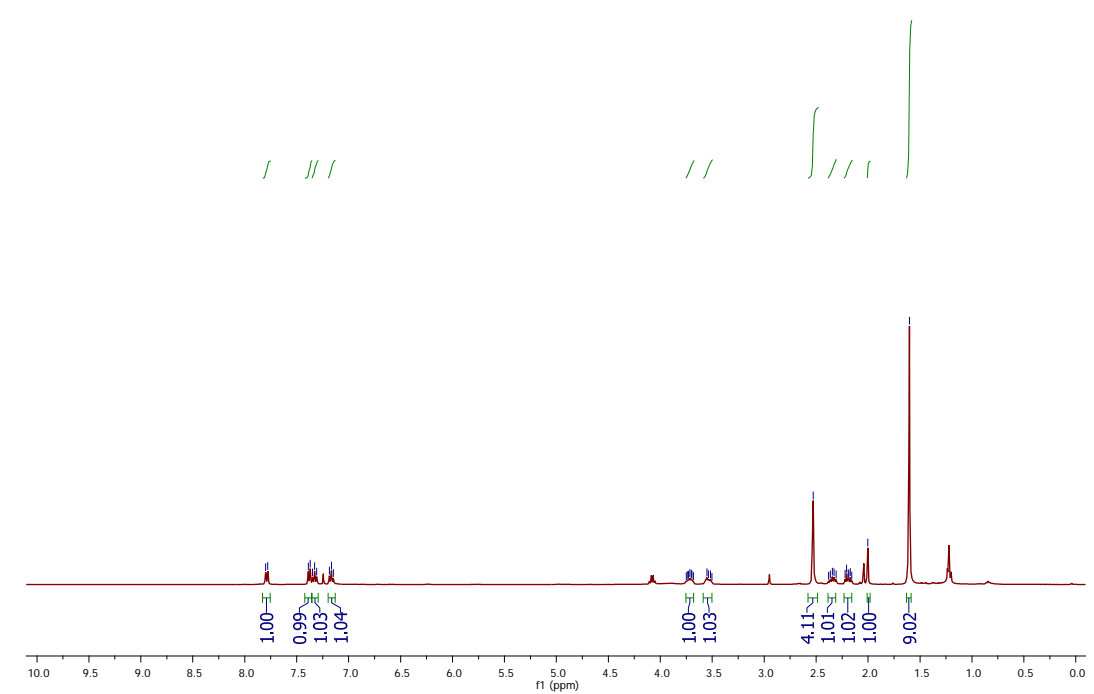

${ }^{1} \mathrm{H}$ NMR (400 MHz, $\mathrm{CDCl}_{3}$ ) of compound (8b)

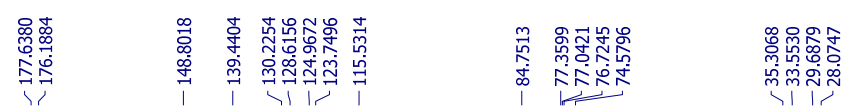
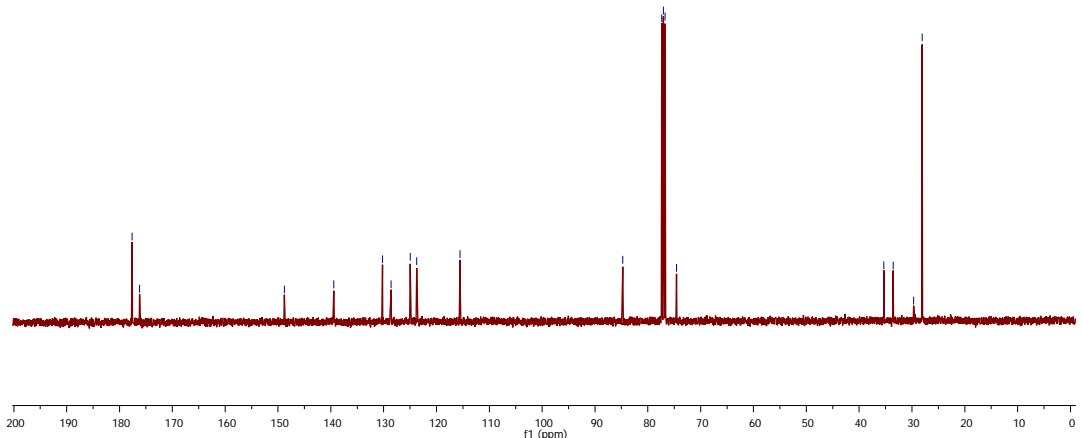

${ }^{13} \mathrm{C}$ NMR $\left(100 \mathrm{MHz}, \mathrm{CDCl}_{3}\right)$ of compound (8b) 


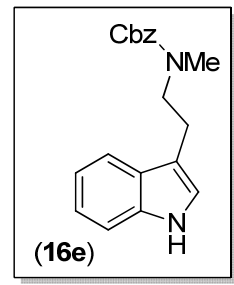

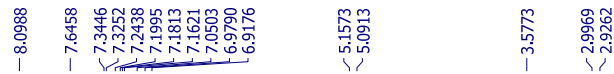
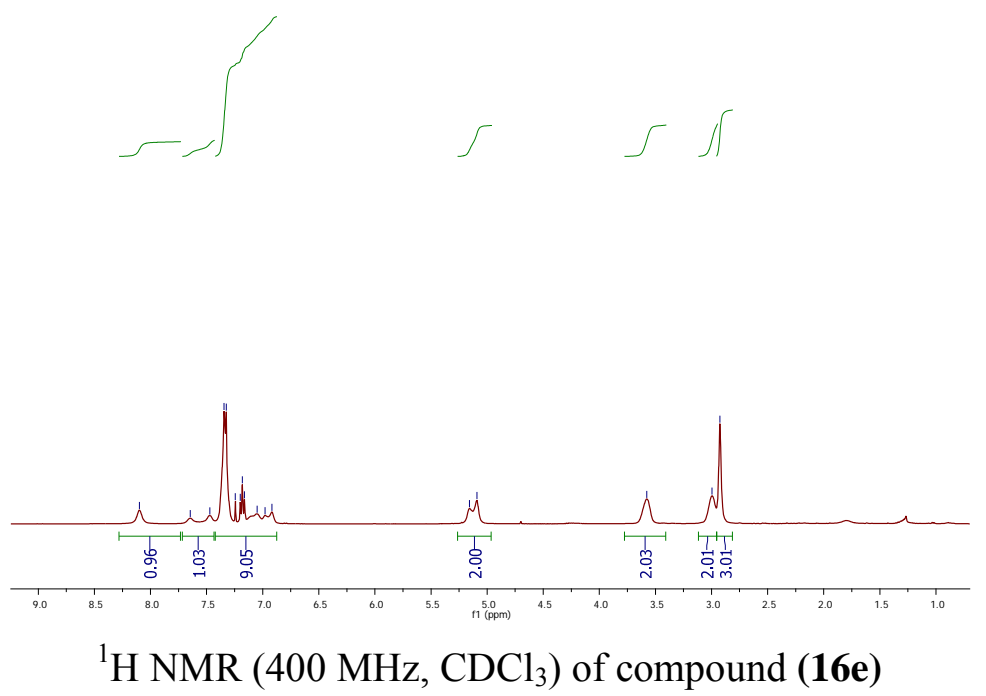

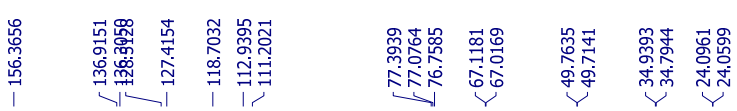
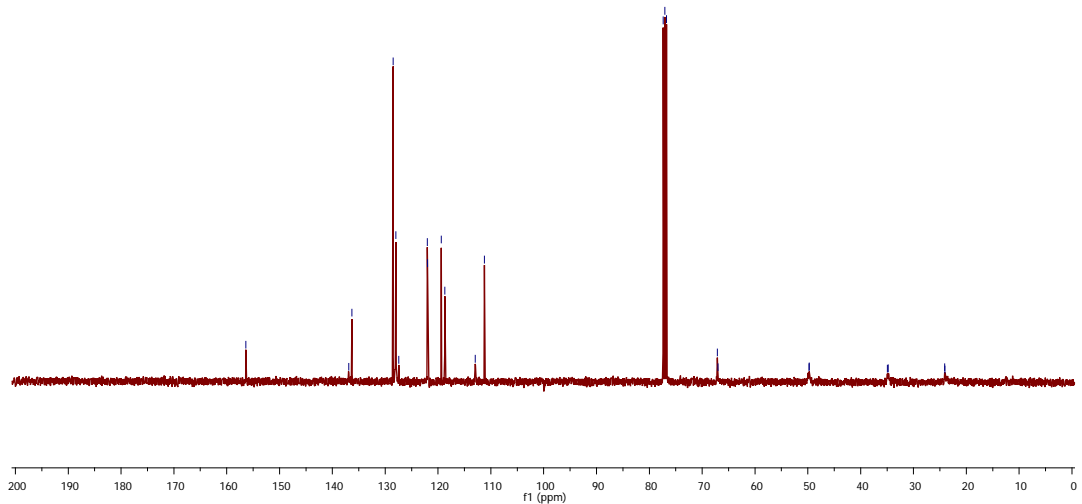

${ }^{13} \mathrm{C} \mathrm{NMR}\left(100 \mathrm{MHz}, \mathrm{CDCl}_{3}\right)$ of compound (16e) 


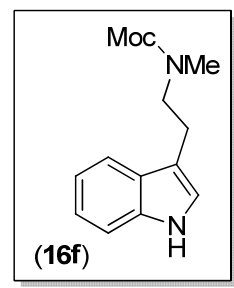

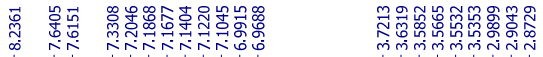
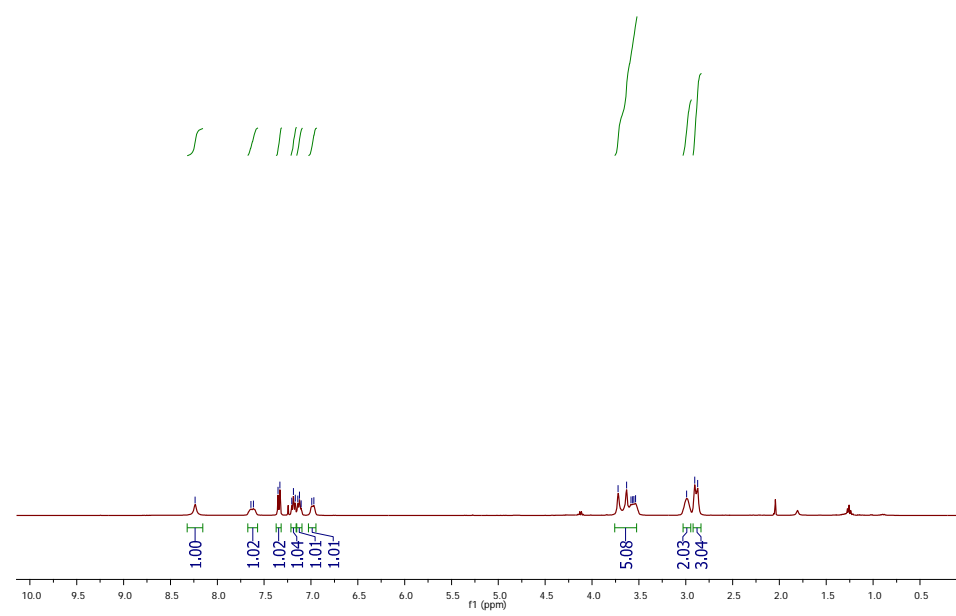

${ }^{1} \mathrm{H}$ NMR (400 MHz, $\mathrm{CDCl}_{3}$ ) of compound (16f)
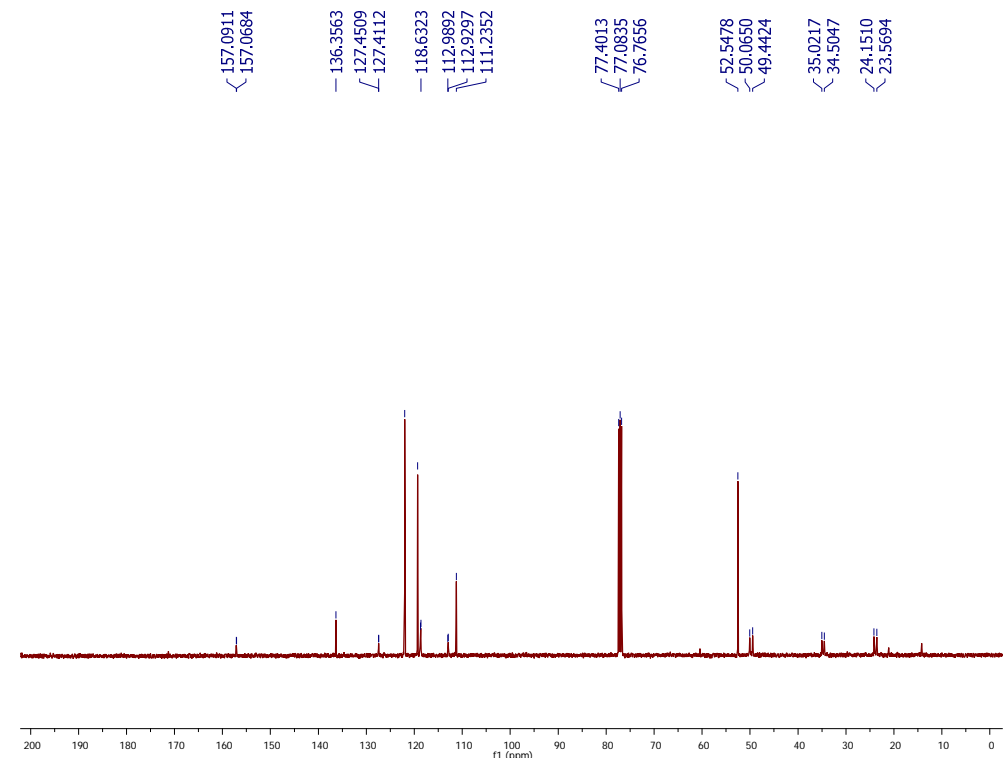

${ }^{13} \mathrm{C} \mathrm{NMR}\left(100 \mathrm{MHz}, \mathrm{CDCl}_{3}\right)$ of compound (16f) 


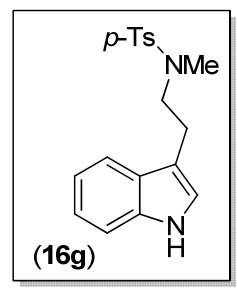

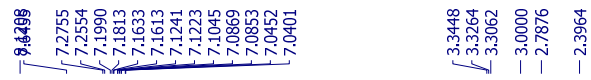

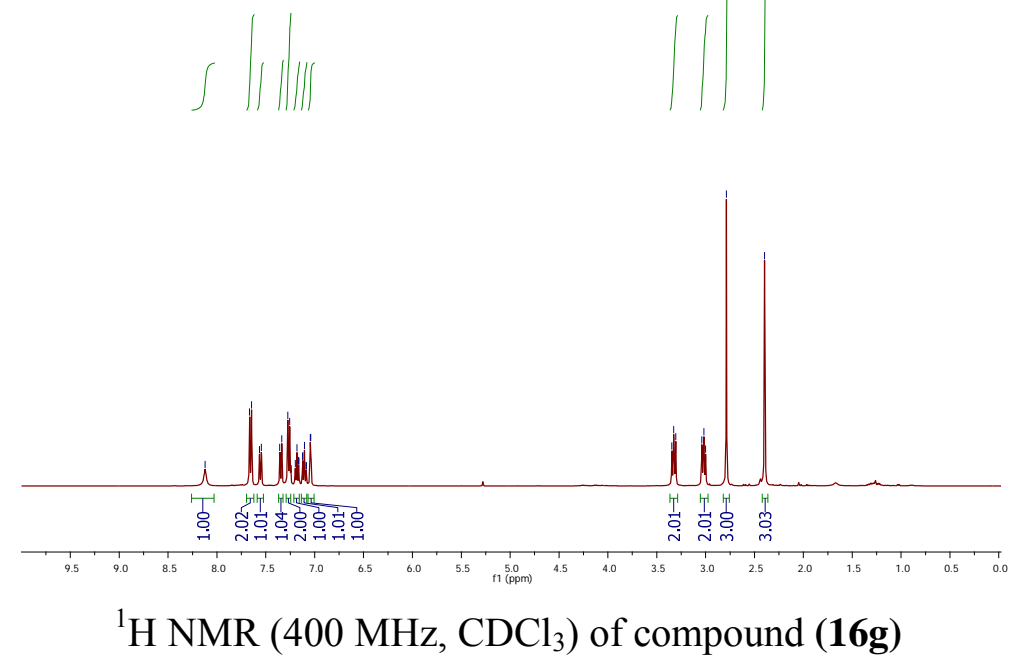

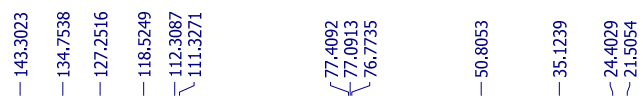
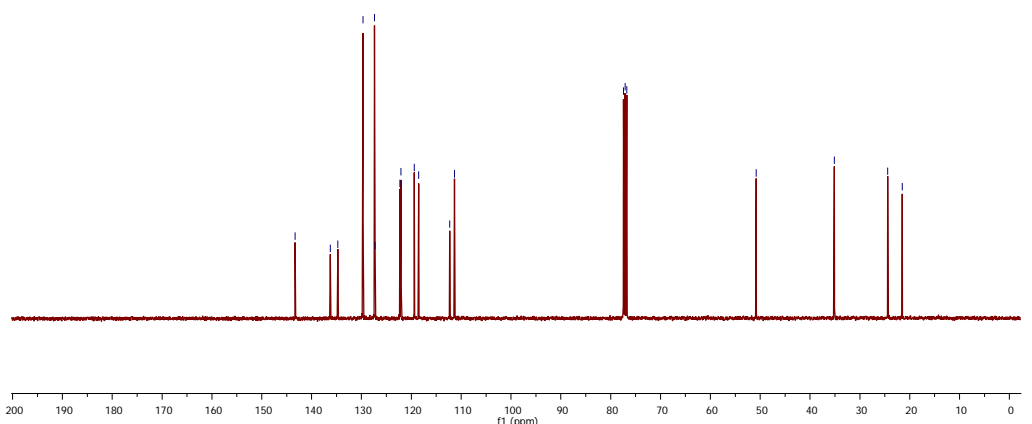

${ }^{13} \mathrm{C}$ NMR $\left(100 \mathrm{MHz}, \mathrm{CDCl}_{3}\right)$ of compound (16g) 


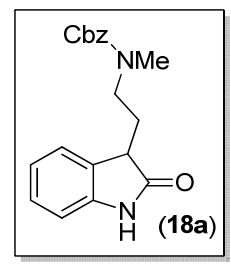

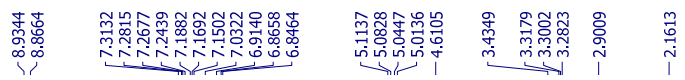

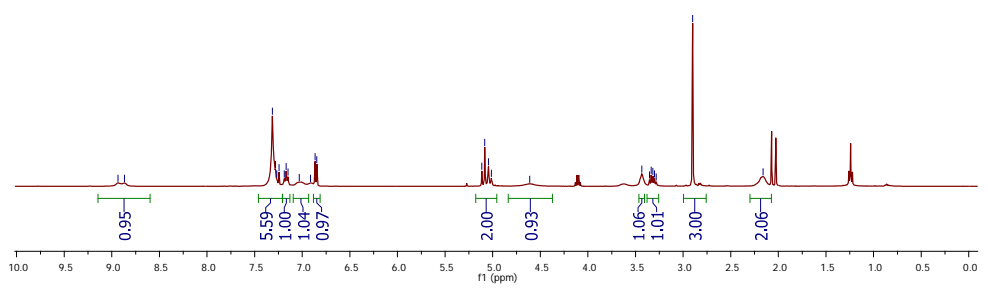

${ }^{1} \mathrm{H}$ NMR (400 MHz, $\mathrm{CDCl}_{3}$ ) of compound (18a)
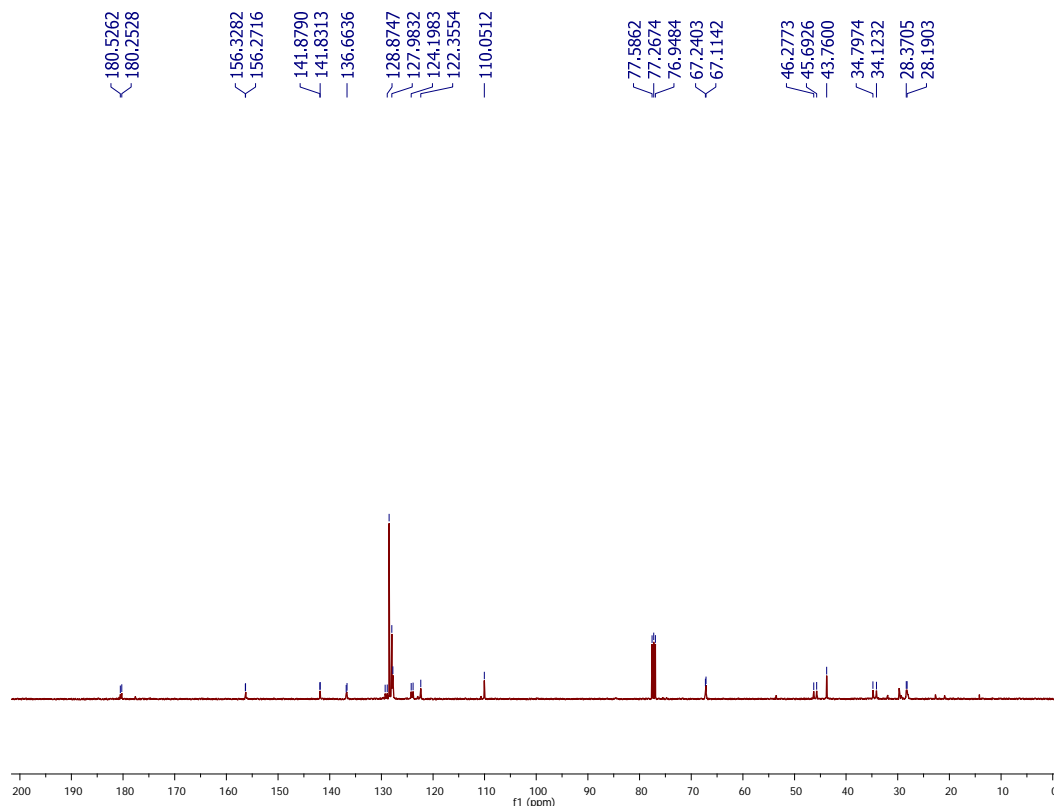

${ }^{13} \mathrm{C}$ NMR (100 MHz, $\mathrm{CDCl}_{3}$ ) of compound (18a) 

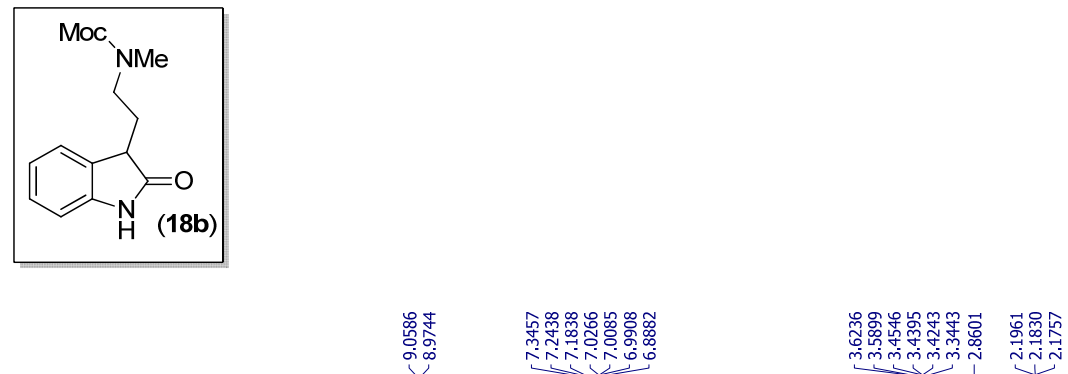

,i
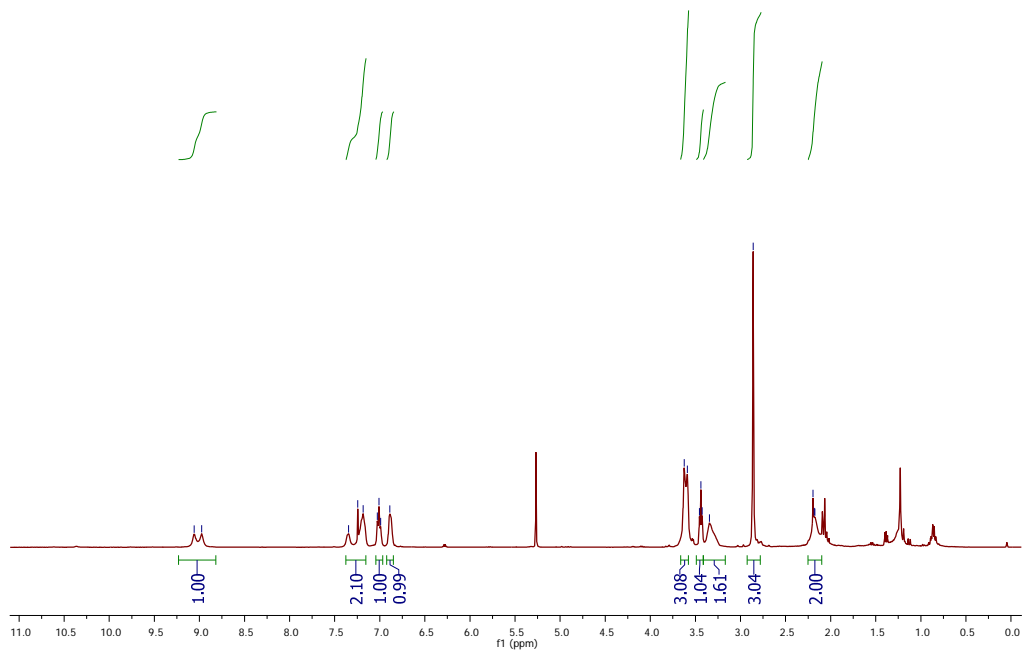

${ }^{1} \mathrm{H}$ NMR (400 MHz, $\mathrm{CDCl}_{3}$ ) of compound (18b)

\begin{tabular}{|c|c|c|c|c|c|c|}
\hline 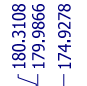 & ठั. & 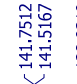 & 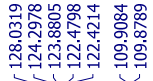 & 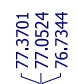 & 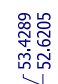 & 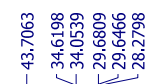 \\
\hline
\end{tabular}
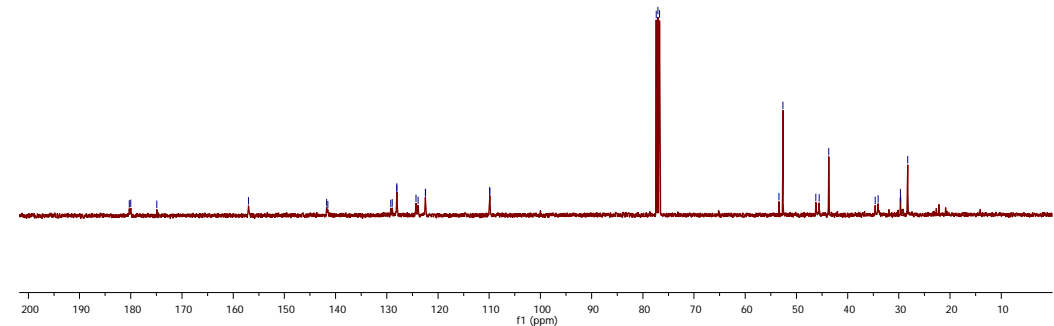

${ }^{13} \mathrm{C}$ NMR $\left(100 \mathrm{MHz}, \mathrm{CDCl}_{3}\right)$ of compound (18b) 


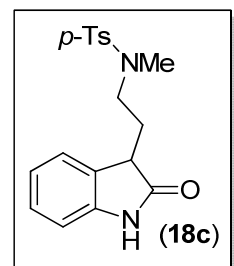

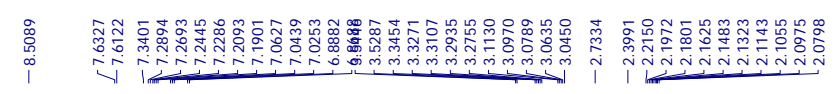
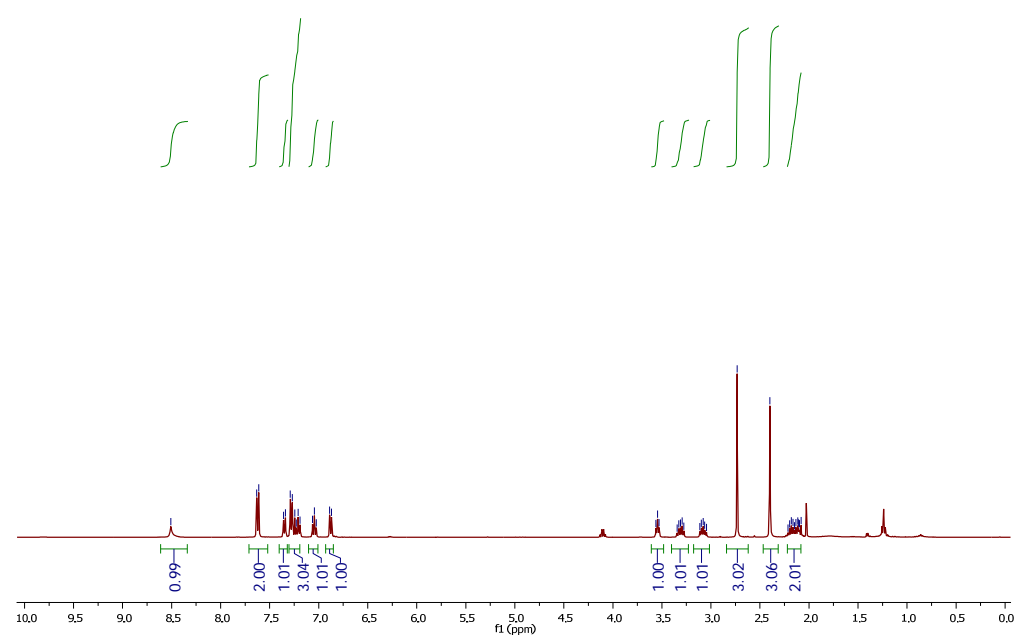

${ }^{1} \mathrm{H}$ NMR (400 MHz, $\mathrm{CDCl}_{3}$ ) of compound (18c)

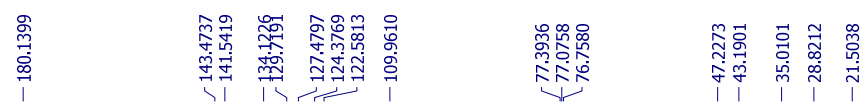
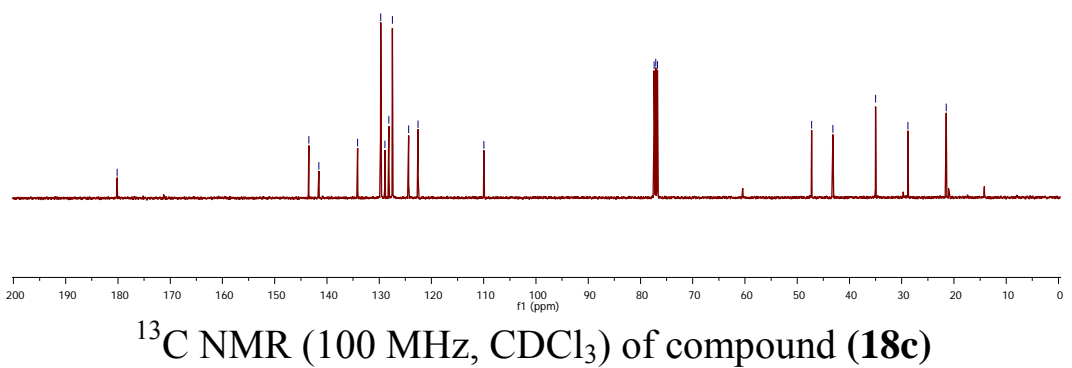


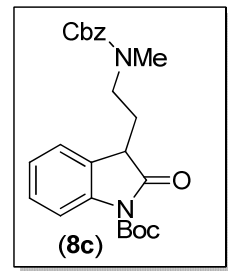

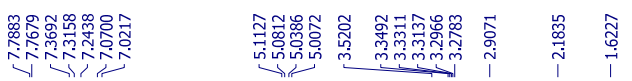

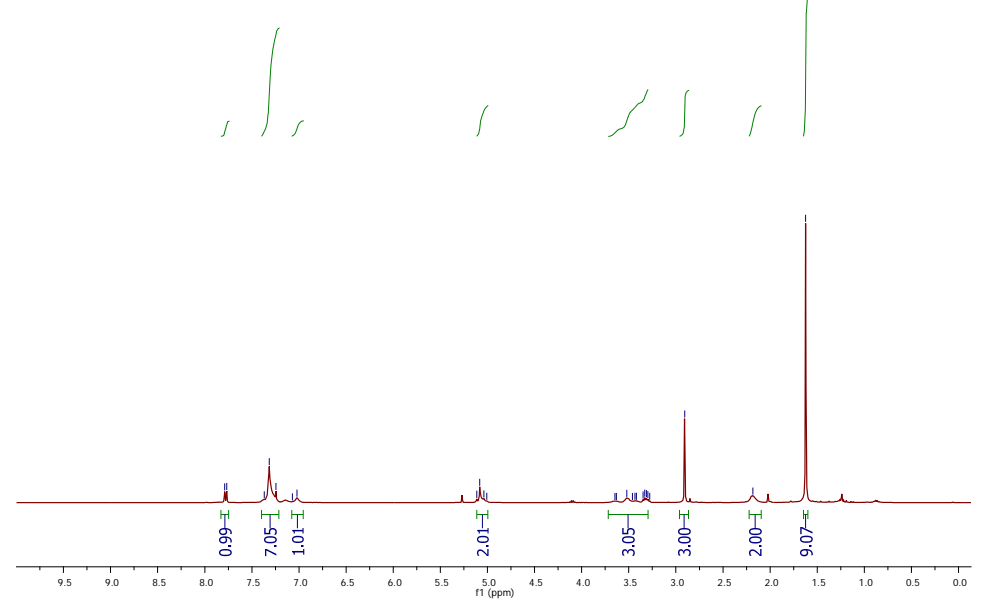

${ }^{1} \mathrm{H}$ NMR (400 MHz, $\mathrm{CDCl}_{3}$ ) of compound (8c)

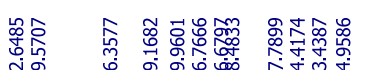

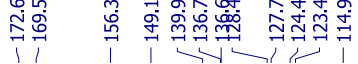
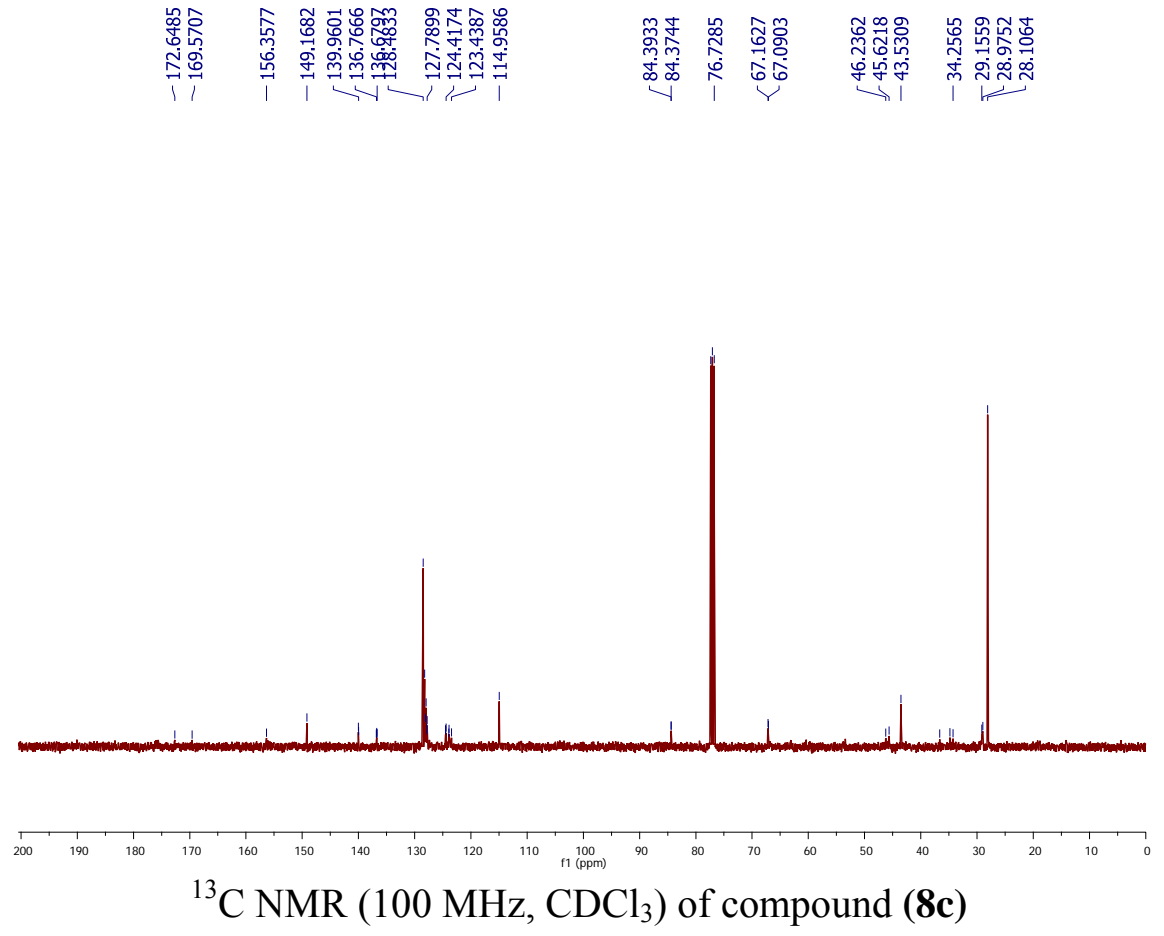

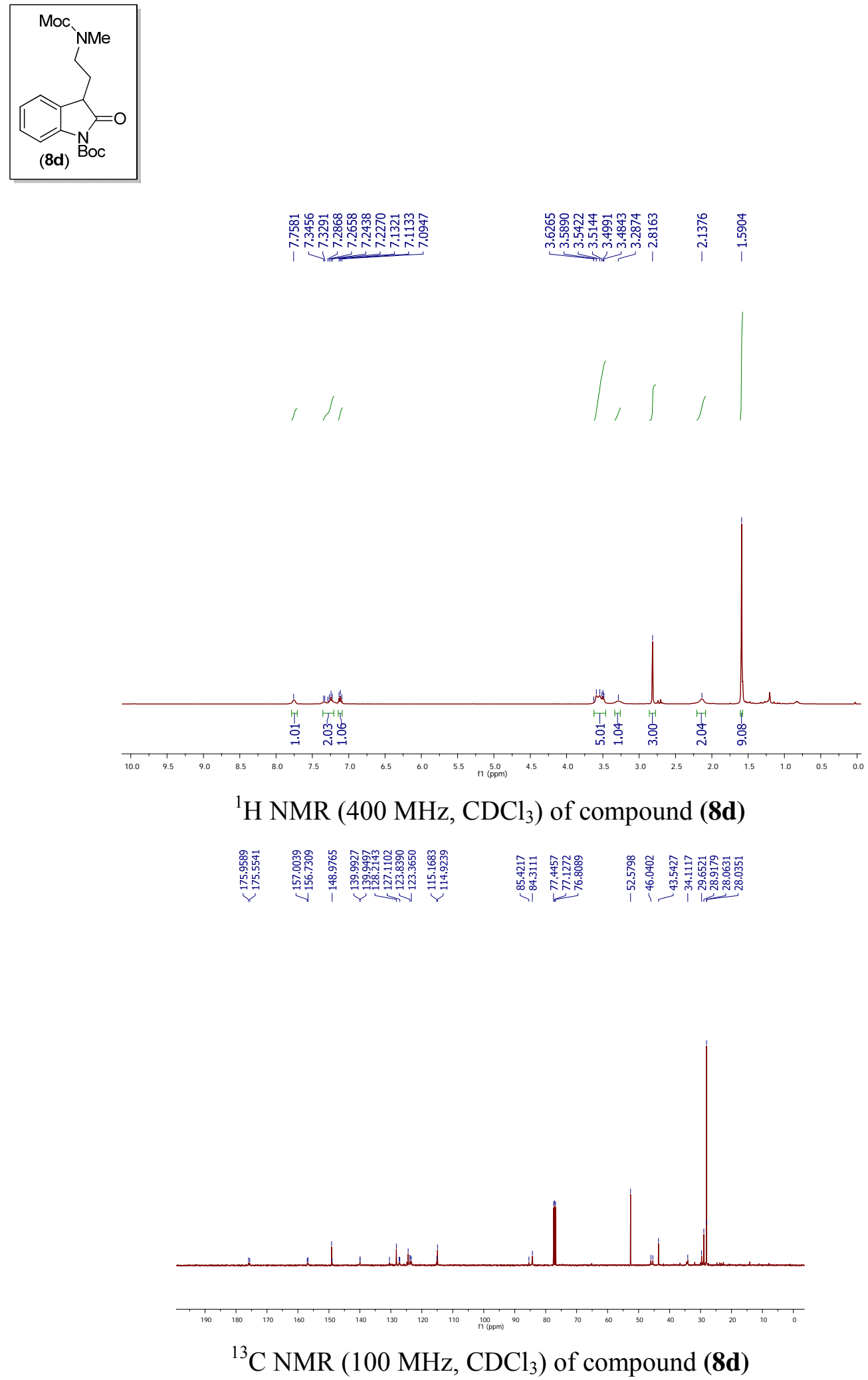


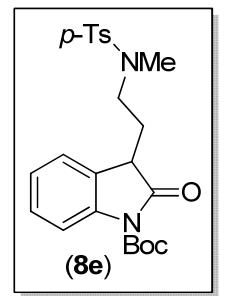

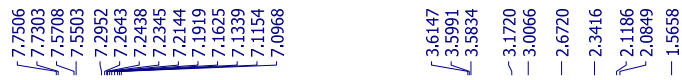

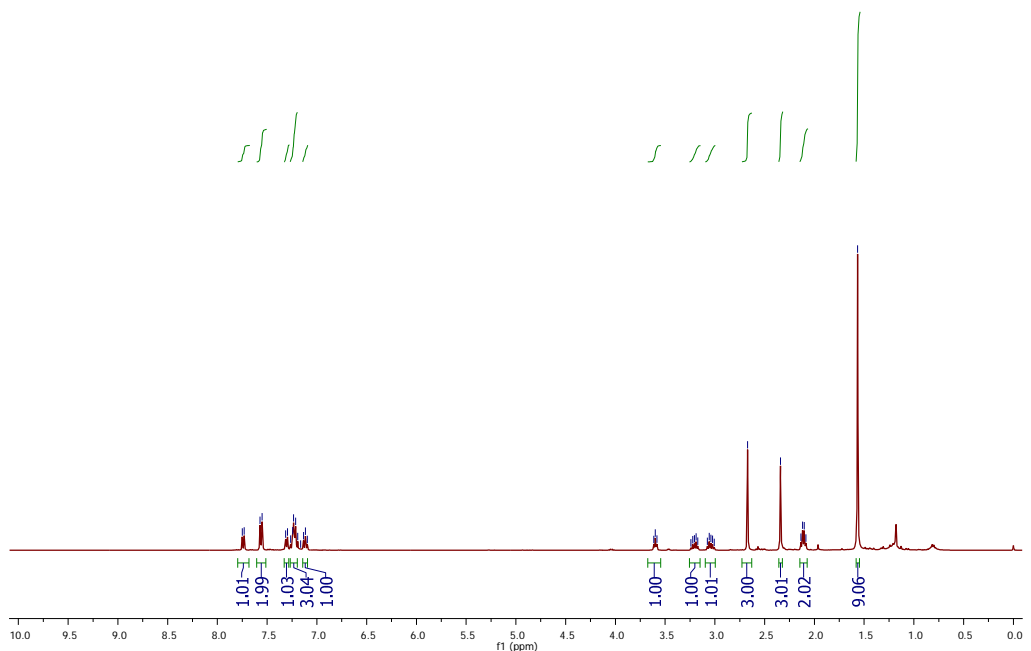

${ }^{1} \mathrm{H}$ NMR (400 MHz, $\mathrm{CDCl}_{3}$ ) of compound (8e)

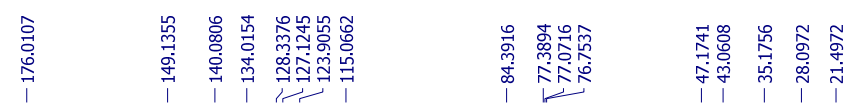

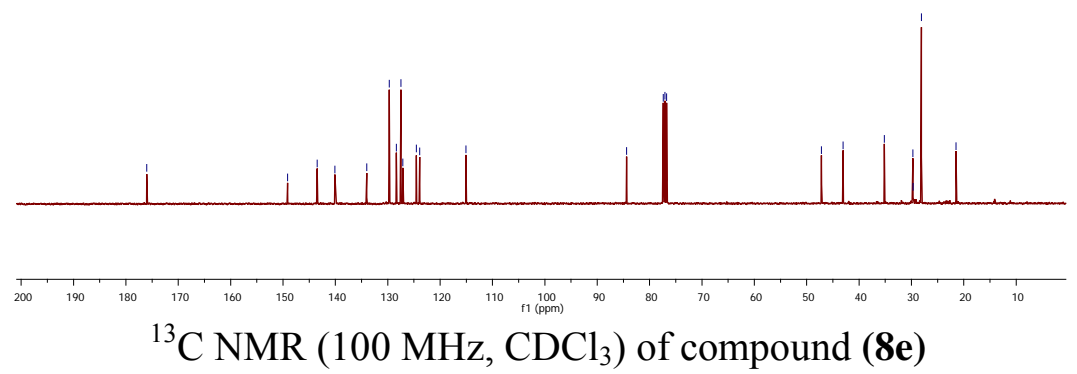




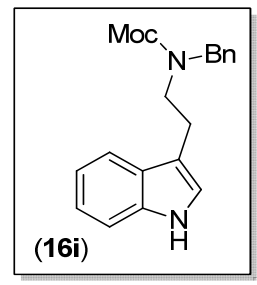

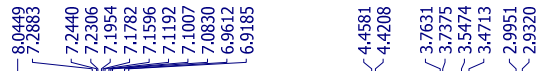
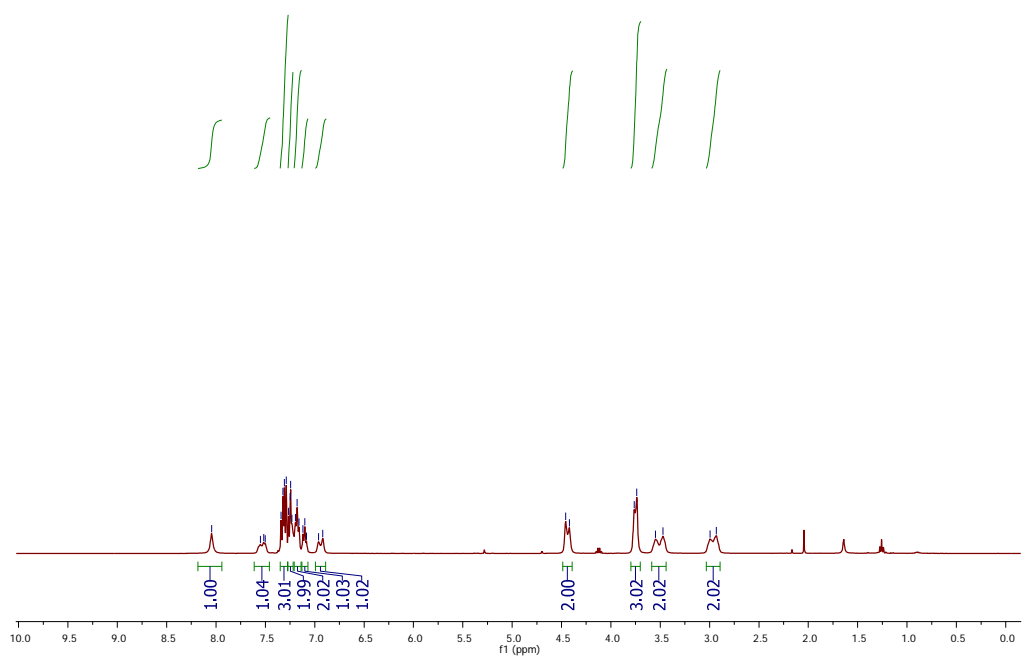

${ }^{1} \mathrm{H}$ NMR (400 MHz, $\mathrm{CDCl}_{3}$ ) of compound (16i)
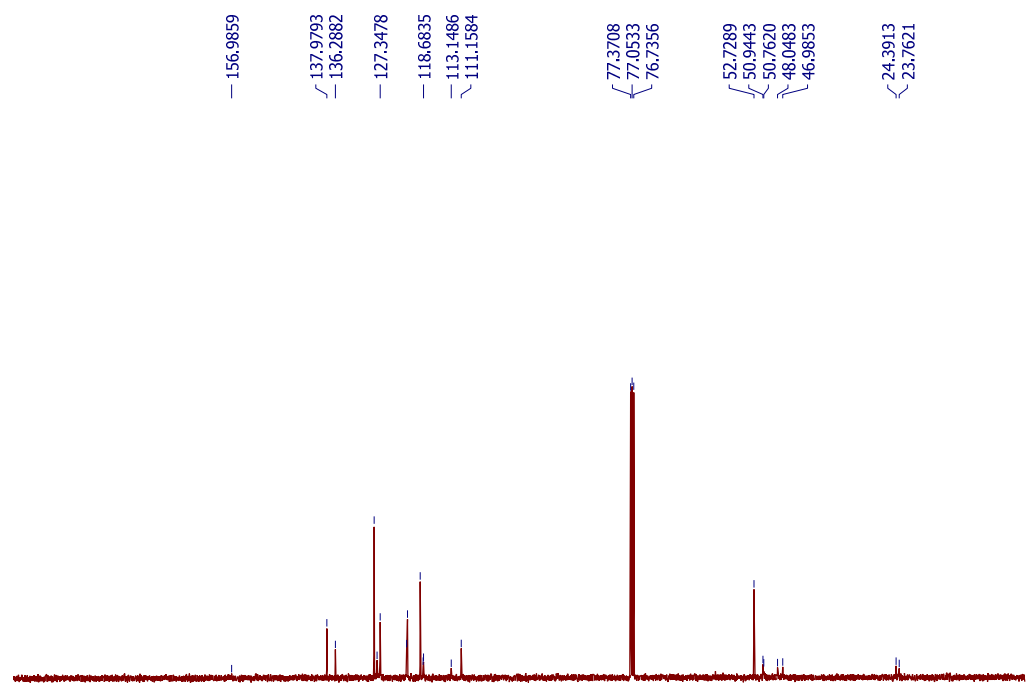

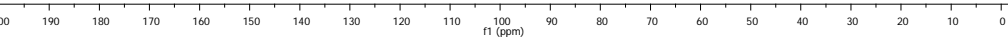

${ }^{13} \mathrm{C}$ NMR $\left(100 \mathrm{MHz}, \mathrm{CDCl}_{3}\right)$ of compound (16i) 


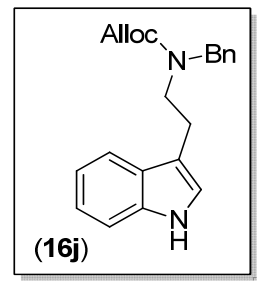

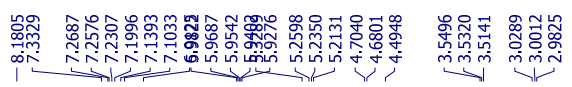

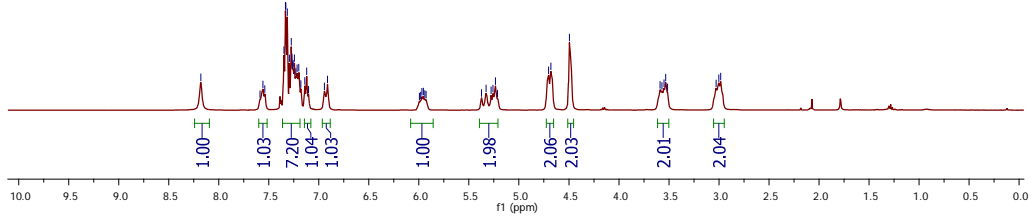

${ }^{1} \mathrm{H}$ NMR (400 MHz, $\mathrm{CDCl}_{3}$ ) of compound (16j)

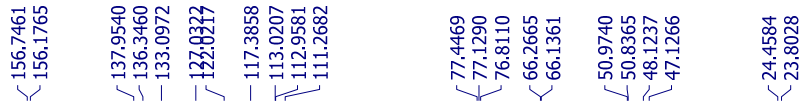

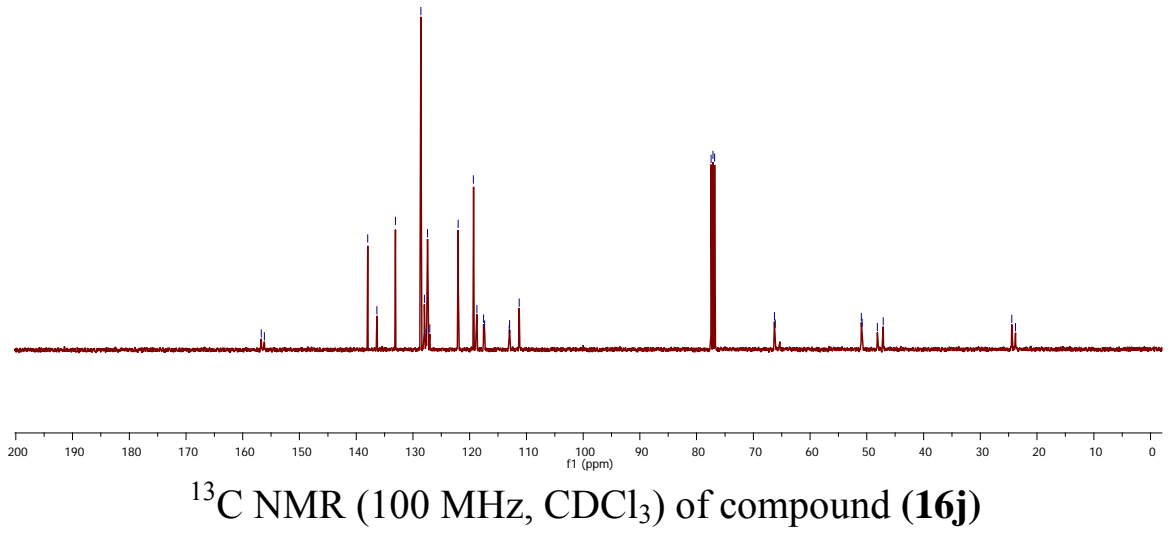




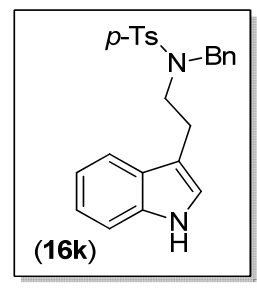

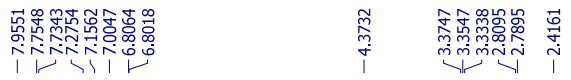

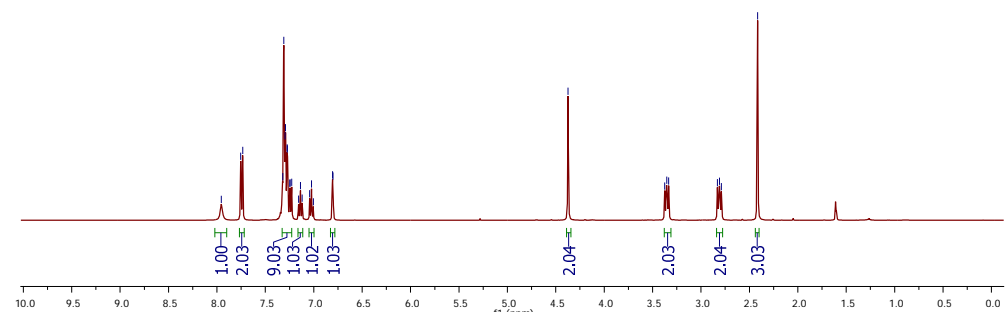

${ }^{1} \mathrm{H}$ NMR (400 MHz, $\mathrm{CDCl}_{3}$ ) of compound (16k)
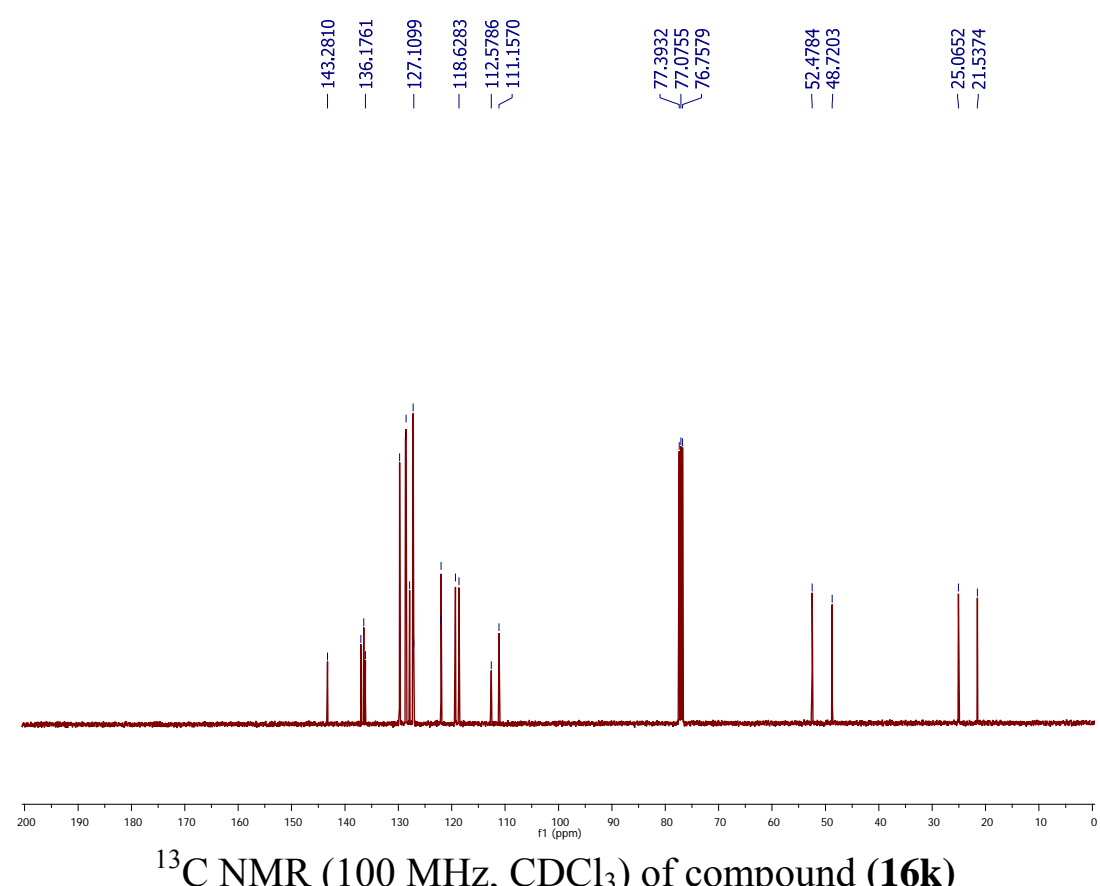

${ }^{13} \mathrm{C}$ NMR $\left(100 \mathrm{MHz}, \mathrm{CDCl}_{3}\right)$ of compound (16k) 


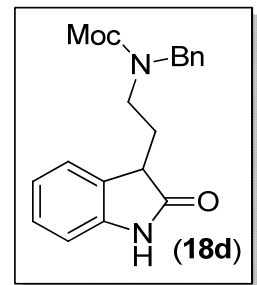

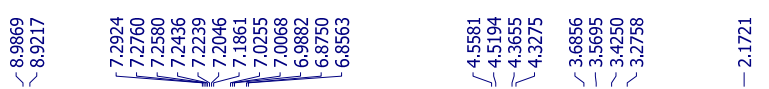
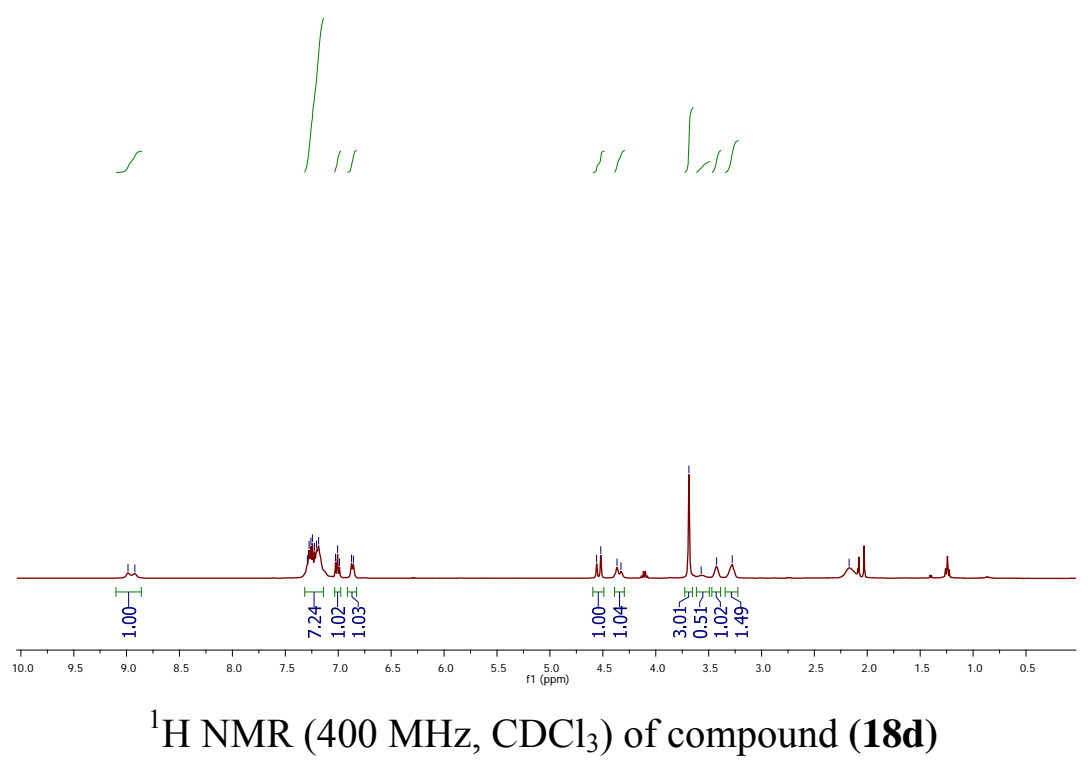

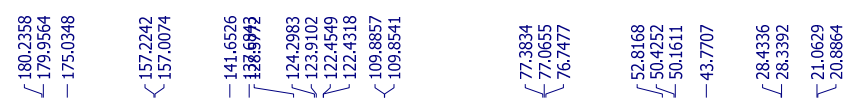

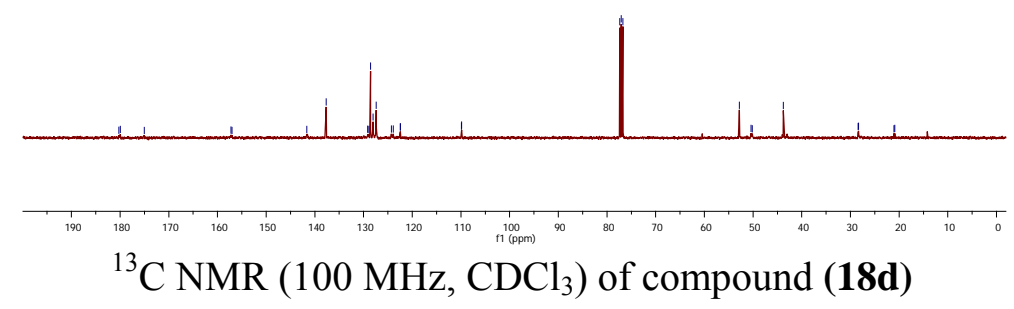




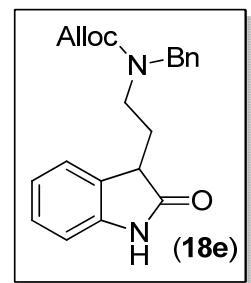

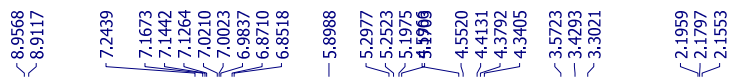

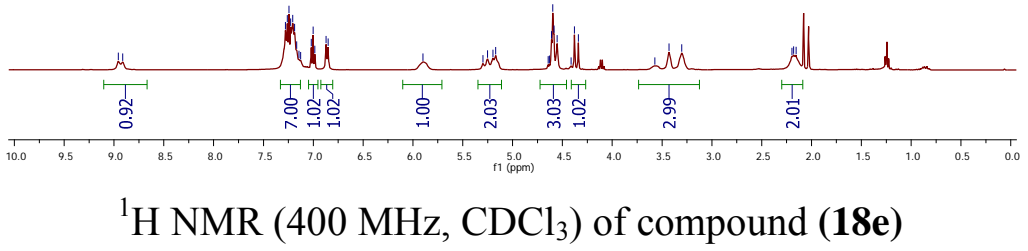

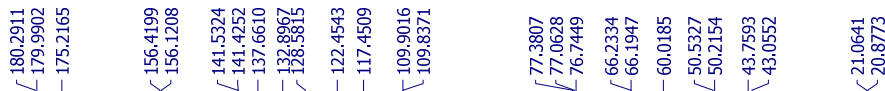
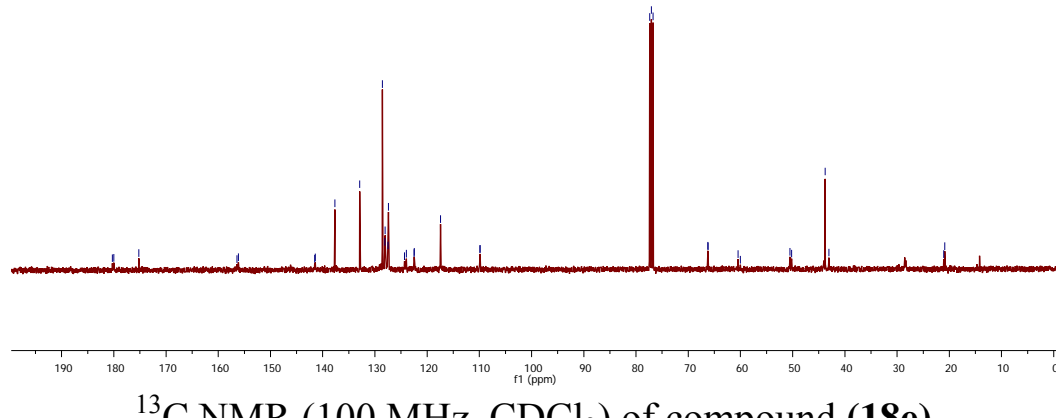

${ }^{13} \mathrm{C} \mathrm{NMR}\left(100 \mathrm{MHz}, \mathrm{CDCl}_{3}\right)$ of compound (18e) 


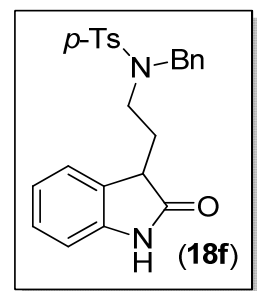

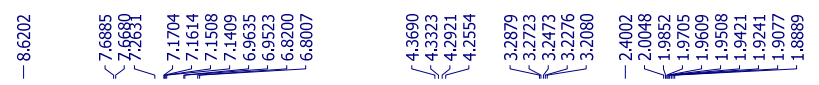

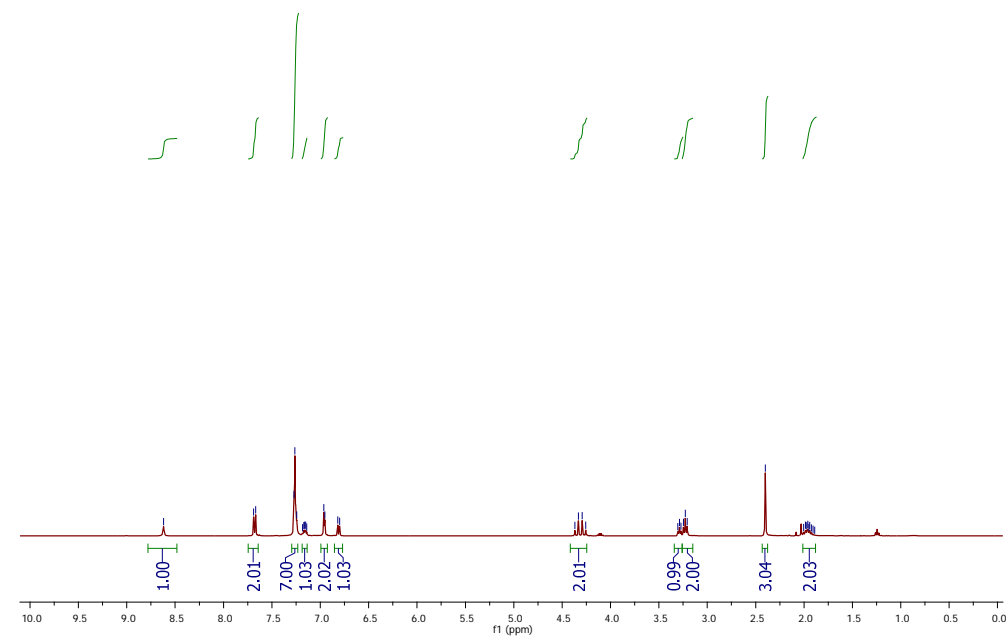

${ }^{1} \mathrm{H}$ NMR (400 MHz, $\mathrm{CDCl}_{3}$ ) of compound (18f)

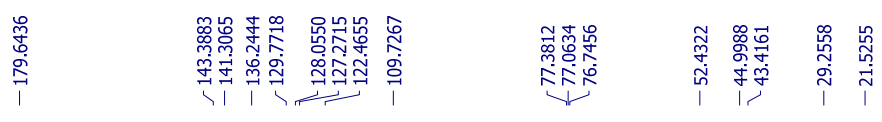
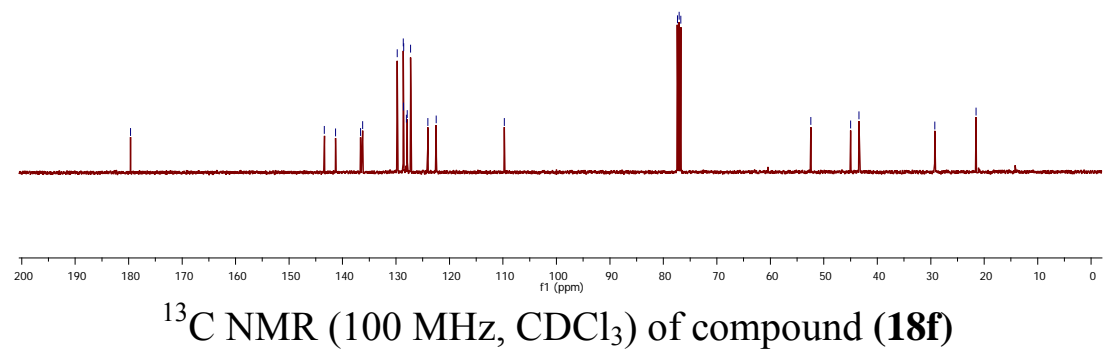

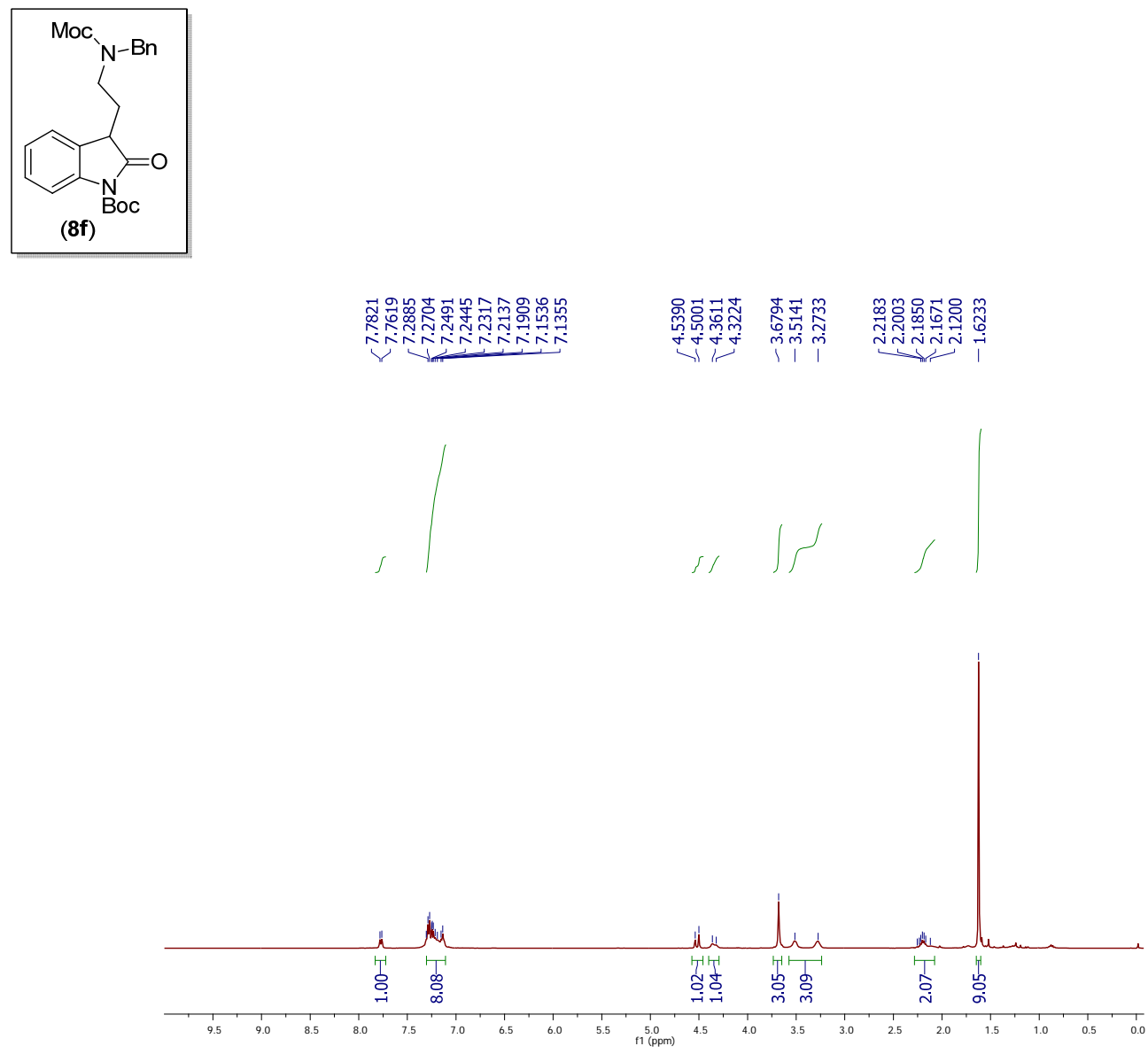

${ }^{1} \mathrm{H}$ NMR (400 MHz, $\mathrm{CDCl}_{3}$ ) of compound (8f)

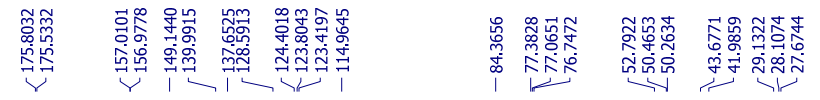
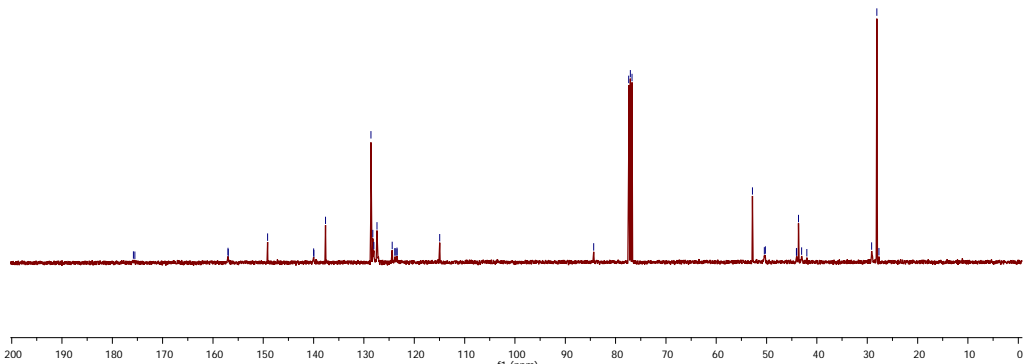

${ }^{13} \mathrm{C}$ NMR (100 MHz, $\mathrm{CDCl}_{3}$ ) of compound (8f) 


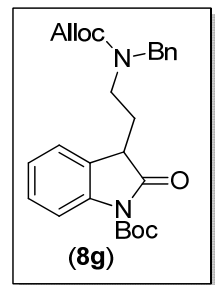

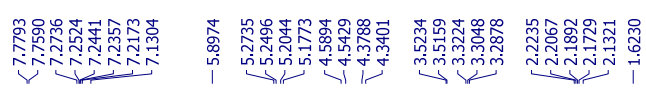

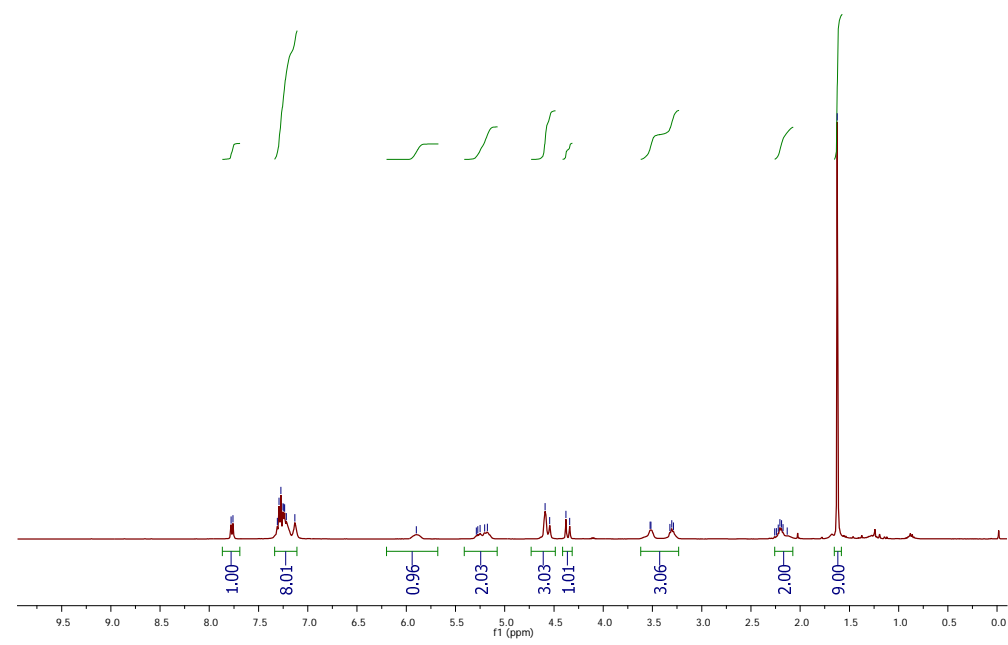

${ }^{1} \mathrm{H}$ NMR (400 MHz, $\mathrm{CDCl}_{3}$ ) of compound (8g)

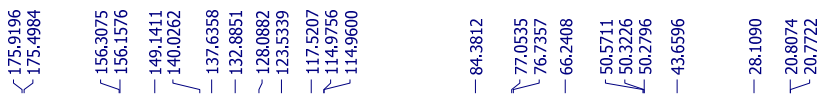
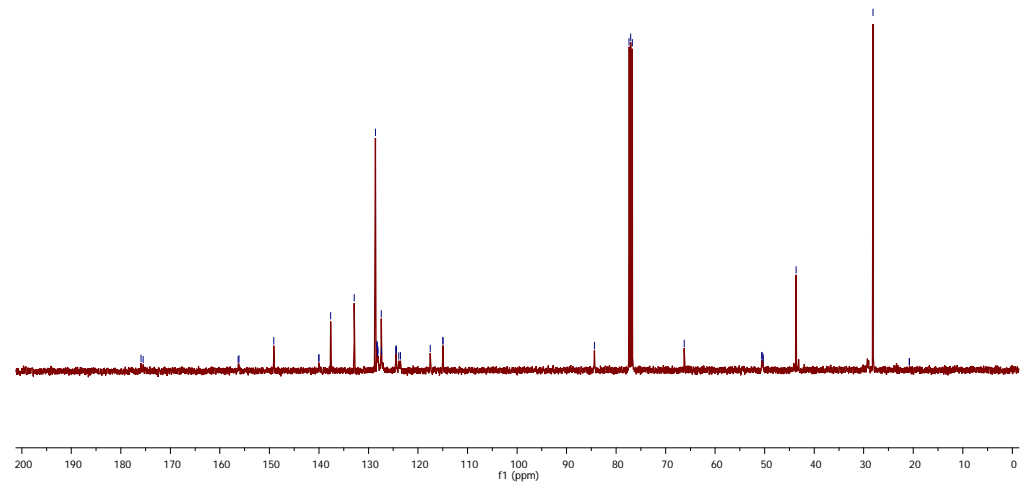

${ }^{13} \mathrm{C}$ NMR (100 MHz, $\left.\mathrm{CDCl}_{3}\right)$ of compound (8g) 


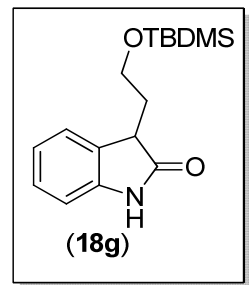

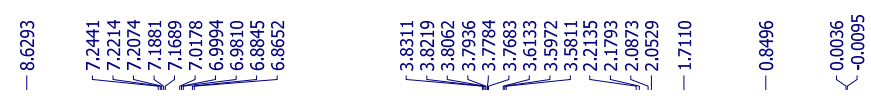

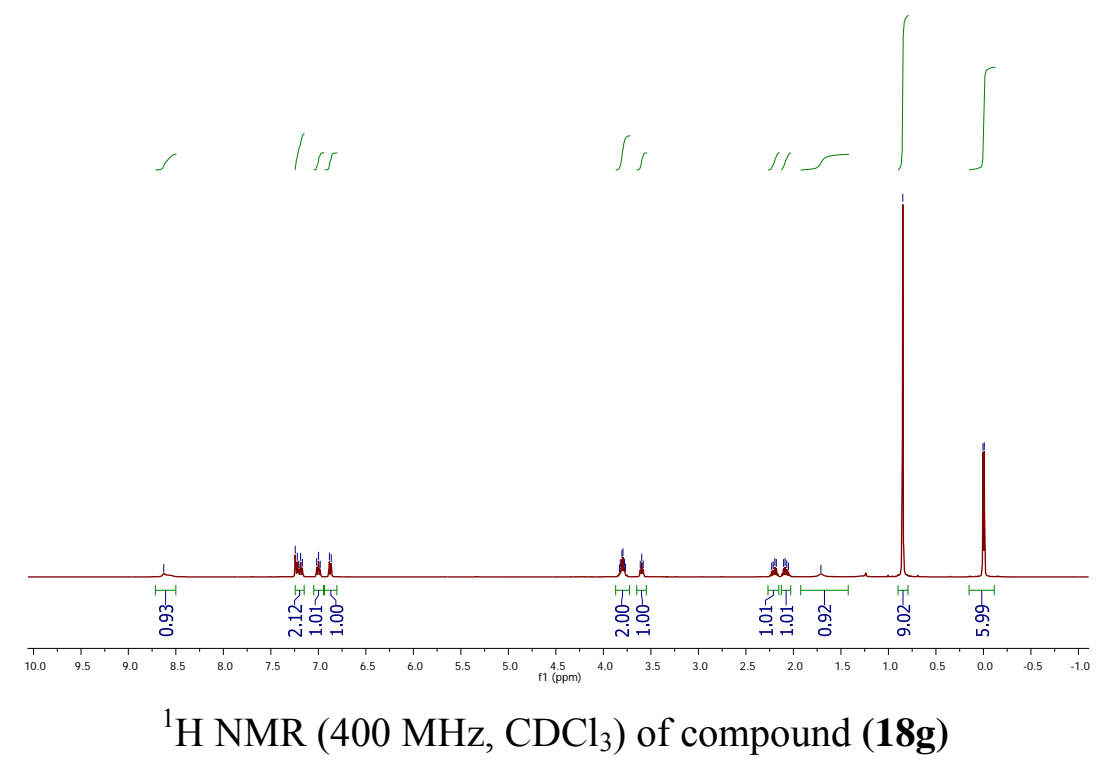



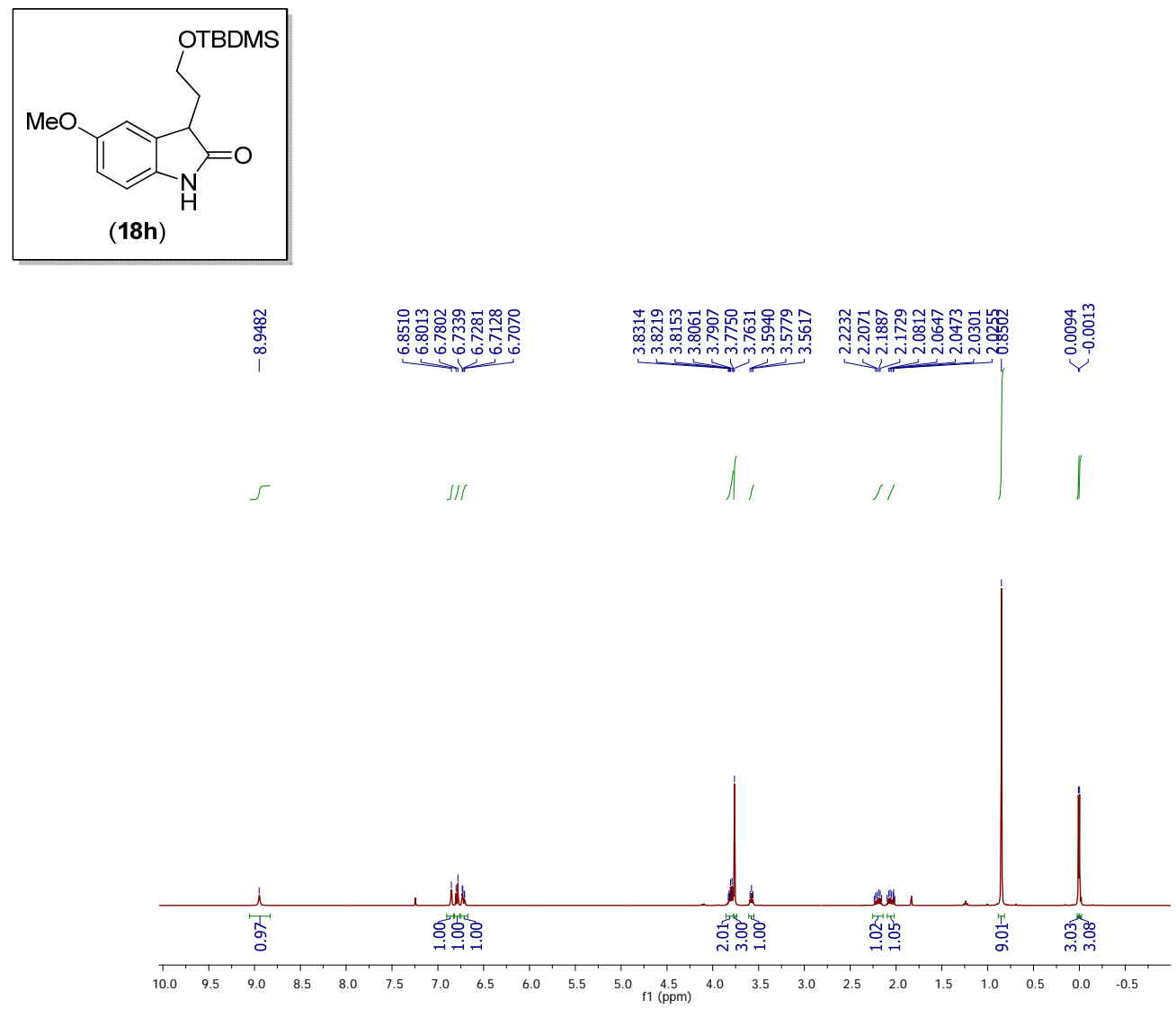

${ }^{1} \mathrm{H}$ NMR (400 MHz, $\mathrm{CDCl}_{3}$ ) of compound (18h)

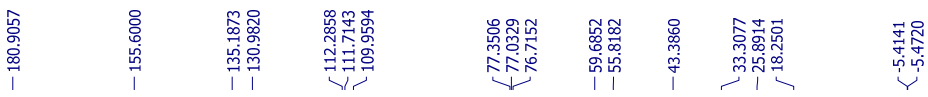

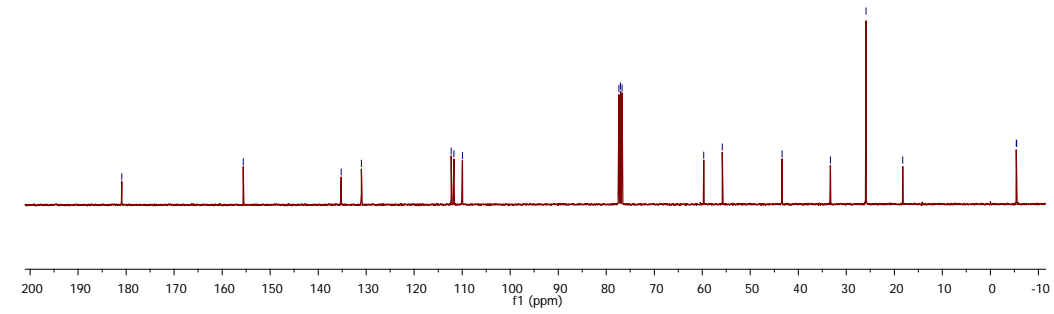

${ }^{13} \mathrm{C}$ NMR (100 MHz, $\left.\mathrm{CDCl}_{3}\right)$ of compound (18h) 


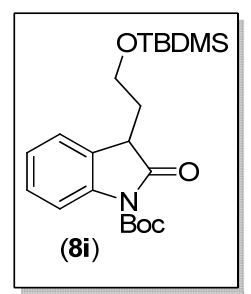

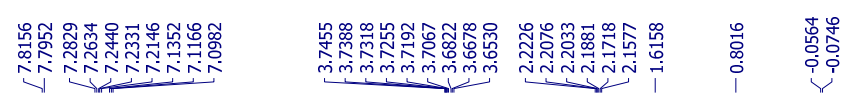
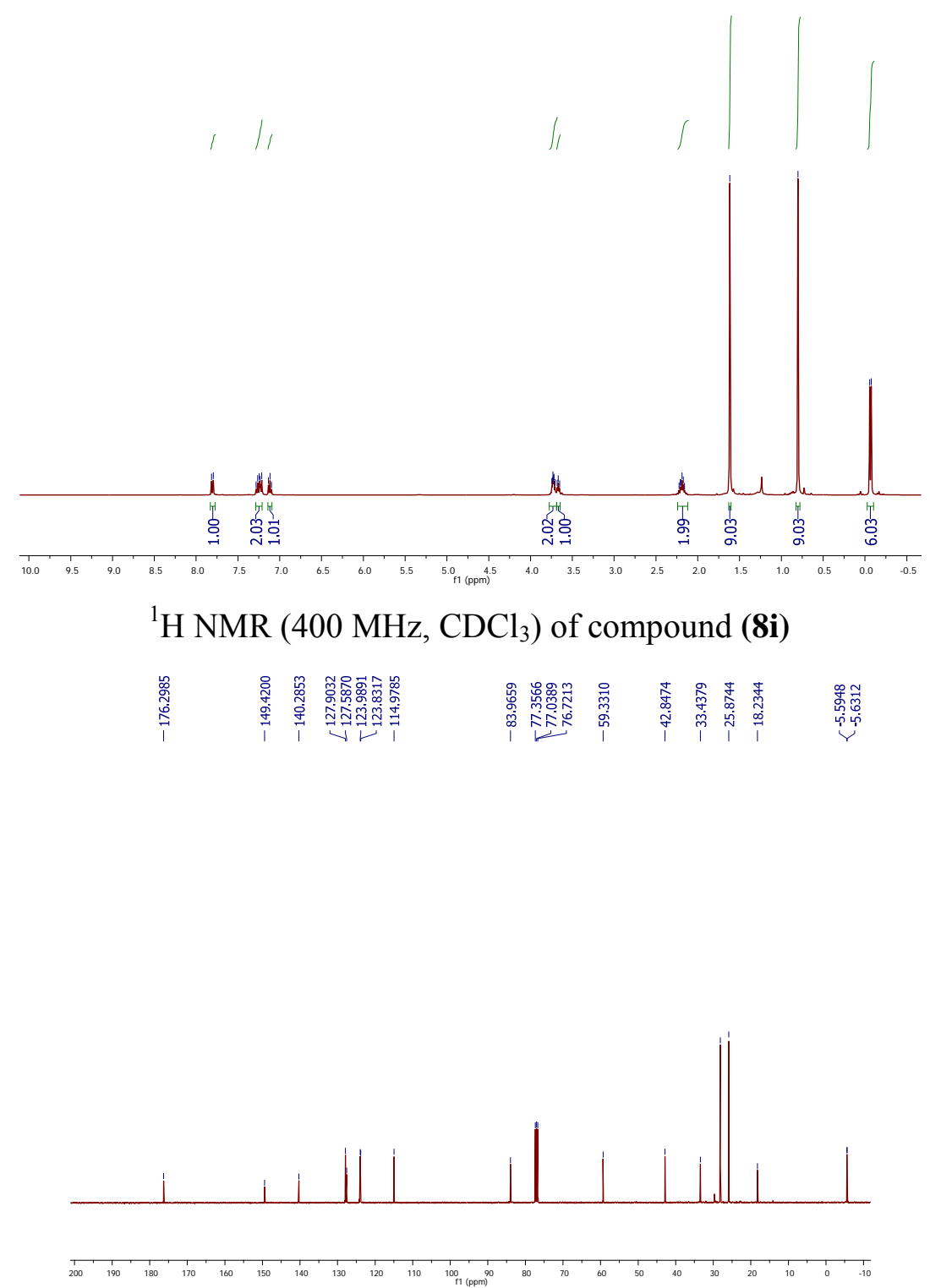

${ }^{13} \mathrm{C}$ NMR $\left(100 \mathrm{MHz}, \mathrm{CDCl}_{3}\right)$ of compound (8i) 

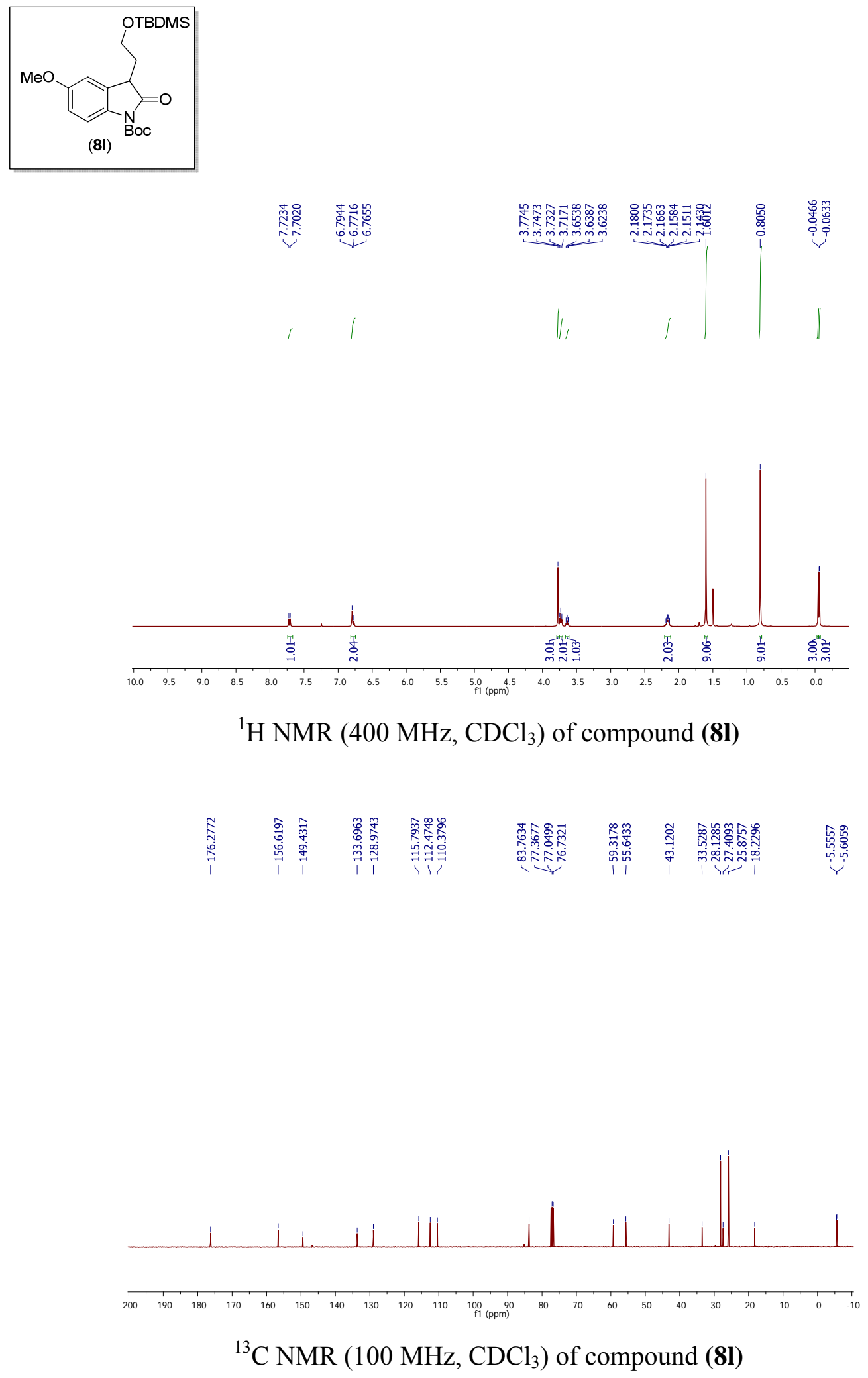

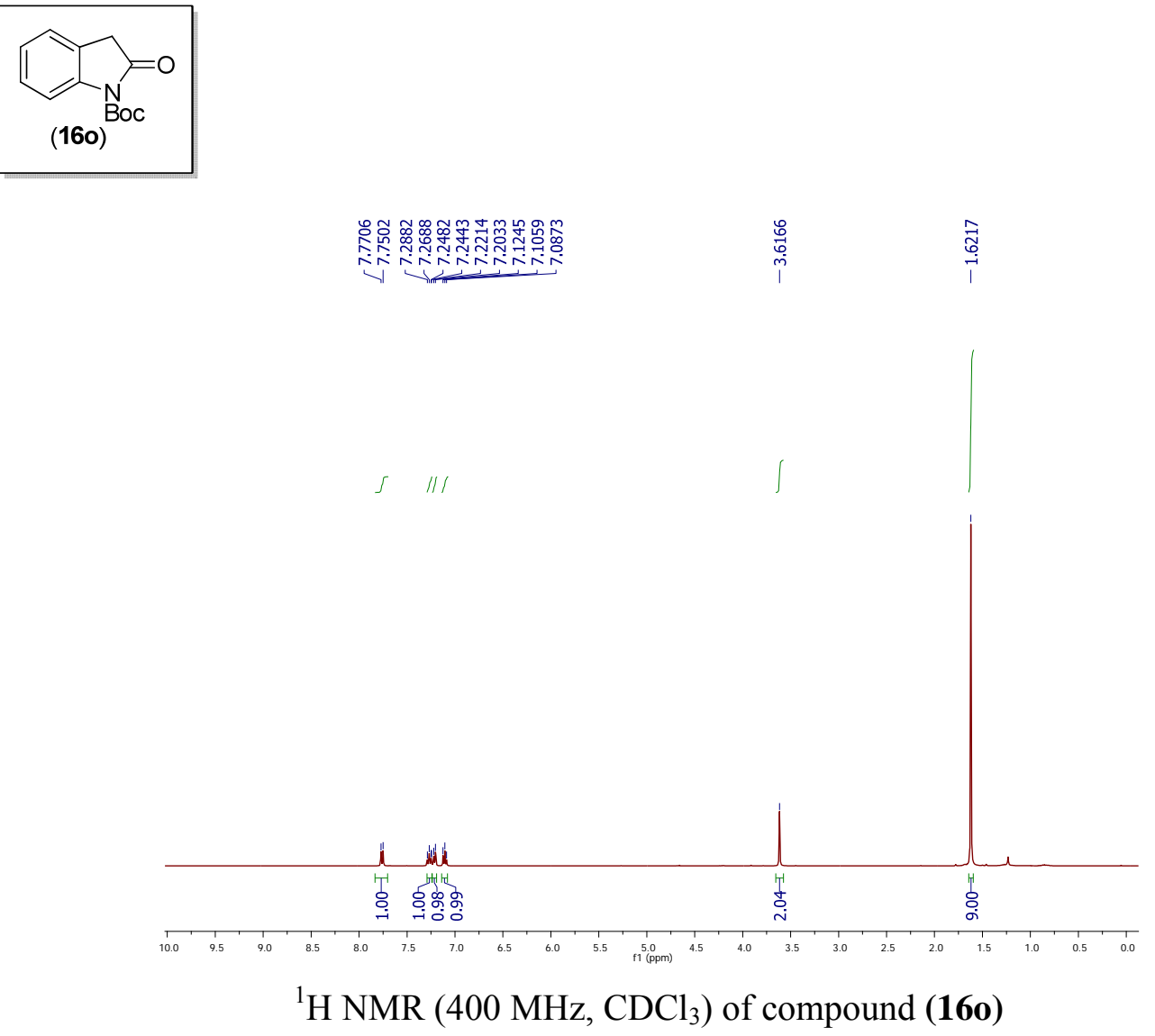

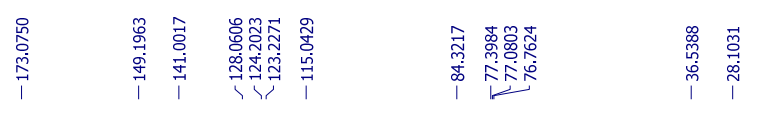

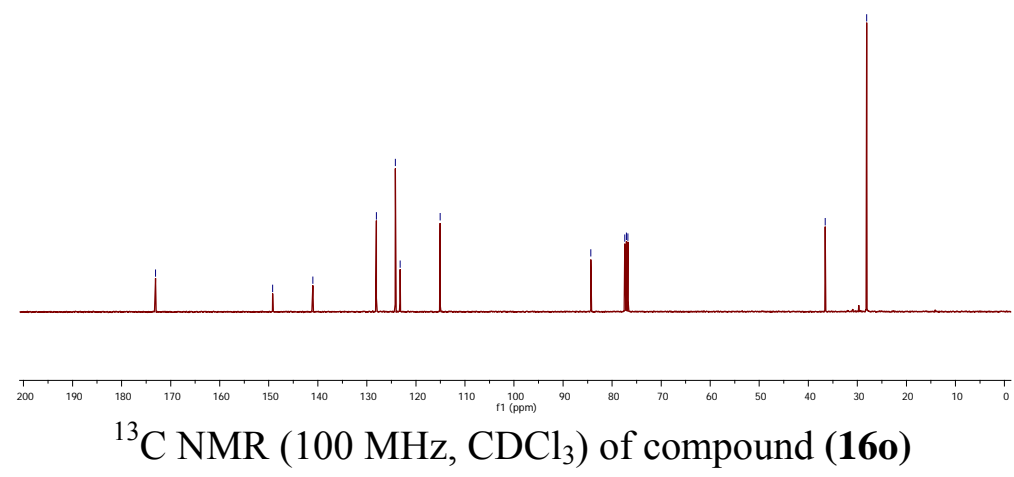




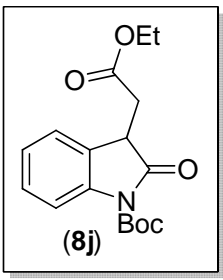

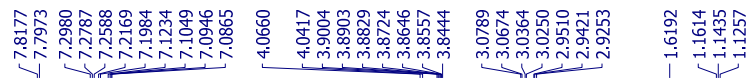

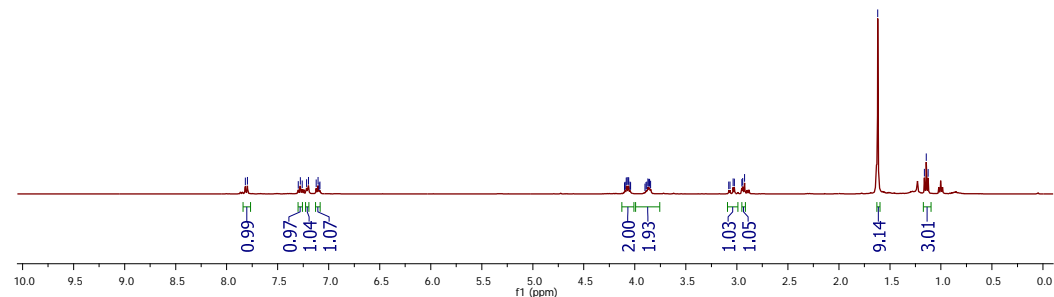

${ }^{1} \mathrm{H}$ NMR (400 MHz, $\mathrm{CDCl}_{3}$ ) of compound (8j)

H H H

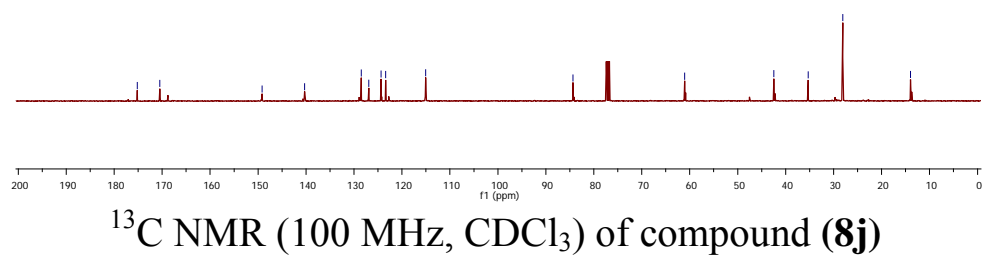




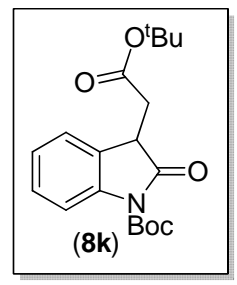

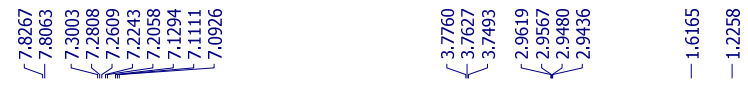

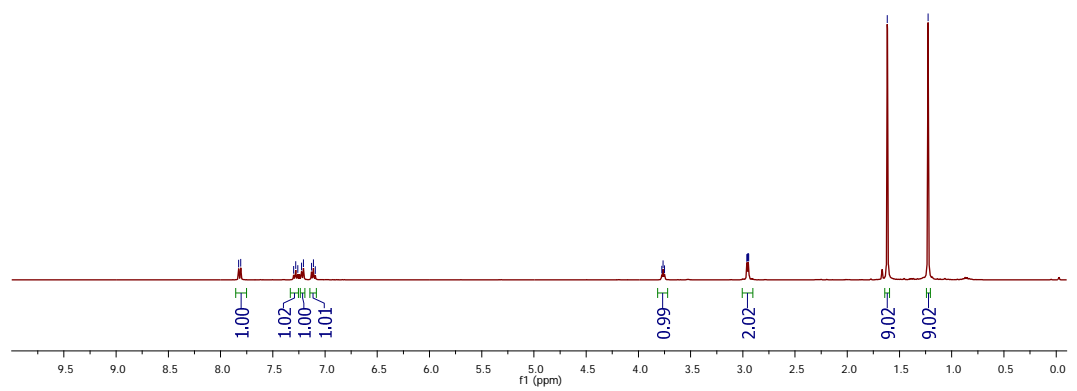

${ }^{1} \mathrm{H}$ NMR (400 MHz, $\mathrm{CDCl}_{3}$ ) of compound (8k)

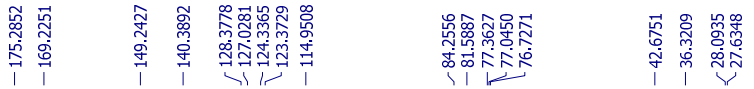

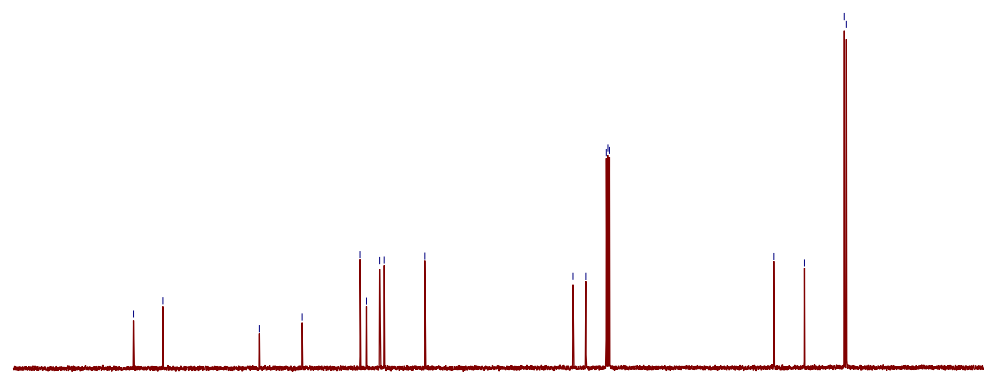

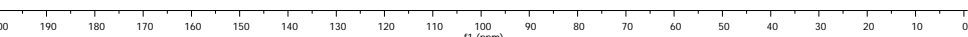

${ }^{13} \mathrm{C}$ NMR $\left(100 \mathrm{MHz}, \mathrm{CDCl}_{3}\right)$ of compound (8k) 


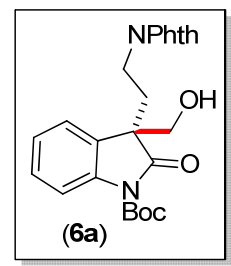

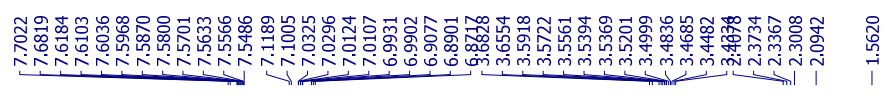
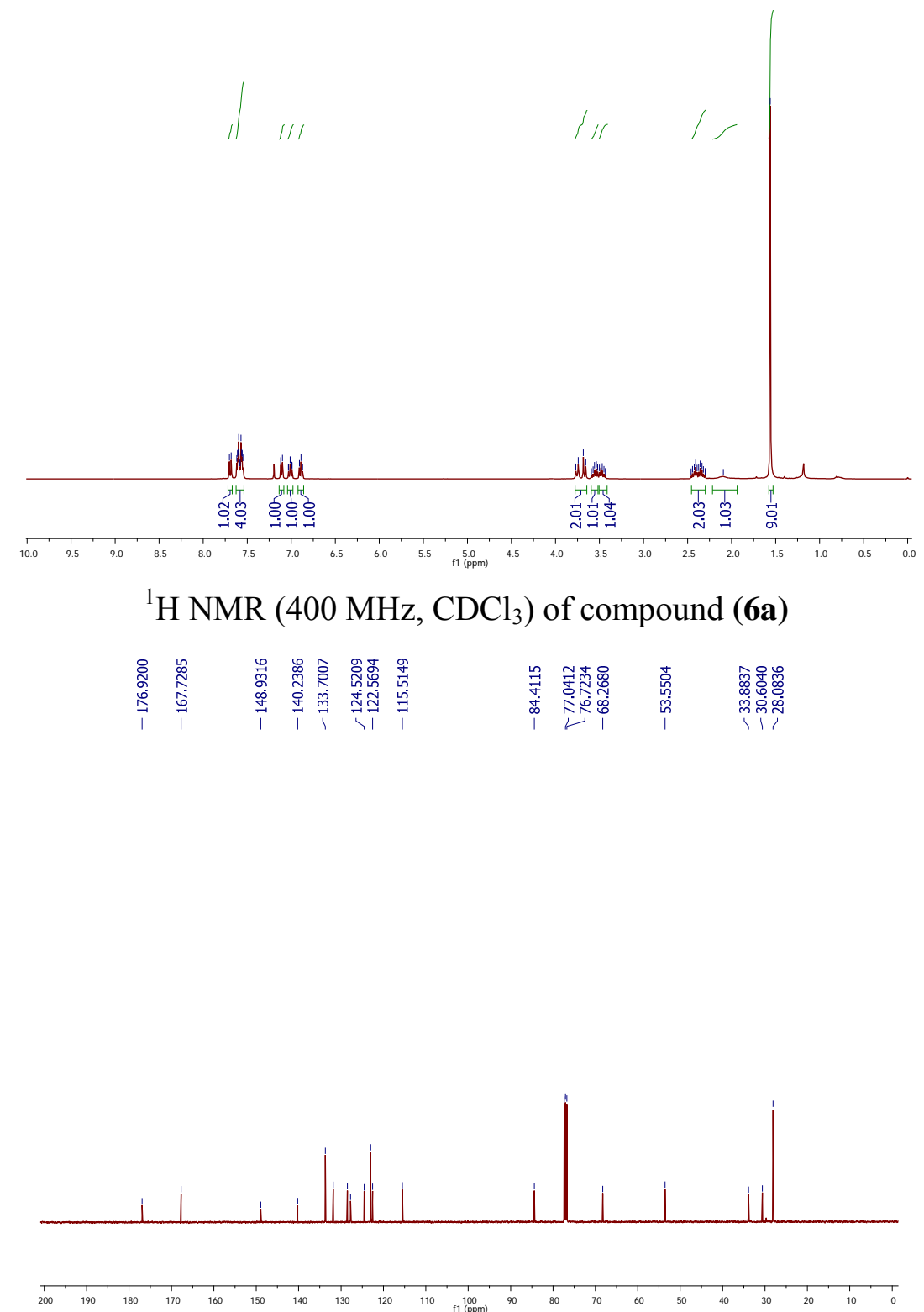

${ }^{13} \mathrm{C}$ NMR (100 MHz, $\mathrm{CDCl}_{3}$ ) of compound (6a) 


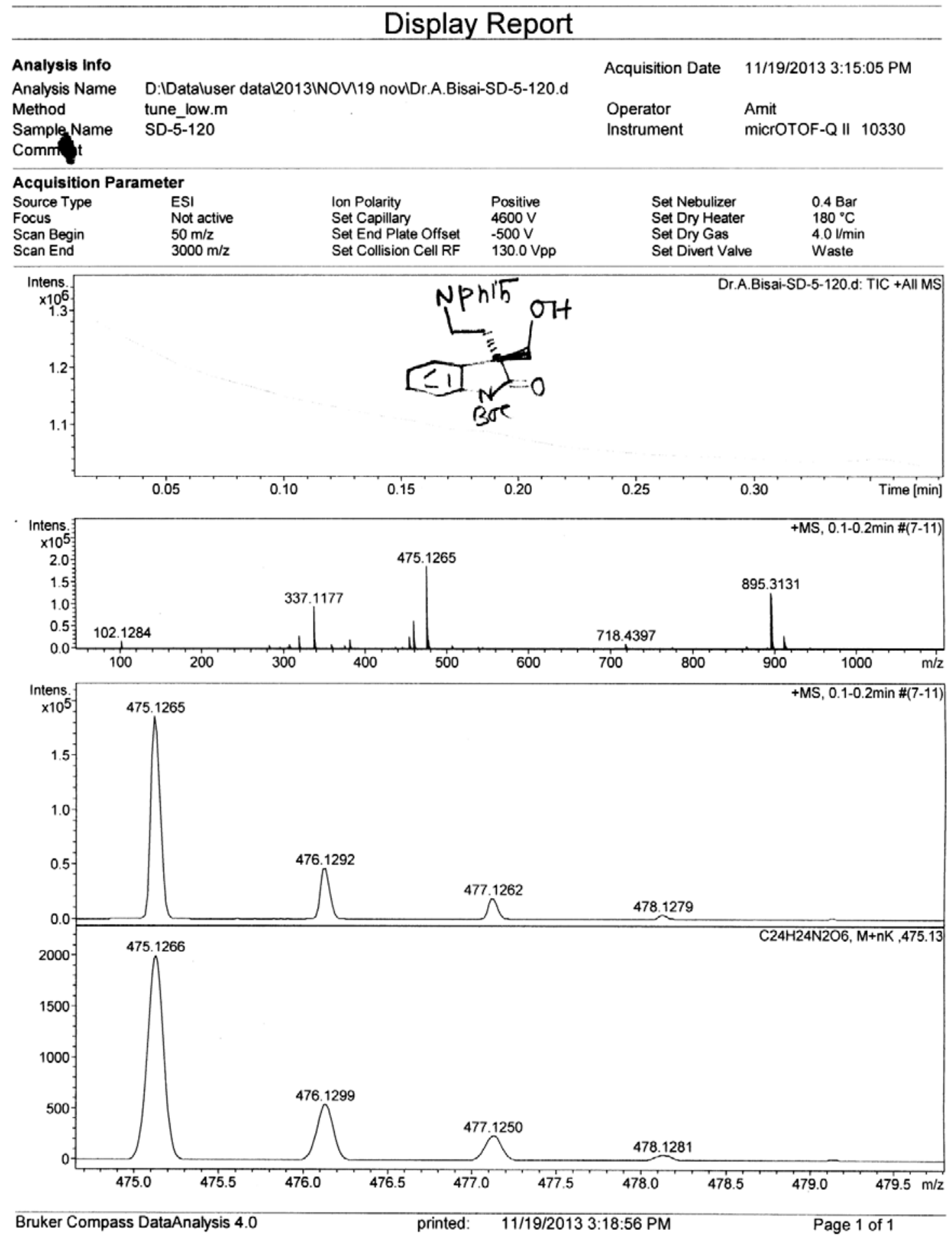

Scanned copy of mass spectrum of (6a) 


\section{HPLC data of ( \pm )-6a:}

Data File C: \CHEM32\1\DATA \SUBHADIP \2013-11-17AB-SD-5-120RACEMICF-IB-15-238-1-25.D Sample Name: AB-SD-5-120RACEMICF-IB-15-238-1-25

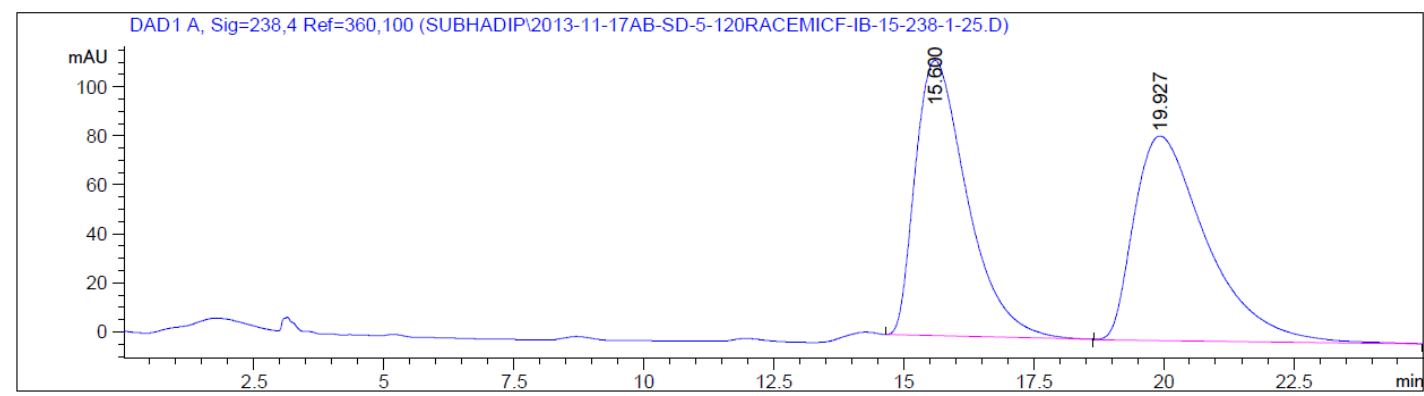

Signal 1: DAD1 A, Sig $=238,4$ Ref $=360,100$

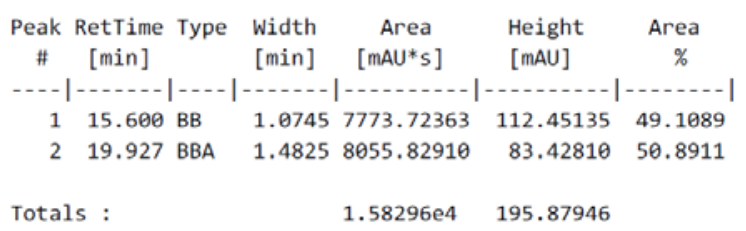

*** End of Report ***

\section{HPLC data of (+)-6a:}

Data File C: \CHEM32\1\DATA \SUBHADIP\2013-12-12SD-5-199-IB-15-238-25.D Sample Name: SD-5-199-IB-15-238-25

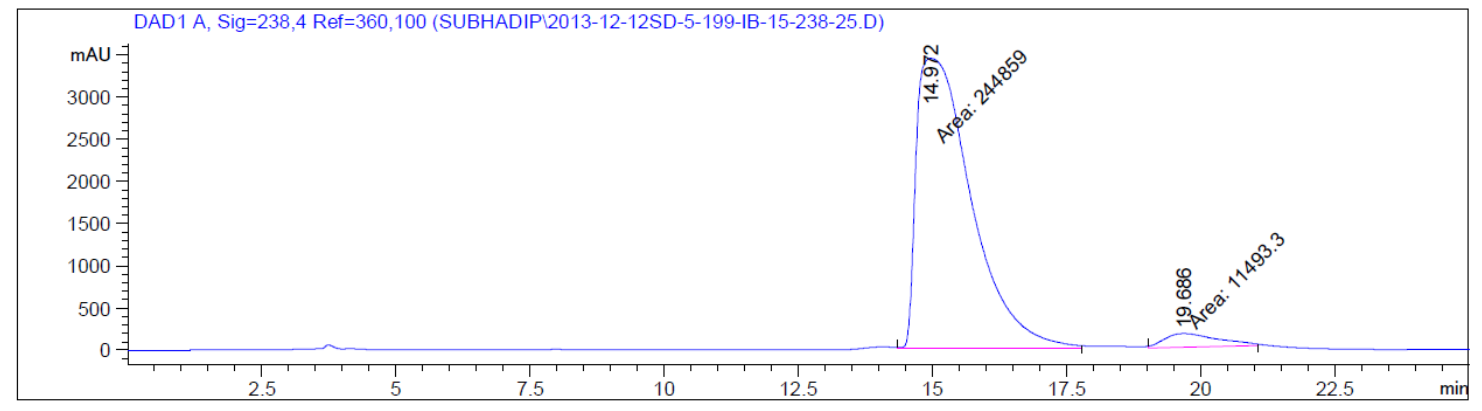

Signal 1: DAD1 A, Sig=238,4 Ref $=360,100$

\begin{tabular}{|c|c|c|c|c|c|c|}
\hline $\begin{array}{c}\text { Peak } \\
\#\end{array}$ & $\begin{array}{c}\text { RetTime } \\
\text { [min] }\end{array}$ & Type & $\begin{array}{l}\text { Width } \\
\text { [min] }\end{array}$ & $\begin{array}{c}\text { Area } \\
{\left[\mathrm{mAU}^{*} \mathrm{~s}\right]}\end{array}$ & $\begin{array}{l}\text { Height } \\
{[\mathrm{mAU}]}\end{array}$ & $\begin{array}{c}\text { Area } \\
\%\end{array}$ \\
\hline--1 & & & & | $\ldots \ldots$ & $|-\ldots-\ldots-n|$ & | \\
\hline 1 & 14.972 & MM & 1.1881 & $2.44859 \mathrm{e} 5$ & 3434.85986 & 95.5166 \\
\hline 2 & 19.686 & MM & 1.1716 & $1.14933 \mathrm{e} 4$ & 163.50507 & 4.4834 \\
\hline & & & & $2.56353 \mathrm{e} 5$ & 3598.36493 & \\
\hline
\end{tabular}




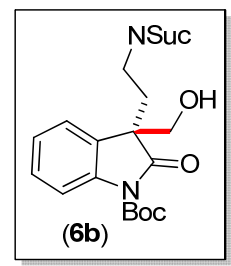

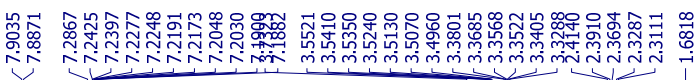
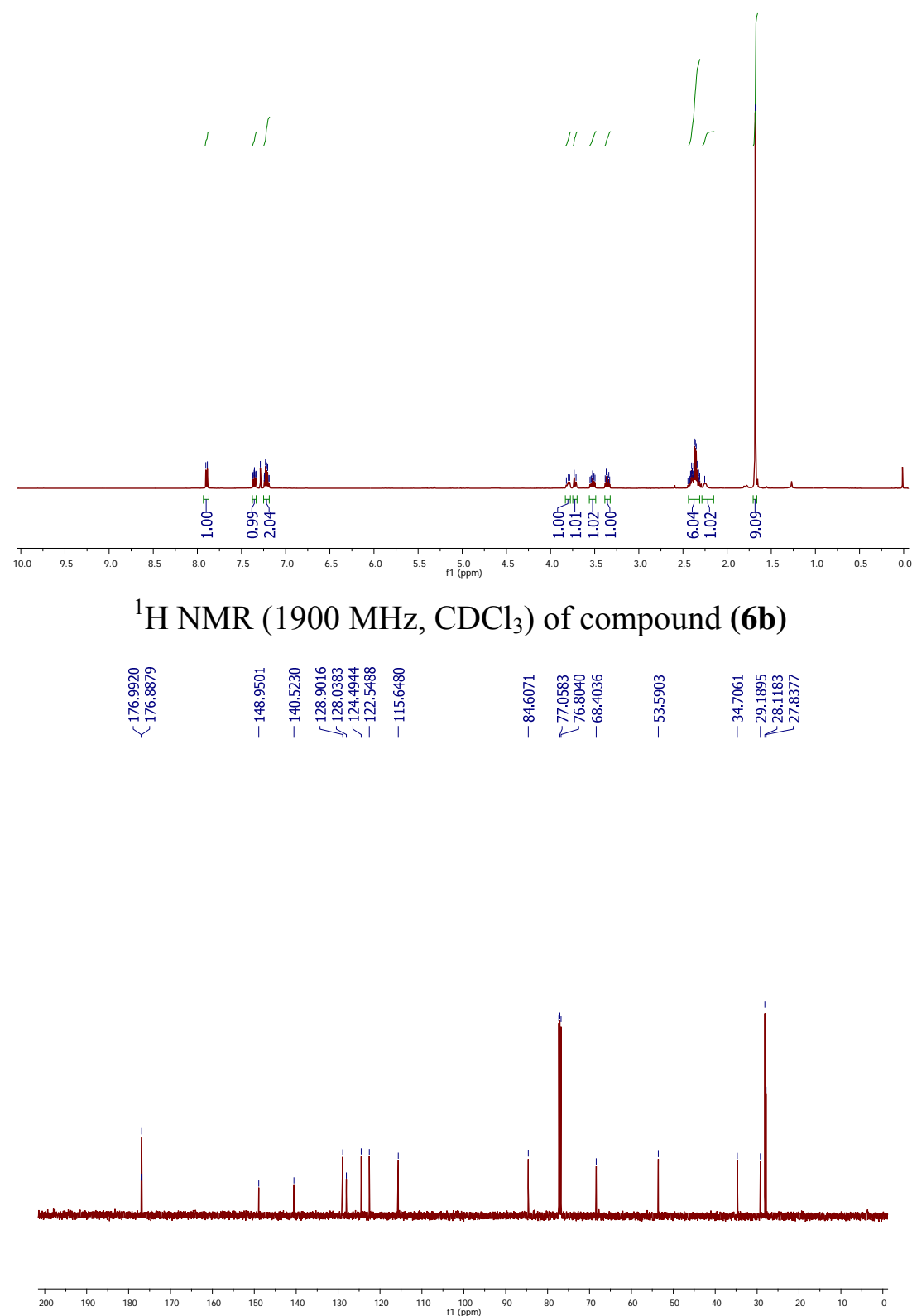

${ }^{13} \mathrm{C}$ NMR $\left(1219 \mathrm{MHz}, \mathrm{CDCl}_{3}\right)$ of compound (6b) 

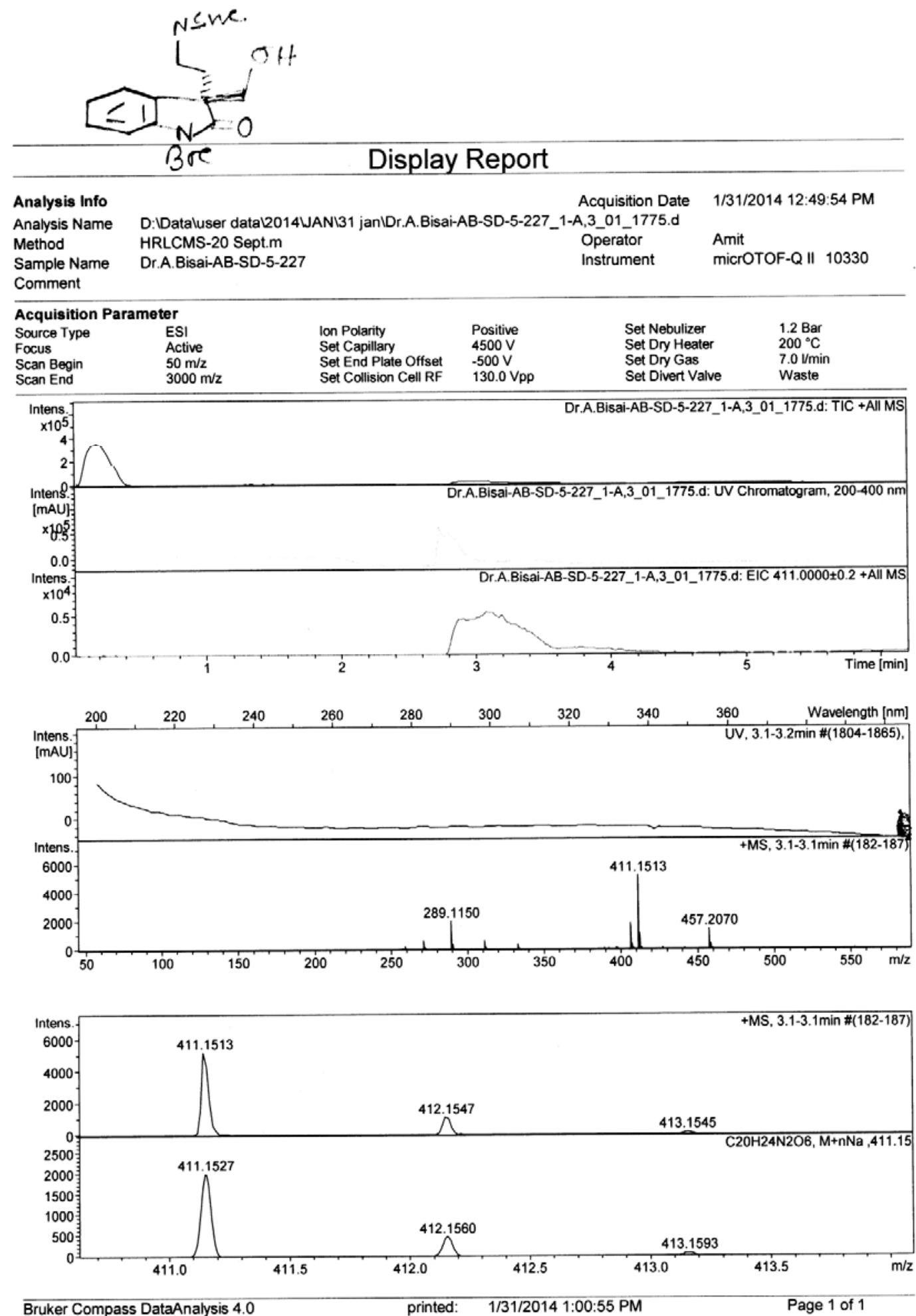

Scanned copy of mass spectrum of (6b) 


\section{HPLC data of ( \pm )-6b:}

Data File C: \CHEM32\1\DATA \SUBHADIP \2014-01-07SD-5-226-RACEMIC-IB-20-254-1-30.D Sample Name: SD-5-226-Racemic-IB-20-254-1-30

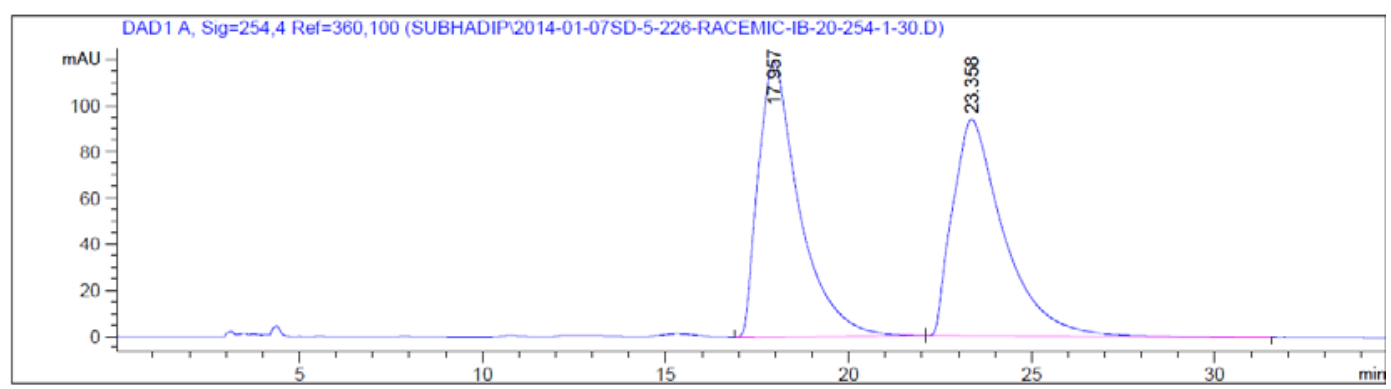

Signal 1: DAD1 A, Sig=254,4 Ref $=360,100$

\begin{tabular}{|c|c|c|c|c|c|c|}
\hline $\begin{array}{c}\text { Peak } \\
\#\end{array}$ & $\begin{array}{c}\text { RetTime } \\
\text { [min] }\end{array}$ & Type & $\begin{array}{l}\text { Width } \\
\text { [min] }\end{array}$ & $\begin{array}{c}\text { Area } \\
{\left[\mathrm{mAU}^{*} \mathrm{~s}\right]}\end{array}$ & $\begin{array}{l}\text { Height } \\
{[\mathrm{mAU}]}\end{array}$ & $\begin{array}{c}\text { Area } \\
\%\end{array}$ \\
\hline 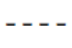 & & & & & & . \\
\hline 1 & 17.9 & BB & 4 & 9226.02637 & 119.01397 & 50.3371 \\
\hline 2 & 23.358 & BB & 687 & 9102.46289 & 93.75667 & 49.6629 \\
\hline
\end{tabular}

Totals : $\quad 1.83285 \mathrm{e} 4 \quad 212.77064$

$* * *$ End of Report $* * *$

\section{HPLC data of (-)-6b:}

Data File C: \CHEM32\1\DATA \SUBHADIP \2014-01-09SD-5-227-IB-20-254-1-40.D Sample Name: SD-5-227-IB-20-254-1-40

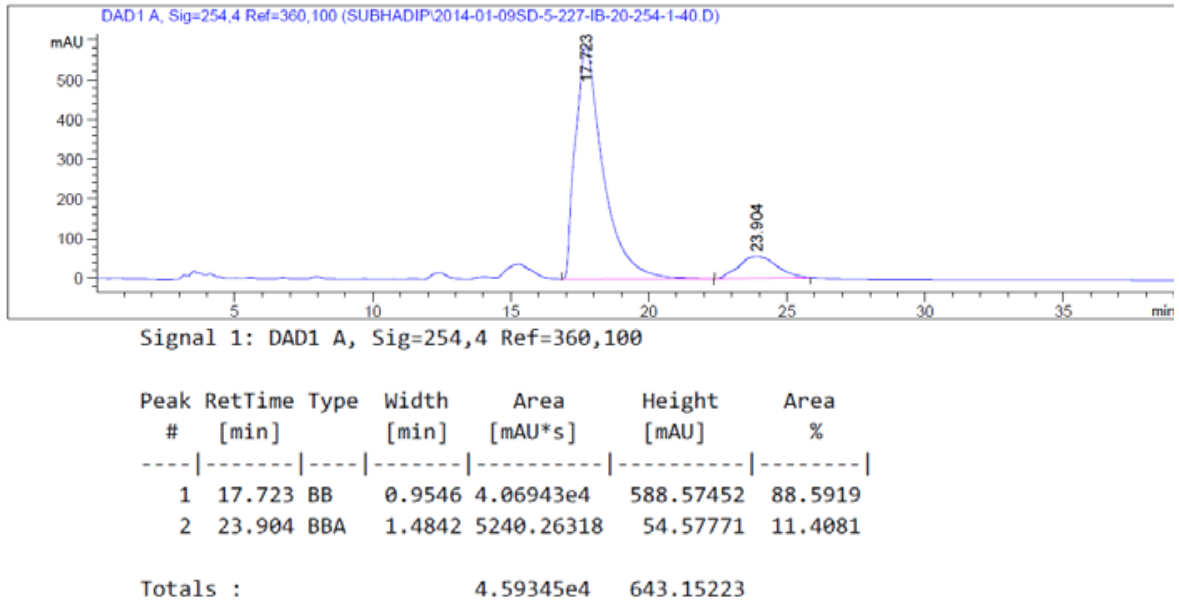

*** End of Report *** 


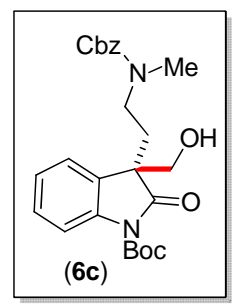

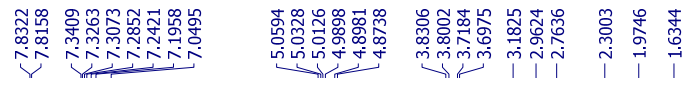

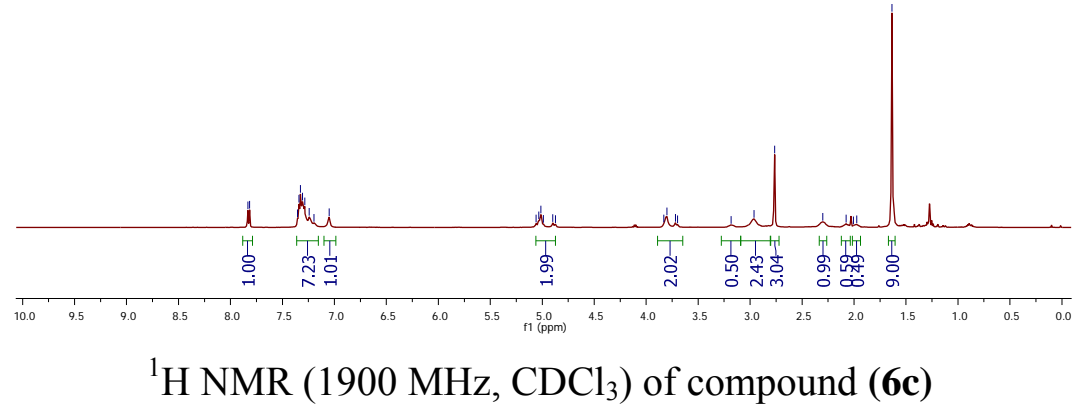

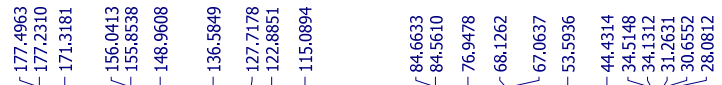

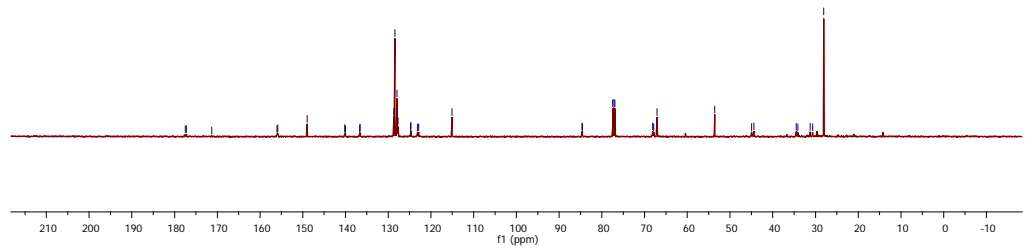

${ }^{13} \mathrm{C}$ NMR (1219 MHz, $\mathrm{CDCl}_{3}$ ) of compound (6c) 


\section{Display Report}
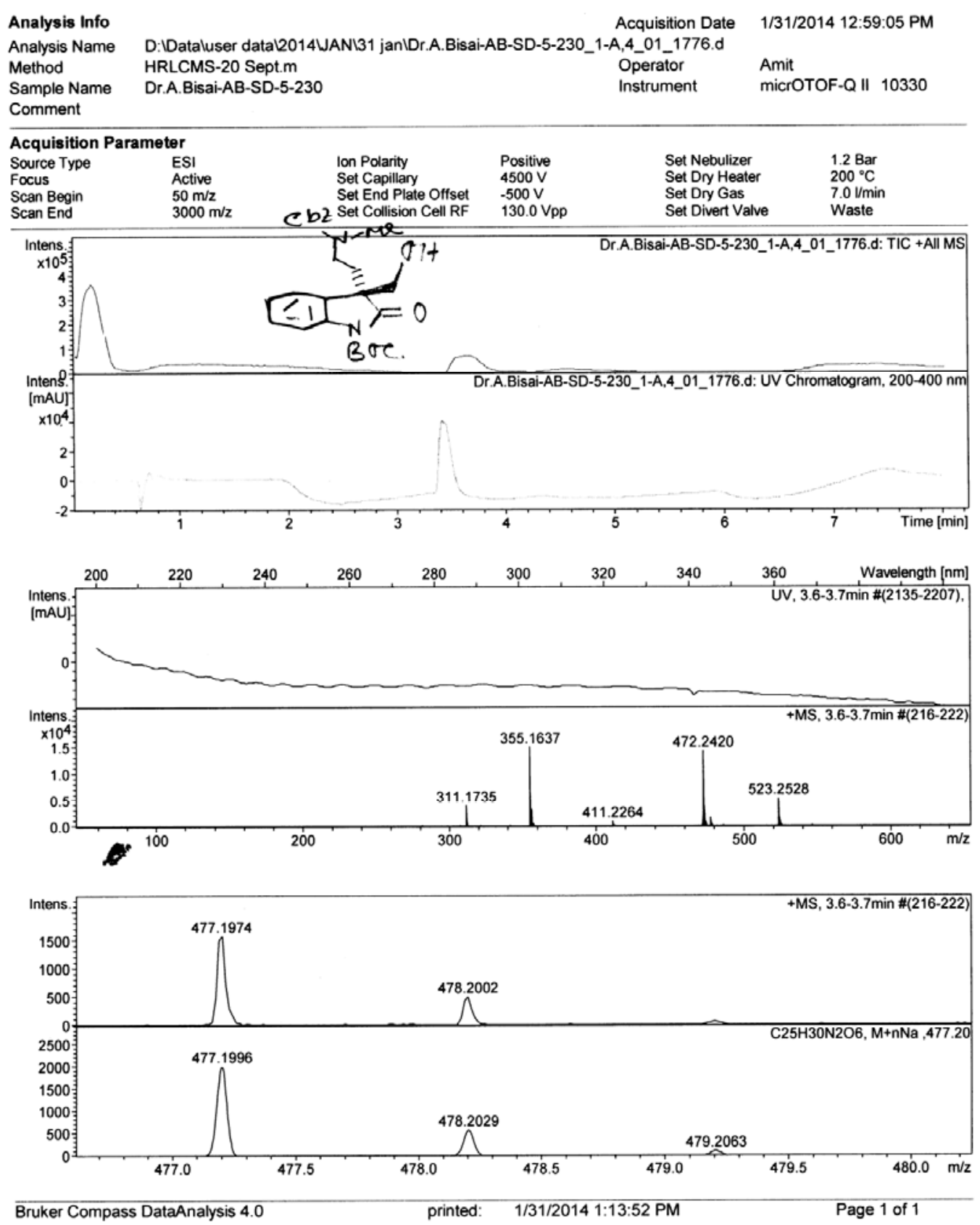

Scanned copy of mass spectrum of (6c) 


\section{HPLC data of ( \pm )-6c:}

Data File C: \CHEM32\1\DATA \SUBHADIP \2014-01-03SD-5-CBZ-RACEMIC-8-254-1-40.D

Sample Name: SD-5-Cbz-Racemic-8-254-1-40

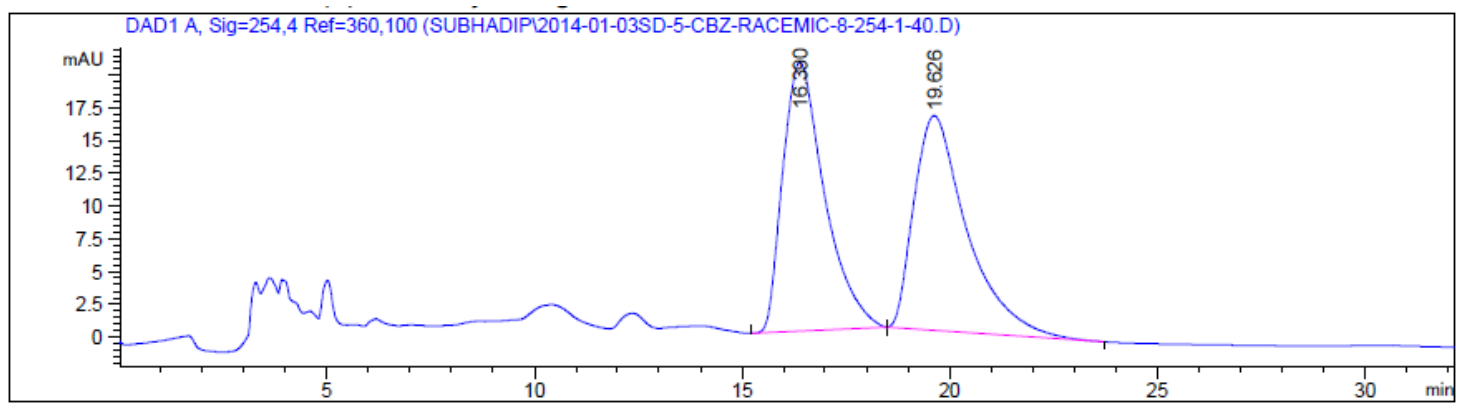

Signal 1: DAD1 A, Sig=254,4 Ref $=360,100$

\begin{tabular}{|c|c|c|c|c|c|c|}
\hline $\begin{array}{c}\text { Peak } \\
\#\end{array}$ & $\begin{array}{c}\text { RetTime } \\
\text { [min] }\end{array}$ & Type & $\begin{array}{l}\text { Width } \\
\text { [min] }\end{array}$ & $\begin{array}{c}\text { Area } \\
{\left[\mathrm{mAU}^{*} \mathrm{~s}\right]}\end{array}$ & $\begin{array}{l}\text { Height } \\
\text { [mAU] }\end{array}$ & $\begin{array}{c}\text { Area } \\
\%\end{array}$ \\
\hline . & & & & 1-- & -- & $---0 .-1$ \\
\hline 1 & 16.390 & BB & 1.0799 & 1437.79761 & 20.55893 & 49.6542 \\
\hline 2 & 19.626 & BB & 1.3521 & 1457.82275 & 16.41580 & 50.3458 \\
\hline Tota & : & & & 2895.62036 & 36.97473 & \\
\hline
\end{tabular}

\section{HPLC data of $(-)-6 c$ :}

Data File C: \CHEM32\1\DATA \SUBHADIP \2014-01-09SD-5-230-IB-8-254-1-40.D Sample Name: SD-5-230-IB-8-254-1-40

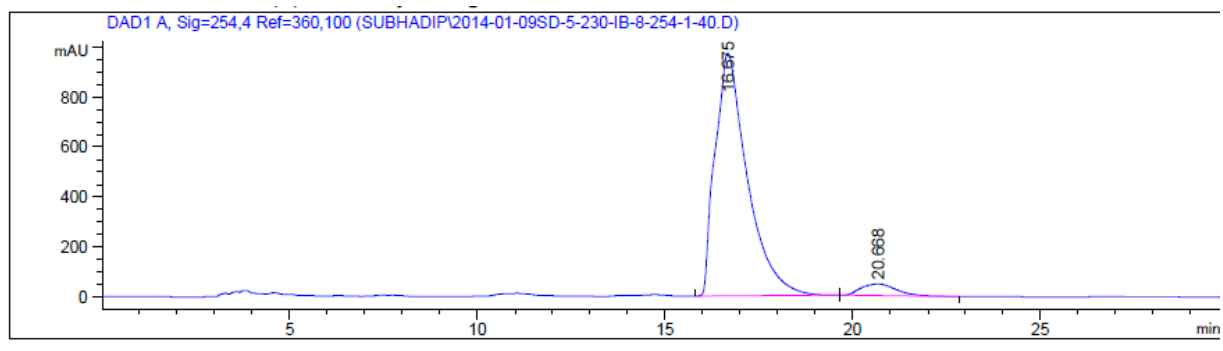

Signal 1: DAD1 A, Sig=254,4 Ref $=360,100$

\begin{tabular}{|c|c|c|c|c|c|c|}
\hline $\begin{array}{c}\text { Peak } \\
\#\end{array}$ & $\begin{array}{l}\text { RetTime } \\
\text { [min] }\end{array}$ & Type & $\begin{array}{l}\text { Width } \\
\text { [min] }\end{array}$ & $\begin{array}{c}\text { Area } \\
{\left[\mathrm{mAU}^{*} \mathrm{~s}\right]}\end{array}$ & $\begin{array}{l}\text { Height } \\
{[\mathrm{mAU}]}\end{array}$ & $\begin{array}{c}\text { Area } \\
\%\end{array}$ \\
\hline 1 & 16.675 & BR $>$ & a 8233 & 5.7904704 & 0715020 & 94.9322 \\
\hline 2 & 20.668 & BB & 1.0562 & 3091.17358 & 47.19347 & 5.0678 \\
\hline Tota] & Is: & & & $6.09959 \mathrm{e} 4$ & 1018.78569 & \\
\hline
\end{tabular}

*** End of Report *** 

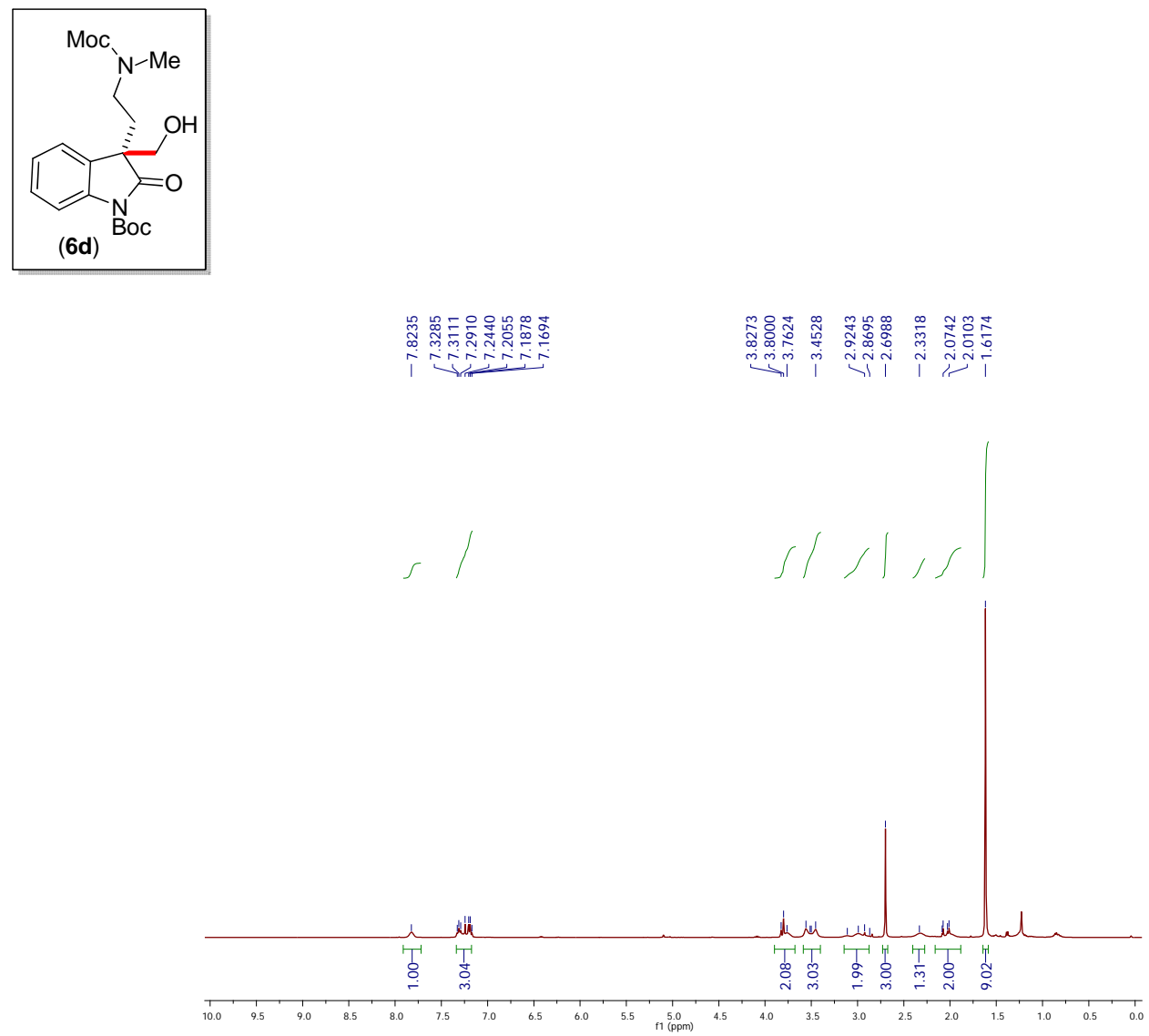

${ }^{1} \mathrm{H}$ NMR (400 MHz, $\mathrm{CDCl}_{3}$ ) of compound (6d)

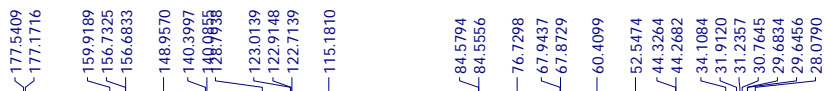
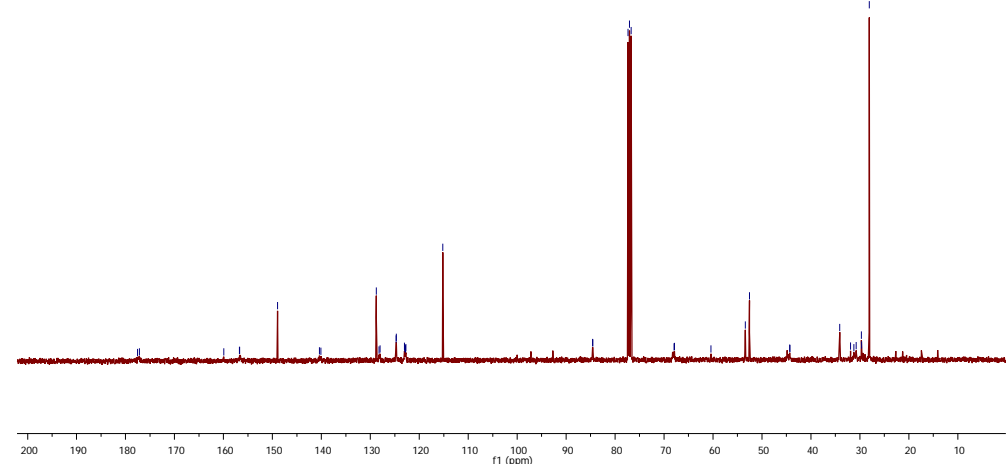

${ }^{13} \mathrm{C}$ NMR $\left(100 \mathrm{MHz}, \mathrm{CDCl}_{3}\right)$ of compound (6d) 


\section{Display Report}
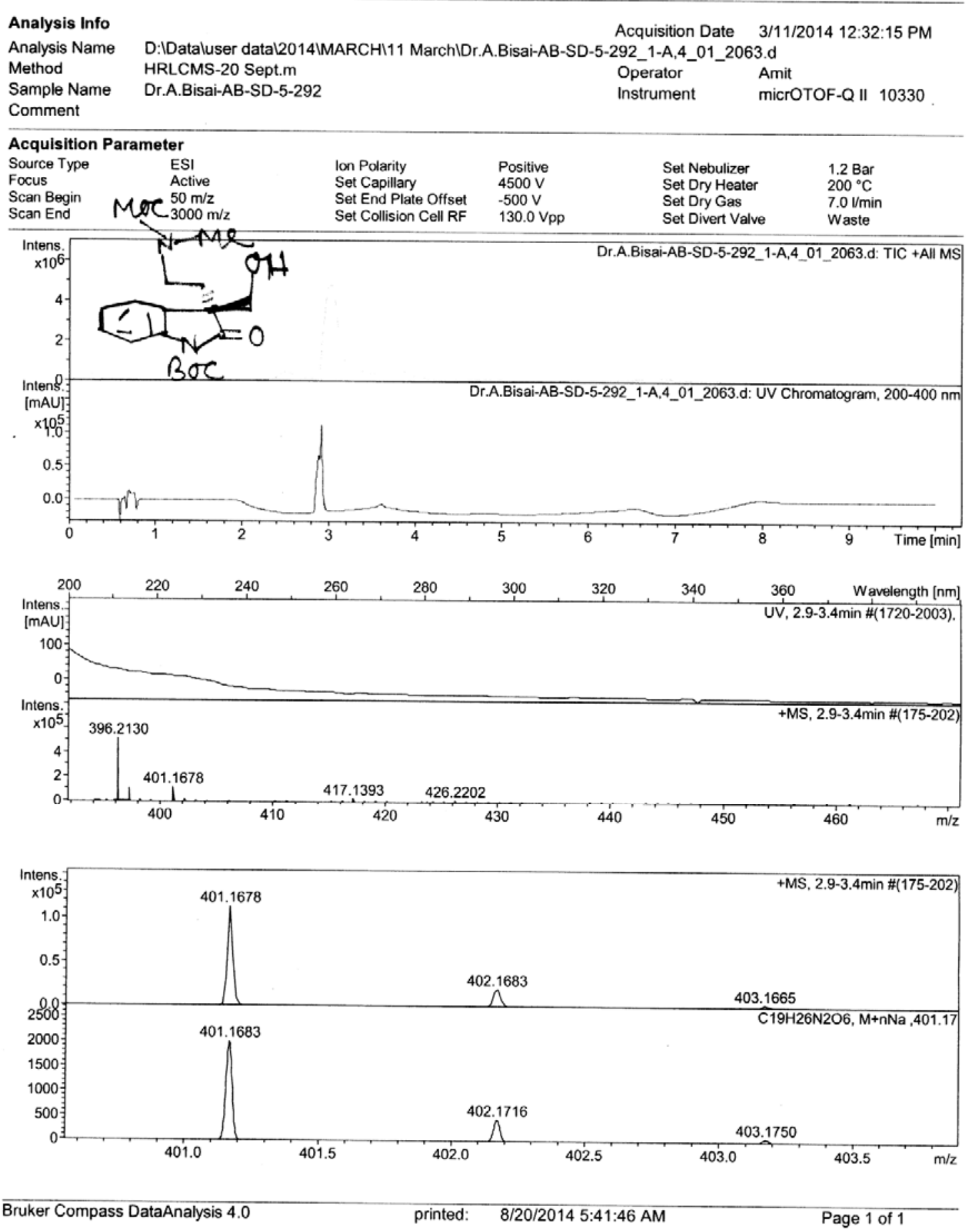

Scanned copy of mass spectrum of (6d) 


\section{HPLC data of ( \pm )-6d:}

Data File C: \CHEM32\1\DATA \SUBHADIP \2014-02-13SD-5-292-IB-10-254-1-60.D Sample Name: SD-5-292-IB-10-254-1-60

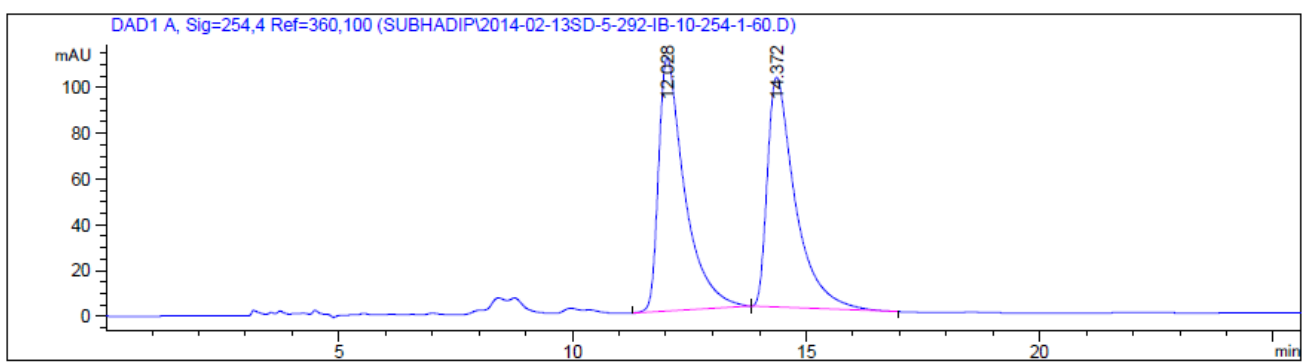

Signal 1: DAD1 A, Sig=254,4 Ref $=360,100$

\begin{tabular}{cccccc}
$\begin{array}{c}\text { Peak RetTime Type } \\
\text { \# } \\
\text { [min] }\end{array}$ & $\begin{array}{c}\text { Width } \\
{[\mathrm{min}]}\end{array}$ & $\begin{array}{c}\text { Area } \\
{[\mathrm{mAU} \text { s }]}\end{array}$ & $\begin{array}{c}\text { Height } \\
{[\mathrm{mAU}]}\end{array}$ & $\begin{array}{c}\text { Area } \\
\%\end{array}$ \\
\hline 1 & 12.028 BB & 0.5665 & 4307.11475 & 110.55067 & 50.7763 \\
2 & 14.372 BB & 0.6134 & 4175.41943 & 100.36495 & 49.2237
\end{tabular}

Totals : $\quad 8482.53418 \quad 210.91563$

\section{HPLC data of (-)-6d:}

Data File C: \CHEM32\1\DATA \SUBHADIP \2014-02-21SD-5-322R-IB-10-254-1-60.D Sample Name: SD-5-322R-IB-10-254-1-60

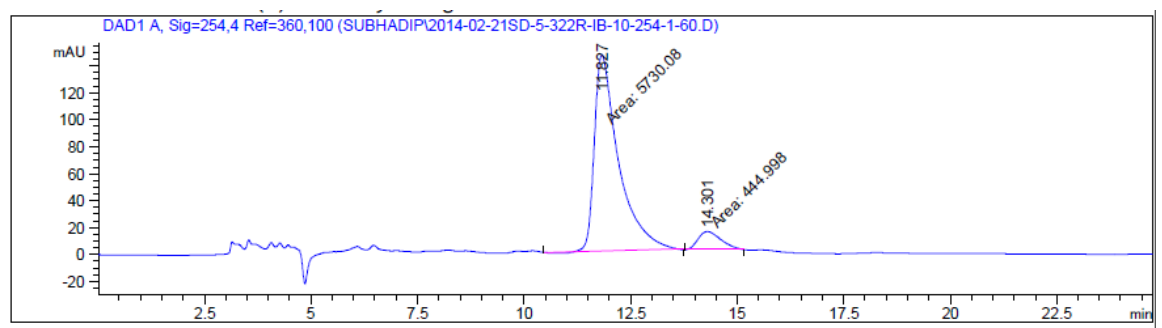

Signal 1: DAD1 A, Sig=254,4 Ref $=360,100$

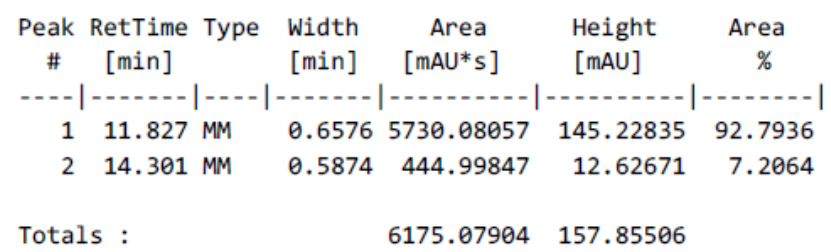

*** End of Report *** 


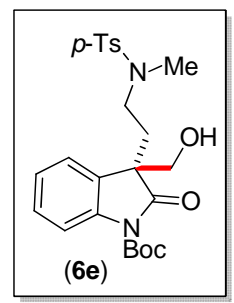

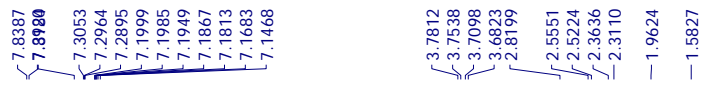

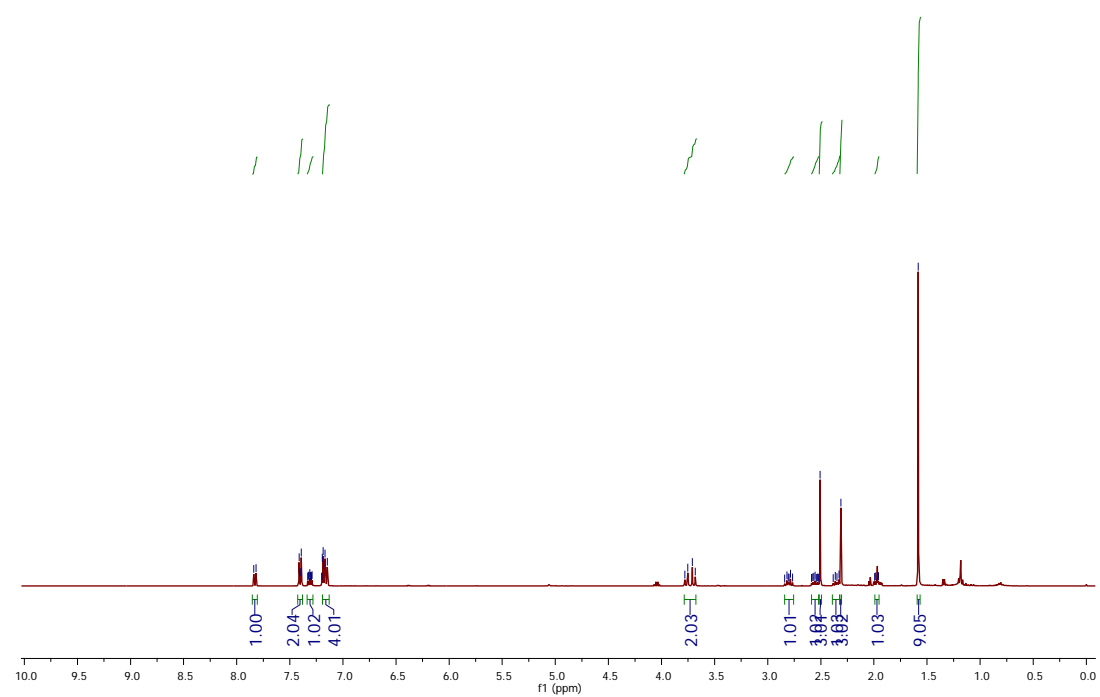

${ }^{1} \mathrm{H}$ NMR (400 MHz, $\mathrm{CDCl}_{3}$ ) of compound (6e)

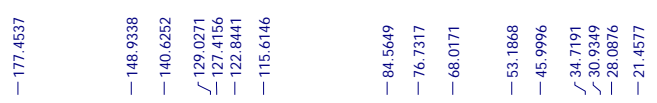

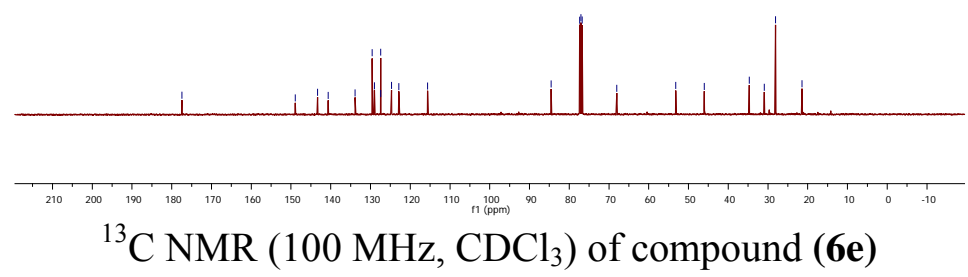




\section{Display Report}
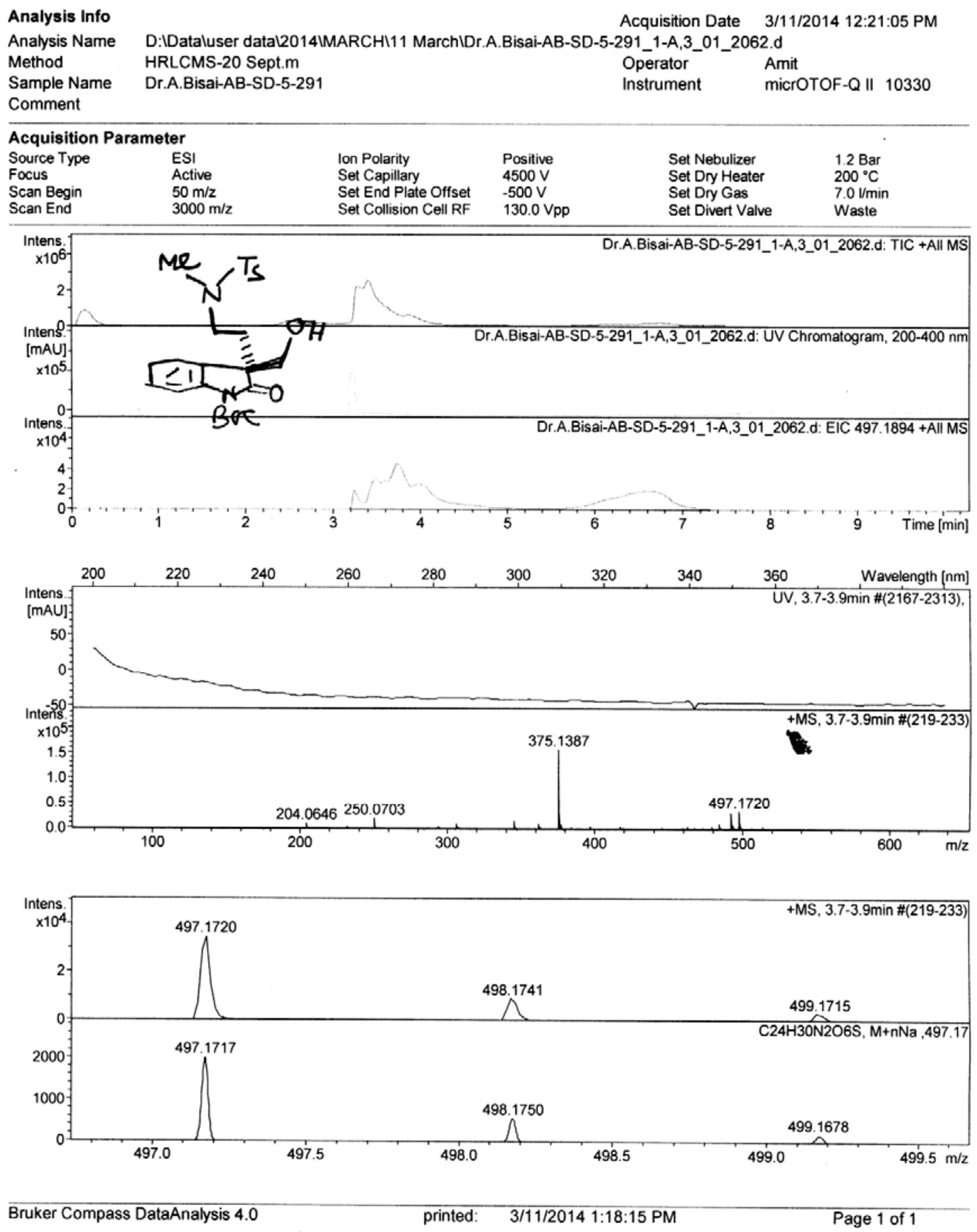

Scanned copy of mass spectrum of (6e) 


\section{HPLC data of ( \pm )-6e:}

Data File C: \CHEM32\1\DATA \SUBHADIP \2014-02-07SD-5-291RACEMIC-ADH-20-1-254-60.D Sample Name: SD-5-291RACEMIC-ADH-20-1-254-60

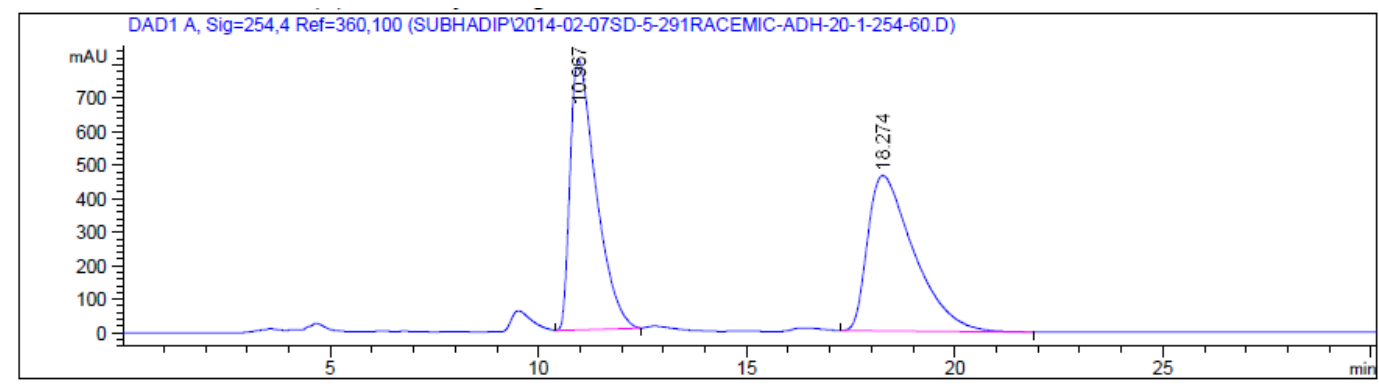

Signal 1: DAD1 A, Sig $=254,4$ Ref $=360,100$

\begin{tabular}{|c|c|c|c|c|c|}
\hline $\begin{array}{c}\text { Peak } \\
\#\end{array}$ & $\begin{array}{l}\text { RetTime Type } \\
\text { [min] }\end{array}$ & $\begin{array}{l}\text { Width } \\
\text { [min] }\end{array}$ & $\begin{array}{c}\text { Area } \\
{\left[\mathrm{mAU}^{*} \mathrm{~s}\right]}\end{array}$ & $\begin{array}{l}\text { Height } \\
{[\mathrm{mAU}]}\end{array}$ & $\begin{array}{c}\text { Area } \\
\%\end{array}$ \\
\hline$-\ldots$ & $-1--$ & - & |........ & |-..... & | \\
\hline 1 & 10.967 BB & 0.6490 & $3.48616 \mathrm{e} 4$ & 805.35229 & 49.3347 \\
\hline 2 & 18. $274 \mathrm{BB}$ & 1.1530 & $3.58019 \mathrm{e} 4$ & 463.57822 & 50.6653 \\
\hline Tota & : & & $7.06636 \mathrm{e} 4$ & 1268.93051 & \\
\hline
\end{tabular}

*** End of Report ***

\section{HPLC data of (+)-6e:}

Data File C: \CHEM32 \1\DATA \SUBHADIP \2014-03-03SD-5-345-ADH-20-1-254-60.D Sample Name: SD-5-345-ADH-20-1-254-60

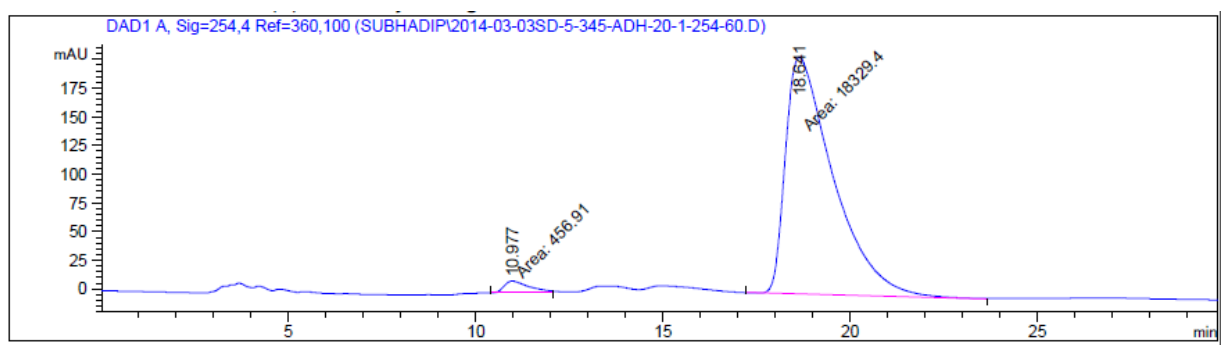

Signal 1: DAD1 A, Sig=254,4 Ref $=360,100$

\begin{tabular}{|c|c|c|c|c|c|}
\hline $\begin{array}{c}\text { Peak } \\
\#\end{array}$ & $\begin{array}{l}\text { RetTime Type } \\
\text { [min] }\end{array}$ & $\begin{array}{l}\text { Width } \\
\text { [min] }\end{array}$ & $\begin{array}{c}\text { Area } \\
{\left[\mathrm{mAU}^{*} \mathrm{~s}\right]}\end{array}$ & $\begin{array}{l}\text { Height } \\
\text { [mAU] }\end{array}$ & $\begin{array}{c}\text { Area } \\
\%\end{array}$ \\
\hline & & & - - & - & | \\
\hline 1 & $10.977 \mathrm{MM}$ & 0.7699 & 456.90955 & 9.89164 & 2.4321 \\
\hline 2 & $18.641 \mathrm{MM}$ & 1.4821 & $1.83294 \mathrm{e} 4$ & 206.11861 & 97.5679 \\
\hline Tota] & ls: & & $1.87863 \mathrm{e} 4$ & 216.01025 & \\
\hline
\end{tabular}




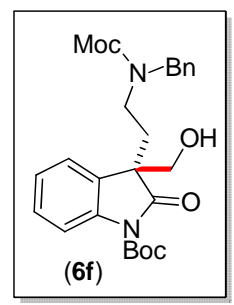

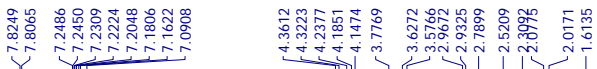

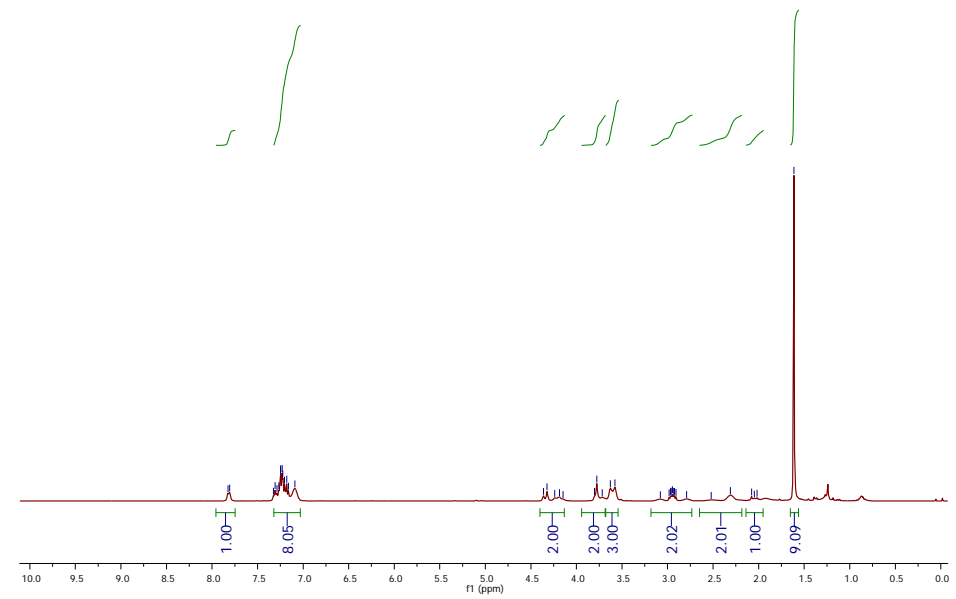

${ }^{1} \mathrm{H}$ NMR (400 MHz, $\mathrm{CDCl}_{3}$ ) of compound (6f)

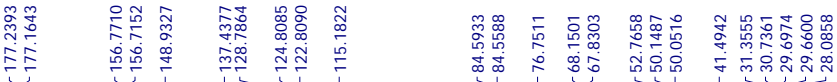

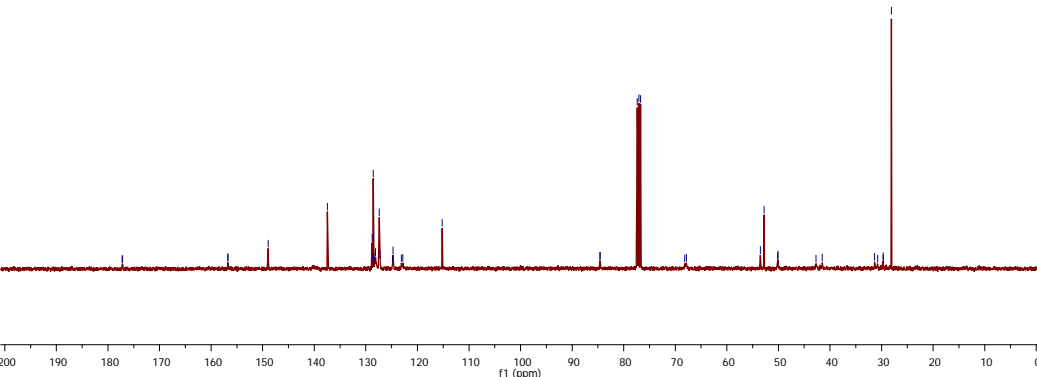

${ }^{13} \mathrm{C}$ NMR $\left(100 \mathrm{MHz}, \mathrm{CDCl}_{3}\right)$ of compound (6f) 


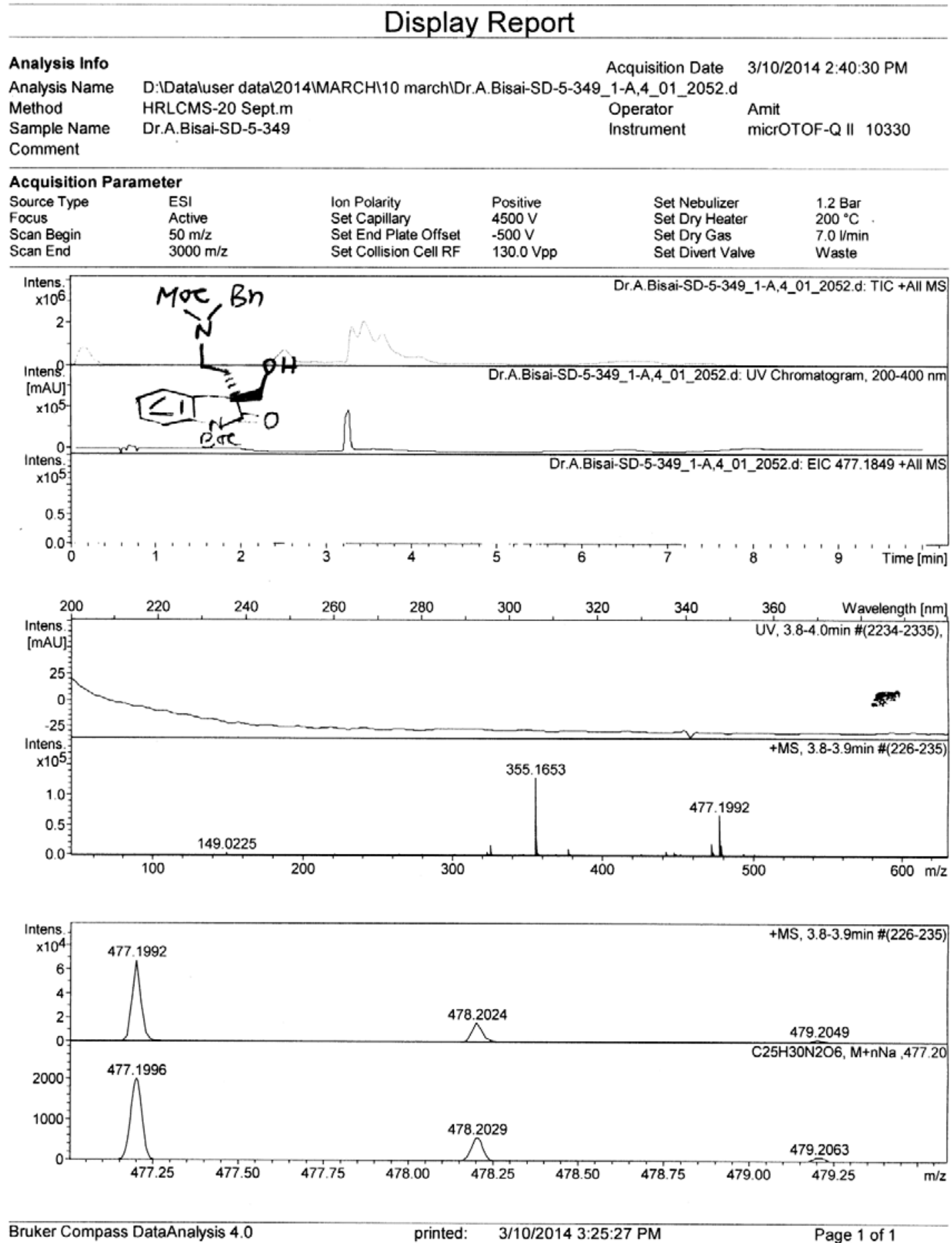

Scanned copy of mass spectrum of ( $6 \mathbf{6})$ 


\section{HPLC data of ( \pm )-6f:}

Data File C: \CHEM32\1\DATA \SUBHADIP \2014-03-05SD-5-349RACEMIC-ADH-10-1-254-60.D Sample Name: SD-5-349Racemic-ADH-10-1-254-60

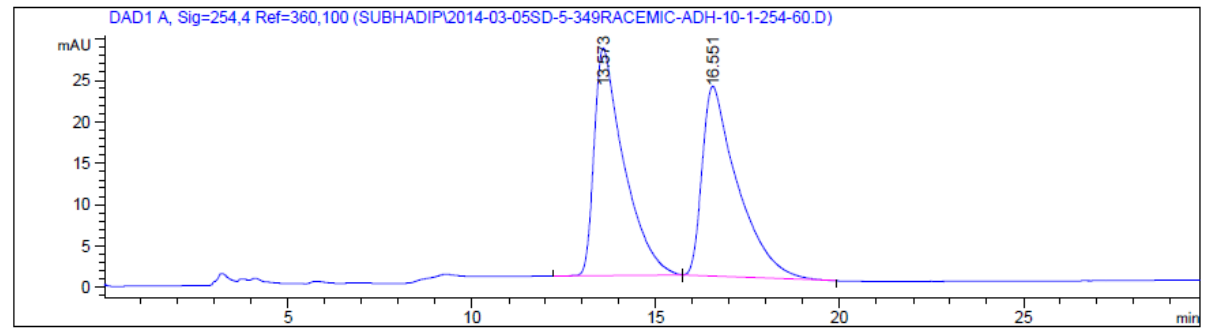

Signal 1: DAD1 A, Sig=254,4 Ref $=360,100$

\begin{tabular}{|c|c|c|c|c|c|}
\hline $\begin{array}{c}\text { Peak } \\
\#\end{array}$ & $\begin{array}{l}\text { RetTime Type } \\
\text { [min] }\end{array}$ & $\begin{array}{l}\text { Width } \\
\text { [min] }\end{array}$ & $\begin{array}{c}\text { Area } \\
{\left[\mathrm{mAU}^{*} \mathrm{~s}\right]}\end{array}$ & $\begin{array}{l}\text { Height } \\
\text { [mAU] }\end{array}$ & $\begin{array}{c}\text { Area } \\
\%\end{array}$ \\
\hline$\cdots$ & |-..-- $\mid--$ & $\ldots$ & 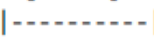 & - & 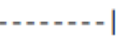 \\
\hline 1 & $13.573 \mathrm{BB}$ & 0.7958 & 1544.30542 & 27.47456 & 50.0964 \\
\hline 2 & $16.551 \mathrm{BB}$ & 0.9423 & 1538.36035 & 22.94315 & 49.9036 \\
\hline \multicolumn{3}{|c|}{ Totals : } & 3082.66577 & 50.41771 & \\
\hline
\end{tabular}

*** End of Report ***

\section{HPLC data of (+)-6f:}

Data File C: \CHEM32\1\DATA \SUBHADIP \2014-03-07SD-5--351-ADH-10-1-254-60.D Sample Name: SD-5--351-ADH-10-1-254-60

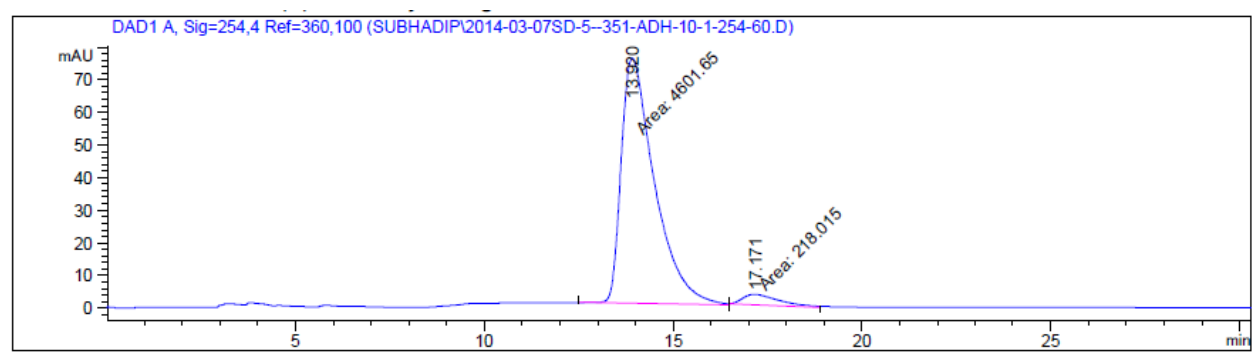

Signal 1: DAD1 A, Sig=254,4 Ref $=360,100$

\begin{tabular}{|c|c|c|c|c|c|}
\hline $\begin{array}{c}\text { Peak } \\
\quad \#\end{array}$ & $\begin{array}{l}\text { RetTime Type } \\
\text { [min] }\end{array}$ & $\begin{array}{l}\text { Width } \\
\text { [min] }\end{array}$ & $\begin{array}{c}\text { Area } \\
{\left[\mathrm{mAU}^{*} \mathrm{~s}\right]}\end{array}$ & $\begin{array}{l}\text { Height } \\
\text { [mAU] }\end{array}$ & $\begin{array}{c}\text { Area } \\
\%\end{array}$ \\
\hline & & & $1=-1$ & & 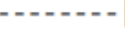 \\
\hline 1 & $13.920 \mathrm{MM}$ & 1.0209 & 4601.65186 & 75.12724 & 95.4765 \\
\hline 2 & $17.171 \mathrm{MM}$ & 1.1191 & 218.01534 & 3.24695 & 4.5235 \\
\hline Tota & : & & 4819.66719 & 78.37418 & \\
\hline
\end{tabular}

*** End of Report *** 


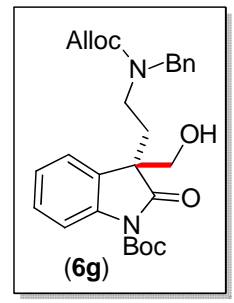

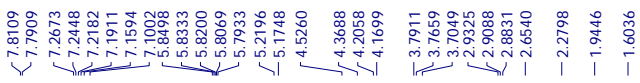

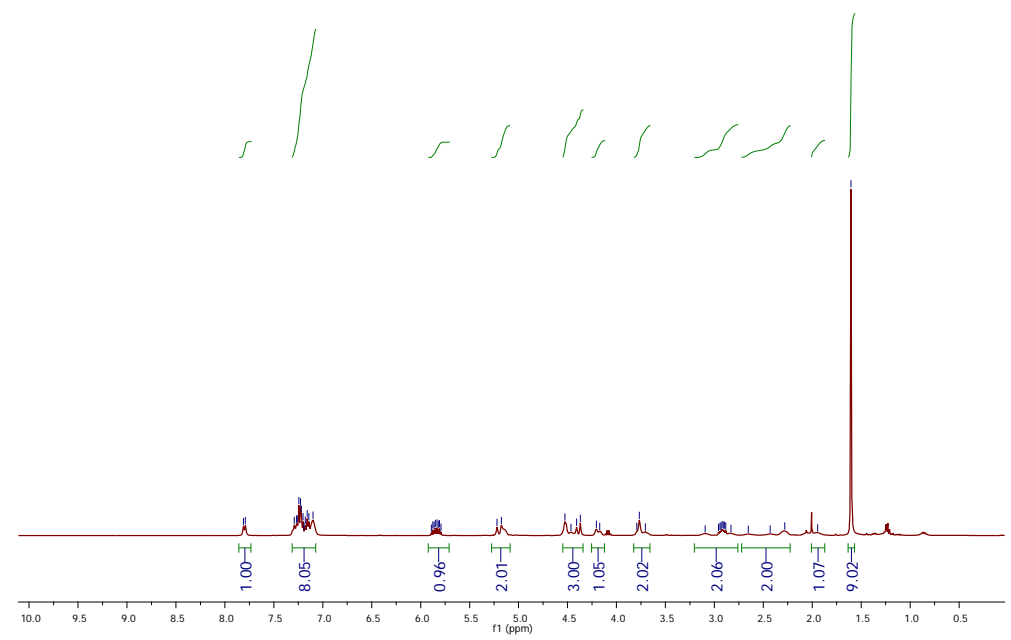

${ }^{1} \mathrm{H}$ NMR (400 MHz, $\mathrm{CDCl}_{3}$ ) of compound (6g)

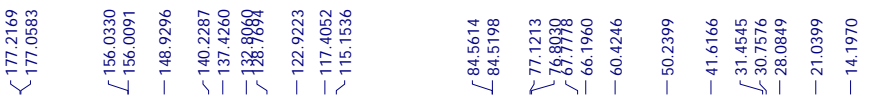
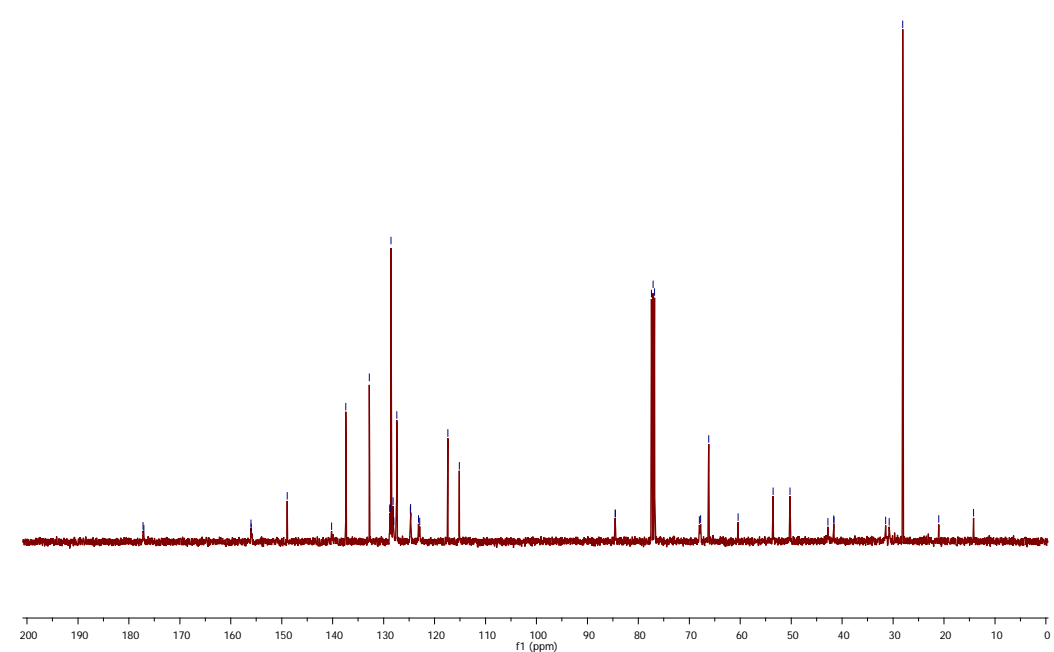

${ }^{13} \mathrm{C}$ NMR (100 MHz, $\mathrm{CDCl}_{3}$ ) of compound (6g) 


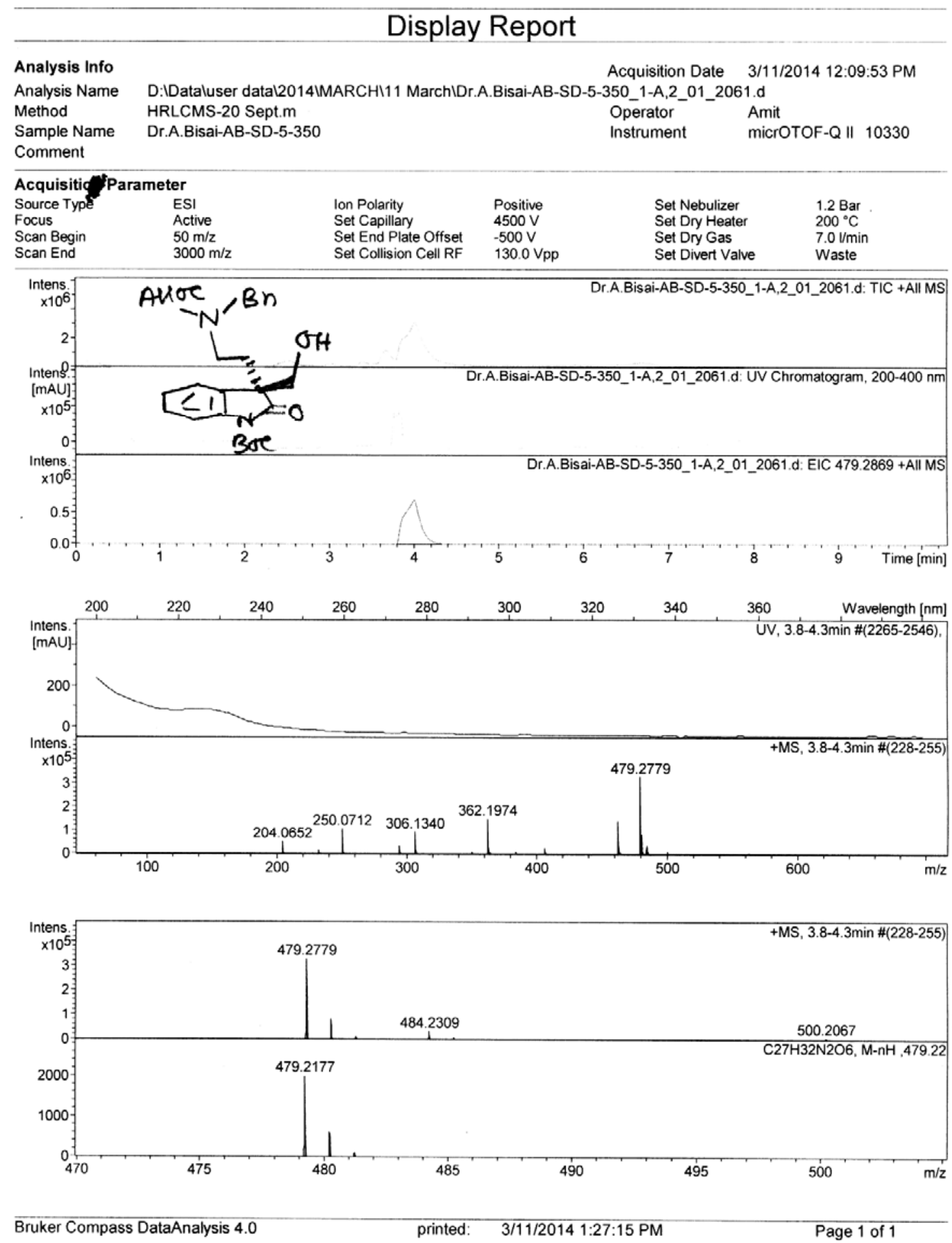

Scanned copy of mass spectrum of $(\mathbf{6 g})$ 


\section{HPLC data of ( \pm )-6g:}

Data File C: \CHEM32\1\DATA \SUBHADIP $\backslash 2014-03-05 S D-5-350$ RACEMIC-ADH-10-1-254-60.D Sample Name: SD-5-350Racemic-ADH-10-1-254-60

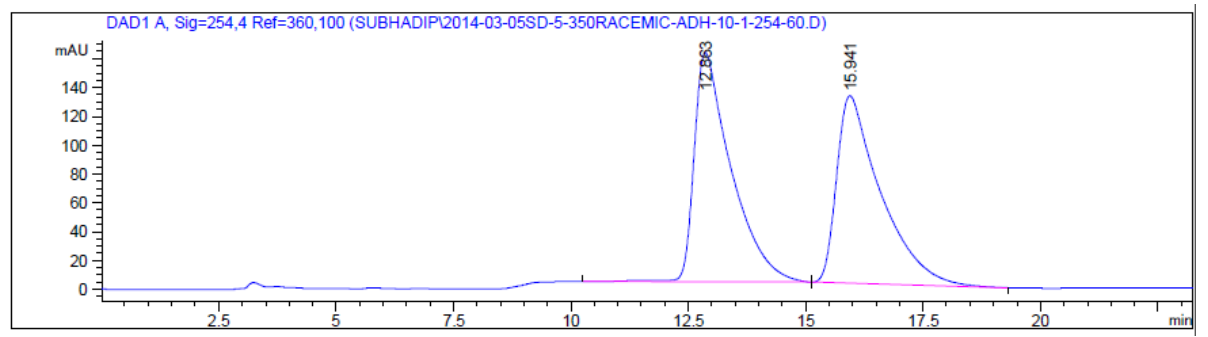

Signal 1: DAD1 A, Sig=254,4 Ref $=360,100$

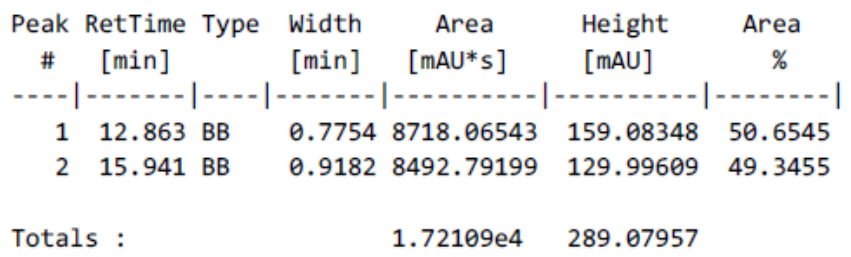

*** End of Report ***

\section{HPLC data of (-)-6g:}

Data File C: \CHEM32\1\DATA \SUBHADIP \2014-03-07SD-5--352-ADH-10-1-254-60.D Sample Name: SD-5--352-ADH-10-1-254-60

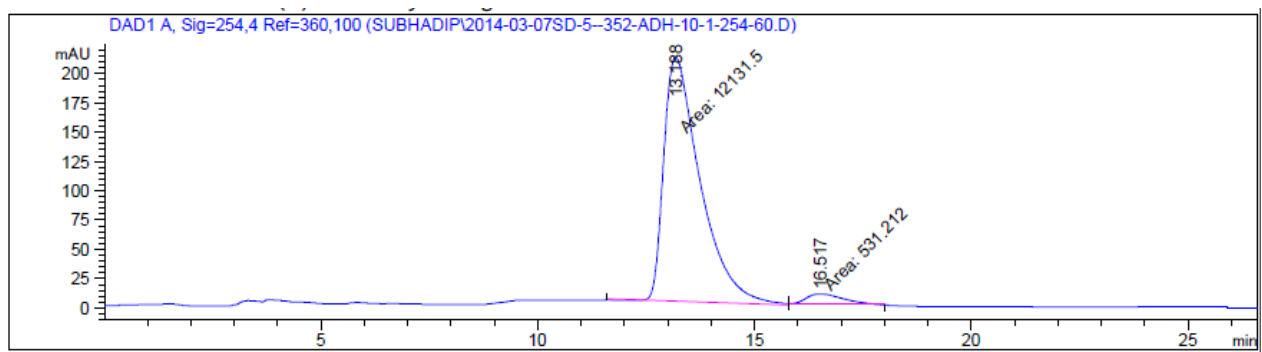

Signal 1: DAD1 A, Sig $=254,4$ Ref $=360,100$

\begin{tabular}{cccccc}
$\begin{array}{c}\text { Peak RetTime Type } \\
\text { \# } \\
\text { [min] }\end{array}$ & $\begin{array}{c}\text { Width } \\
\text { [min] }\end{array}$ & $\begin{array}{c}\text { Area } \\
\text { [mAU*s] }\end{array}$ & \multicolumn{1}{c}{$\begin{array}{c}\text { Height } \\
\text { [mAU] }\end{array}$} & $\begin{array}{c}\text { Area } \\
\%\end{array}$ \\
\hline 1 & 13.188 MM & 0.9726 & $1.21315 \mathrm{e} 4$ & 207.88571 & 95.8049 \\
2 & 16.517 MM & 0.9741 & 531.21228 & 9.08897 & 4.1951 \\
& & & & \\
Totals : & & $1.26628 \mathrm{e} 4$ & 216.97468 &
\end{tabular}

*** End of Report *** 


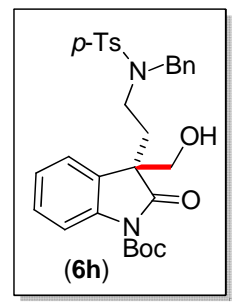

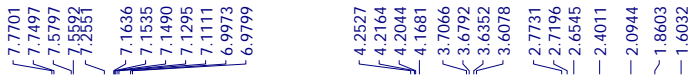
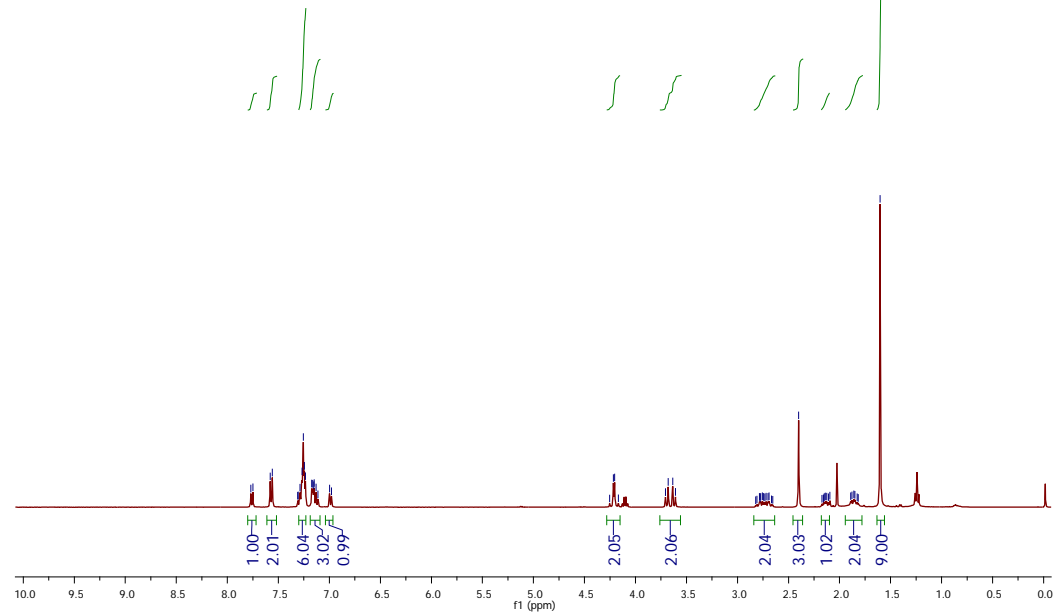

${ }^{1} \mathrm{H}$ NMR (400 MHz, $\mathrm{CDCl}_{3}$ ) of compound (6h)

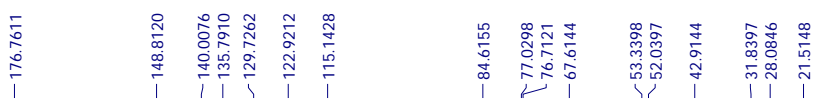
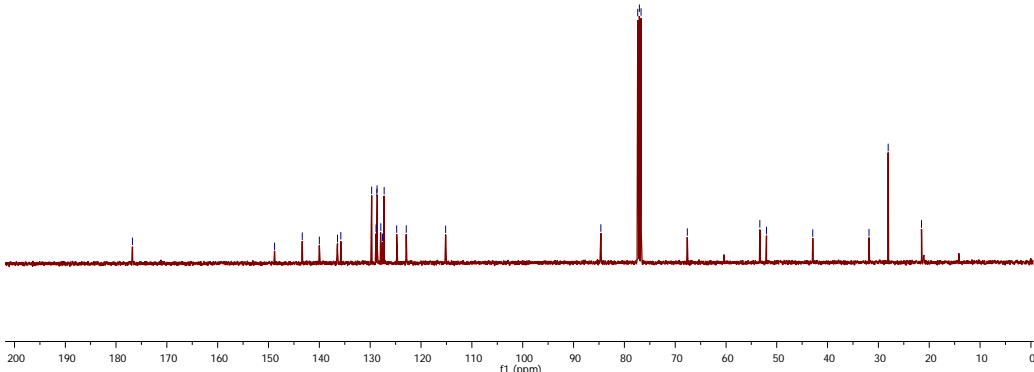

${ }^{13} \mathrm{C}$ NMR $\left(100 \mathrm{MHz}, \mathrm{CDCl}_{3}\right)$ of compound (6h) 


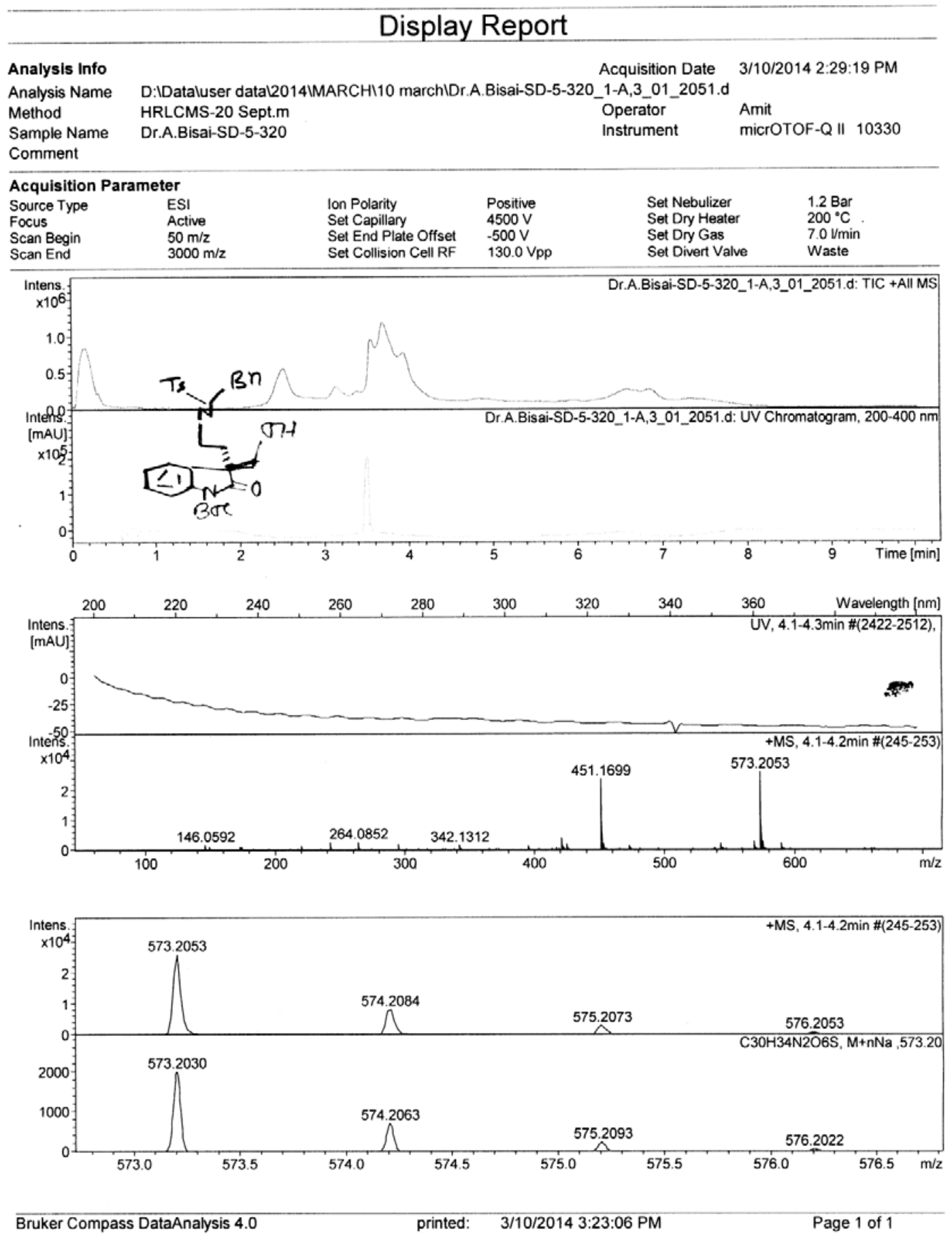

Scanned copy of mass spectrum of (6h) 


\section{HPLC data of $( \pm)-6 h:$}

Data File C: \CHEM32\1\DATA \SUBHADIP \2014-02-19SD-5-320RAC-ADH-20-254-1-60.D Sample Name: SD-5-320Rac-ADH-20-254-1-60

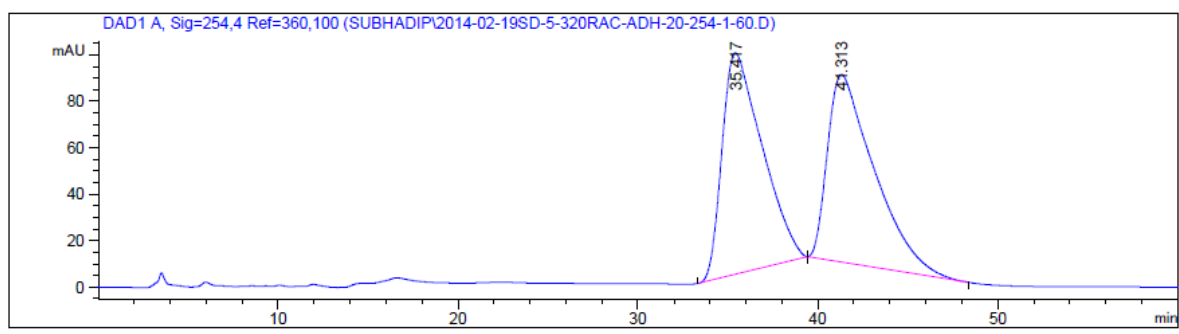

Signal 1: DAD1 A, Sig $=254,4$ Ref $=360,100$

\begin{tabular}{|c|c|c|c|c|c|}
\hline $\begin{array}{c}\text { Peak } \\
\#\end{array}$ & $\begin{array}{l}\text { RetTime Type } \\
\text { [min] }\end{array}$ & $\begin{array}{l}\text { Width } \\
\text { [min] }\end{array}$ & $\begin{array}{c}\text { Area } \\
{\left[\mathrm{mAU}{ }^{*} \mathrm{~s}\right]}\end{array}$ & $\begin{array}{l}\text { Height } \\
{[\mathrm{mAU}]}\end{array}$ & $\begin{array}{c}\text { Area } \\
\%\end{array}$ \\
\hline-- & $1-$ & 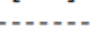 & |-- & - & | \\
\hline 1 & $35.417 \mathrm{BB}$ & 2.2190 & $1.47435 \mathrm{e} 4$ & 95.39864 & 50.1422 \\
\hline 2 & $41.313 \mathrm{BB}$ & 2.5788 & $1.46599 \mathrm{e} 4$ & 80.87445 & 49.8578 \\
\hline Tota & Is: & & $2.94033 \mathrm{e} 4$ & 176.27309 & \\
\hline
\end{tabular}

*** End of Report ***

\section{HPLC data of (-)-6h:}

Data File C: \CHEM32\1\DATA \SUBHADIP \2014-03-03SD-5-346-ADH-20-1-254-60.D Sample Name: SD-5-346-ADH-20-1-254-60

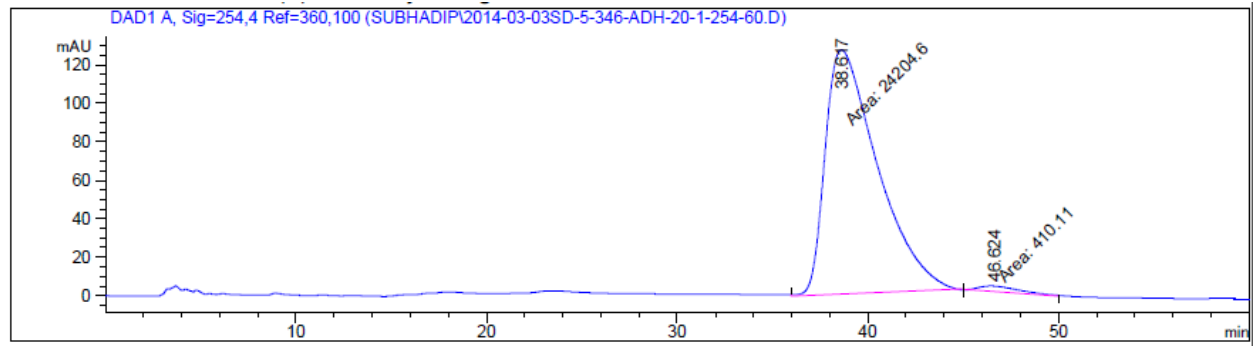

Signal 1: DAD1 A, Sig=254,4 Ref=360,100

\begin{tabular}{|c|c|c|c|c|c|c|}
\hline $\begin{array}{c}\text { Peak } \\
\#\end{array}$ & $\begin{array}{c}\text { RetTime } \\
\text { [min] }\end{array}$ & Type & $\begin{array}{l}\text { Width } \\
\text { [min] }\end{array}$ & $\begin{array}{c}\text { Area } \\
{\left[\mathrm{mAU}^{*} \mathrm{~s}\right]}\end{array}$ & $\begin{array}{l}\text { Height } \\
\text { [mAU] }\end{array}$ & $\begin{array}{c}\text { Area } \\
\%\end{array}$ \\
\hline-1 & |-- & & ---1 & 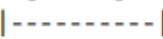 & - - - & - \\
\hline 1 & 38.617 & MM & 3.1835 & $2.42046 \mathrm{e} 4$ & 126.71757 & 98.3339 \\
\hline 2 & 46.624 & MM & 2.4267 & 410.11044 & 2.81671 & 1.6661 \\
\hline$d \perp$ & 15. & & & $2.46147 e 4$ & 129.53428 & \\
\hline
\end{tabular}

*** End of Report *** 


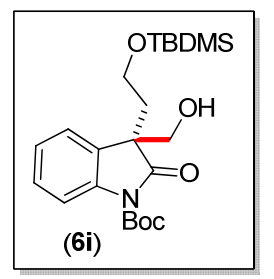

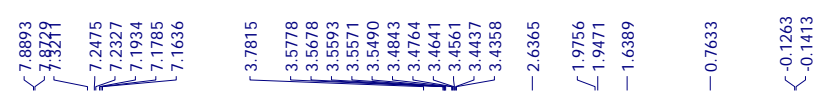

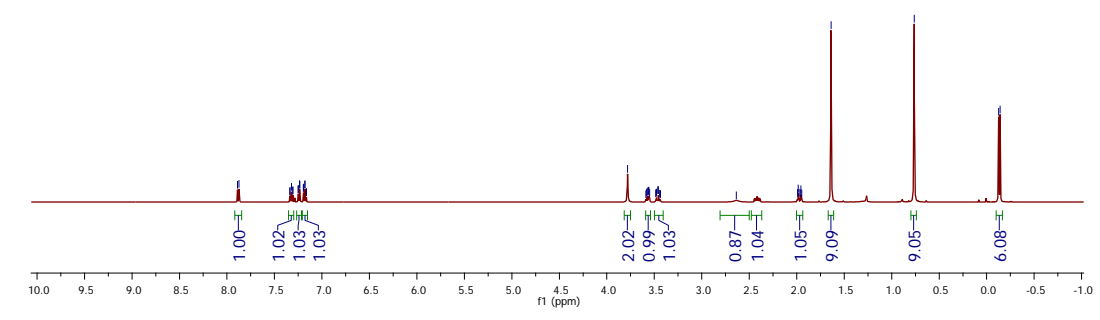

${ }^{1} \mathrm{H}$ NMR (1900 MHz, $\mathrm{CDCl}_{3}$ ) of compound (6i)

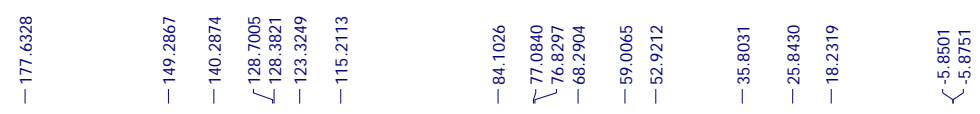

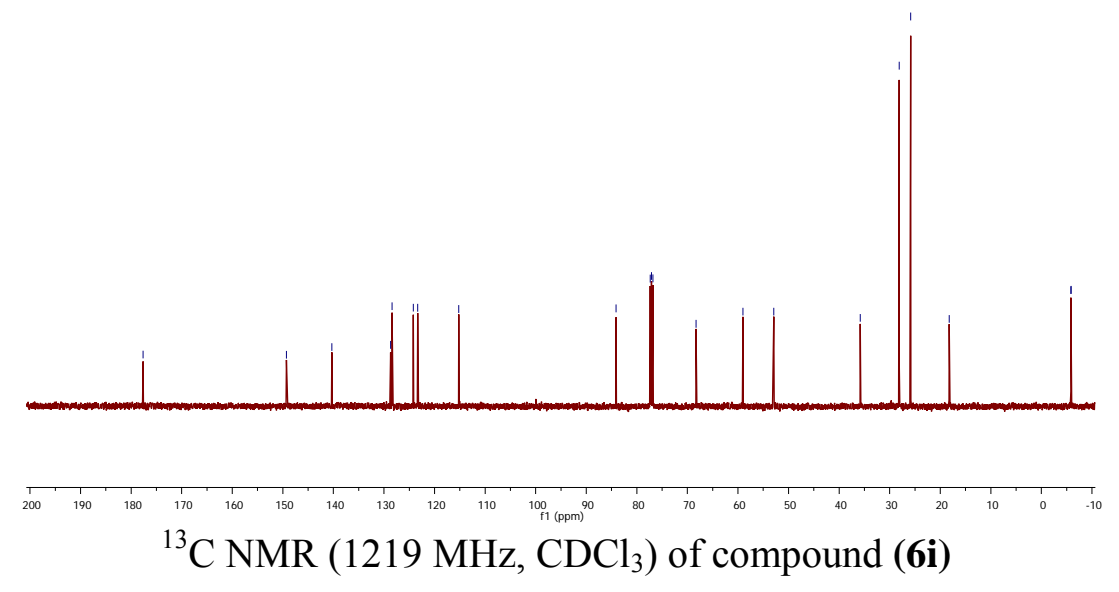



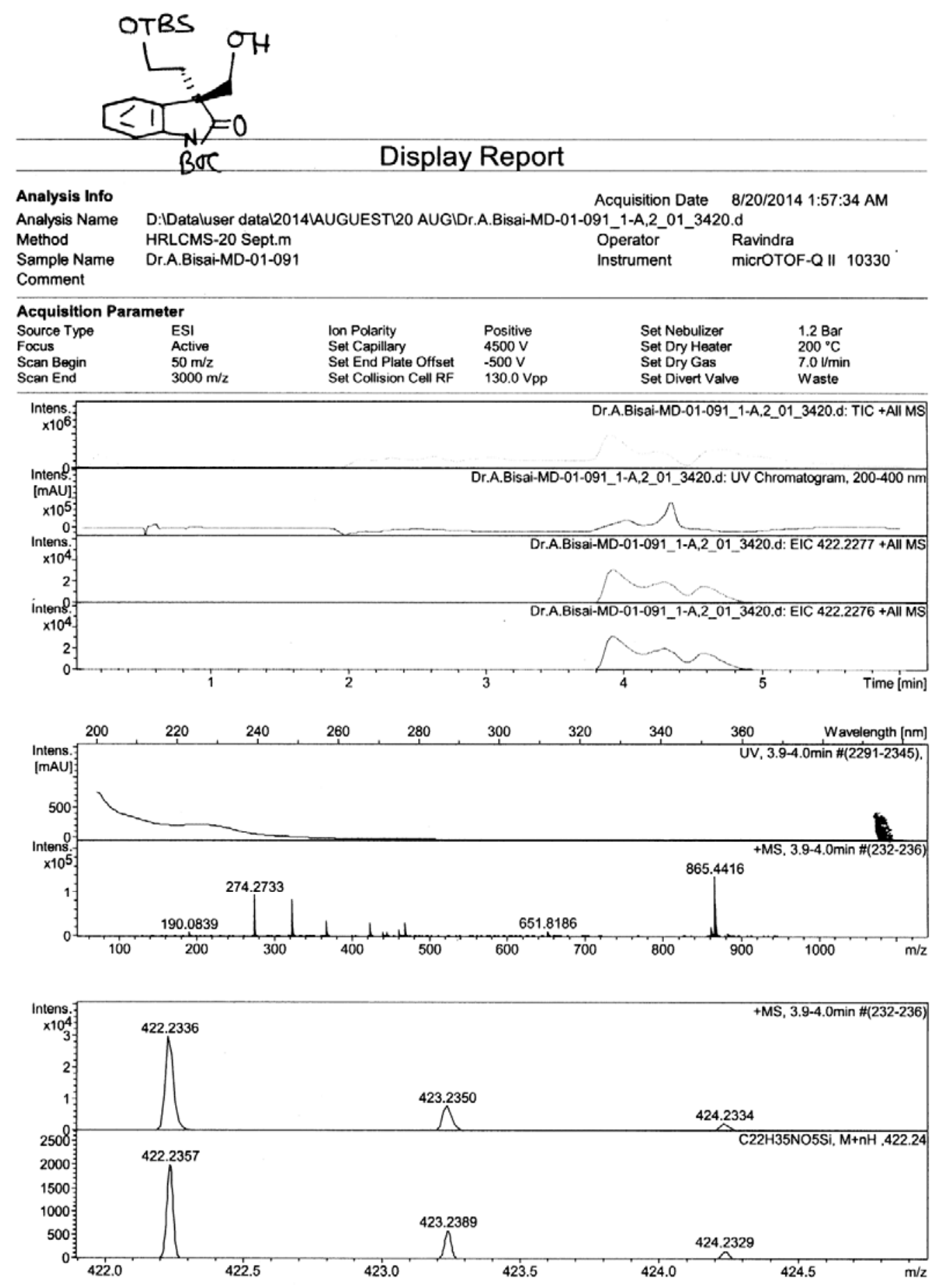

Bruker Compass DataAnalysis 4.0 $\quad$ printed: $\quad 8 / 20 / 2014$ 3:43:45 AM $\quad$ Page 1 of 1

Scanned copy of mass spectrum of $(\mathbf{6 i})$ 


\section{HPLC data of ( \pm )-6i:}

Data File C: \CHEM32\1\DATA \SUBHADIP \2014-03-14MD-1-91RACEMIC-IC-5-1-254-60.D Sample Name: MD-1-91RACEMIC-IC-5-1-254-60

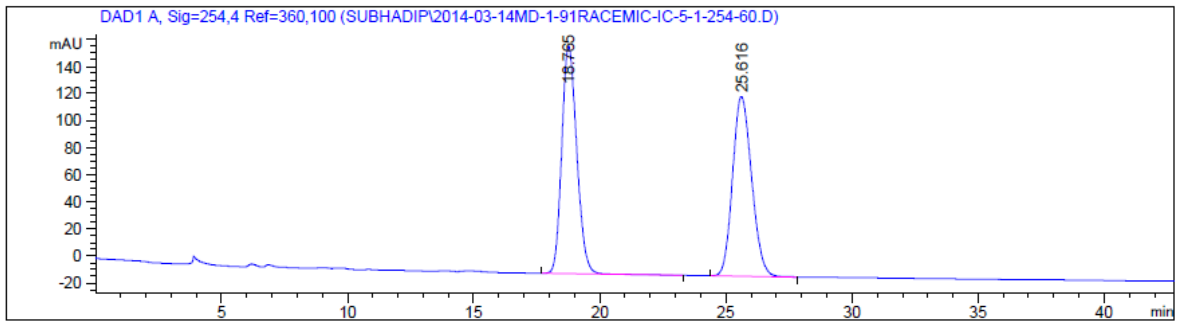

Signal 1: DAD1 A, Sig=254,4 Ref $=360,100$

\begin{tabular}{cccccc}
$\begin{array}{c}\text { Peak RetTime Type } \\
\text { Width } \\
\text { [min] }\end{array}$ & $\begin{array}{c}\text { Area } \\
{[\mathrm{min}]}\end{array}$ & $\begin{array}{c}\text { Height } \\
{[\mathrm{mAU} \text { s }]}\end{array}$ & $\begin{array}{c}\text { Area } \\
{[\mathrm{mAU}]}\end{array}$ & $\%$ \\
\hline 1 & 18.765 BB & 0.6425 & 6946.16748 & 167.96336 & 49.9113 \\
2 & 25.616 BBA & 0.8210 & 6970.85449 & 132.66325 & 50.0887 \\
& & & & \\
Totals : & & $1.39170 \mathrm{e} 4$ & 300.62662 &
\end{tabular}

*** End of Report ***

\section{HPLC data of (-)-6i:}

Data File C: \CHEM32\1\DATA \SUBHADIP \2014-03-21SD-5-375-IC-5-254-1-60.D Sample Name: SD-5-375-IC-5-254-1-60

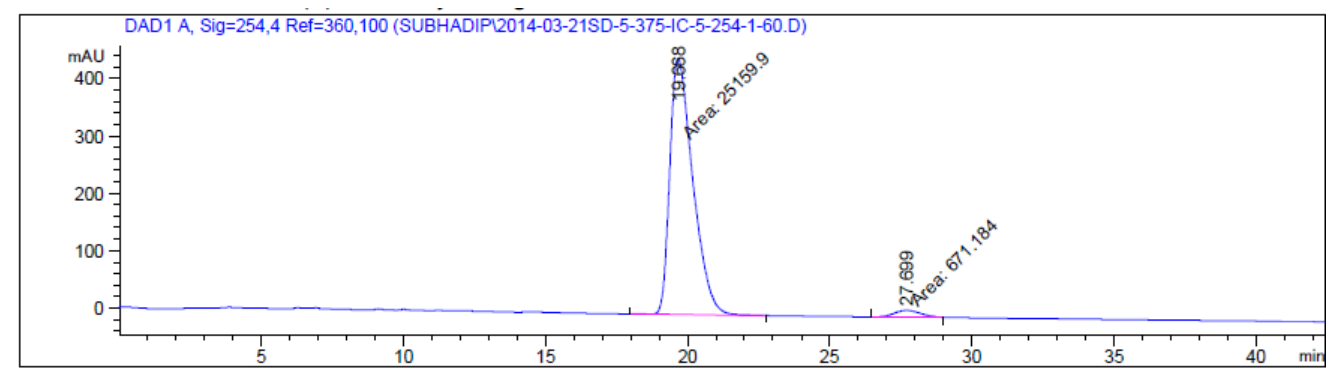

Signal 1: DAD1 A, Sig $=254,4$ Ref $=360,100$

\begin{tabular}{|c|c|c|c|c|c|c|}
\hline $\begin{array}{c}\text { Peak } \\
\#\end{array}$ & $\begin{array}{c}\text { RetTime } \\
\text { [min] }\end{array}$ & Type & $\begin{array}{l}\text { Width } \\
\text { [min] }\end{array}$ & $\begin{array}{c}\text { Area } \\
{\left[\mathrm{mAU}^{*} \mathrm{~s}\right]}\end{array}$ & $\begin{array}{l}\text { Height } \\
\text { [mAU] }\end{array}$ & $\begin{array}{c}\text { Area } \\
\%\end{array}$ \\
\hline 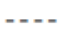 & & & & 1-1 & $\ldots$ & $\ldots$ \\
\hline 1 & 19.668 & MM & 0.9427 & $2.51599 \mathrm{e} 4$ & 444.83441 & 97.4016 \\
\hline 2 & 27.699 & MM & 1.0067 & 671.18378 & 11.11246 & 2.5984 \\
\hline ota & : & & & $2.58310 \mathrm{e} 4$ & 455.94687 & \\
\hline
\end{tabular}

*** End of Report *** 


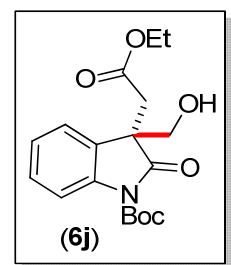

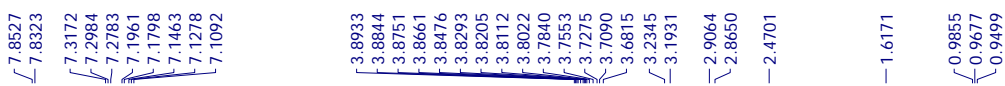

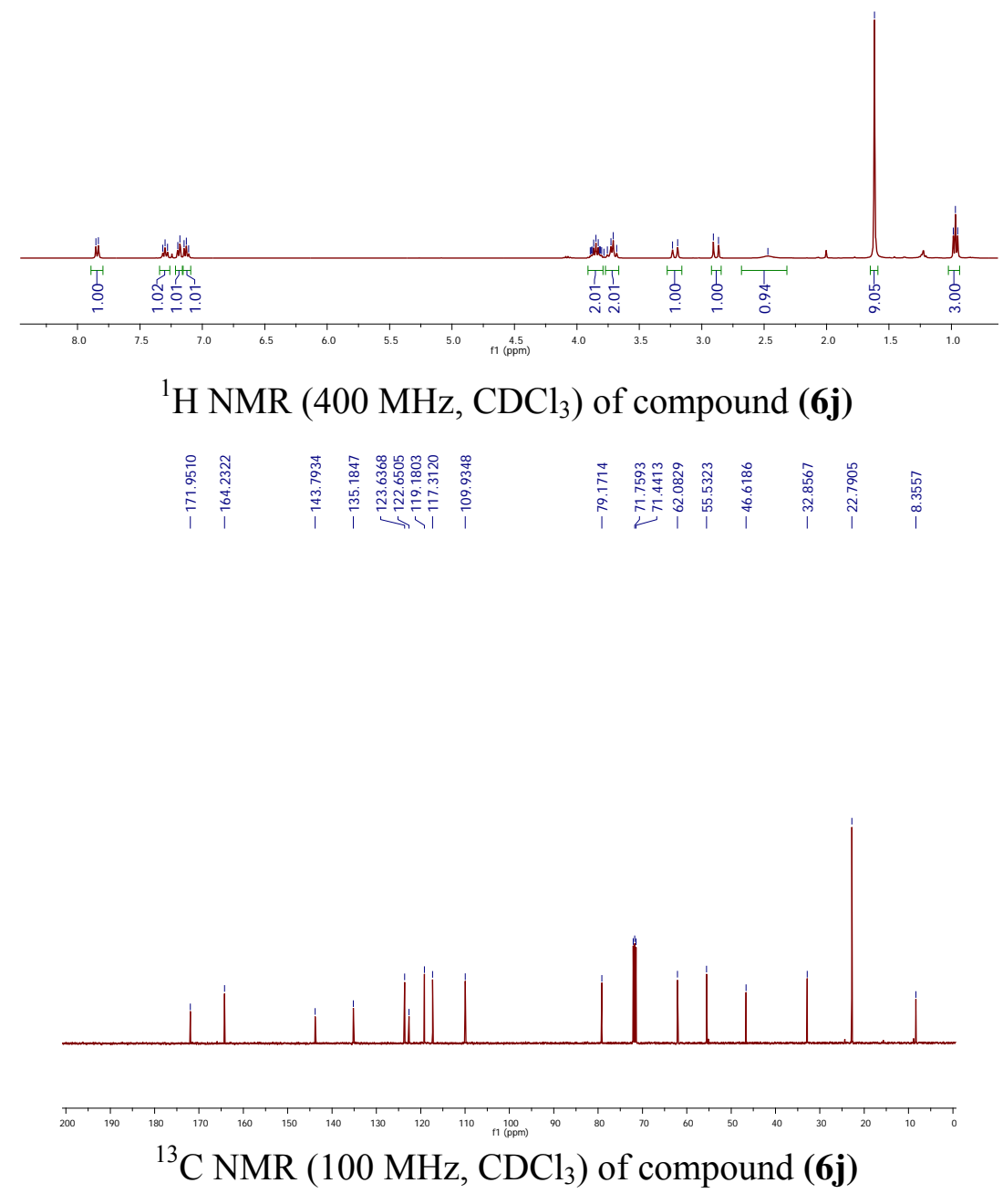



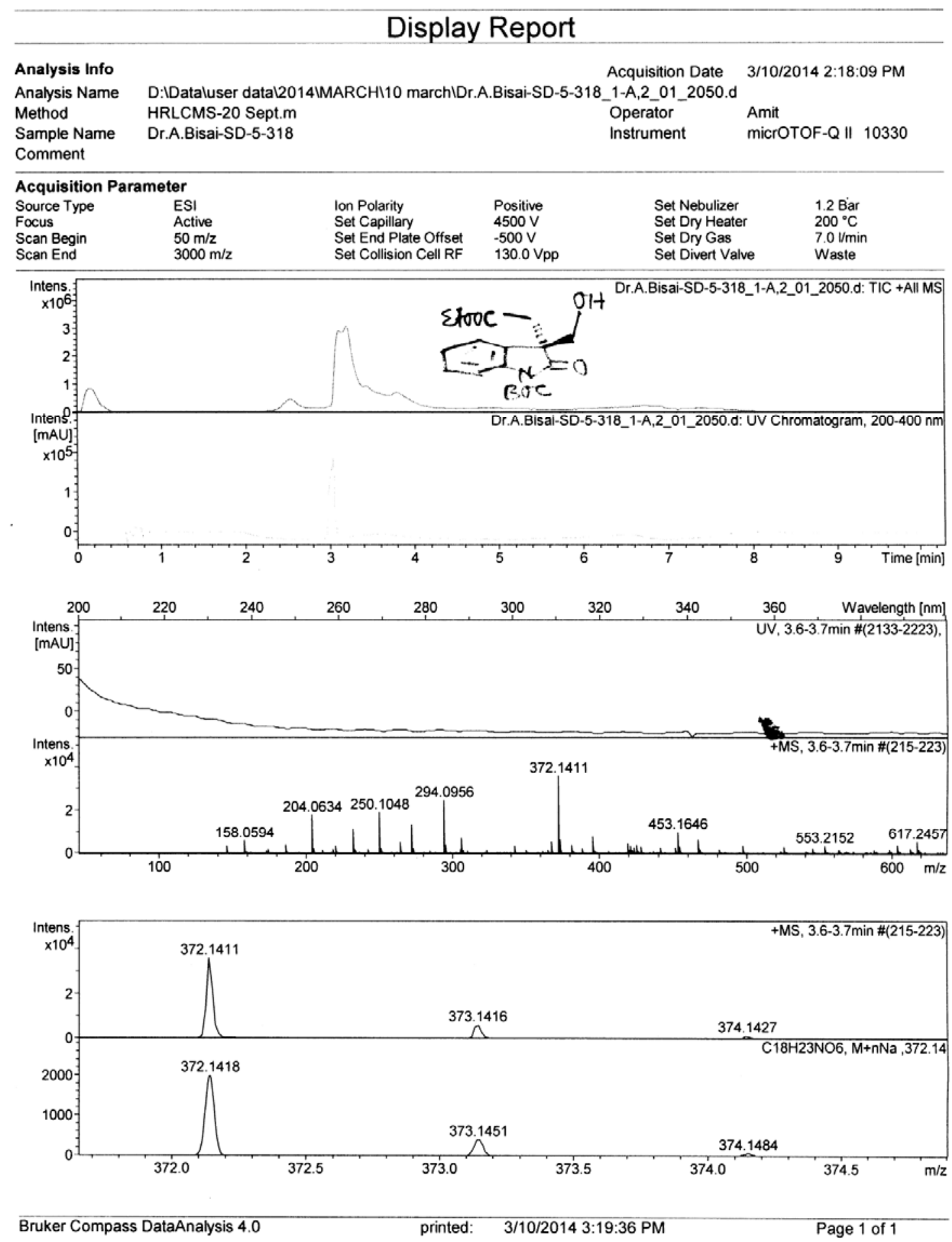

Scanned copy of mass spectrum of $(\mathbf{6} \mathbf{j})$ 


\section{HPLC data of ( \pm$)-6 \mathbf{j}:$}

Data File C: \CHEM32\1\DATA\SUBHADIP\2014-02-17SD-5-305RAC-IC3-30-254-1-60.D Sample Name: SD-5-305Rac-IC3-30-254-1-60

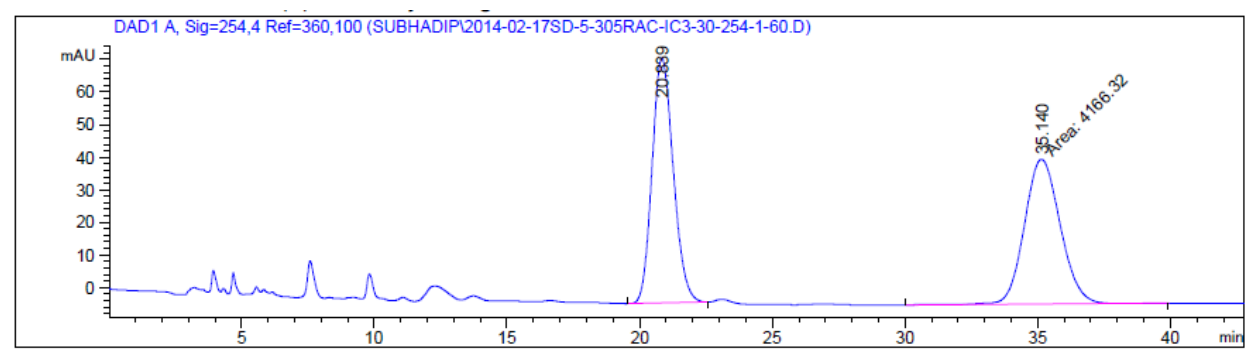

Signal 1: DAD1 A, Sig=254,4 Ref $=360,100$

\begin{tabular}{cccccc}
$\begin{array}{c}\text { Peak RetTime Type } \\
\text { Width } \\
\text { [min] }\end{array}$ & $\begin{array}{c}\text { Area } \\
\text { [min] }\end{array}$ & $\begin{array}{c}\text { Height } \\
\text { [mAU*s] }\end{array}$ & $\begin{array}{c}\text { Area } \\
\text { [mAU] }\end{array}$ & $\%$ \\
\hline 1 & 20.839 BB & 0.8642 & 4181.80566 & 75.26684 & 50.0927 \\
2 & 35.140 MM & 1.5609 & 4166.32129 & 44.48652 & 49.9073 \\
& & & & & \\
Totals : & & 8348.12695 & 119.75335
\end{tabular}

*** End of Report ***

\section{HPLC data of (+)-6j:}

Data File C: \CHEM32\1\DATA \SUBHADIP \2014-02-17SD-5-314-IC3-30-254-1-60.D Sample Name: SD-5-316-IC3-30-254-1-60

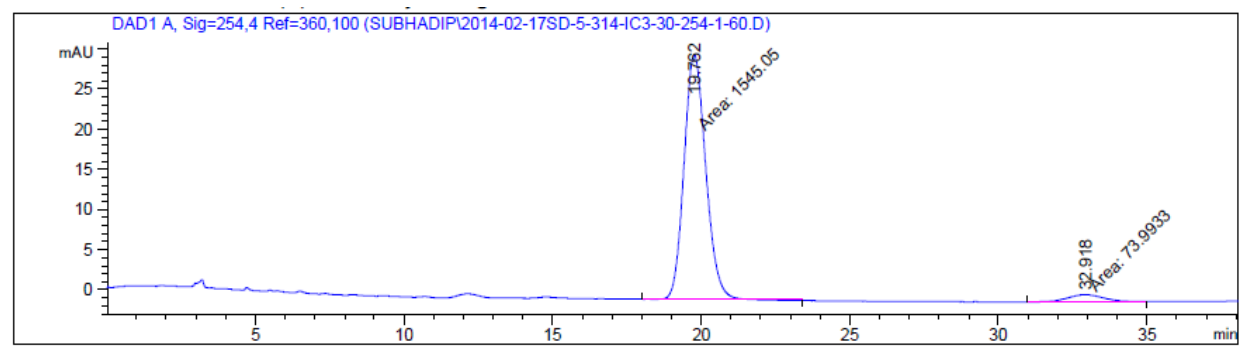

Signal 1: DAD1 A, Sig=254,4 Ref $=360,100$

\begin{tabular}{cccccc}
$\begin{array}{c}\text { Peak RetTime Type } \\
\text { \# }\end{array}$ & $\begin{array}{c}\text { Width } \\
{[\text { min] }}\end{array}$ & $\begin{array}{c}\text { Area } \\
{[\text { min] }}\end{array}$ & $\begin{array}{c}\text { Height } \\
{[\text { mAU*s }]}\end{array}$ & $\begin{array}{c}\text { Area } \\
{[\mathrm{mAU}]}\end{array}$ & $\%$ \\
\hline 1 & 19.762 MM & 0.8457 & 1545.04529 & 30.44781 & 95.4298 \\
2 & 32.918 MM & 1.3957 & 73.99329 & $8.83612 \mathrm{e}-1$ & 4.5702
\end{tabular}

Totals :

$1619.03858 \quad 31.33142$ 

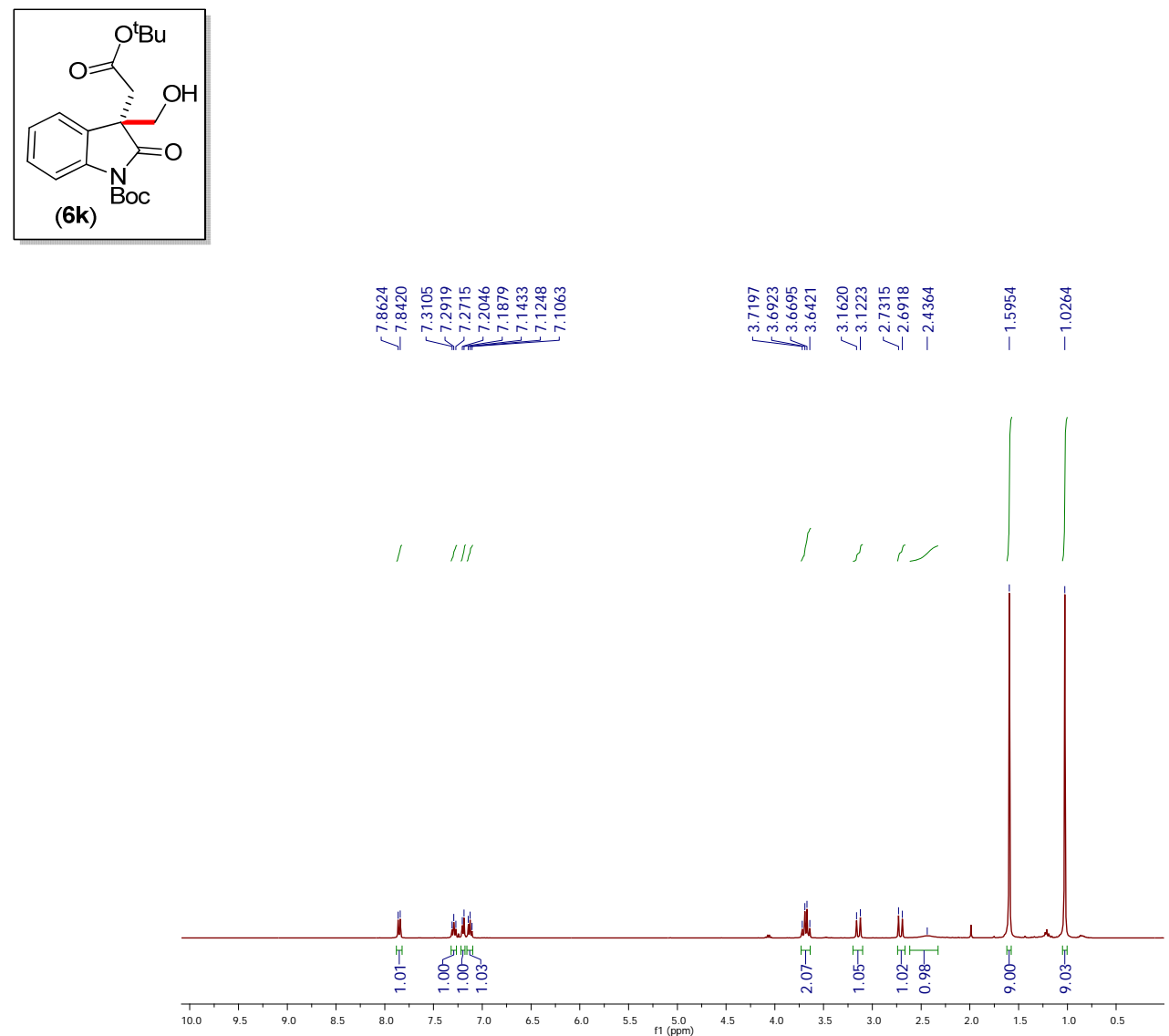

${ }^{1} \mathrm{H}$ NMR (400 MHz, $\mathrm{CDCl}_{3}$ ) of compound (6k)

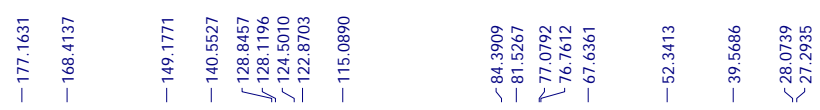

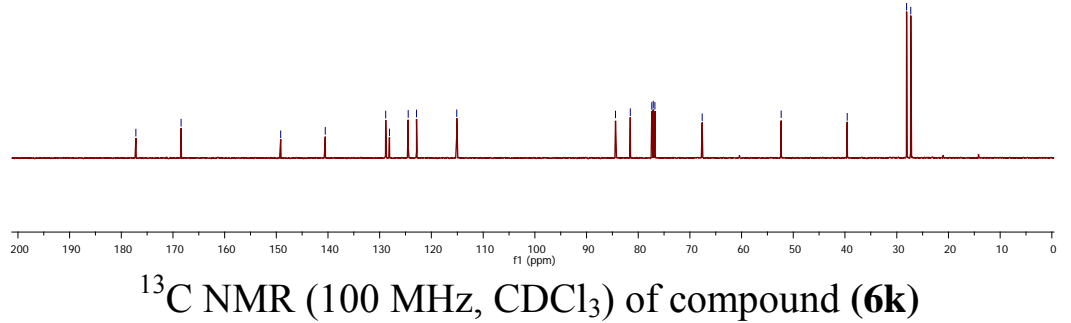




\section{Display Report}
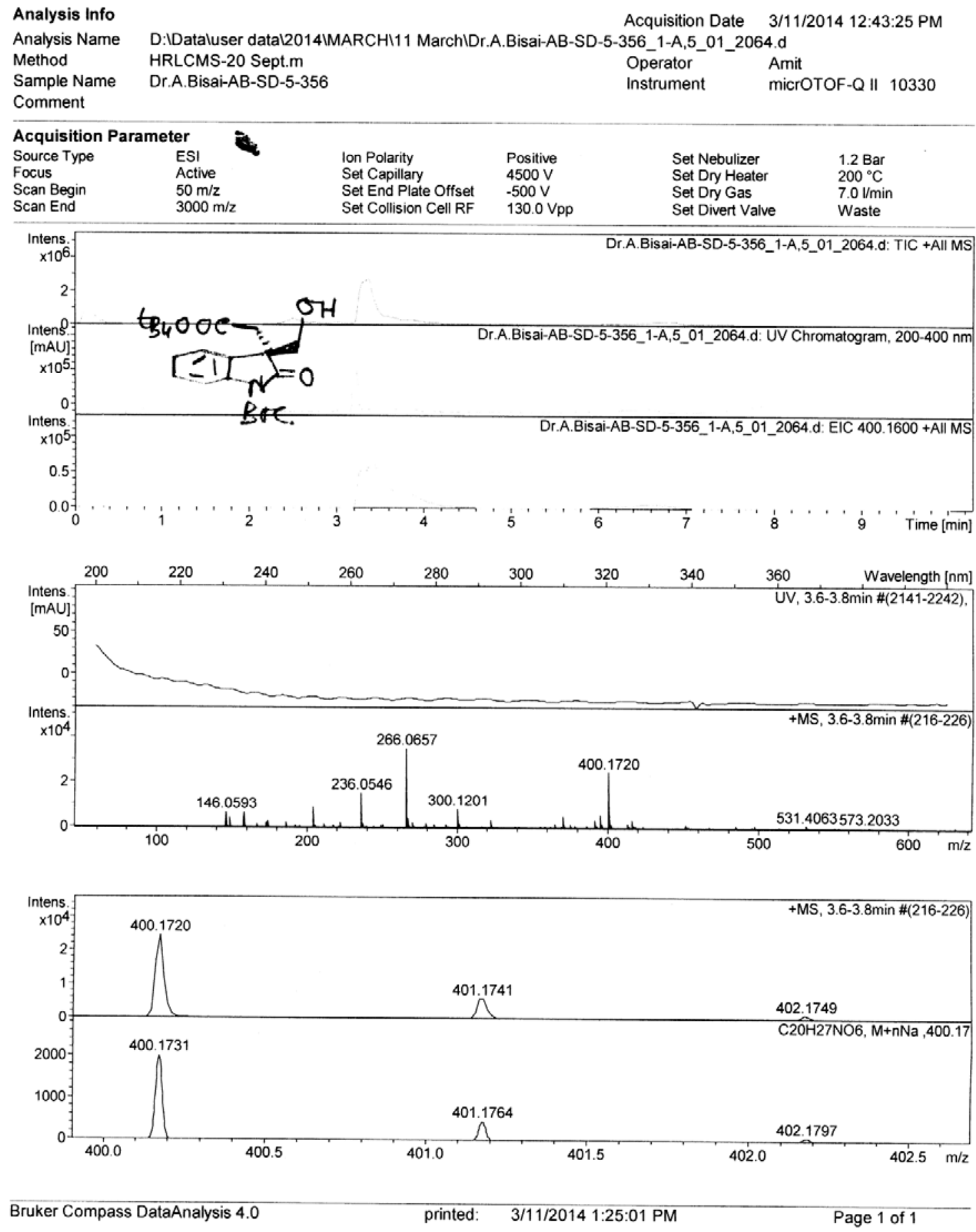

Scanned copy of mass spectrum of (6k) 


\section{HPLC data of ( \pm )-6k:}

Data File C: \CHEM32\1\DATA\SUBHADIP \2014-03-07SD-5-356RACEMIC-IC-30-2-254-30.D Sample Name: SD-5-356RACEMIC-IC-30-2-254-30

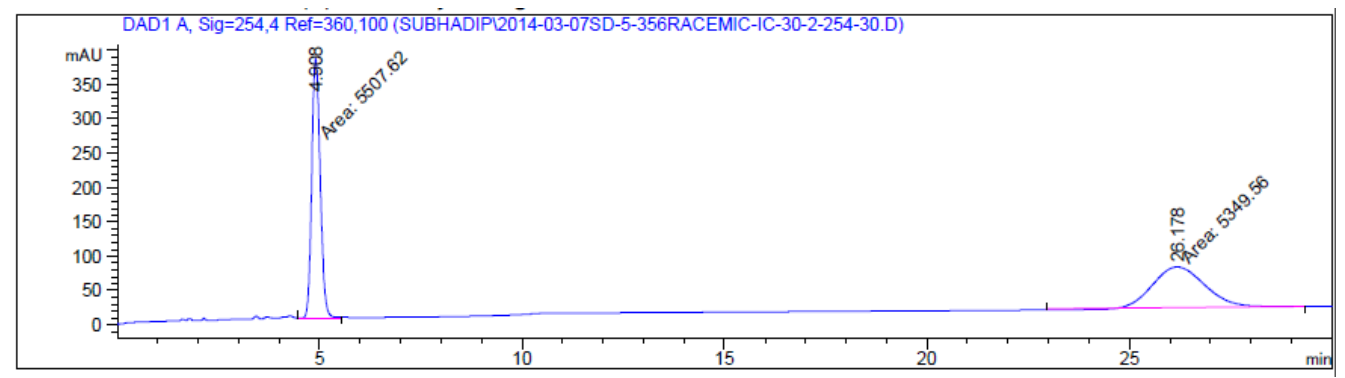

Signal 1: DAD1 A, Sig=254,4 Ref=360,100

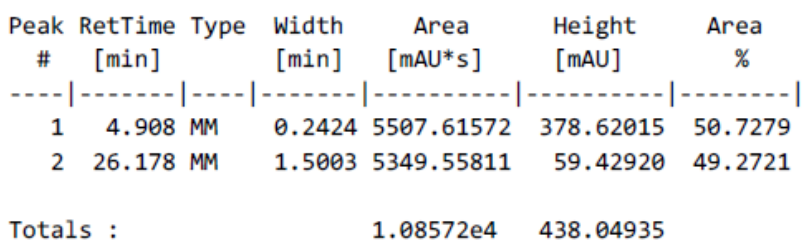

*** End of Report ***

\section{HPLC data of (-)-6k:}

Data File C: \CHEM32\1\DATA \SUBHADIP \2014-03-08SD-5-358-IC-30-2-254-40.D Sample Name: SD-5-358-IC-30-2-254-40

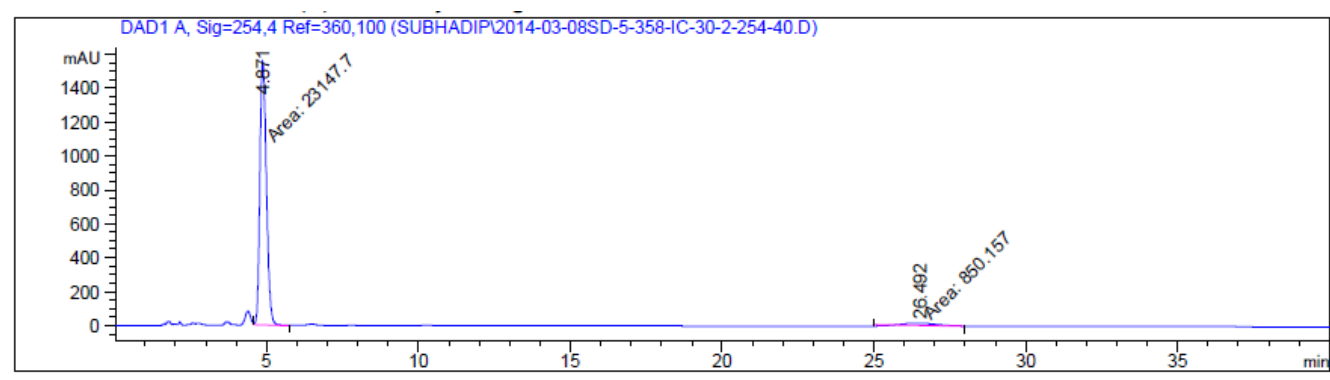

Signal 1: DAD1 A, Sig=254,4 Ref $=360,100$

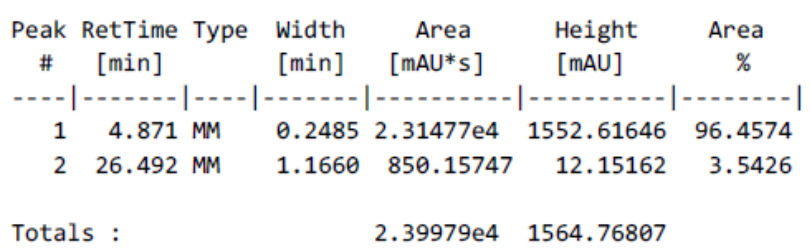

*** End of Report *** 


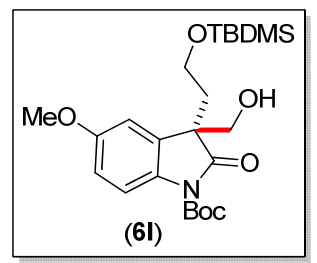

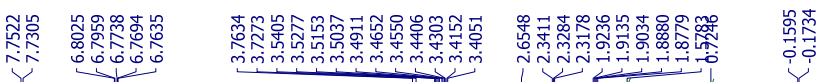

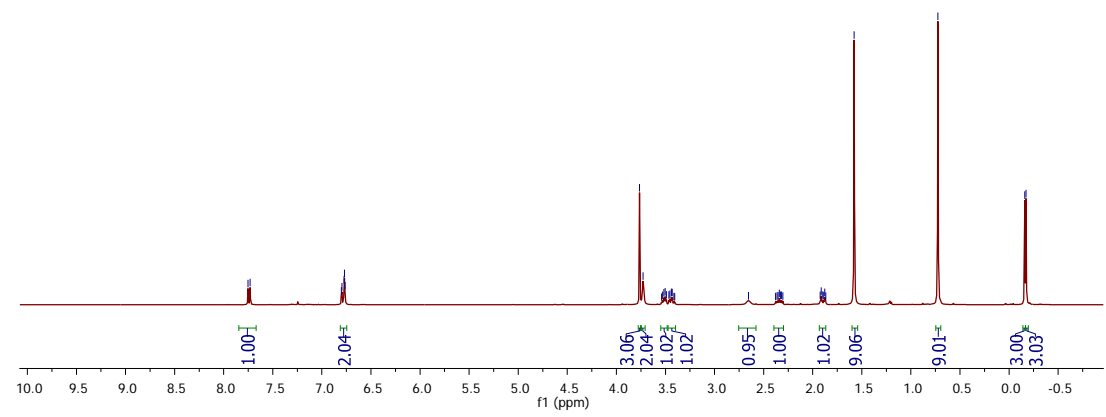

${ }^{1} \mathrm{H}$ NMR (400 MHz, $\mathrm{CDCl}_{3}$ ) of compound (6l)

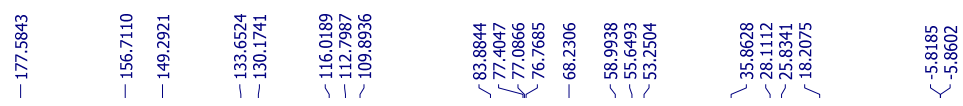

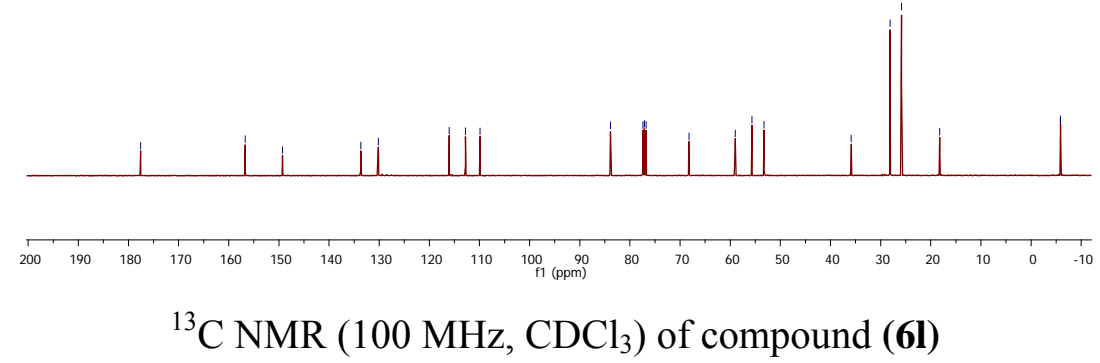




\section{Display Report}
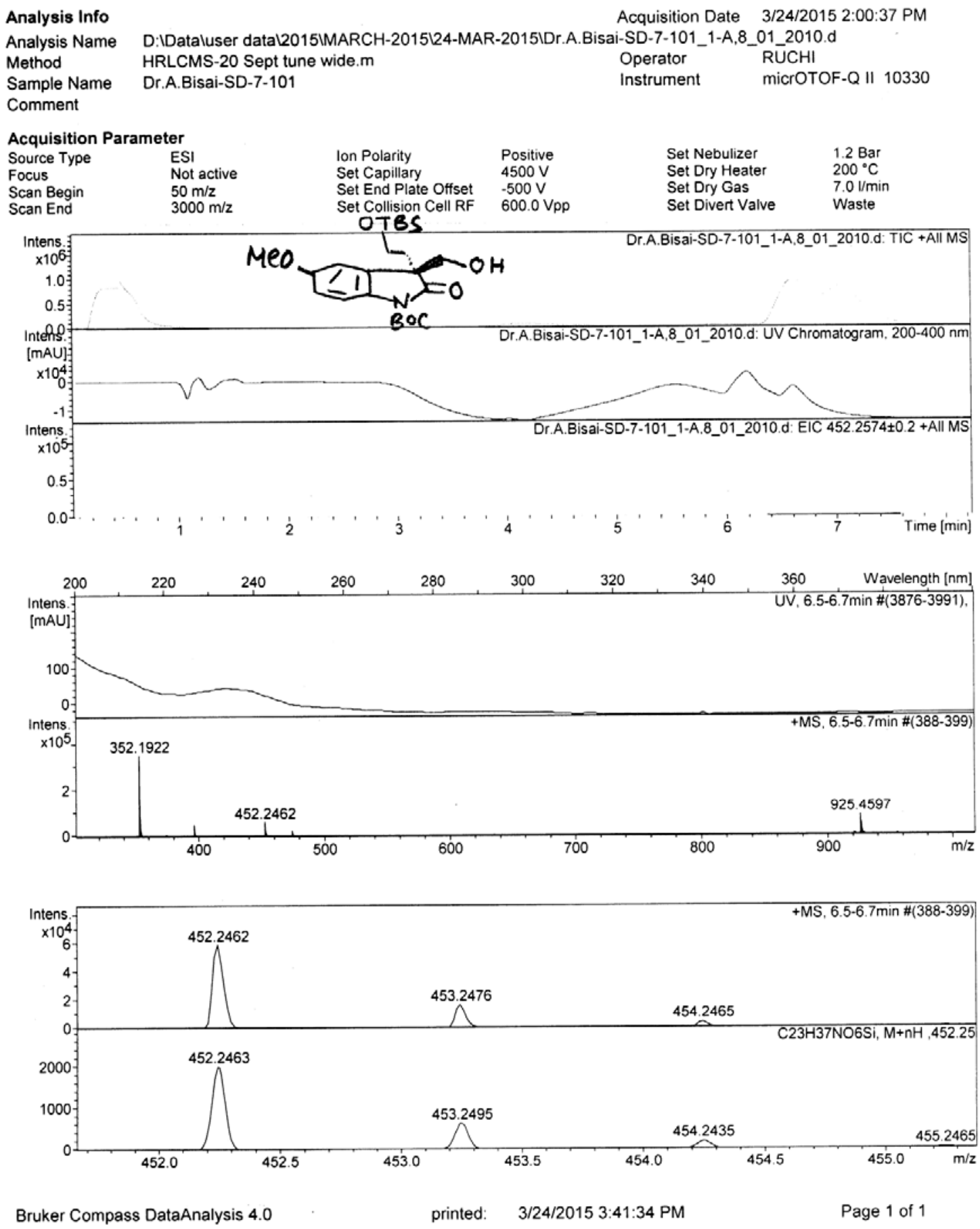

Scanned copy of mass spectrum of (6l) 


\section{HPLC data of ( \pm )-6l:}

Data File C: \CHEM32\1\DATA \SUBHADIP \2015-04-22SD-7-101_RACEMIC-IC-8-254-1-40.D Sample Name: SD-7-101_Racemic-IC-8-254-1-40

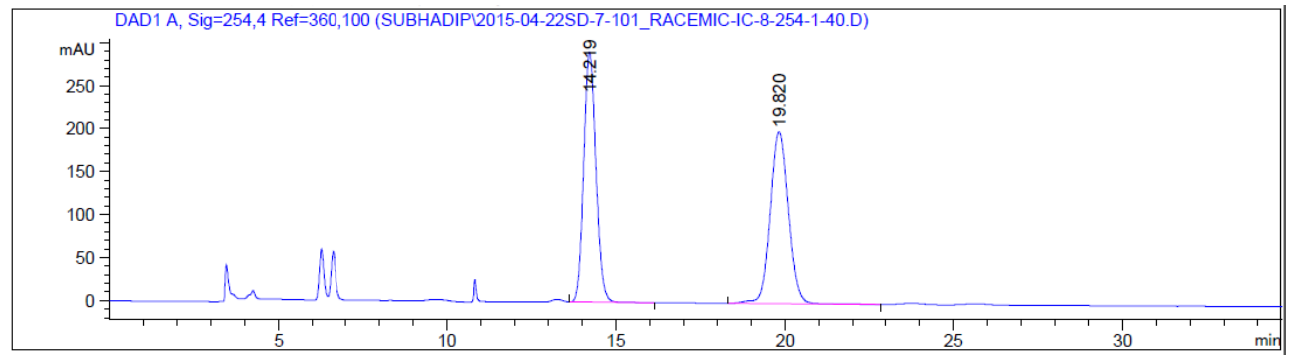

Signal 1: DAD1 A, Sig=254, 4 Ref $=360,100$

\begin{tabular}{|c|c|c|c|c|c|}
\hline $\begin{array}{c}\text { Peak } \\
\quad \#\end{array}$ & $\begin{array}{l}\text { RetTime Type } \\
\text { [min] }\end{array}$ & $\begin{array}{l}\text { Width } \\
\text { [min] }\end{array}$ & $\begin{array}{c}\text { Area } \\
{\left[\mathrm{mAU} U^{*} \mathrm{~s}\right]}\end{array}$ & $\begin{array}{l}\text { Height } \\
\text { [mAU] }\end{array}$ & $\begin{array}{c}\text { Area } \\
\%\end{array}$ \\
\hline & & & |- & $-\cdot$ & 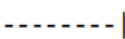 \\
\hline 1 & $14.219 \mathrm{BB}$ & 29 & 7301.55371 & 290.79889 & 49.4909 \\
\hline 2 & $20 \mathrm{BB}$ & 785 & 7451.78174 & 200.10529 & 5091 \\
\hline
\end{tabular}

Totals : $\quad 1.47533 \mathrm{e} 4 \quad 490.90417$

$* * *$ End of Report ***

\section{HPLC data of (-)-6l:}

Data File C: \CHEM32\1\DATA \MRINAL \2015-04-22MD-2-214_CHIRAL(SMALL SCALE)-IC-8-254-1-40.D Sample Name: MD-2-214_Chiral(small scale)-IC-8-254-1-

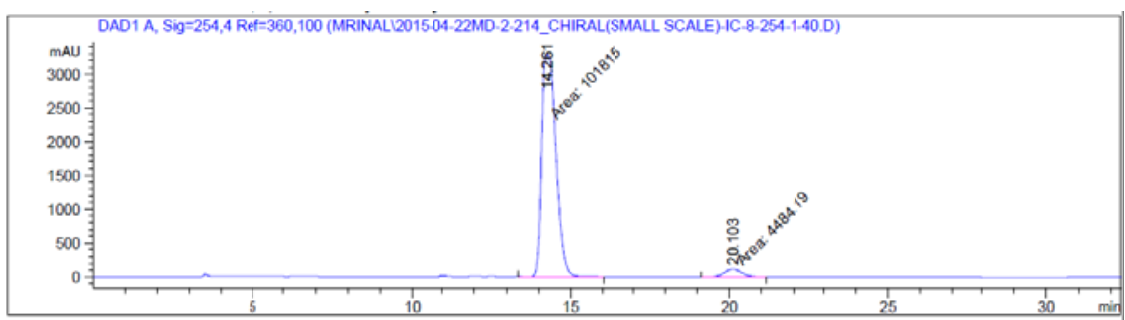

Signal 1: DAD1 A, Sig=254,4 Ref $=360,100$

\begin{tabular}{cccccc}
$\begin{array}{c}\text { Peak RetTime Type } \\
\text { \# }\end{array}\left[\begin{array}{c}\text { Width } \\
{[\mathrm{min}]}\end{array}\right.$ & $\begin{array}{c}\text { Area } \\
{[\mathrm{mAU} \text { ( }]}\end{array}$ & $\begin{array}{c}\text { Height } \\
{[\mathrm{mAU}]}\end{array}$ & $\begin{array}{c}\text { Area } \\
\%\end{array}$ \\
\hline 1 & $14.261 \mathrm{MM}$ & 0.5148 & $1.01815 \mathrm{e} 5$ & 3296.28076 & 95.7816 \\
2 & $20.103 \mathrm{MM}$ & 0.6169 & 4484.18604 & 121.13975 & 4.2184
\end{tabular}

Totals : $\quad 1.06300 \mathrm{e} 5 \quad 3417.42051$ 

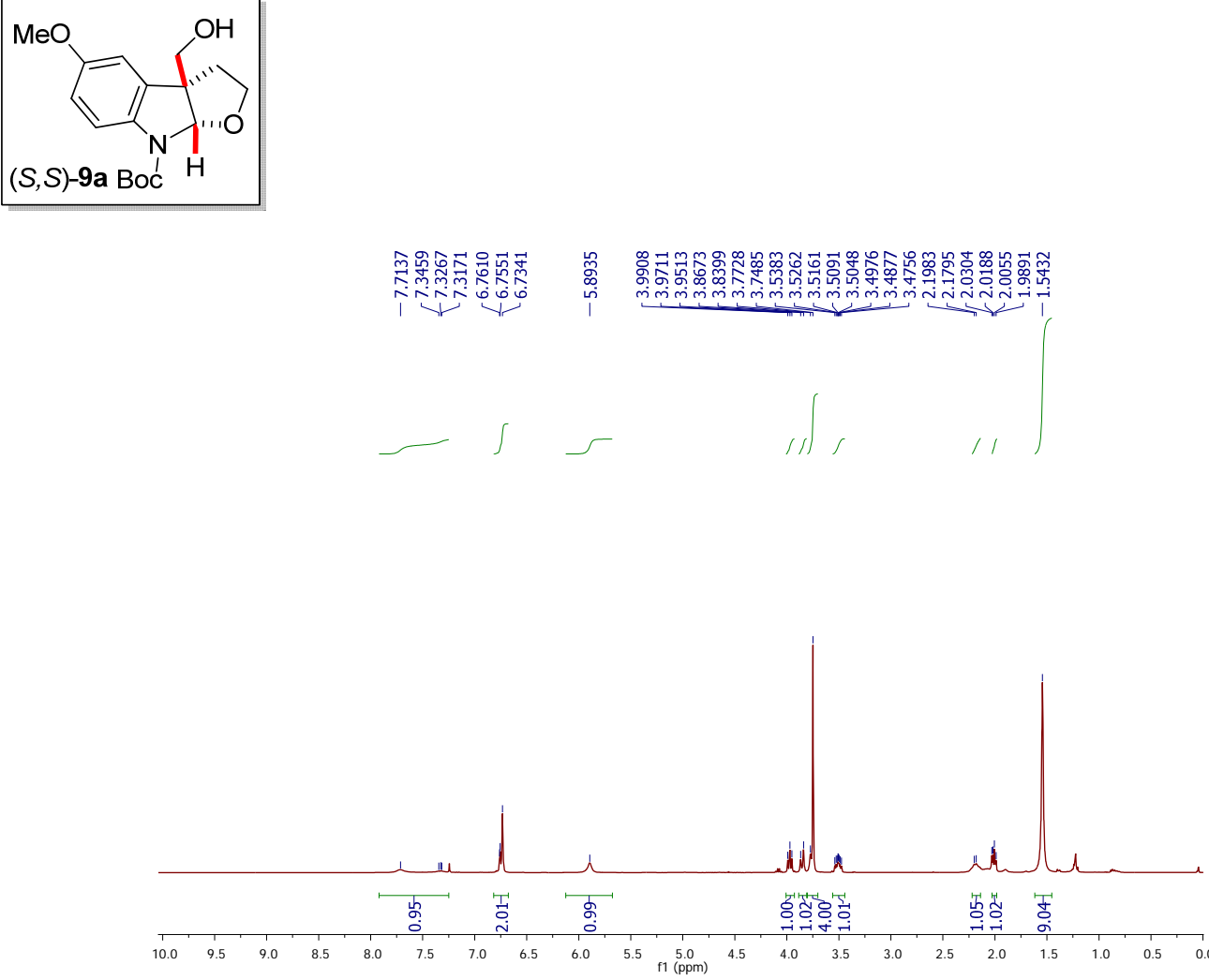

${ }^{1} \mathrm{H}$ NMR (400 MHz, $\mathrm{CDCl}_{3}$ ) of compound (9a)

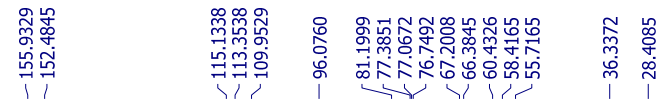

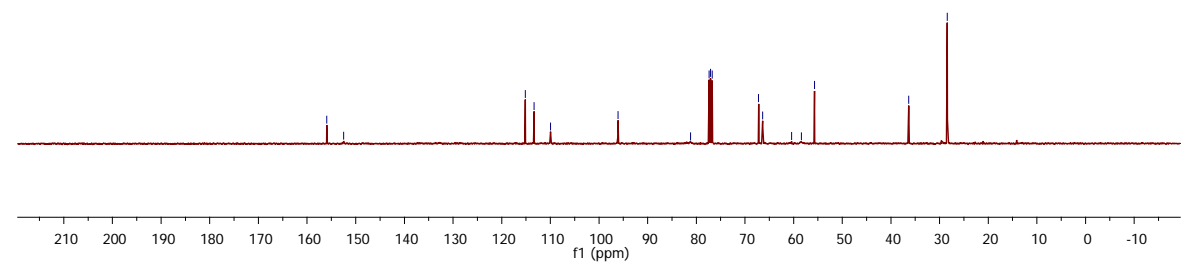

${ }^{13} \mathrm{C}$ NMR (100 MHz, $\mathrm{CDCl}_{3}$ ) of compound (9a) 


\section{Display Report}
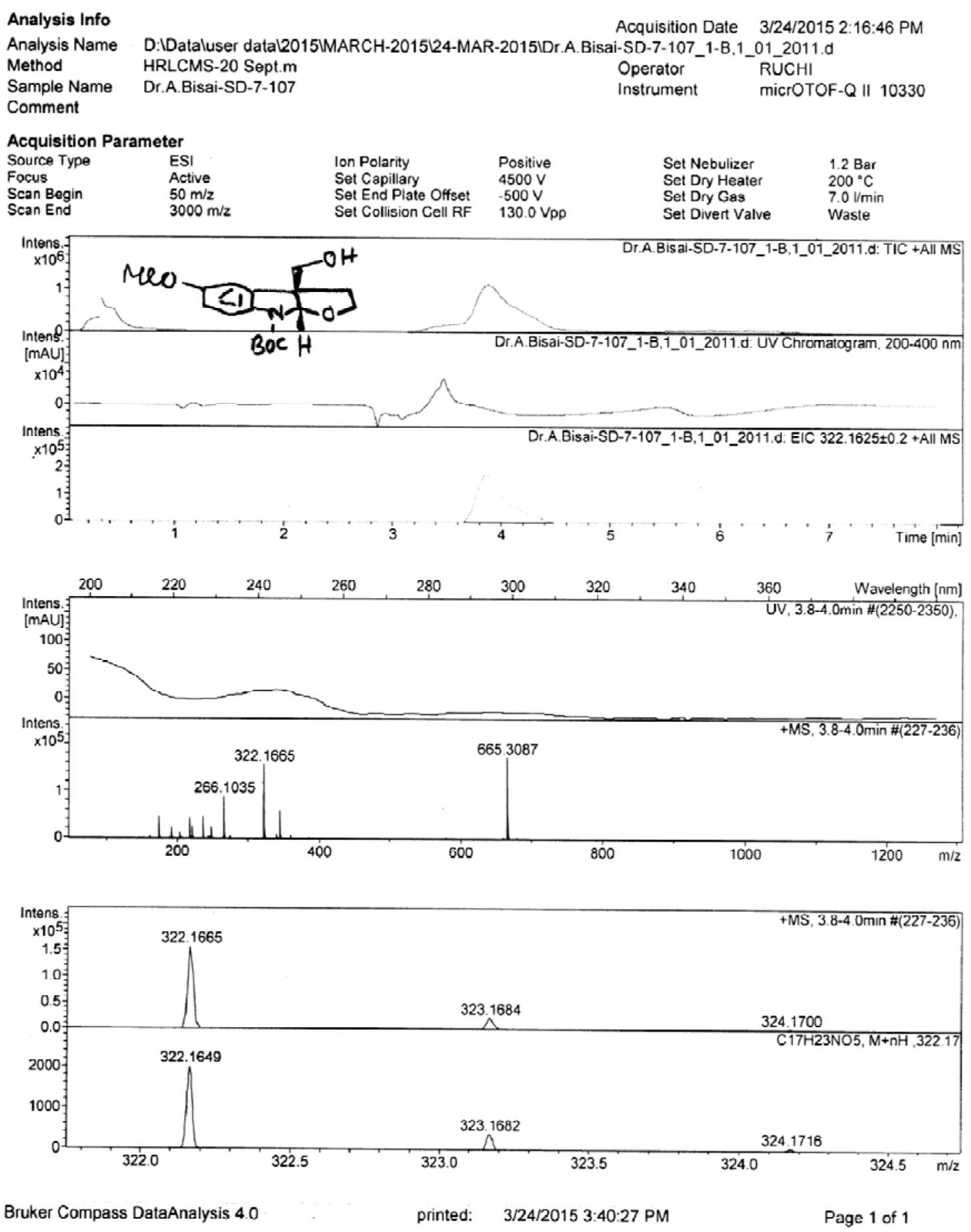

Scanned copy of mass spectrum of (9a) 


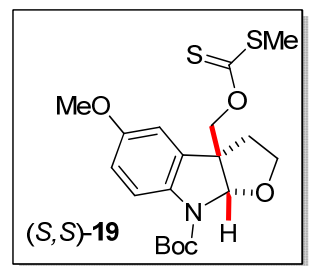

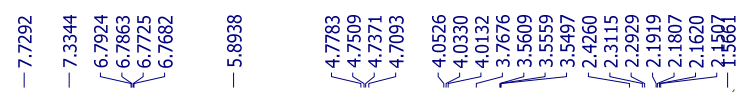
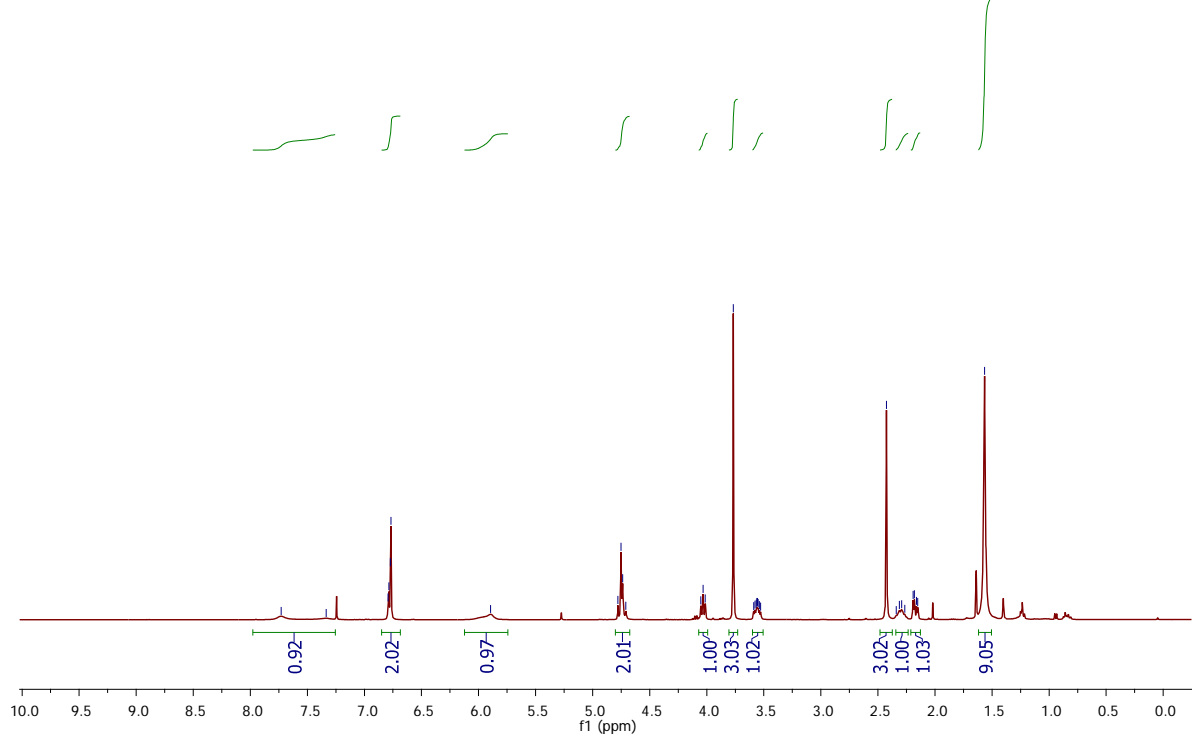

${ }^{1} \mathrm{H}$ NMR (400 MHz, $\mathrm{CDCl}_{3}$ ) of compound (19)

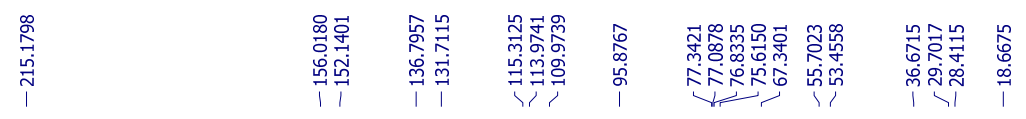

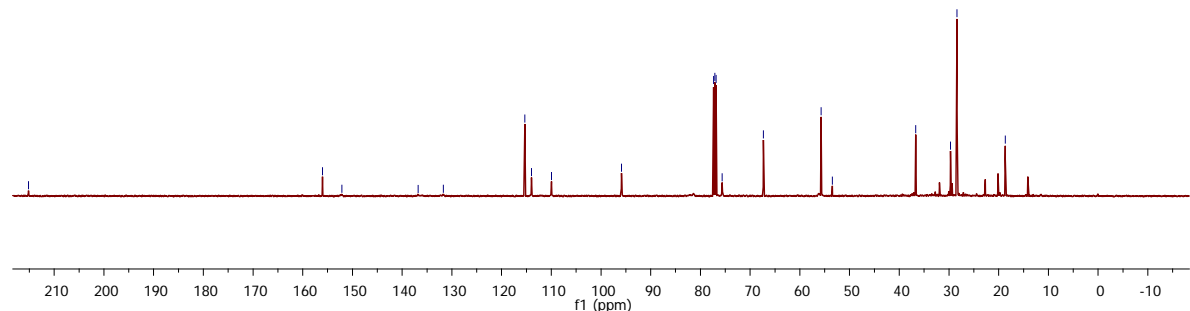

${ }^{13} \mathrm{C} \mathrm{NMR}\left(1219 \mathrm{MHz}, \mathrm{CDCl}_{3}\right)$ of compound (19) 


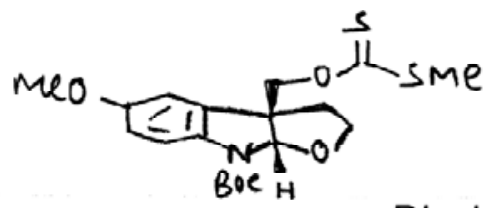

\section{Display Report}
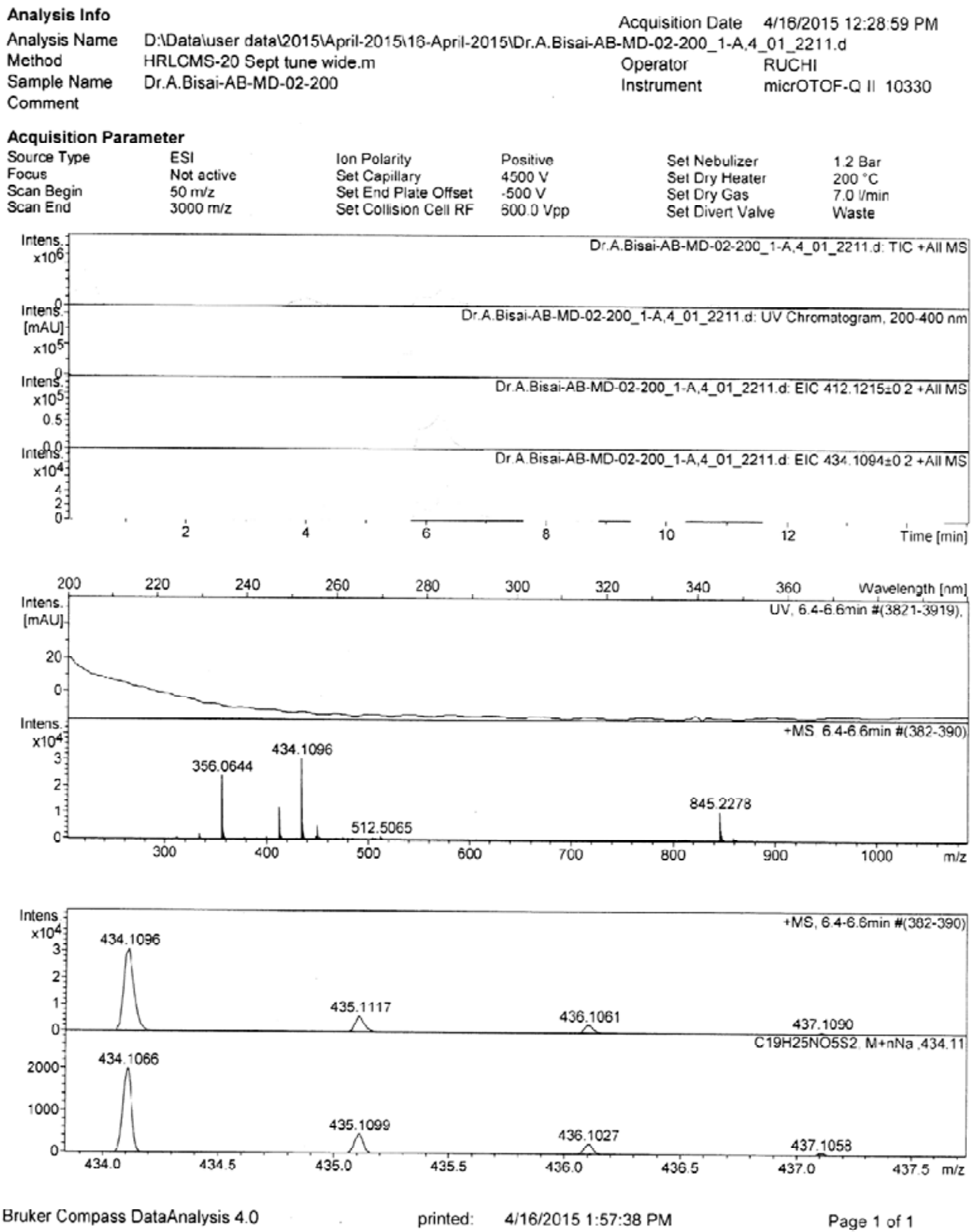

Scanned copy of mass spectrum of (19) 


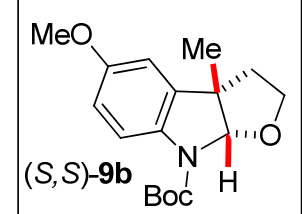

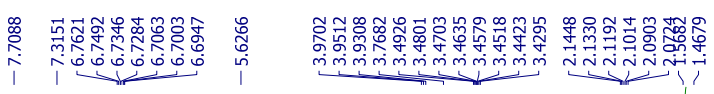

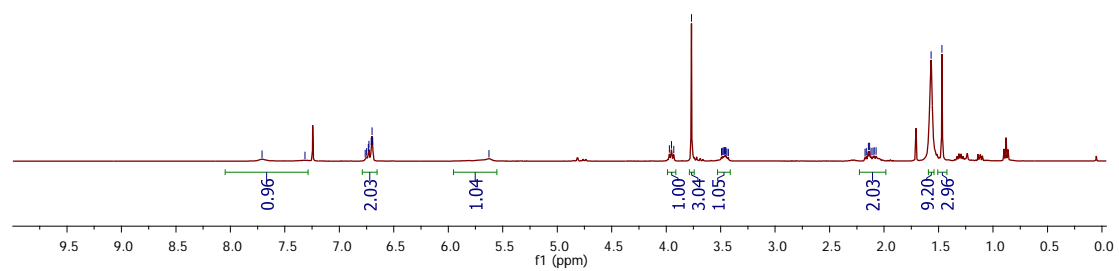

${ }^{1} \mathrm{H}$ NMR (400 MHz, $\mathrm{CDCl}_{3}$ ) of compound (9b)

\begin{tabular}{|c|c|c|c|c|c|}
\hline 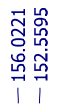 & 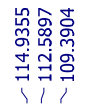 & 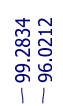 & 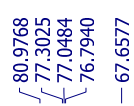 & 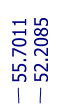 & । \\
\hline
\end{tabular}

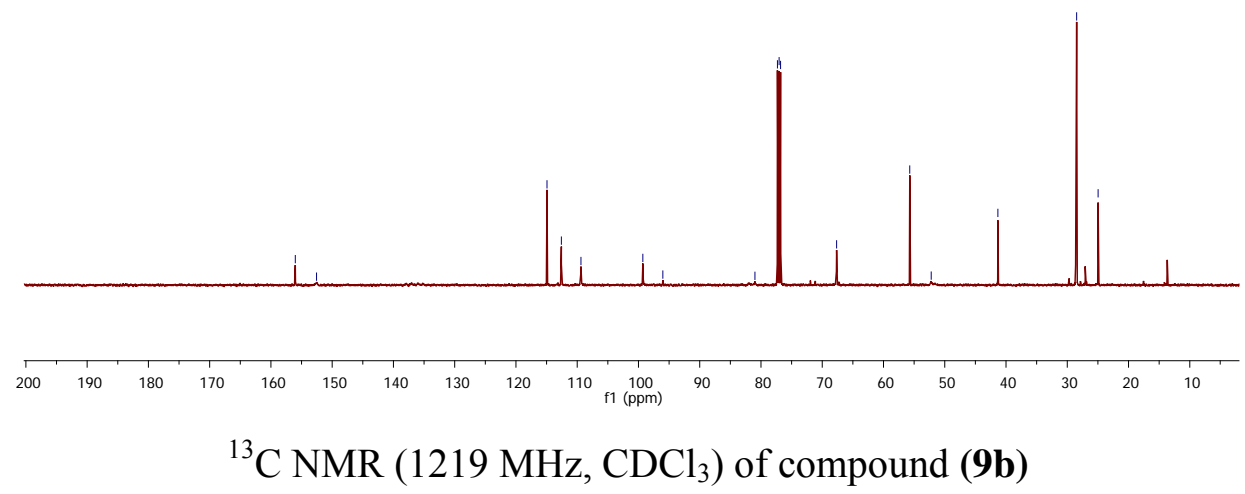




\section{Display Report}

Analysis Info

Analysis Name

Method

Sample Name

Comment

Acquisition Date 4/16/2015 12:43:51 PM

D:IDataluser dataL2015 April-2015\16-April-2015IDr.A.Bisai-AB-MD-02-202_1-A,5_01_2212.d

HRLCMS.20 Sept.m

Operator - RUCHI

Dr.A.Bisai-AB-MD-02-202

Instrument micrOTOF-Q II 10330
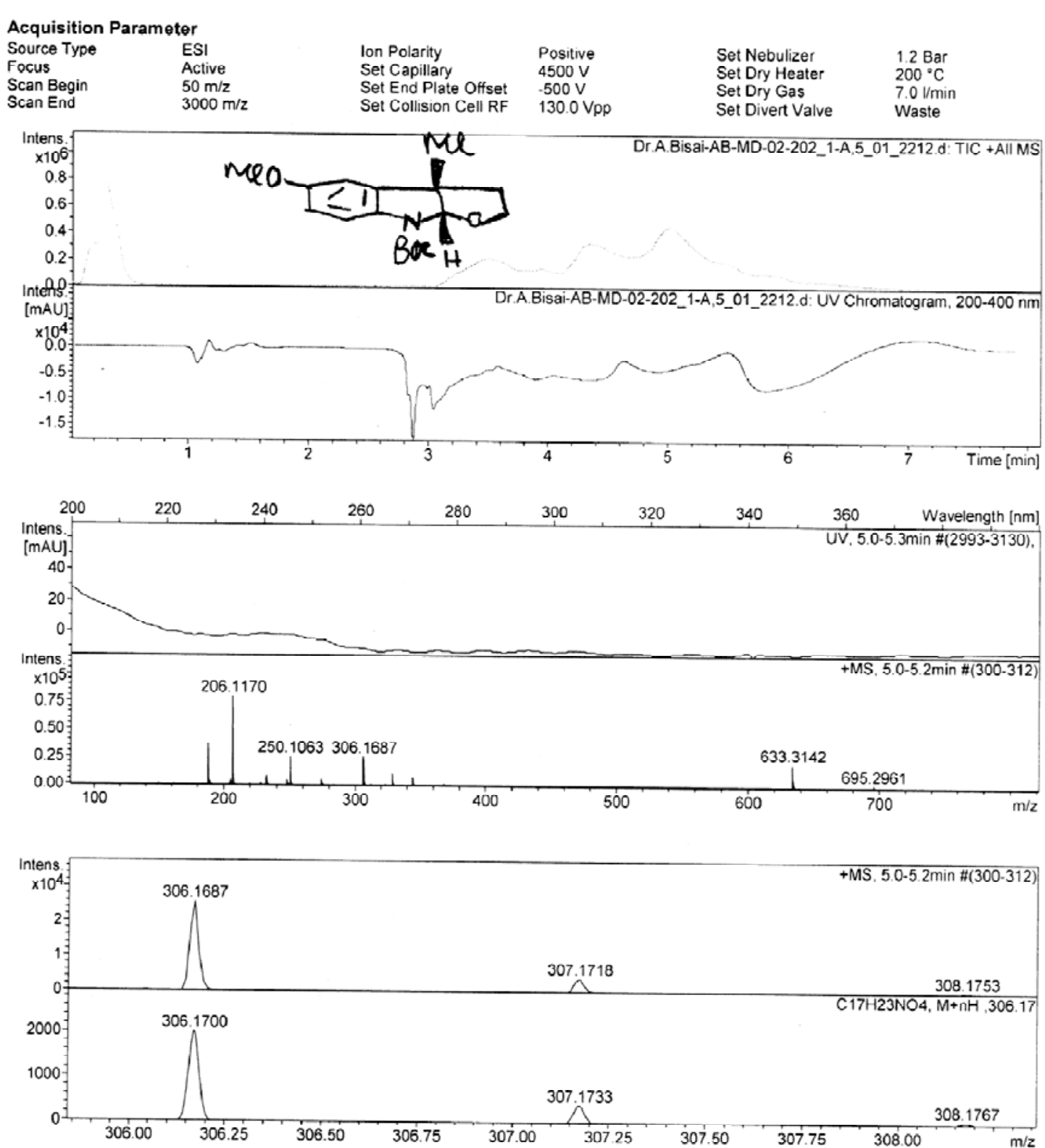

Bruker Compass DataAnalysis 4.0

printed: $\quad 4 / 17 / 201511: 34: 42 \mathrm{AM}$

Page 1 of 1

Scanned copy of mass spectrum of $(\mathbf{9 b})$ 

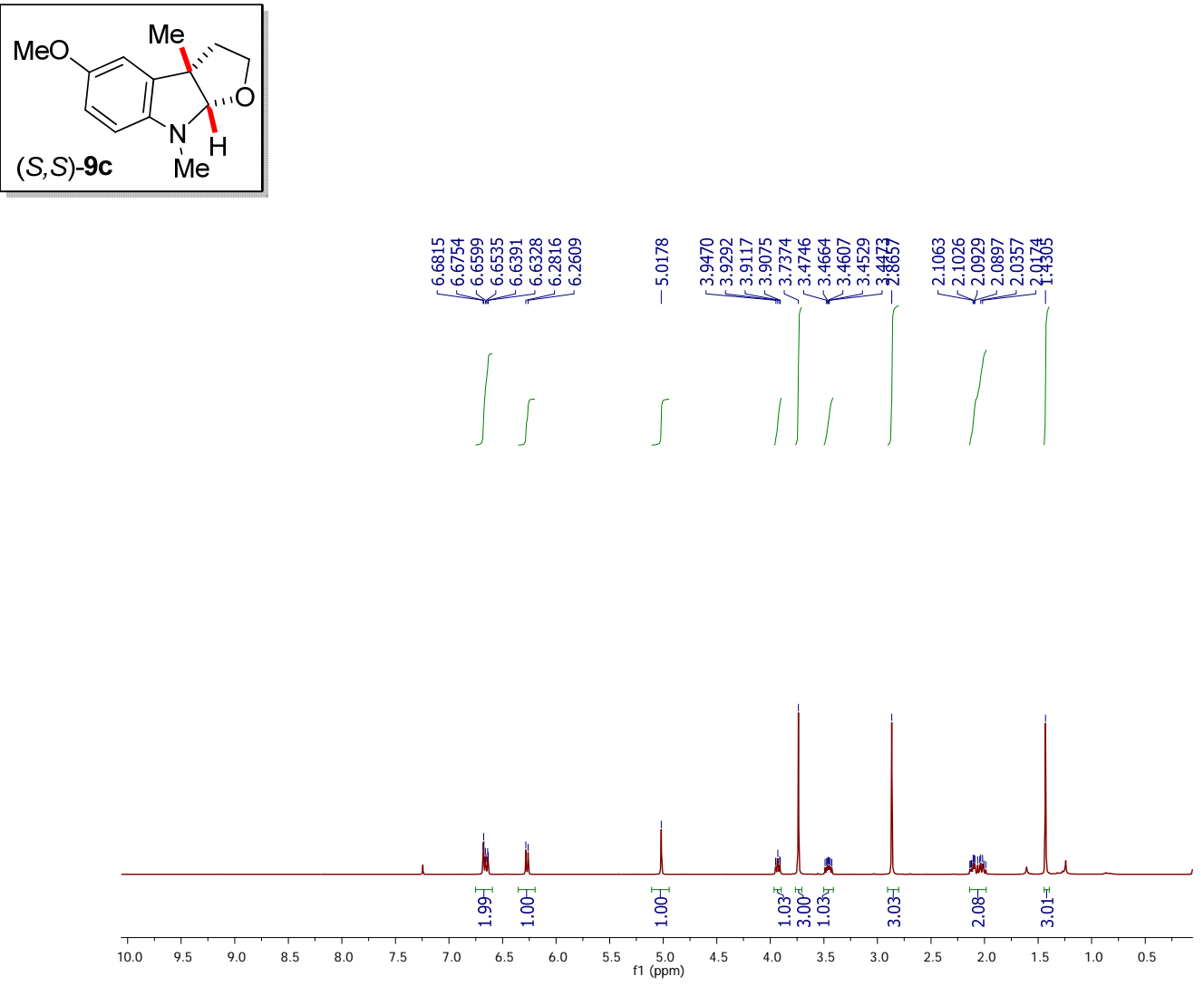

${ }^{1} \mathrm{H}$ NMR (400 MHz, $\mathrm{CDCl}_{3}$ ) of compound (9c)

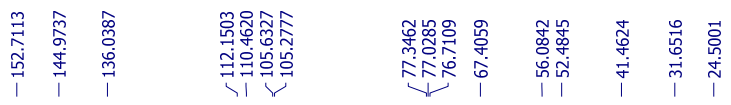

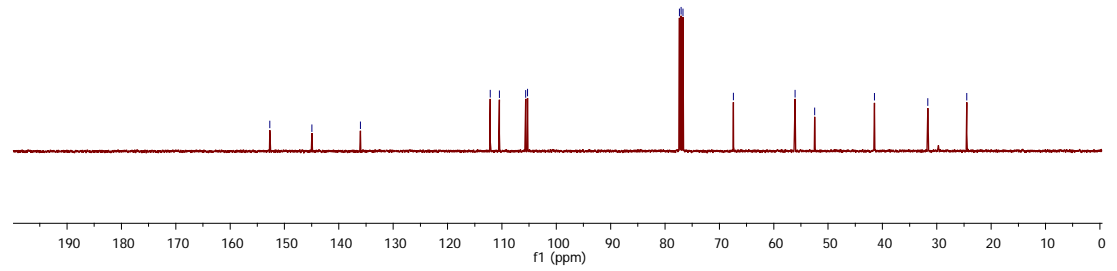

${ }^{13} \mathrm{C}$ NMR (100 MHz, $\mathrm{CDCl}_{3}$ ) of compound (9c) 
Display Report

Analysis Info

Analysis Name

Method

Sample Name

Comment

\section{Acquisition Parameter}

Focus

Scan Begin

Scan End

Active

$50 \mathrm{~m} / \mathrm{z}$

$3000 \mathrm{~m} / 2$

Acquisition Date $\quad 4 / 22 / 2015$ 11:55:17 AM

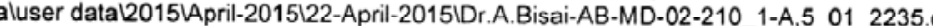

Operator $\quad \mathrm{RU} \mathrm{CHI}$

Instrument micrOTOF-Q || 10330
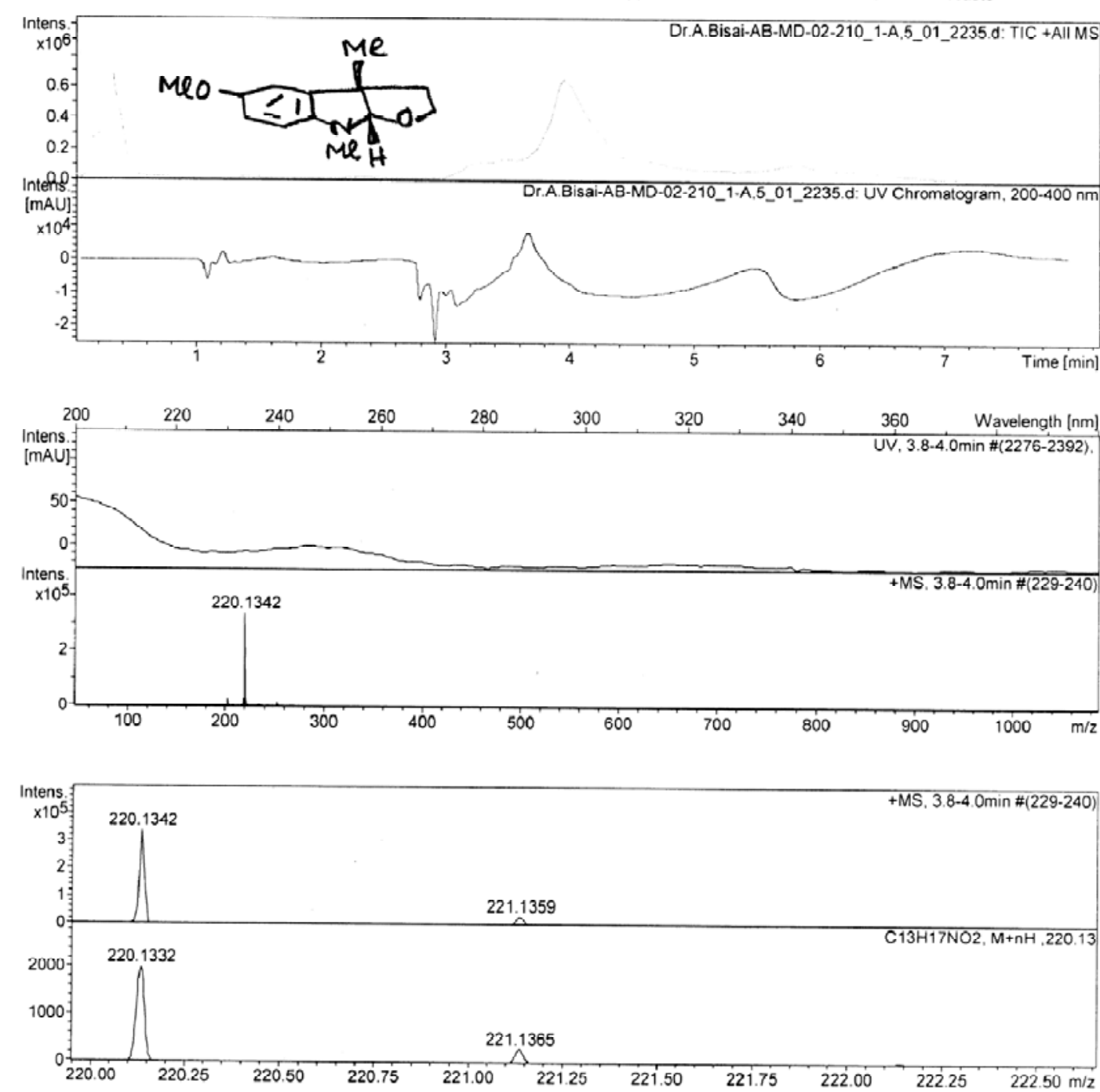

Bruker Compass DataAnalysis 4.0

printed: $\quad 4 / 22 / 2015$ 12:46:12 PM

Page 1 of 1

Scanned copy of mass spectrum of $(\mathbf{9 c})$ 


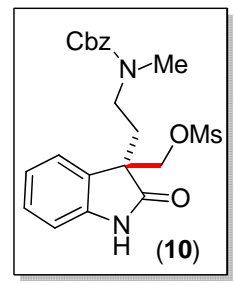

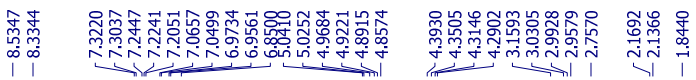
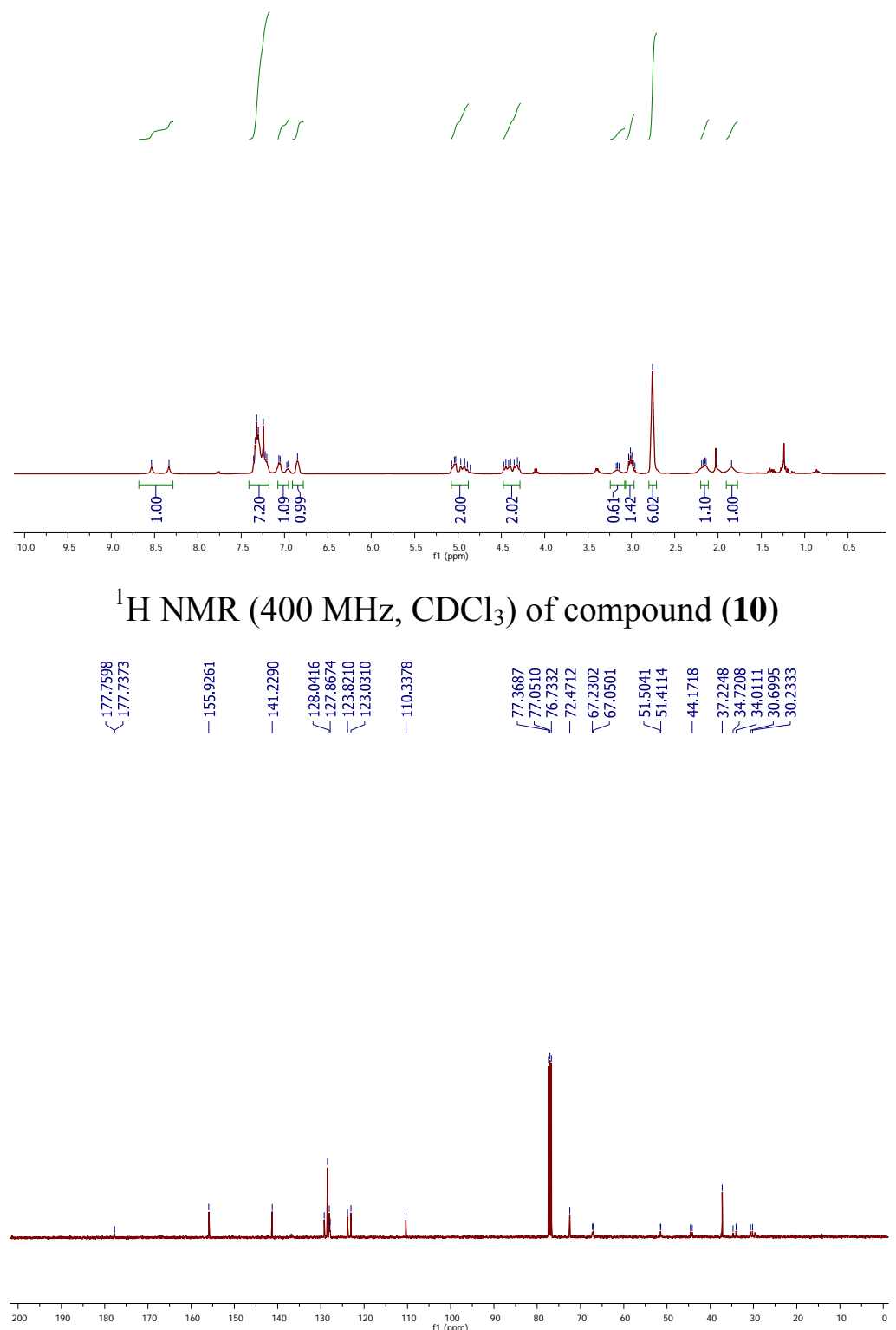

${ }^{13} \mathrm{C}$ NMR (100 MHz, $\mathrm{CDCl}_{3}$ ) of compound (10) 


\section{Display Report}

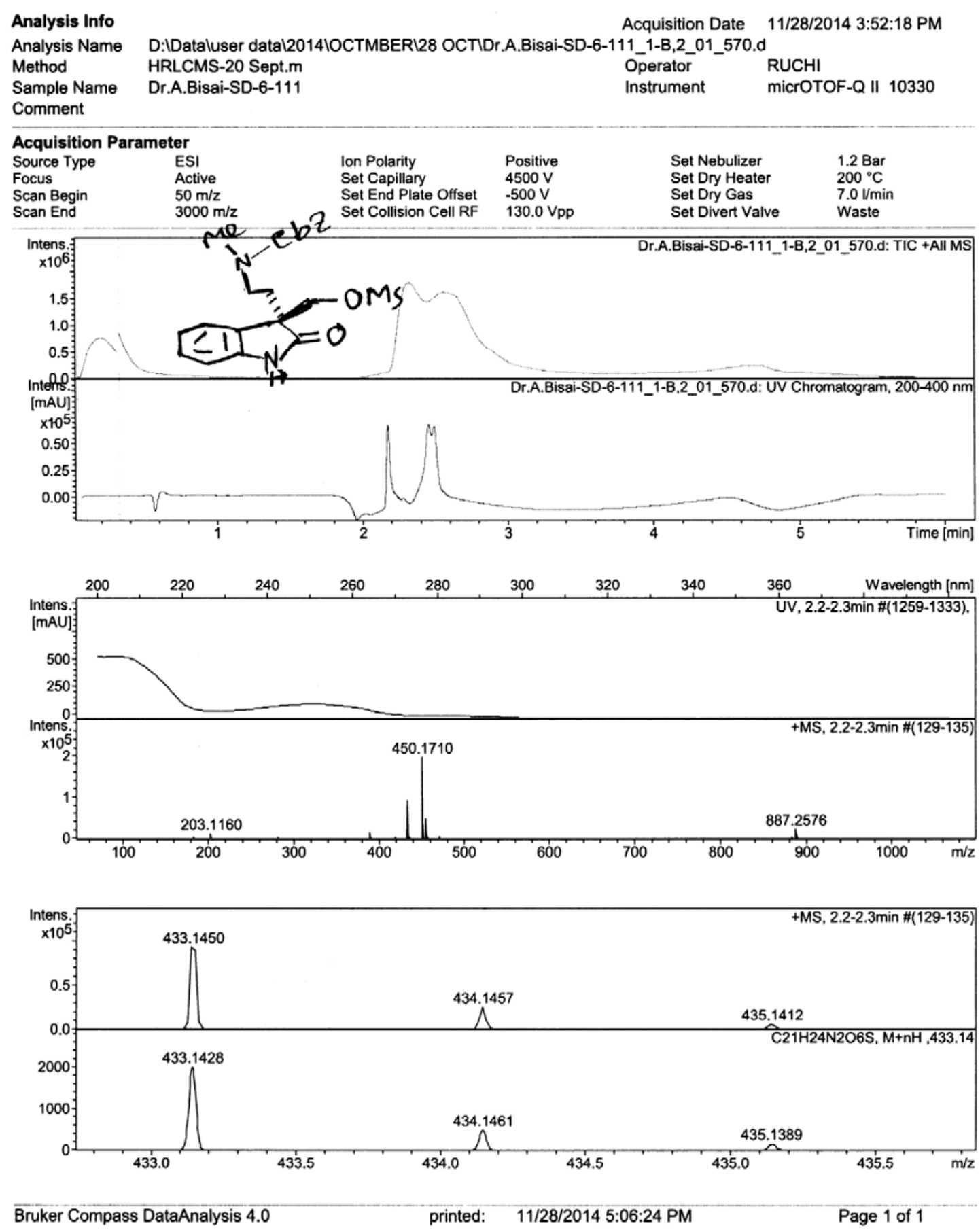

Scanned copy of mass spectrum of (10) 


\section{Display Report}
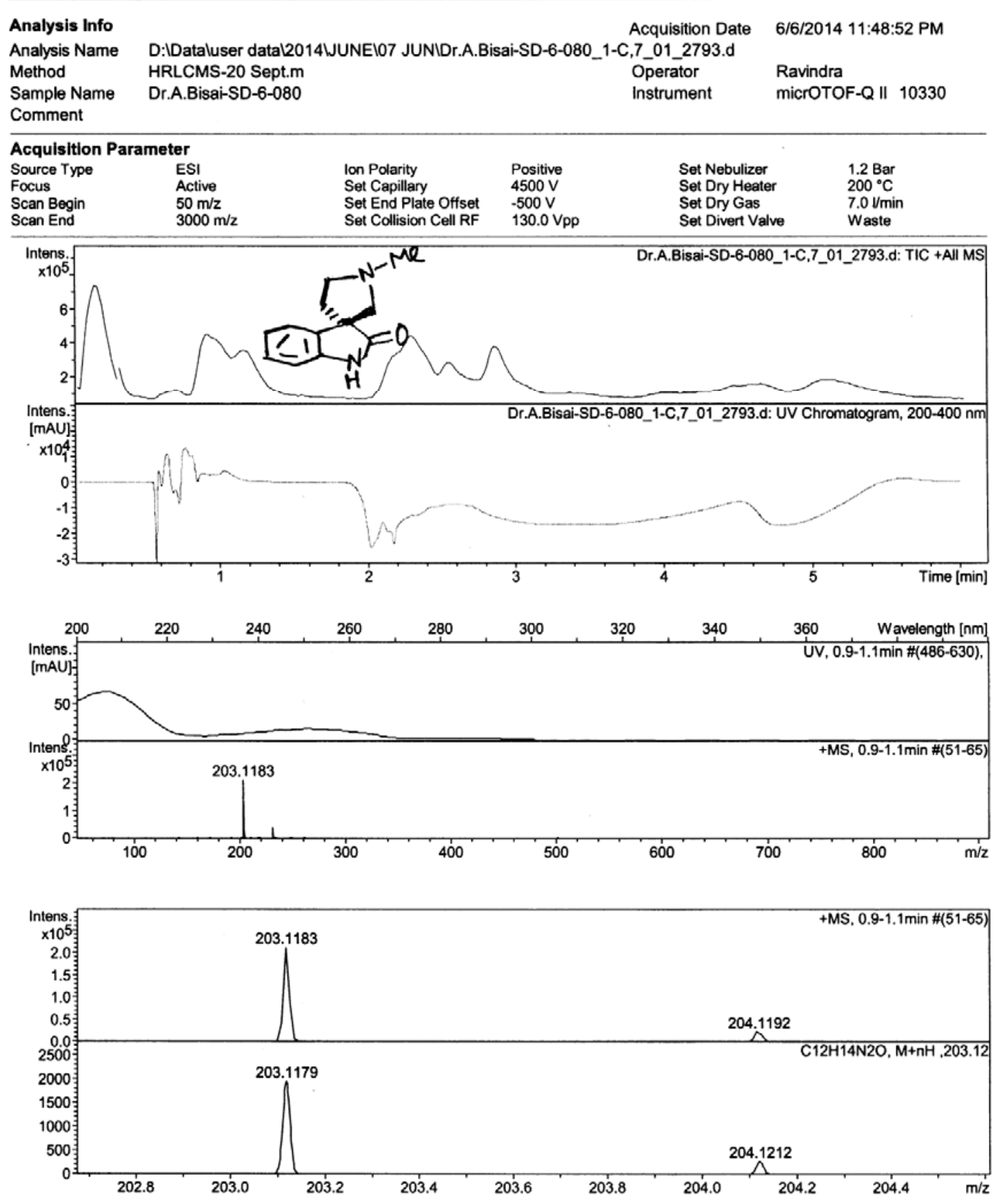

Bruker Compass DataAnalysis 4.0

printed: $\quad 6 / 7 / 2014$ 12:19:42 AM

Page 1 of 1

Scanned copy of mass spectrum of (1a) 


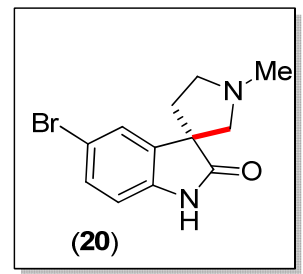

营

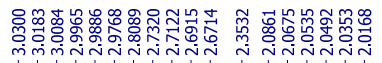
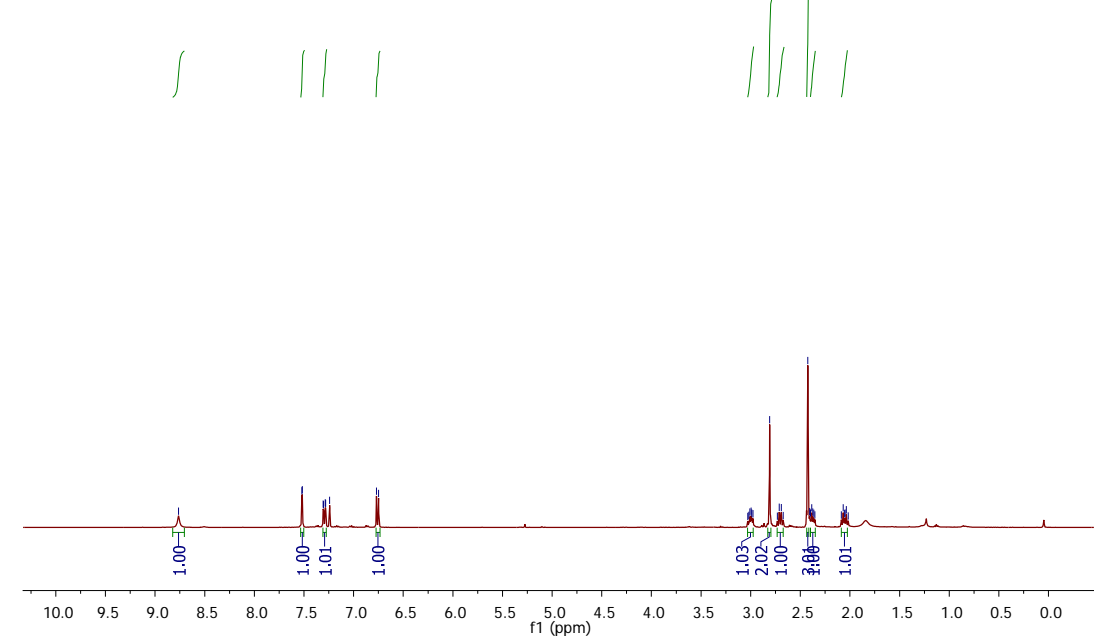

${ }^{1} \mathrm{H}$ NMR (400 MHz, $\mathrm{CDCl}_{3}$ ) of compound (20)

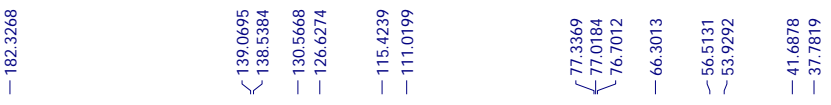

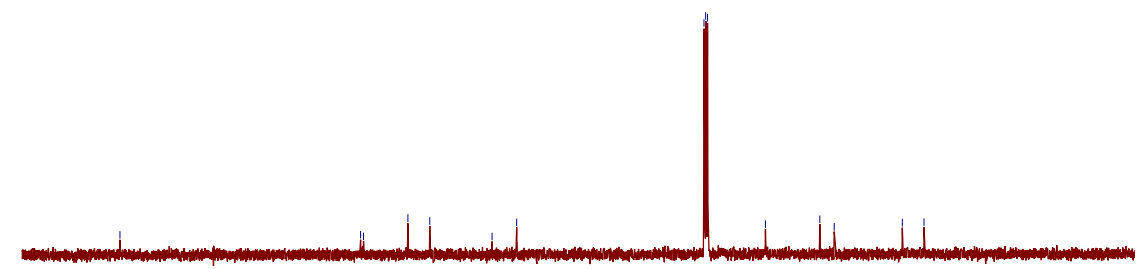

$\begin{array}{lllllllllllllllllllll}190 & 180 & 170 & 160 & 150 & 140 & 130 & 120 & 110 & \begin{array}{l}100 \\ \mathrm{f} 1(\mathrm{ppm})\end{array} & 90 & 80 & 70 & 60 & 50 & 40 & 30 & 20 & 10\end{array}$

${ }^{13} \mathrm{C}$ NMR (100 MHz, $\mathrm{CDCl}_{3}$ ) of compound (20) 


\section{Display Report}
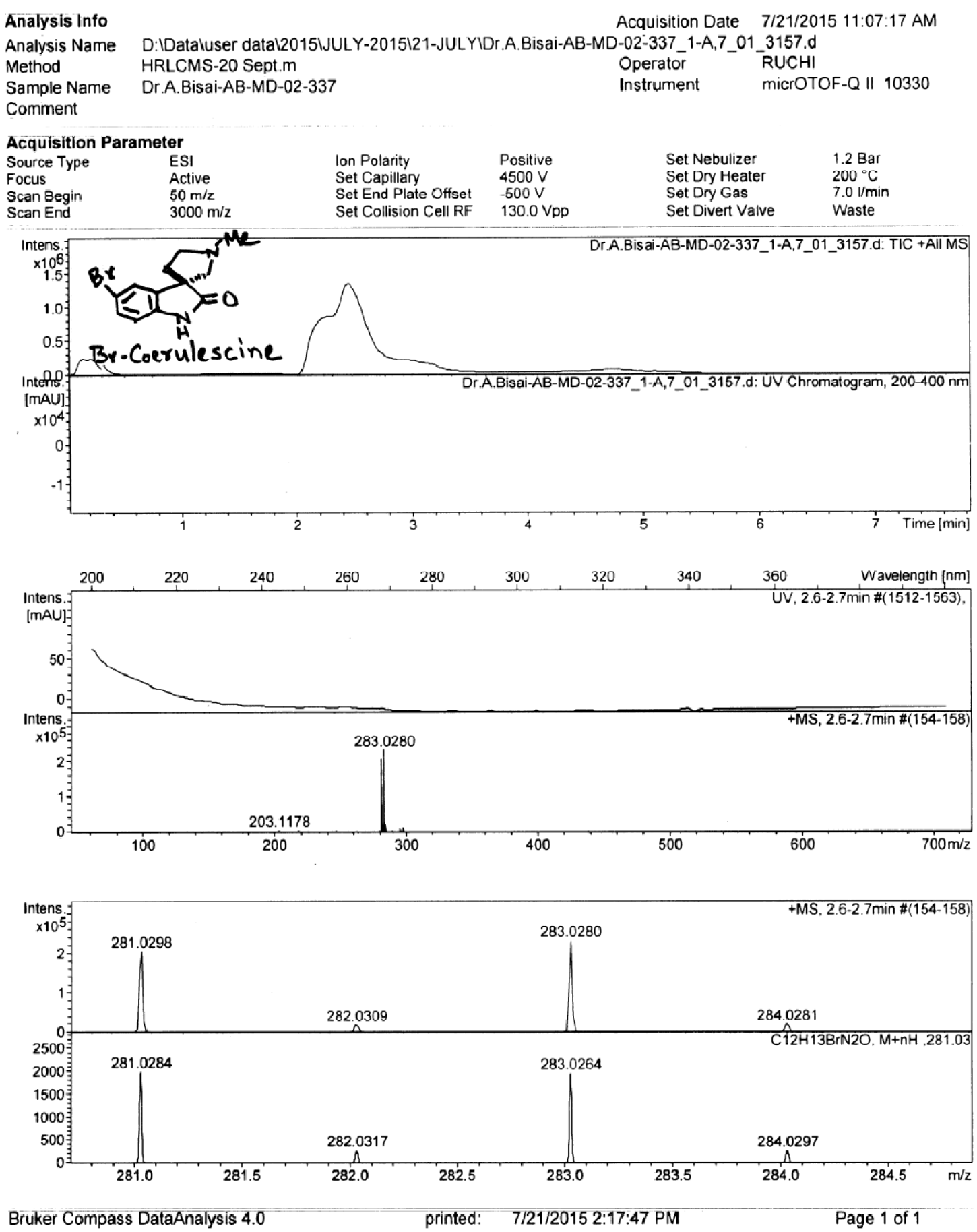

Scanned copy of mass spectrum of (20) 

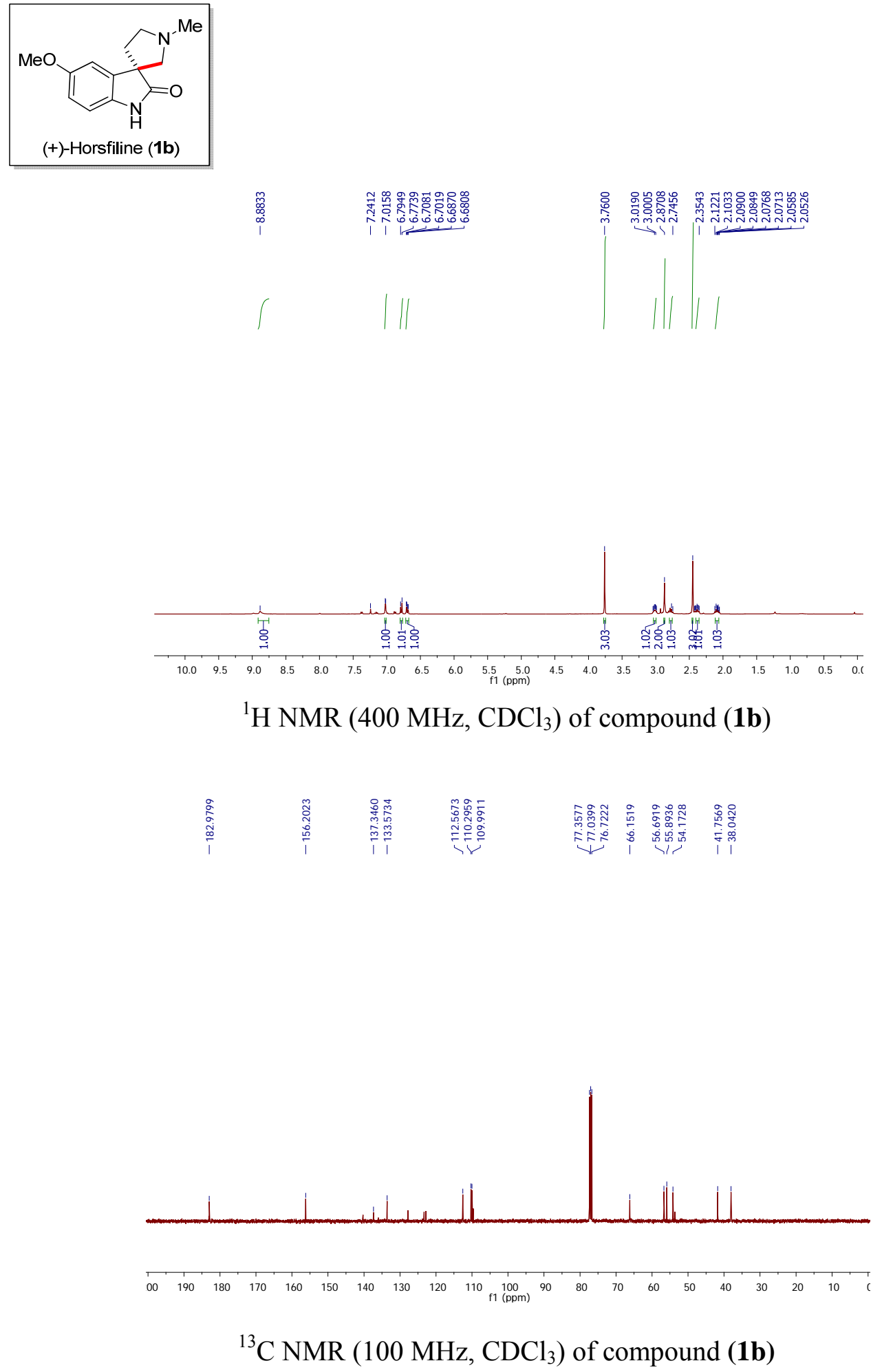


\section{Display Report}
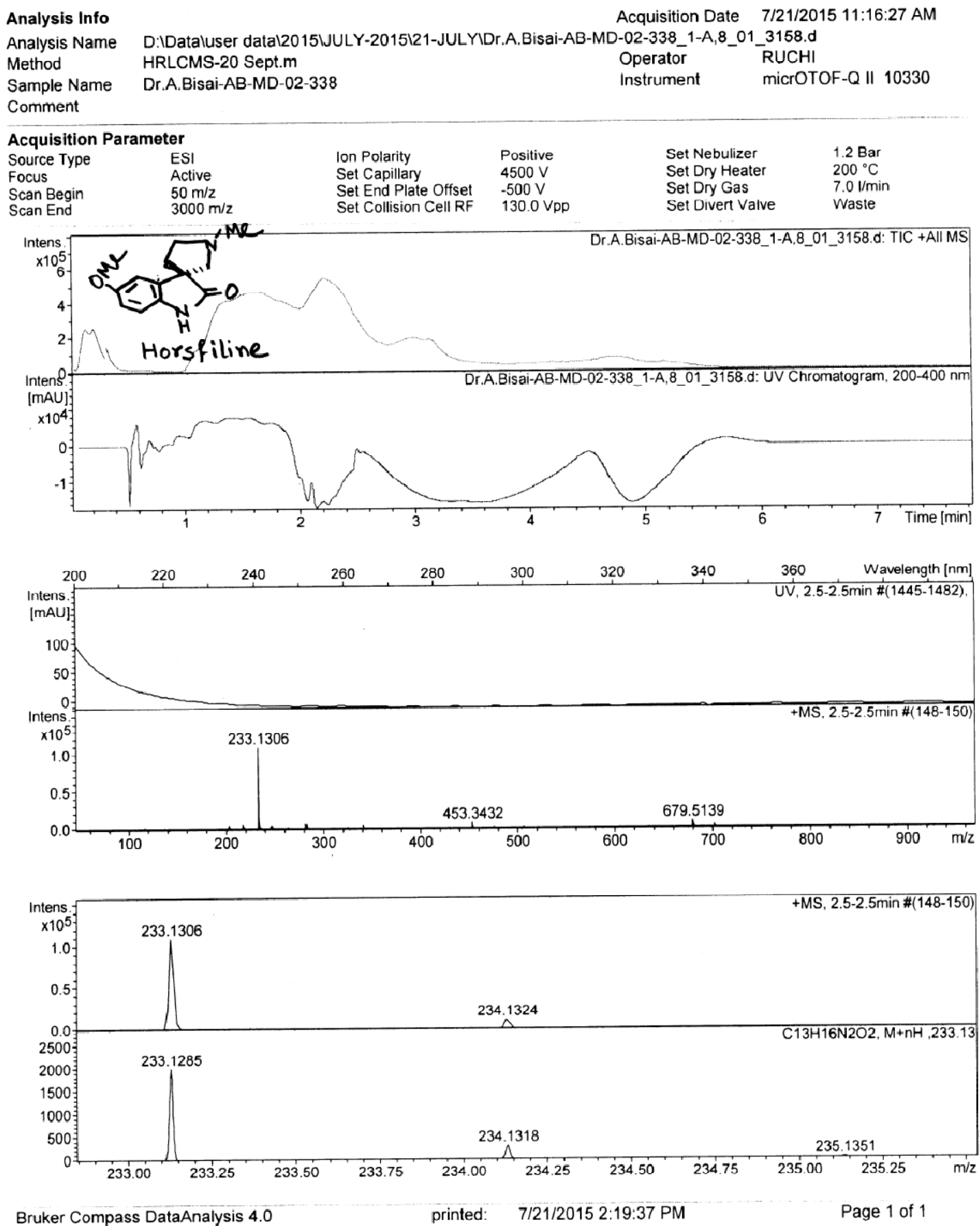

Scanned copy of mass spectrum of (1b) 


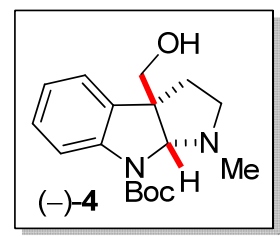

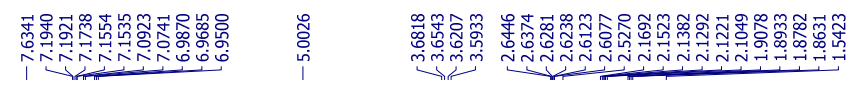

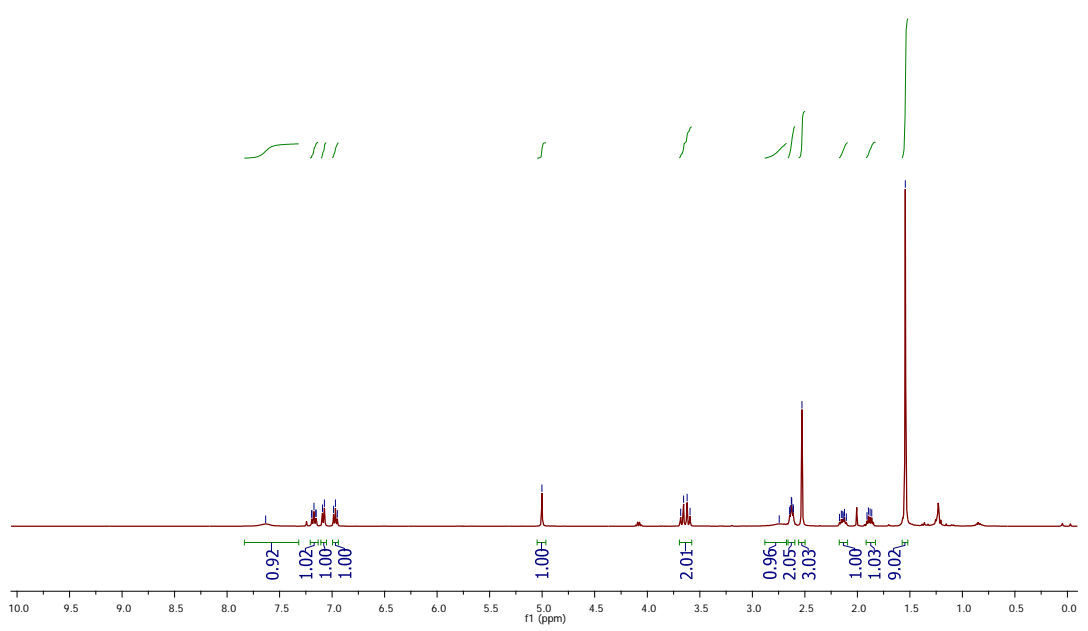

${ }^{1} \mathrm{H}$ NMR (400 MHz, $\mathrm{CDCl}_{3}$ ) of compound (4)

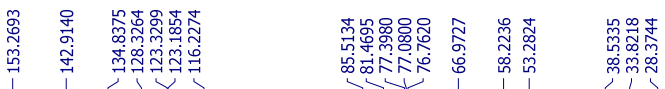

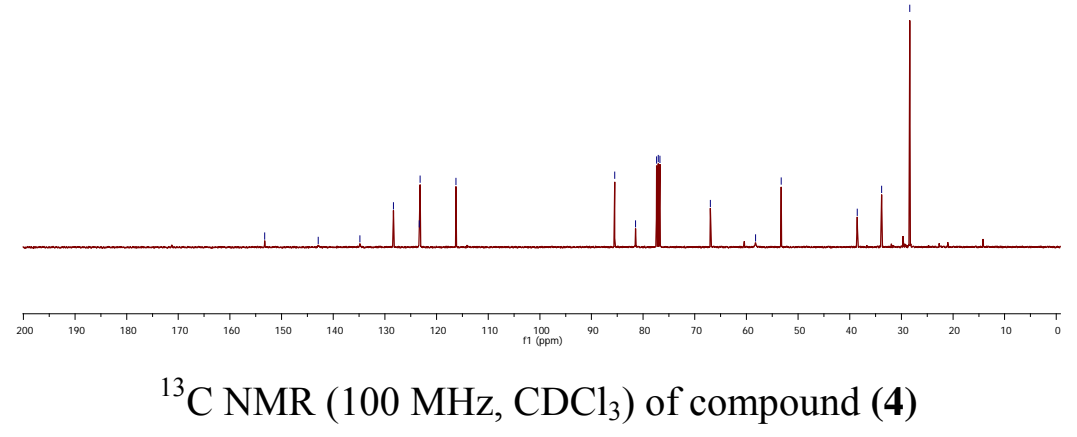



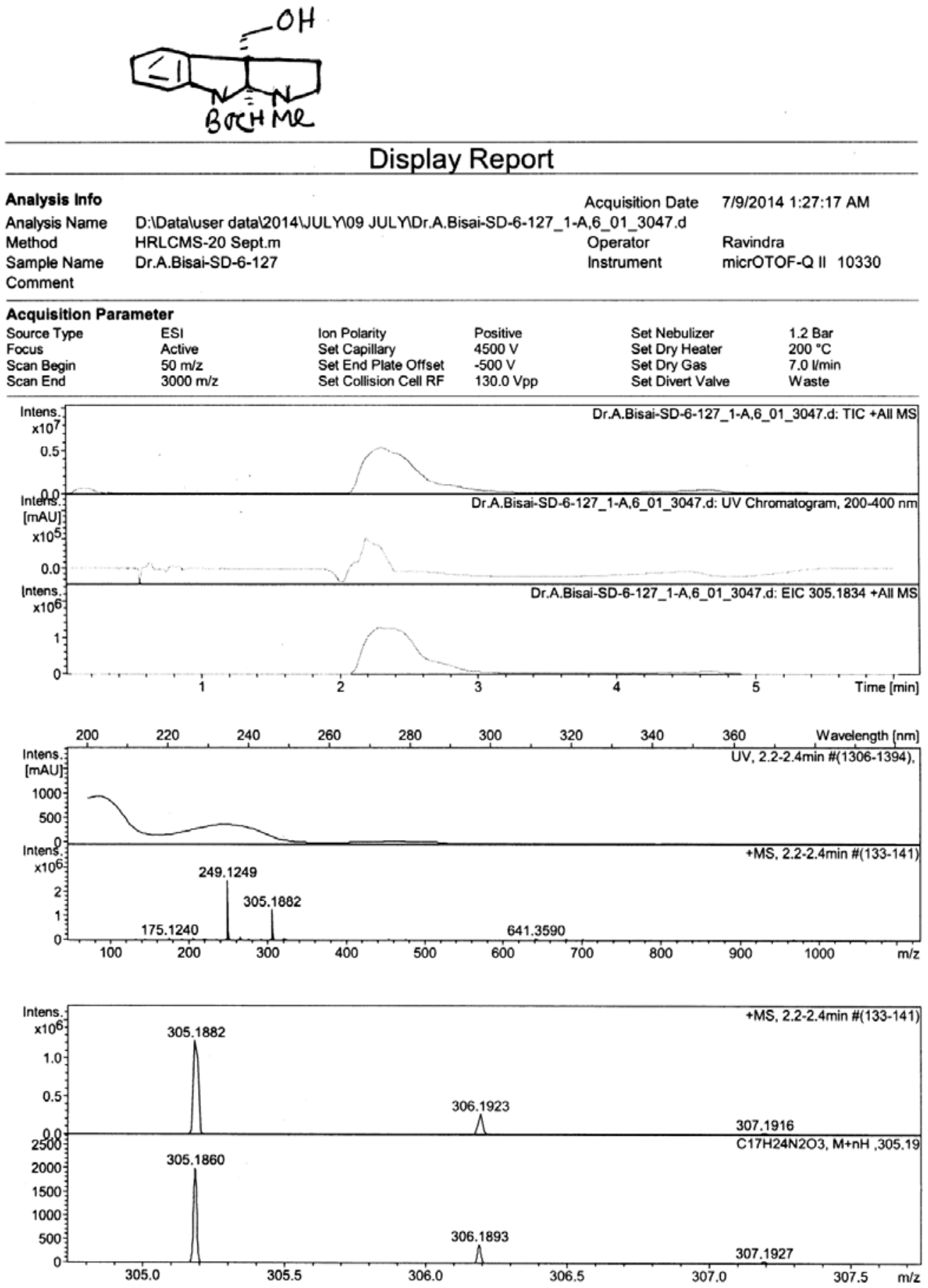

Scanned copy of mass spectrum of (4) 


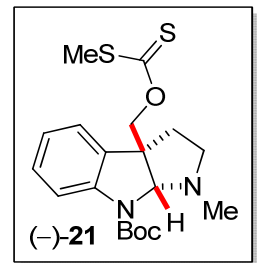

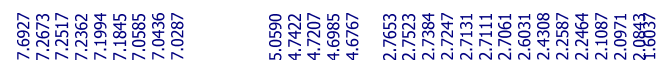

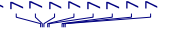
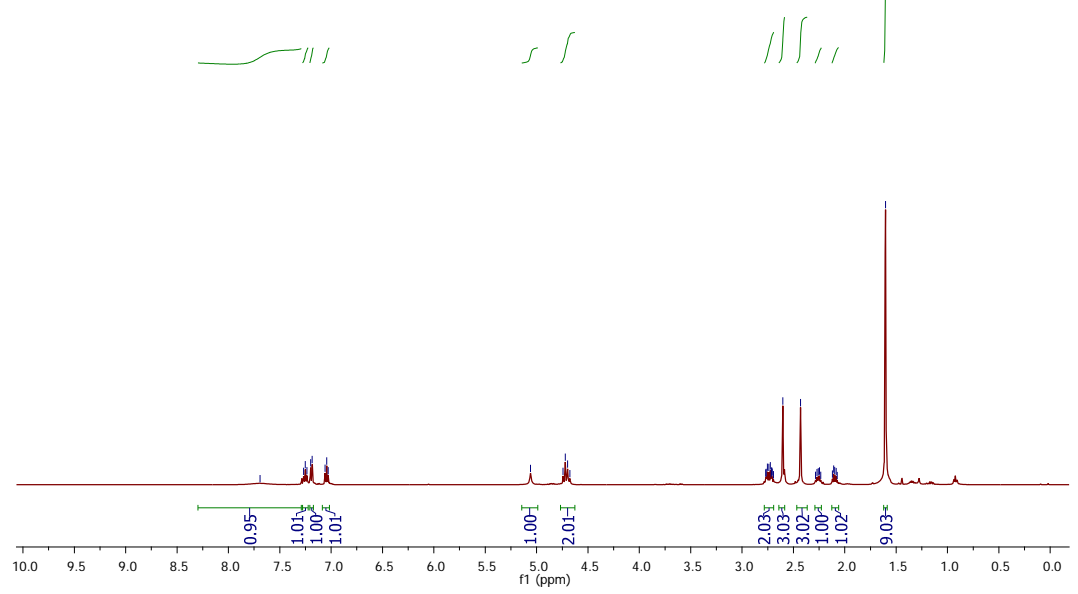

${ }^{1} \mathrm{H}$ NMR (1900 MHz, $\mathrm{CDCl}_{3}$ ) of compound (21)

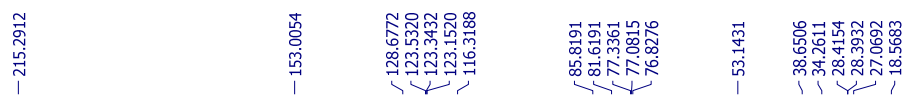

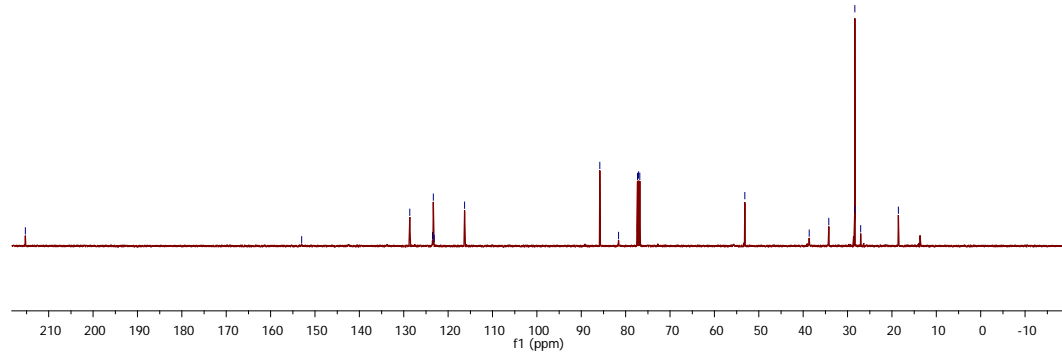

${ }^{13} \mathrm{C}$ NMR (1219 MHz, $\mathrm{CDCl}_{3}$ ) of compound (21) 


\section{Display Report}
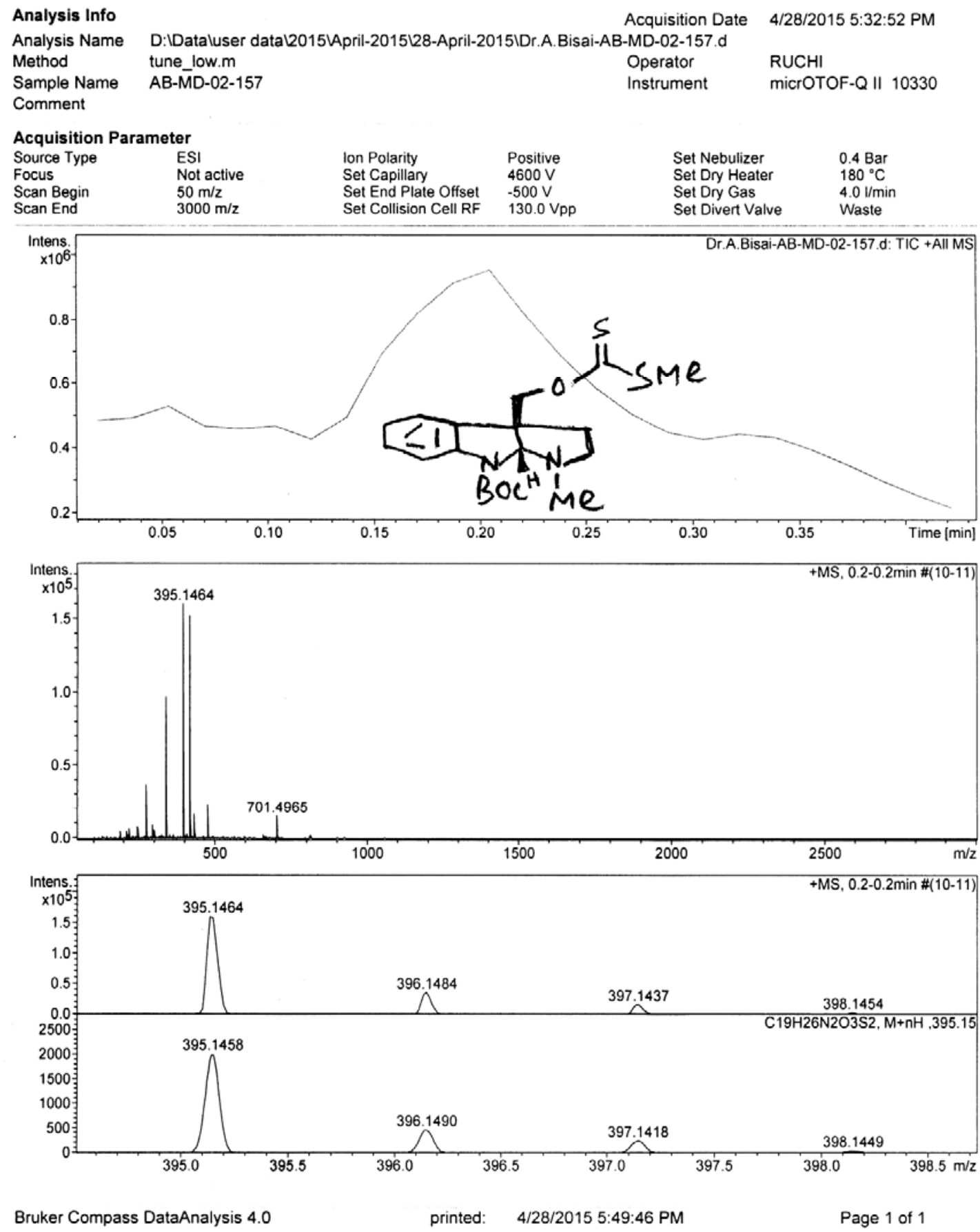

Scanned copy of mass spectrum of (21) 


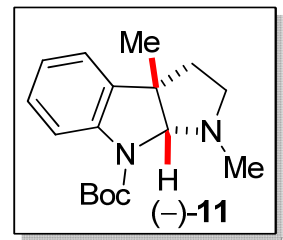

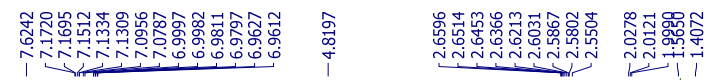

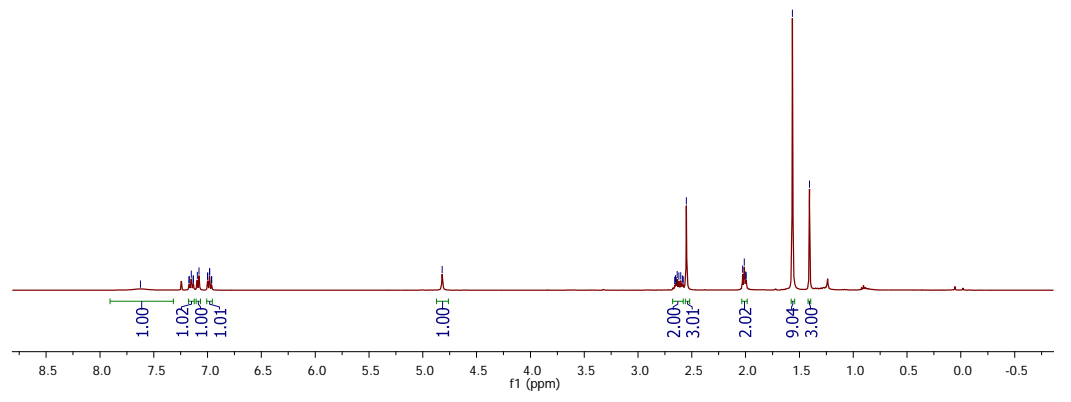

${ }^{1} \mathrm{H}$ NMR (400 MHz, $\mathrm{CDCl}_{3}$ ) of compound (11)
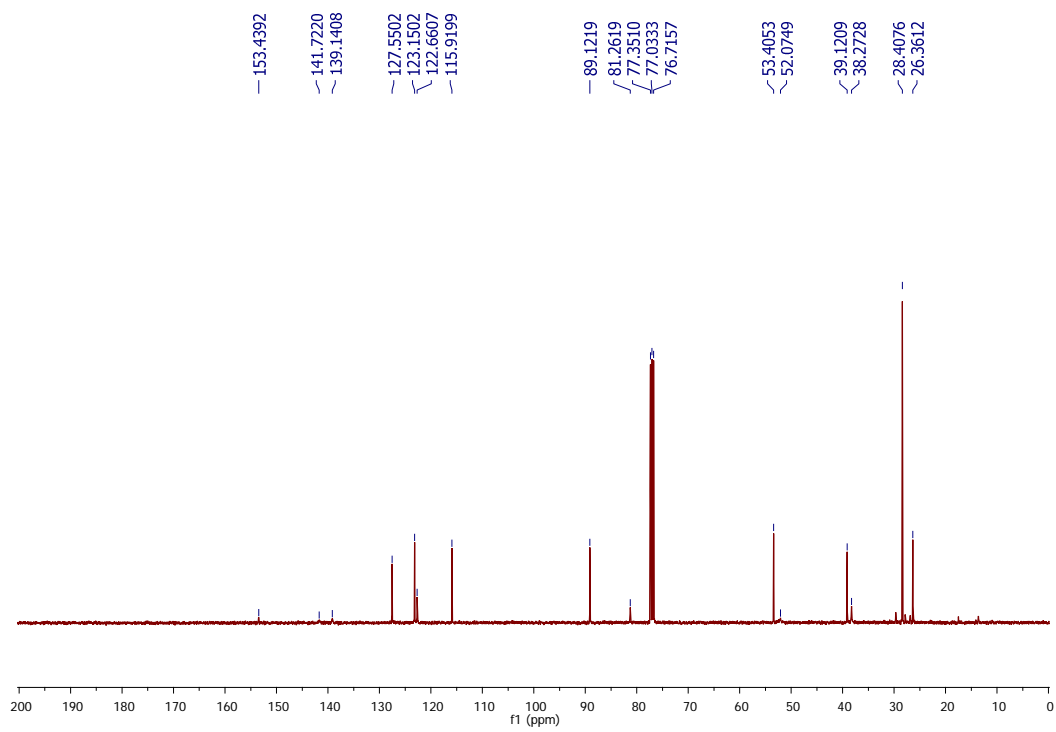

${ }^{13} \mathrm{C}$ NMR $\left(100 \mathrm{MHz}, \mathrm{CDCl}_{3}\right)$ of compound (11) 


\section{Display Report}
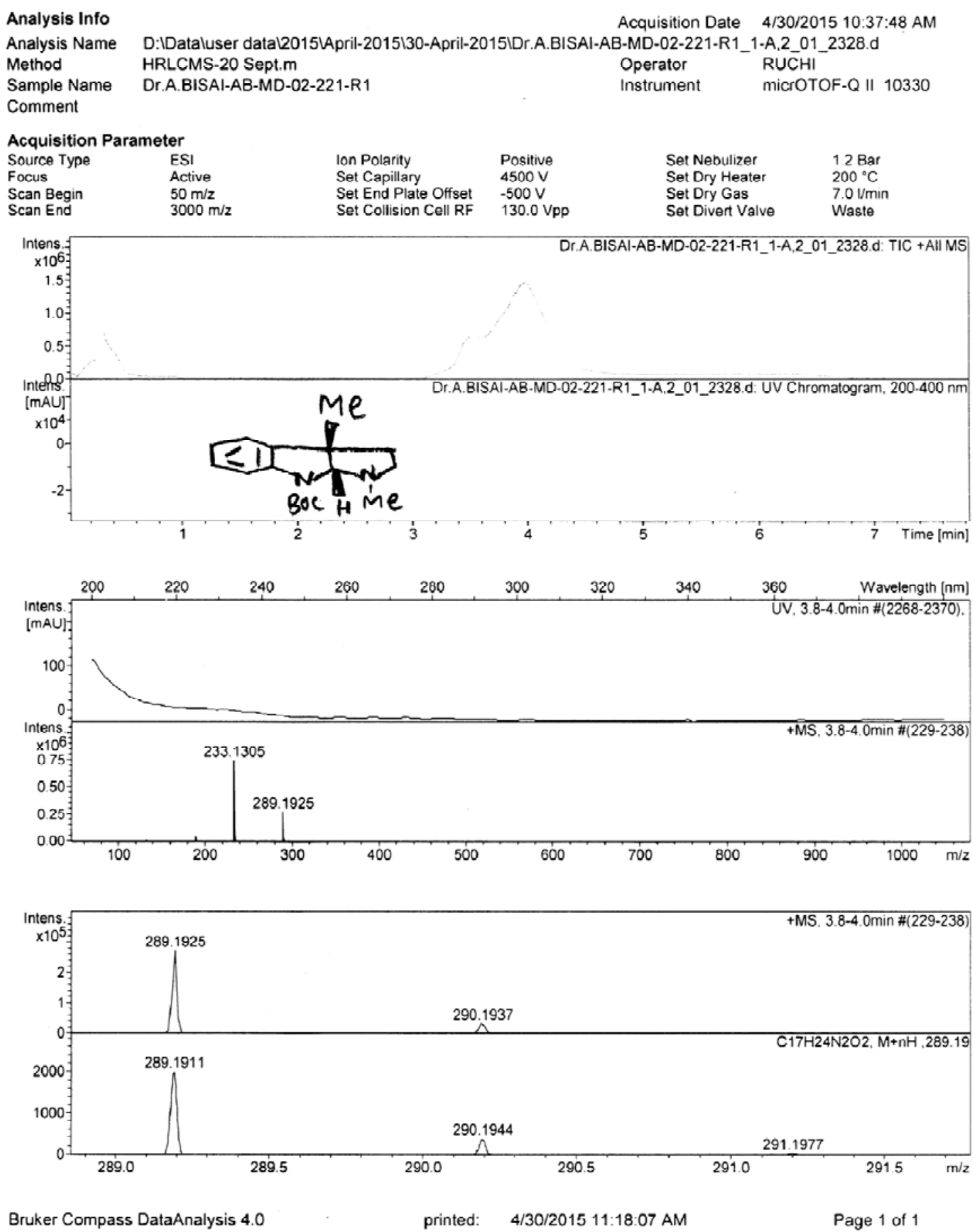

Scanned copy of mass spectrum of (11) 

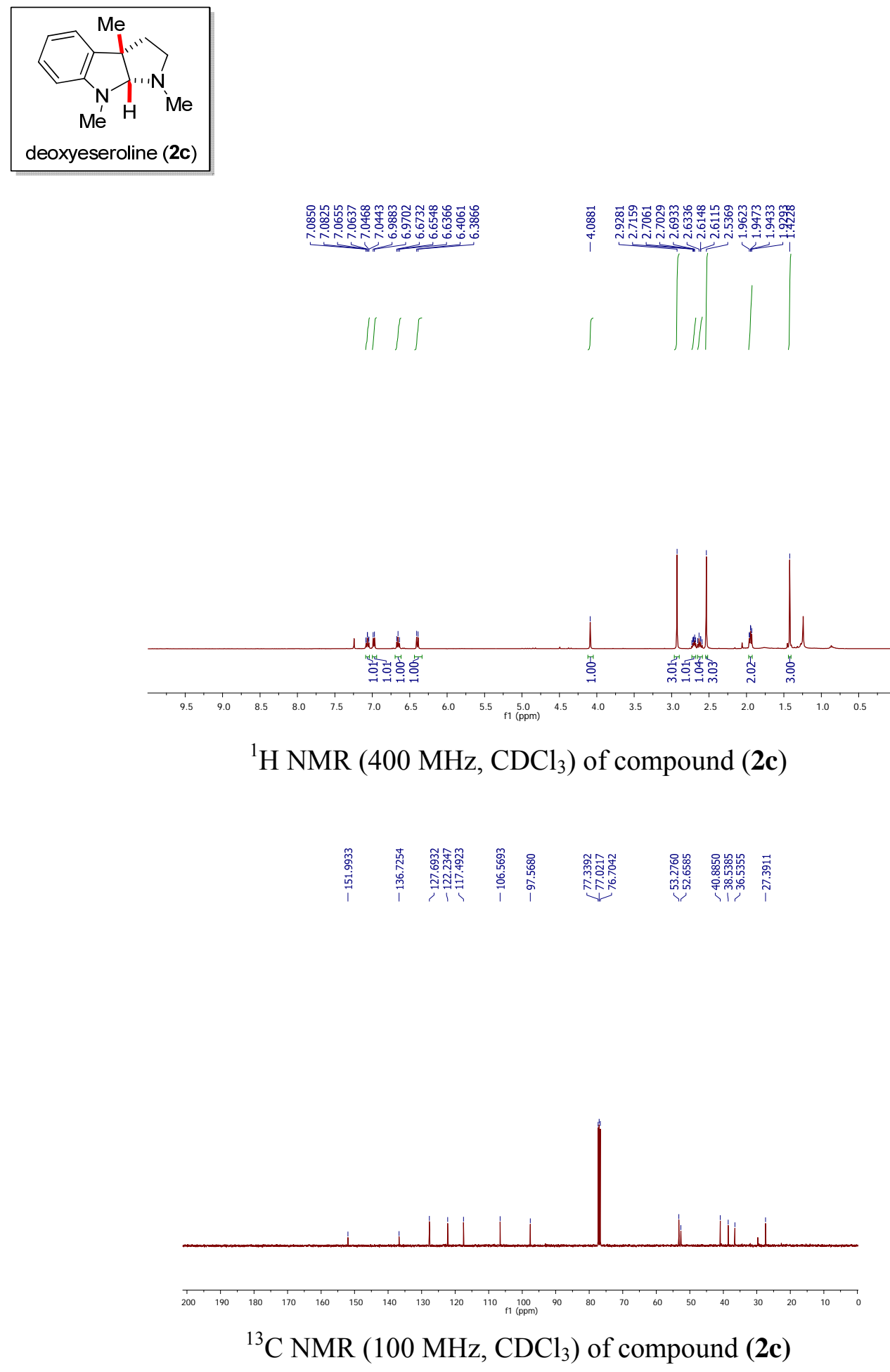


\section{Display Report}

Analysis Info Analysis Name

Method

Sample Name

Comment
Acquisition Date $\quad$ 5/7/2015 1:06:42 PM

D:IDataluser datal2015IMAY-2015107-MAY-2015IDr.A..Bisai-AB-MD-02-232 1-B,4 $012402 . d$

HRLCMS-20 Sept.m

Dr.A..Bisai-AB-MD-02-232
Operator $\quad \overline{\mathrm{RUCH}}$

Instrument micrOTOF-Q I| 10330
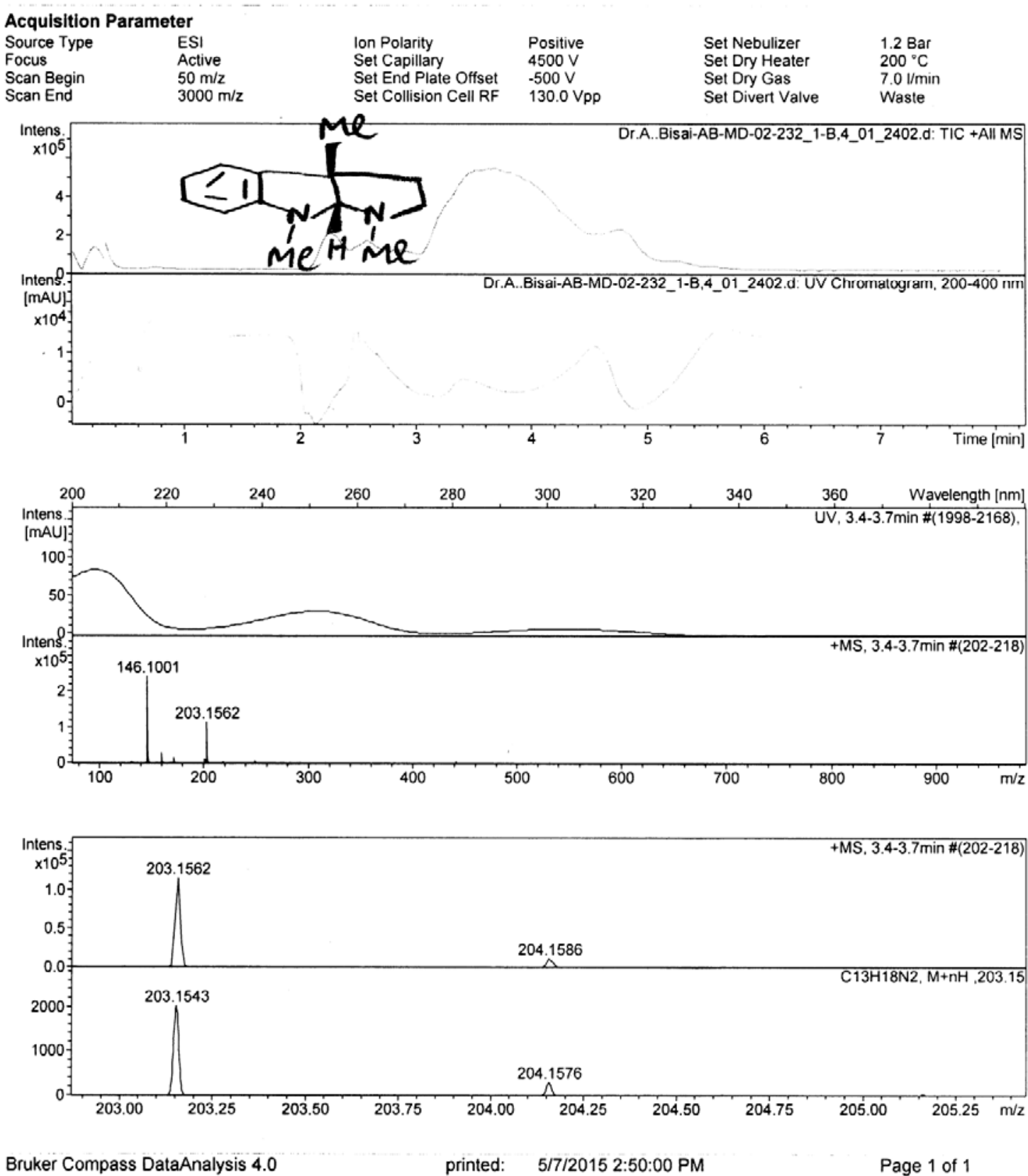

Scanned copy of mass spectrum of $(2 \mathbf{c})$ 


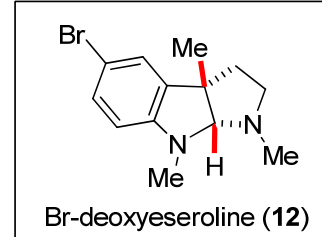

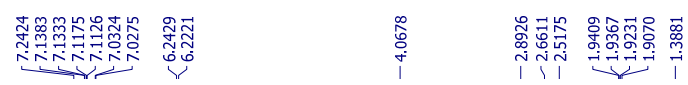
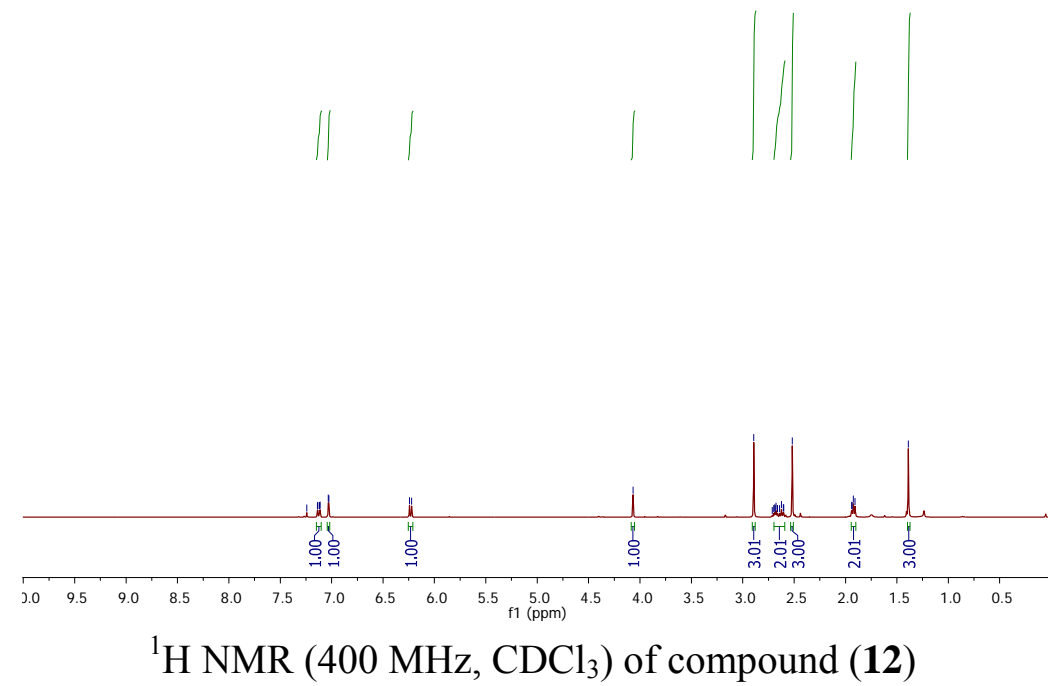

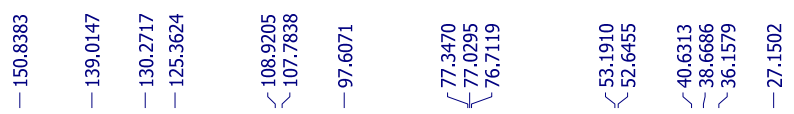

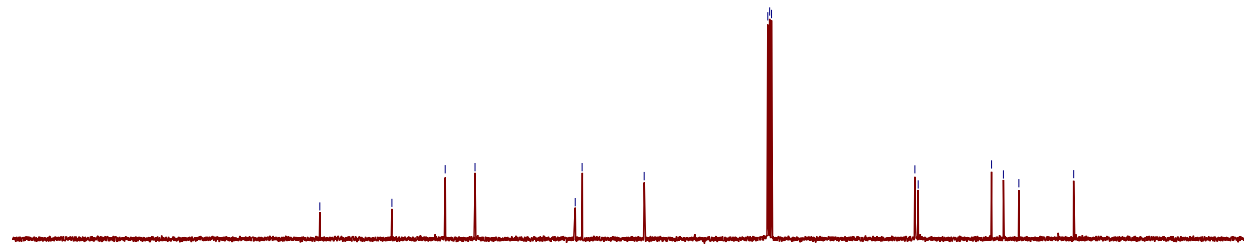

$\begin{array}{llllllllllllllllllllllll}200 & 190 & 180 & 170 & 160 & 150 & 140 & 130 & 120 & 110 & 100 & 90 & 80 & 70 & 60 & 50 & 40 & 30 & 20 & 10 & \mathrm{C}\end{array}$

${ }^{13} \mathrm{C}$ NMR (100 MHz, $\mathrm{CDCl}_{3}$ ) of compound (12) 


\section{Display Report}
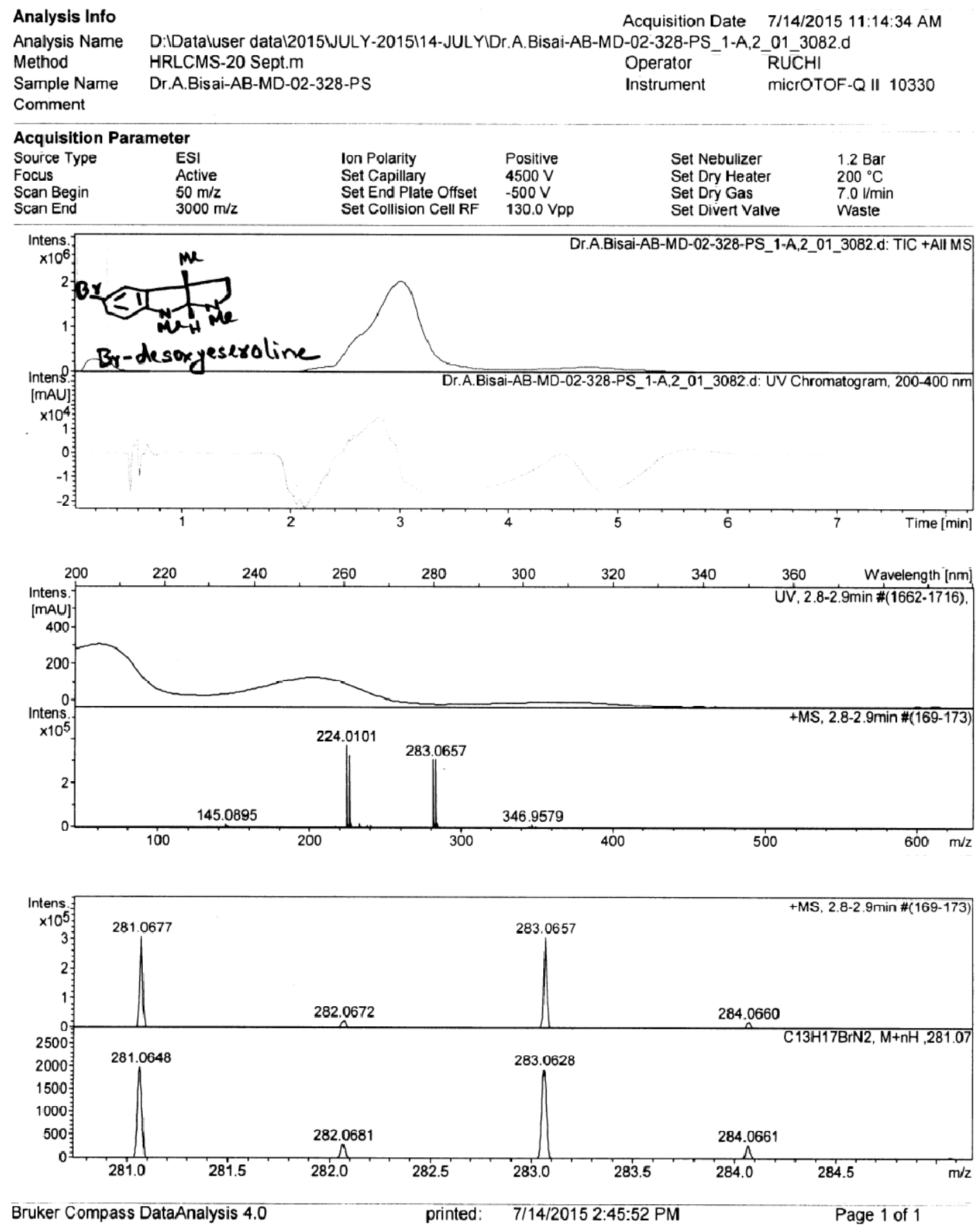

Scanned copy of mass spectrum of (12) 


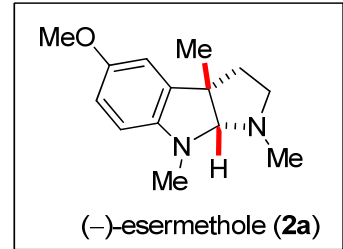

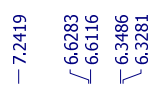

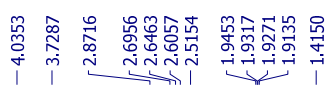
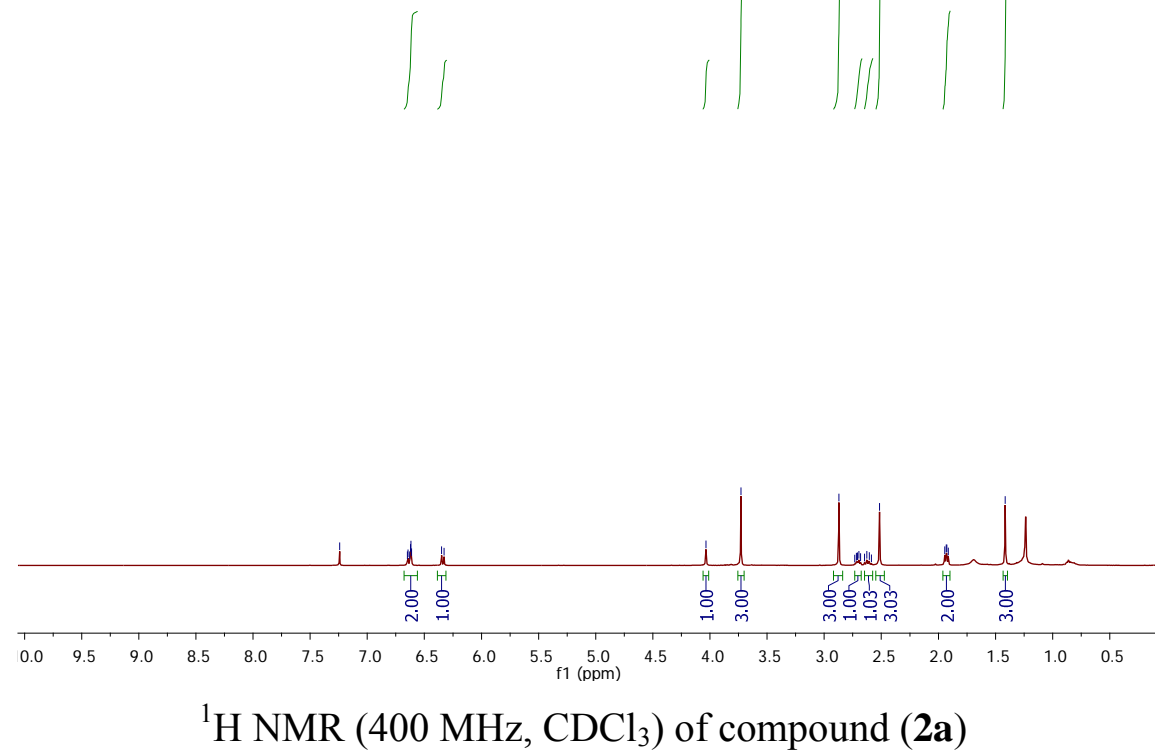

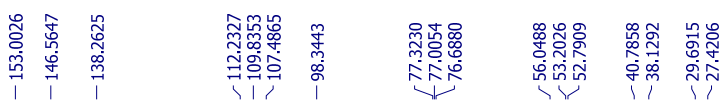

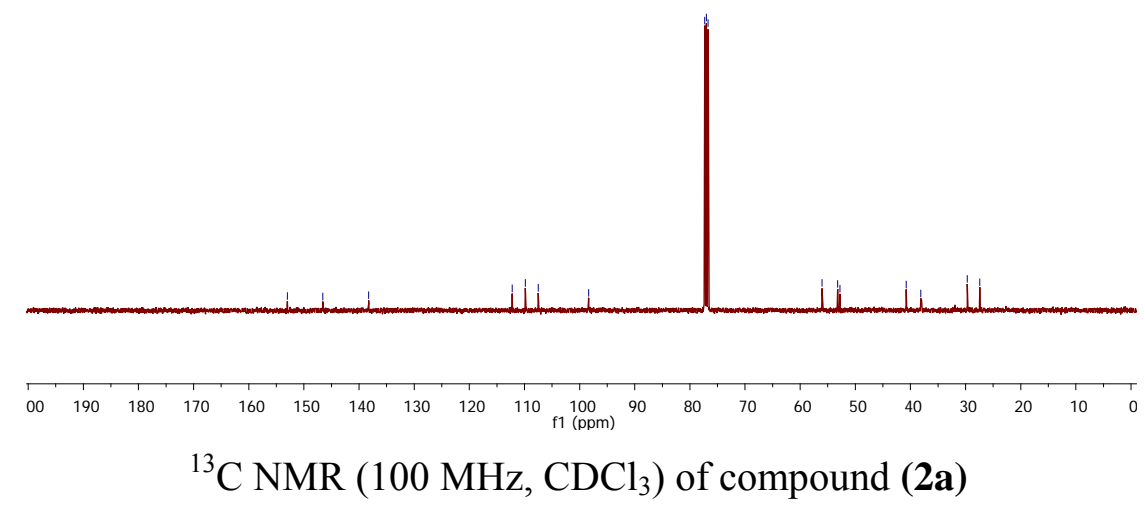




\section{Display Report}
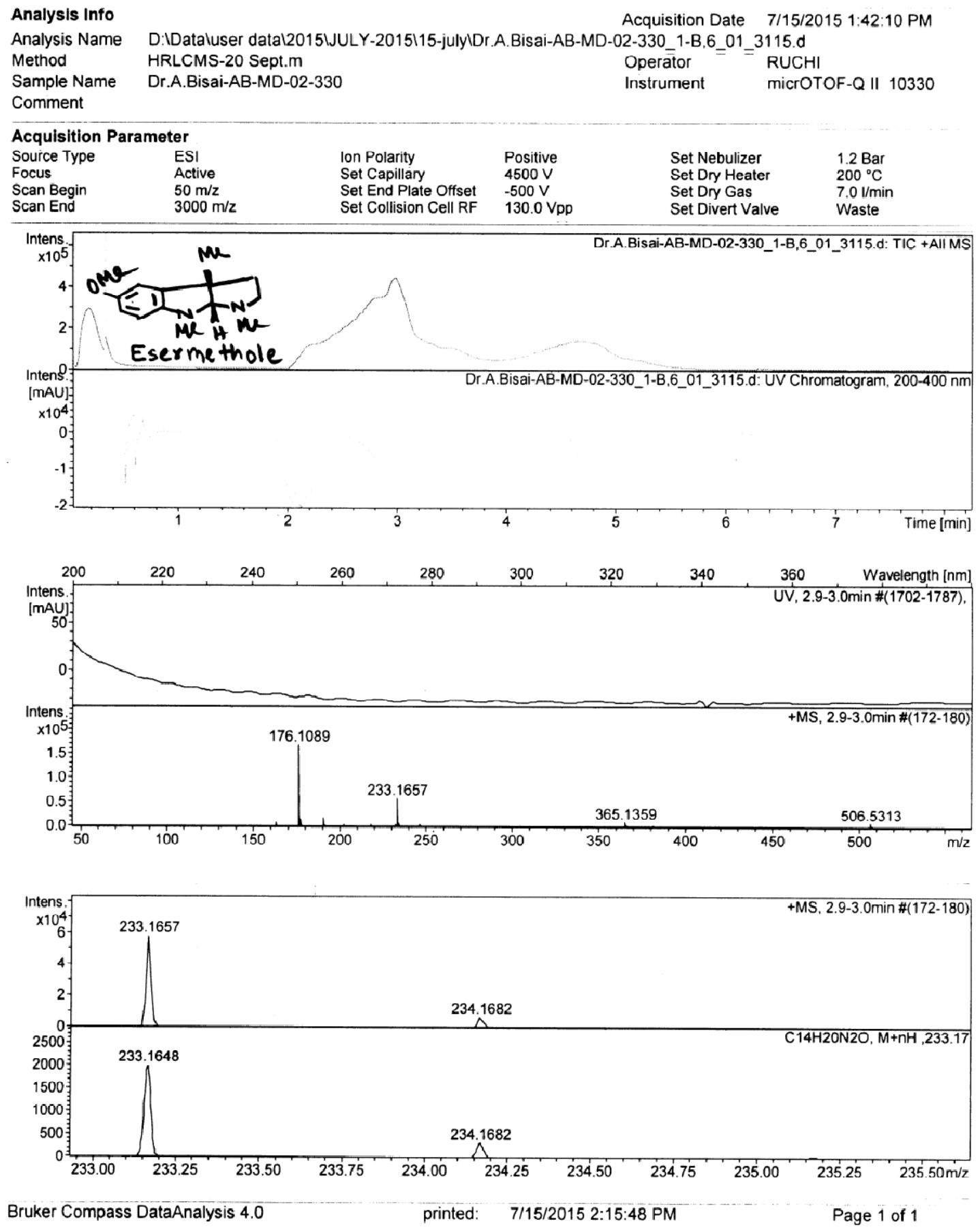

Scanned copy of mass spectrum of (2a) 


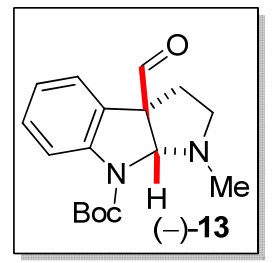

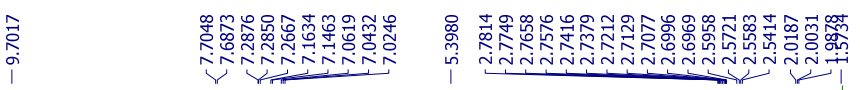
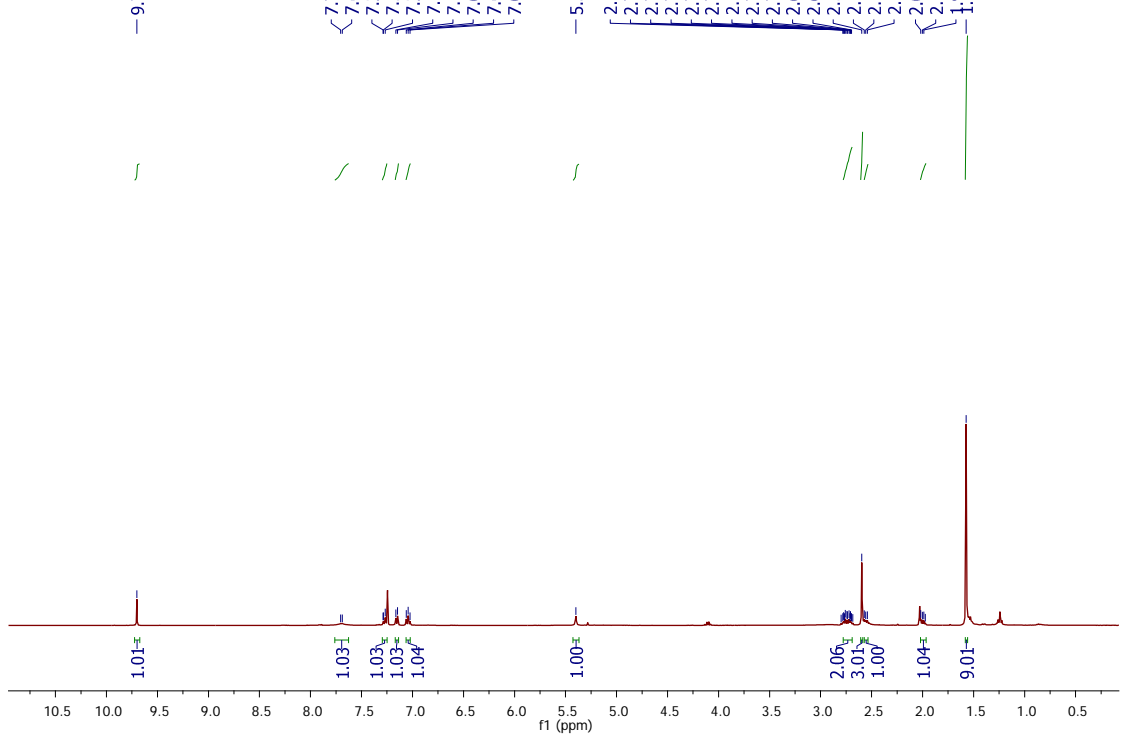

${ }^{1} \mathrm{H}$ NMR (400 MHz, $\mathrm{CDCl}_{3}$ ) of compound (13)

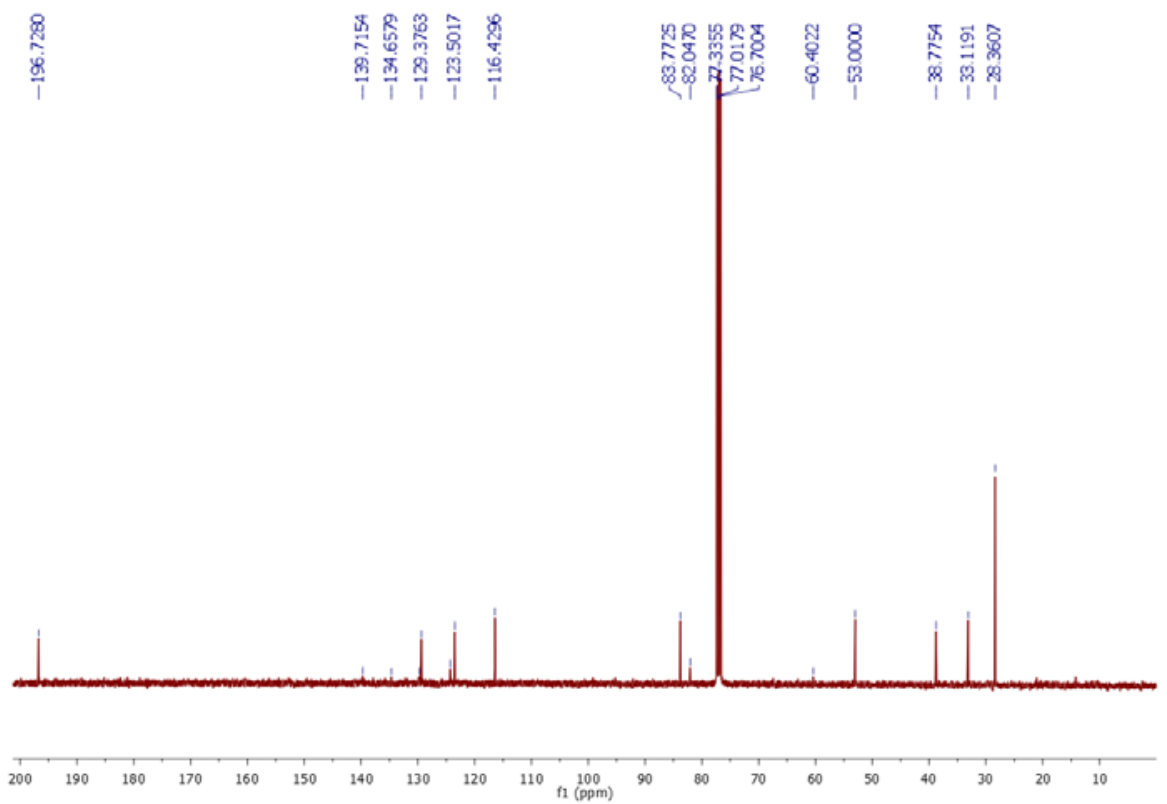

${ }^{13} \mathrm{C}$ NMR (100 MHz, $\mathrm{CDCl}_{3}$ ) of compound (13) 
Display Report

Analysis Info

Analysis Name

Method

Sample Name

Comment

Acquisition Parameter
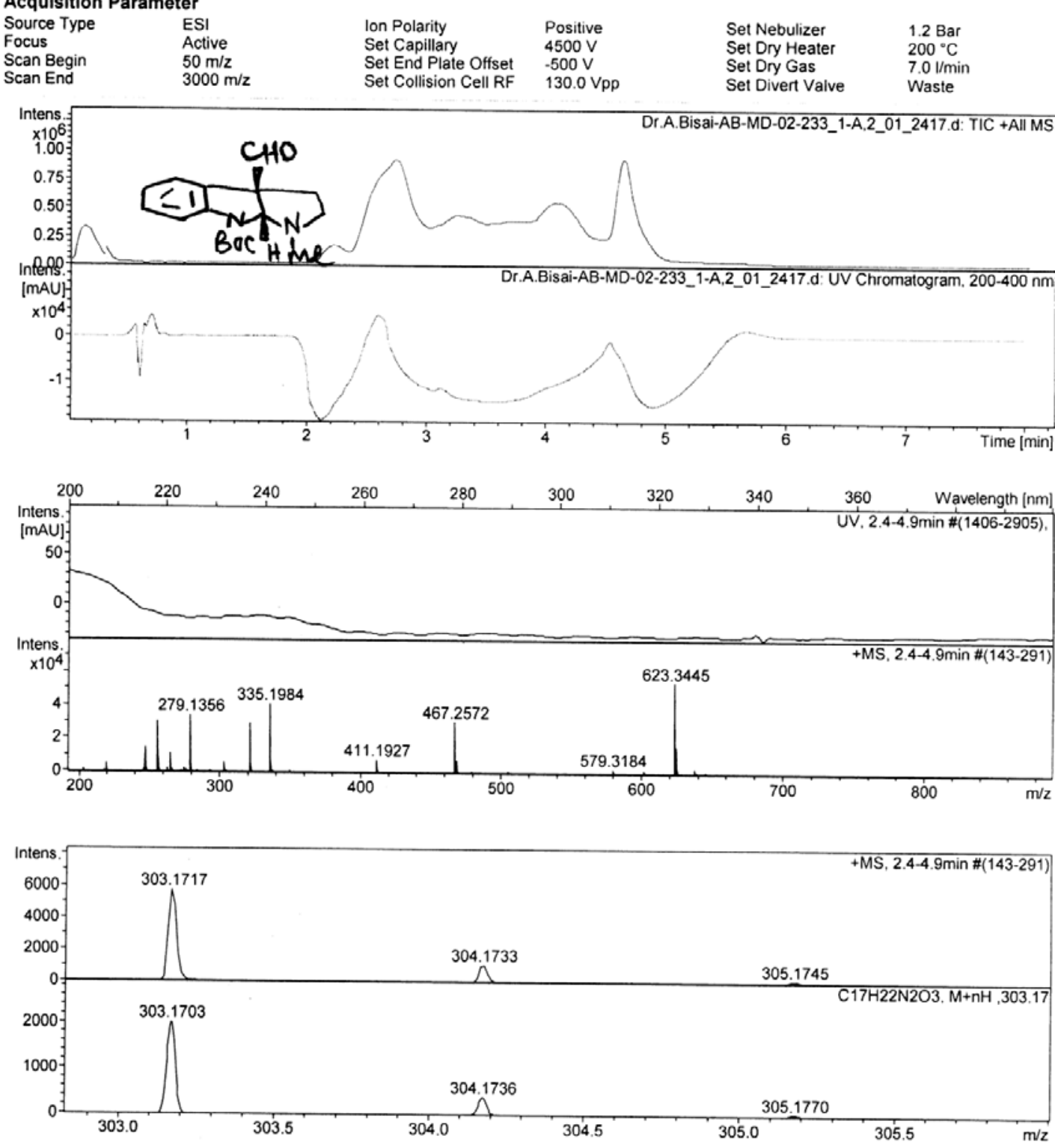

Bruker Compass DataAnalysis 4.0

printed: $\quad$ 5/8/2015 3:23:36 PM

Page 1 of 1

Scanned copy of mass spectrum of (13) 


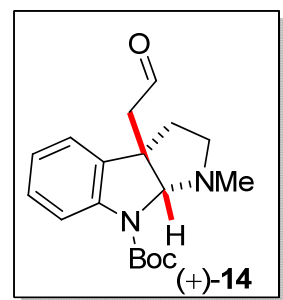

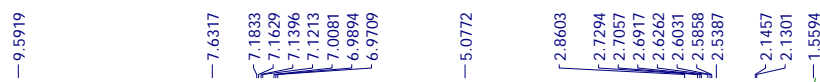

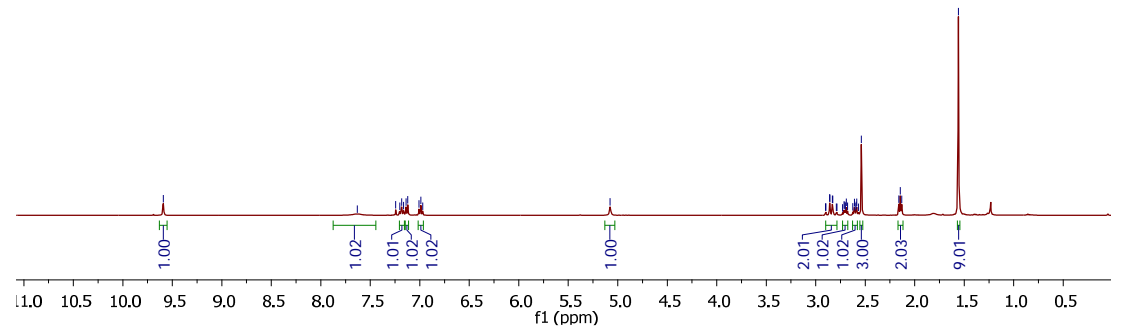

${ }^{1} \mathrm{H}$ NMR (400 MHz, $\mathrm{CDCl}_{3}$ ) of compound (14)

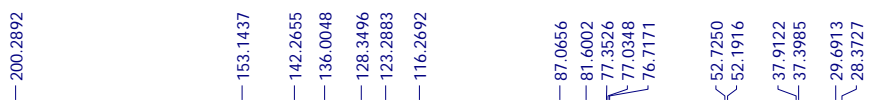

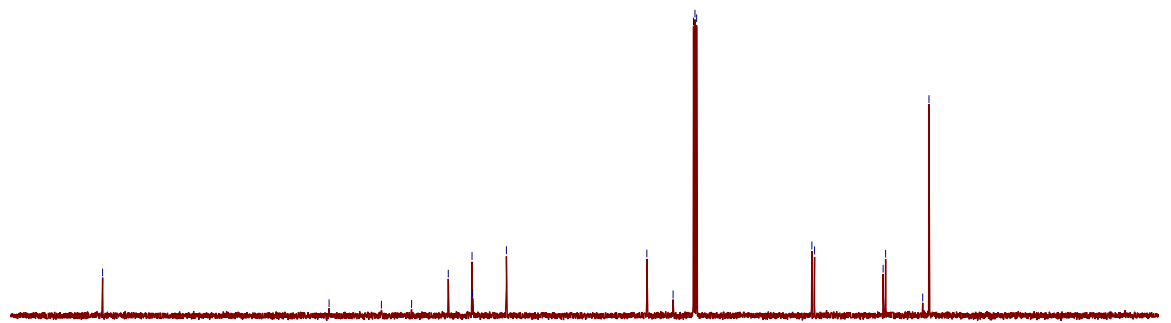

$\begin{array}{lllllllllllllllllllllllllllllllll}210 & 200 & 190 & 180 & 170 & 160 & 150 & 140 & 130 & 120 & 110 & 100 & 90 & 80 & 70 & 60 & 50 & 40 & 30 & 20 & 10 & 0 & -10\end{array}$

${ }^{13} \mathrm{C}$ NMR $\left(100 \mathrm{MHz}, \mathrm{CDCl}_{3}\right)$ of compound (14) 


\section{Display Report}
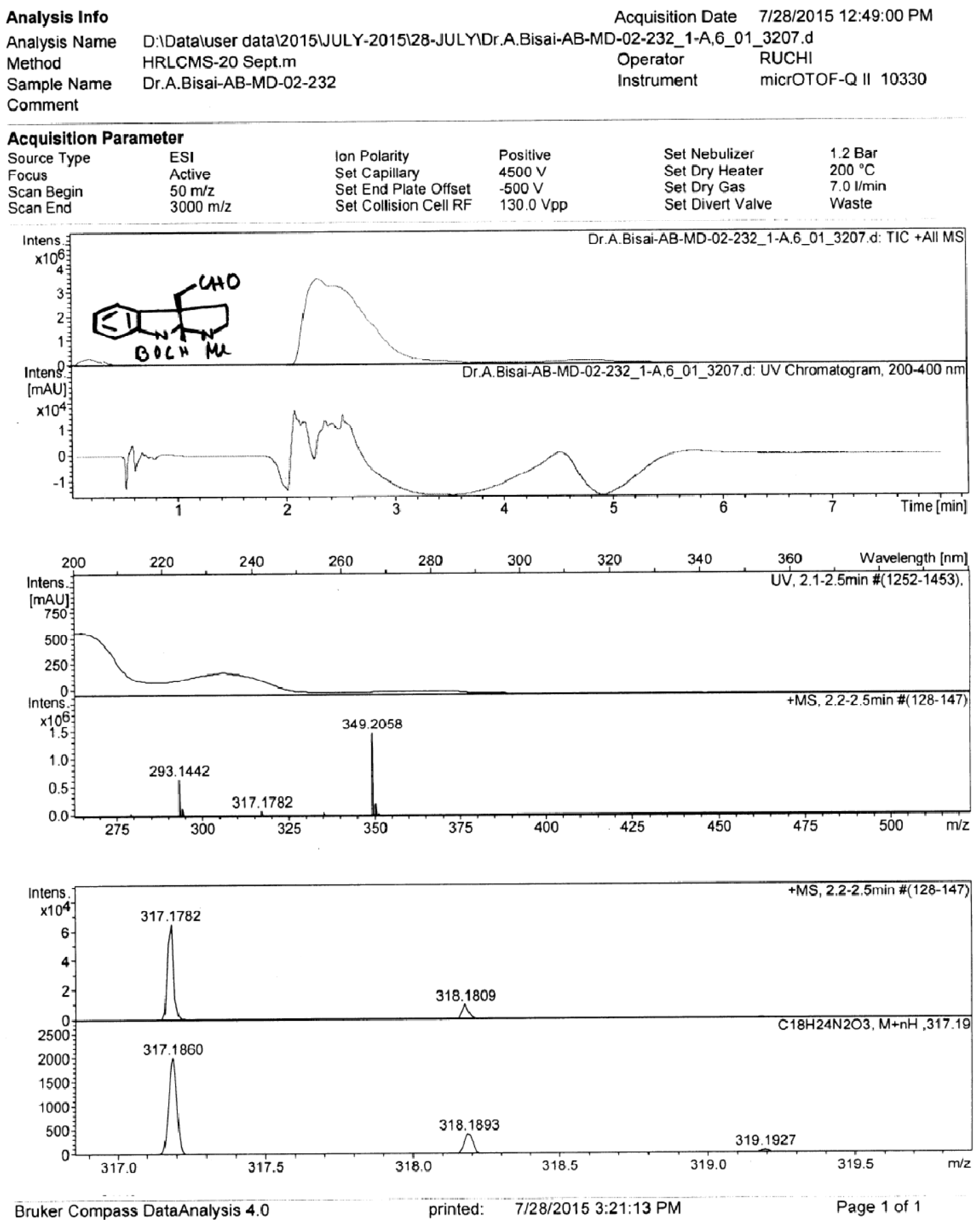

Scanned copy of mass spectrum of (14) 

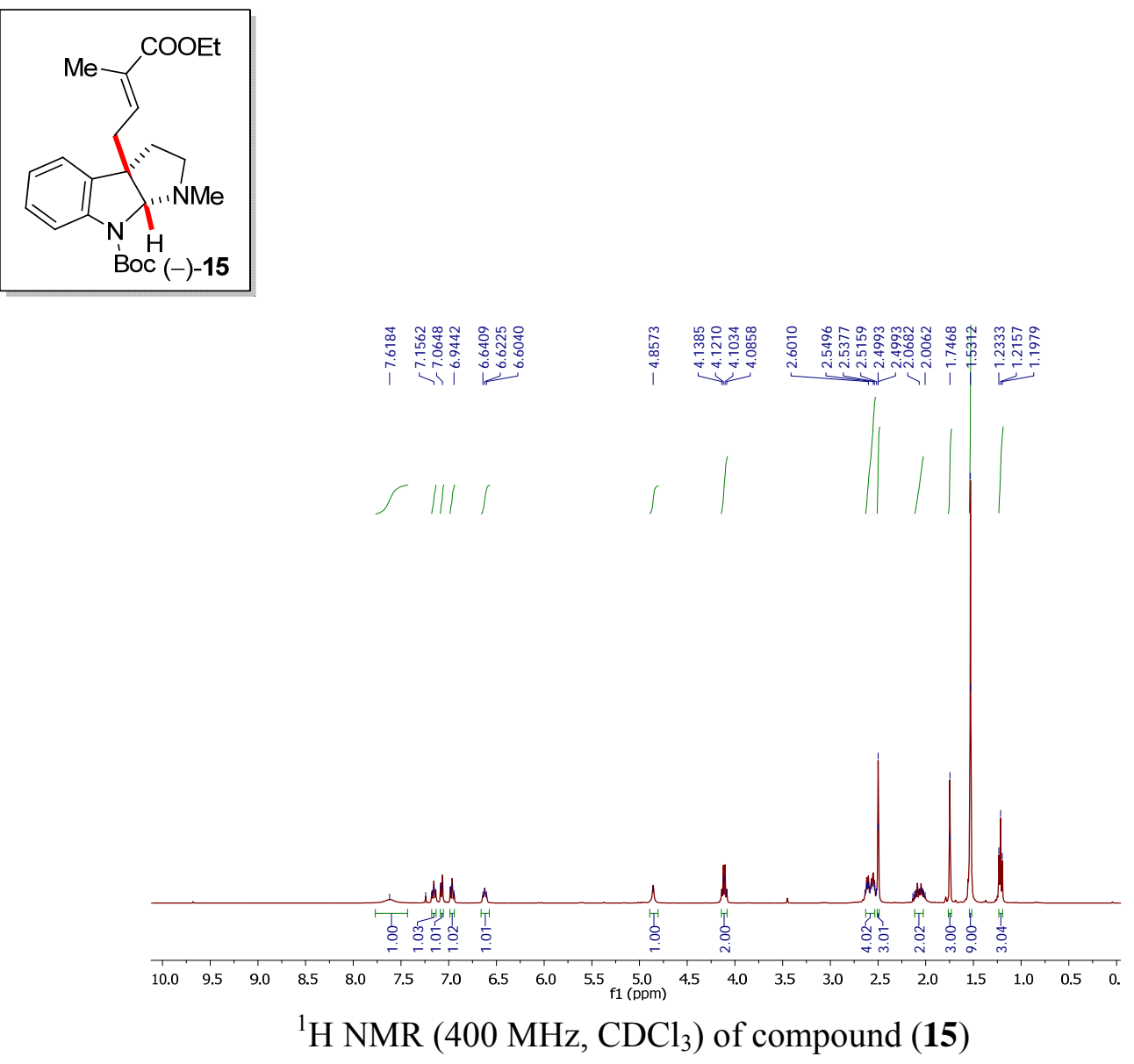

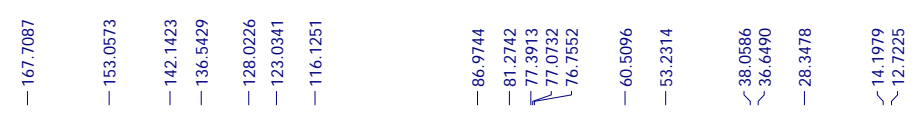

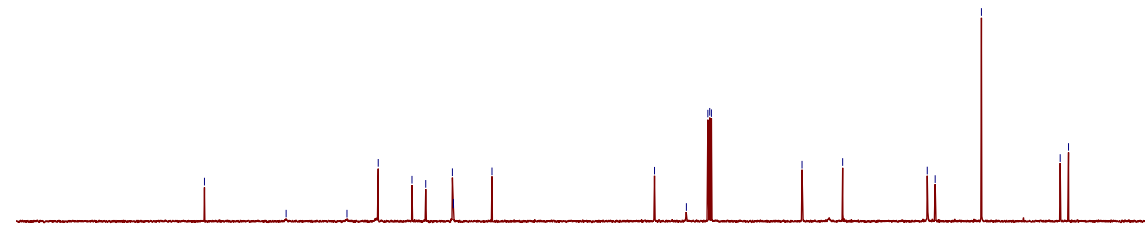

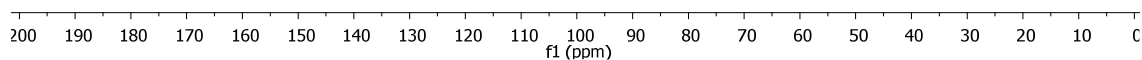

${ }^{13} \mathrm{C}$ NMR (100 MHz, $\mathrm{CDCl}_{3}$ ) of compound (15) 


\section{Display Report}
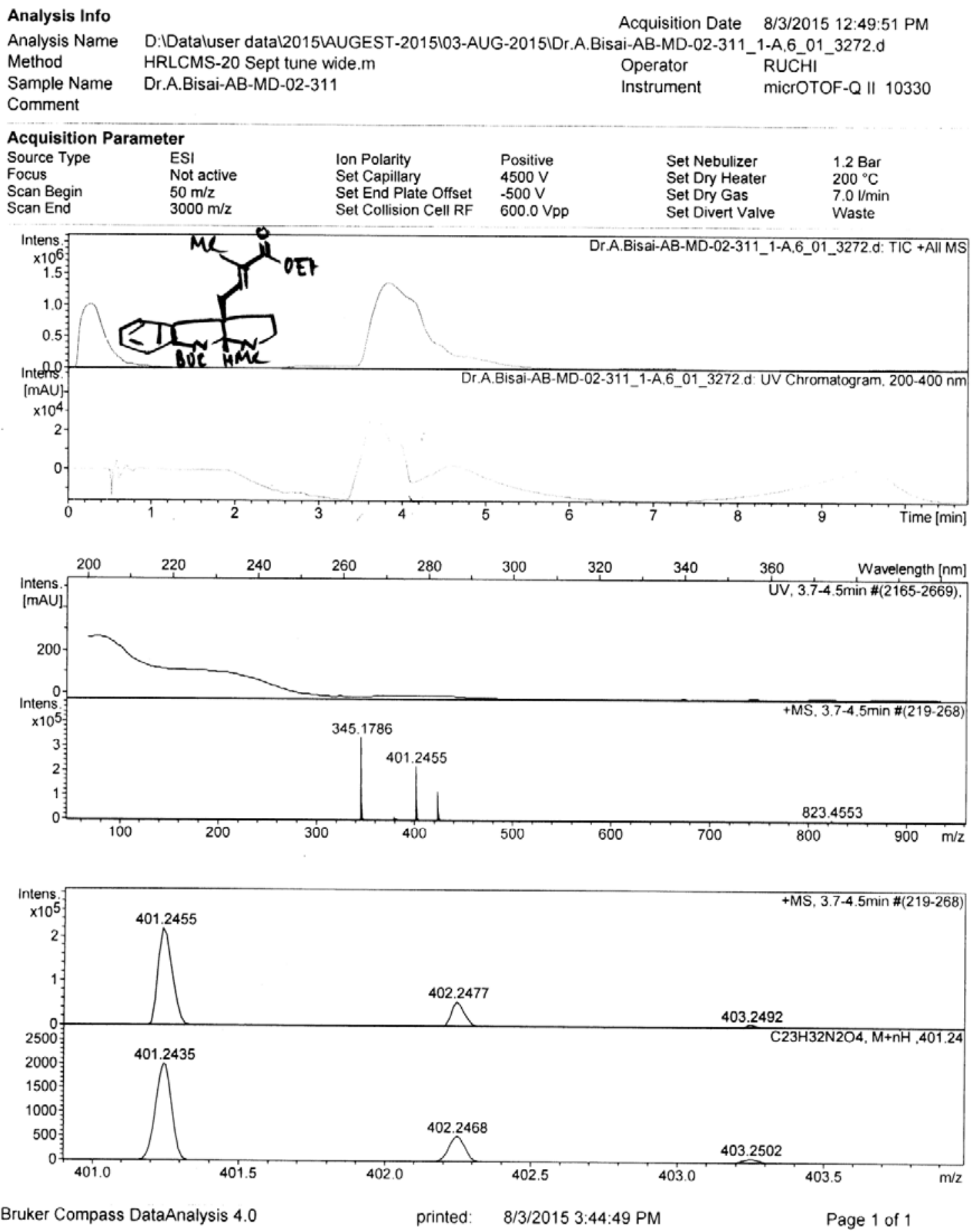

Scanned copy of mass spectrum of (15) 


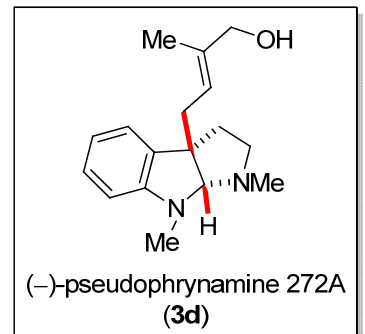

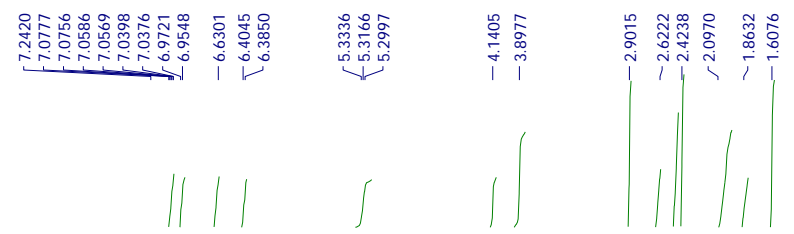

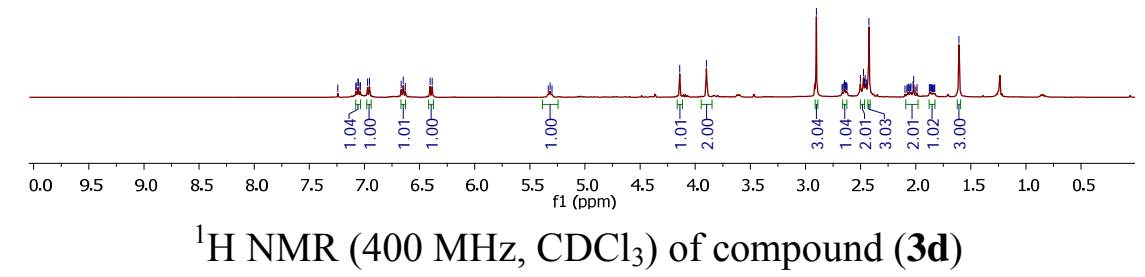

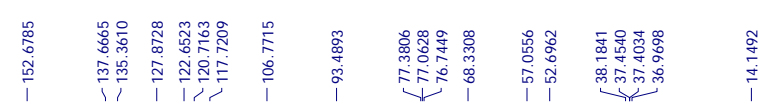

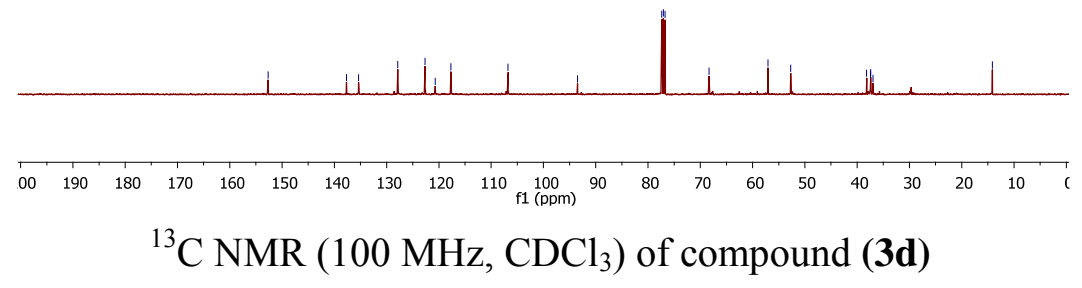


Display Report
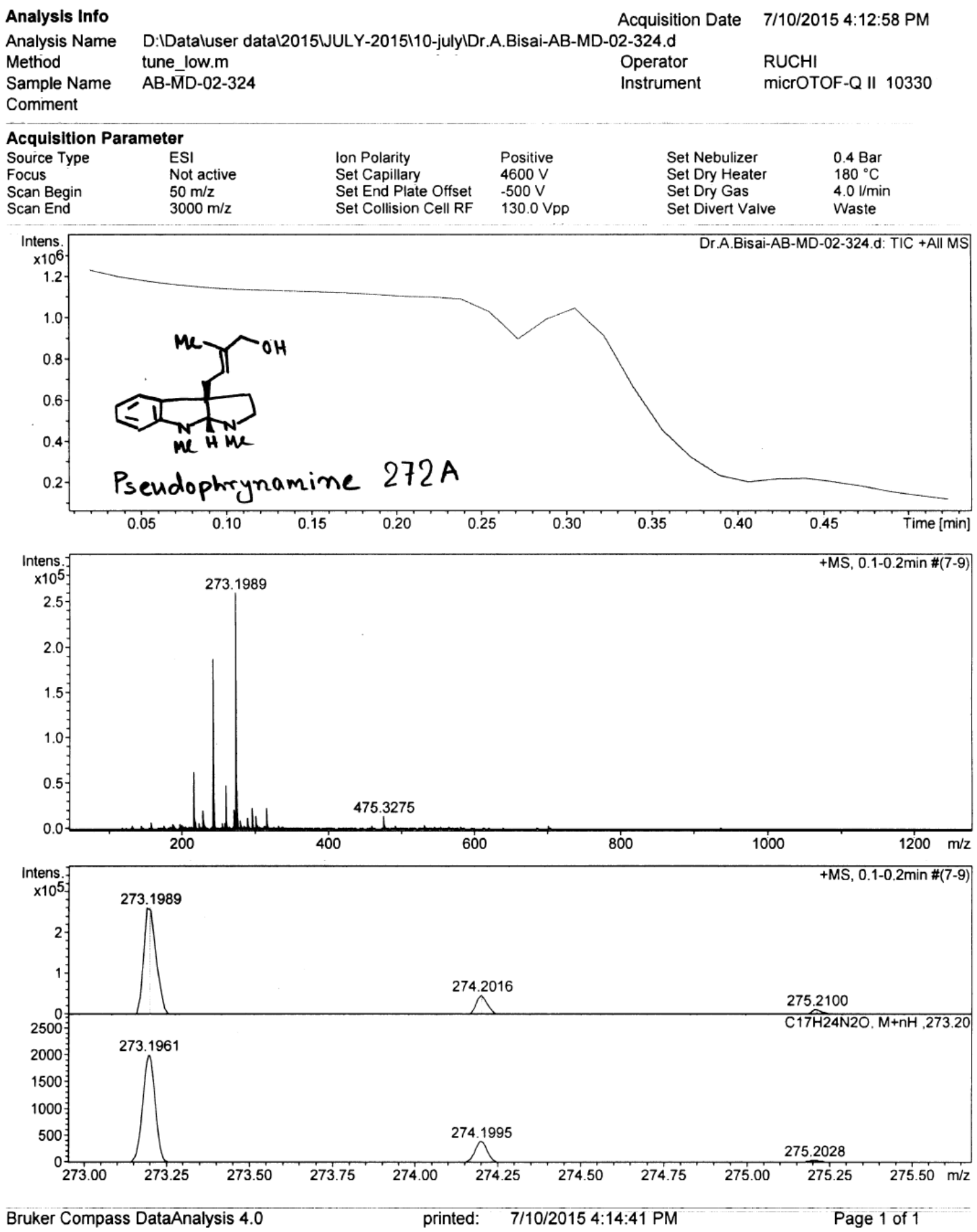

Scanned copy of mass spectrum of (3d) 

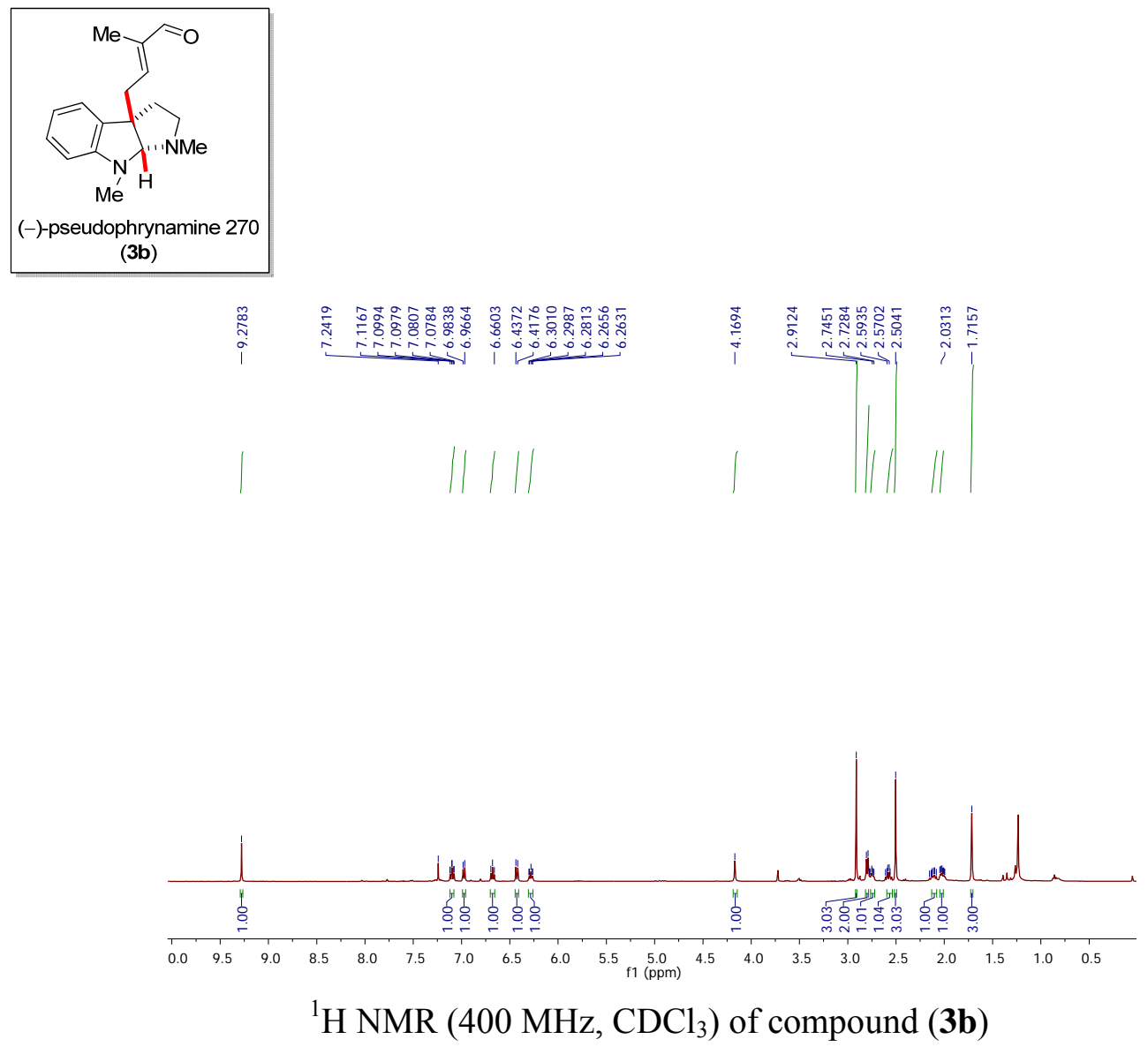

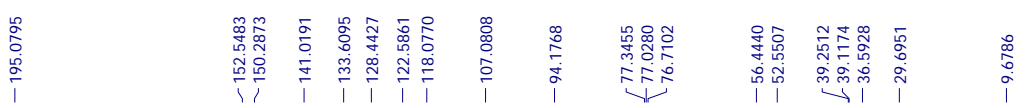

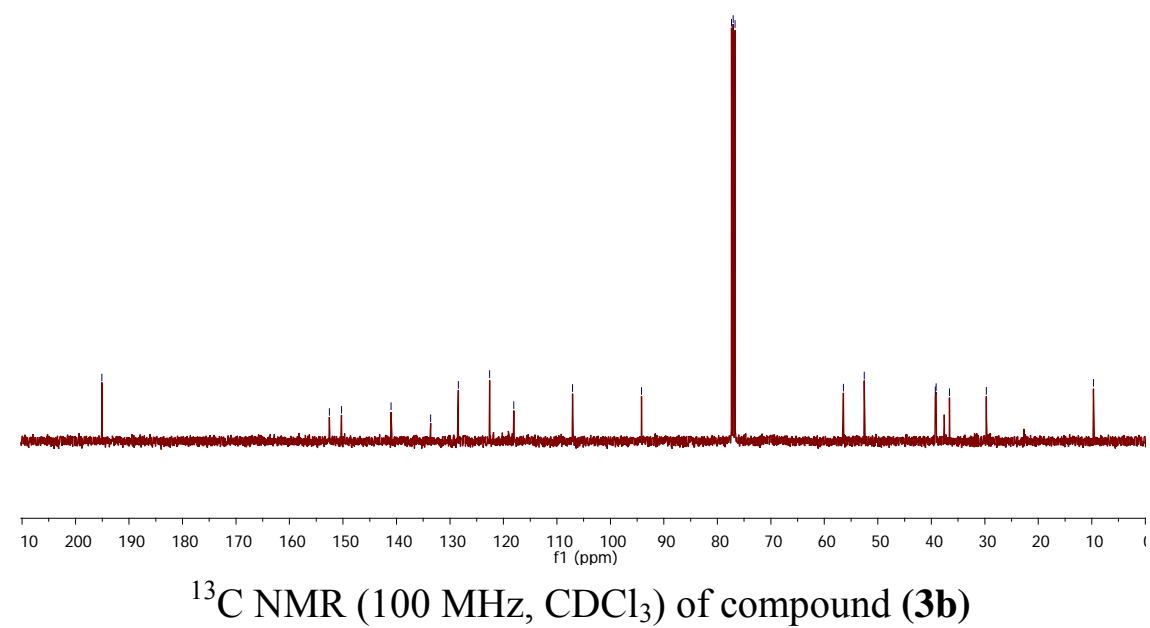




\section{Display Report}

Analysis Info

Analysis Name

Method

D:IDataluser datal2015WULY-2015121-JULYIDr.A.Bisai-AB-MD-02-340_1-B,4_01_3163.d
HRLCMS-20 Sept.m

Sample Name

Dr.A.Bisai-AB-MD-02-340

Instrument

micrOTOF-Q ॥ 10330

\section{Acquisition Parameter}

\begin{tabular}{llllll} 
Acquisition Parameter & & & \\
Source Type & ESI & Ion Polarity & Positive & Set Nebulizer & 1.2 Bar \\
Focus & Active & Set Capillary & $4500 \mathrm{~V}$ & Set Dry Heater & $200^{\circ} \mathrm{C}$ \\
Scan Begin & $50 \mathrm{~m} / \mathbf{z}$ & Set End Plate Offset & $-500 \mathrm{~V}$ & Set Dry Gas & 7.0 Umin \\
Scan End & $3000 \mathrm{~m} / \mathrm{z}$ & Set Collision Cell RF & $130.0 \mathrm{Vpp}$ & Set Divert Valve & Waste \\
\hline
\end{tabular}

Set Collision Cell RF

Dr.A.Bisai-AB-MD-02-340_1-B,4_01_3163.d: TIC +All MS
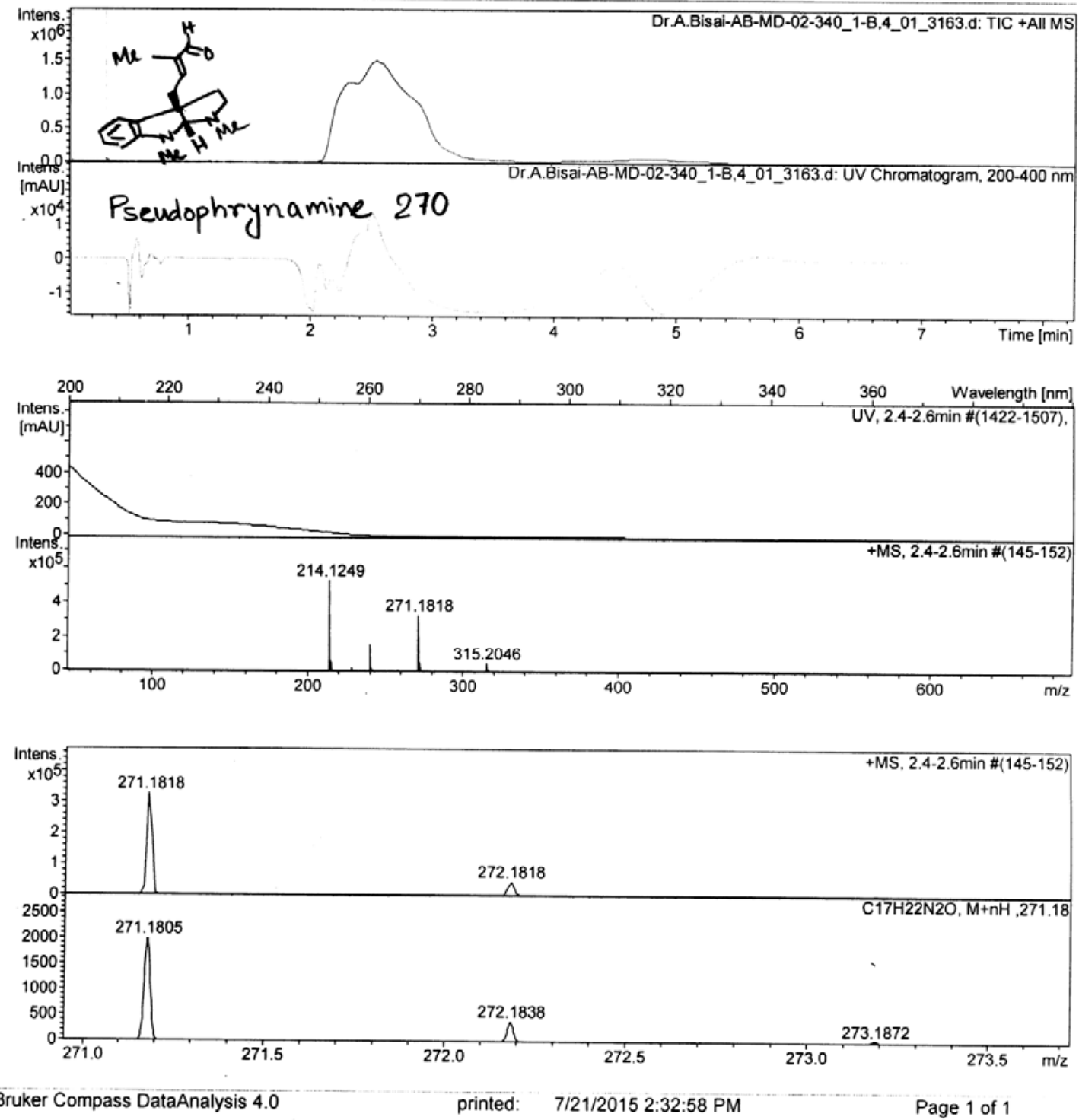

Scanned copy of mass spectrum of (3b) 